\title{
Finite-temperature dynamics of low-dimensional quantum systems with DMRG methods
}

\author{
Dissertation \\ zur Erlangung des mathematisch-naturwissenschaftlichen \\ Doktorgrades \\ "Doctor rerum naturalium" \\ der Georg-August-Universität Göttingen \\ im Promotionsprogramm ProPhys \\ der Georg-August University School of Science (GAUSS)
}

vorgelegt von

Alexander Clemens Tiegel

aus Hoya/Weser

Göttingen, 2016 


\section{Betreuungsausschuss}

Prof. Dr. Thomas Pruschke

Institut für Theoretische Physik

Georg-August-Universität Göttingen

Prof. Dr. Andreas Honecker

Laboratoire de Physique Théorique et Modélisation

Université de Cergy-Pontoise

\section{Mitglieder der Prüfungskommission}

Referent: Prof. Dr. Stefan Kehrein

Institut für Theoretische Physik

Georg-August-Universität Göttingen

Korreferent: $\quad$ Prof. Dr. Andreas Honecker

Laboratoire de Physique Théorique et Modélisation

Université de Cergy-Pontoise

Zweiter Korreferent: Prof. Dr. Kai P. Schmidt (kein Mitglied der Prüfungskommission) Institut für Theoretische Physik I

Friedrich-Alexander-Universität Erlangen-Nürnberg

\section{Weitere Mitglieder der Prüfungskommission}

PD Dr. Salvatore R. Manmana

Institut für Theoretische Physik

Georg-August-Universität Göttingen

Prof. Dr. Stefan Mathias

I. Physikalisches Institut

Georg-August-Universität Göttingen

Prof. Dr. Reiner Kree

Institut für Theoretische Physik

Georg-August-Universität Göttingen

Prof. Dr. Annette Zippelius

Institut für Theoretische Physik

Georg-August-Universität Göttingen

Tag der mündlichen Prüfung: 25.07.2016 


\section{Abstract}

This thesis is concerned with the numerical study of one-dimensional (1D) spin- $1 / 2$ quantum magnets and related method development. Its focus is on the calculation of dynamical spin correlation functions both at zero and finite temperature. This is motivated by the accessibility of dynamical quantities in experiments such as inelastic neutron scattering (INS) and electron spin resonance (ESR). The numerical methods used in this thesis are based on extensions of the density-matrix renormalization group (DMRG) and are formulated in the framework of matrix product states (MPS). While zero-temperature dynamical correlation functions are computed with existing MPS frequency-domain methods, an MPS frequency-domain approach for their calculation at finite temperature is developed in this thesis. The new method combines the Liouville-space formulation of the dynamics with a moment expansion of the dynamical correlation function. The majority of the results are obtained via MPS-based Chebyshev expansions.

These numerical techniques are applied to two different model systems describing real materials. The first one is the material copper pyrimidine dinitrate $(\mathrm{Cu}-\mathrm{PM})$ which is modeled by a 1D spin-1/2 Heisenberg antiferromagnet with Dzyaloshinskii-Moriya interactions. The spin dynamics of this model is studied in an applied magnetic field and compared to ESR experiments. Zero-temperature calculations for momentum- and frequency-resolved dynamical quantities give direct access to the intensity of the elementary excitations and go beyond the low-energy description by the quantum sine-Gordon model. Thus, a deviation from the Lorentz invariant dispersion for the single-soliton resonance is found. The presence of the strongest boundary bound state previously predicted from a boundary sine-Gordon field theory is confirmed, while composite boundary-bulk excitations have too low intensities to be found in the numerical results. At finite temperature, there is a temperature-induced crossover of the soliton. Moreover, additional temperature effects such as interbreather transitions emerge, which is confirmed by accompanying ESR experiments on $\mathrm{Cu}-\mathrm{PM}$ over a wide range of the applied field strength.

The second system studied in this thesis is the compound $\mathrm{BaCu}_{2} \mathrm{~V}_{2} \mathrm{O}_{8}$. It is shown that the magnetic properties of this quasi-1D material can be described by a strongly alternating antiferromagnetic-ferromagnetic spin-1/2 Heisenberg chain. As found for other dimerized systems, the strong correlations in $\mathrm{BaCu}_{2} \mathrm{~V}_{2} \mathrm{O}_{8}$ persist even at elevated temperatures. Moreover, these correlations lead to an asymmetric lineshape broadening of the magnetic excitations at finite temperature. Upon raising the temperature, an increasingly asymmetric lineshape is observed in high-resolution inelastic neutron scattering (INS) experiments for $\mathrm{BaCu}_{2} \mathrm{~V}_{2} \mathrm{O}_{8}$. In this thesis, the lineshape is calculated by the MPS finite-temperature method developed as a part of this work. Comparing these results to the INS data and the lineshape obtained by a diagrammatic approach, excellent agreement is found over a broad temperature range. This demonstrates that coherent quantum behavior persists at elevated temperatures in $\mathrm{BaCu}_{2} \mathrm{~V}_{2} \mathrm{O}_{8}$ and that it can be predicted quantitatively. 



\section{Danksagung/Acknowledgments}

Ein ganz besonderer Dank gilt Thomas Pruschke (+2016) und Andreas Honecker, die mir ermöglicht haben, diese Arbeit anzufertigen und mich stets hervorragend betreut und unterstützt haben. Sie waren immer gute Ansprechpartner für Fragen und gaben wertvolle Hinweise. Dies blieb auch weiterhin so, als Thomas Pruschke im Jahr 2014 schwer erkrankte und Andreas Honecker wenig später eine Professorenstelle in CergyPontoise bei Paris antrat. Ich bin beiden für ihr Engagement sehr dankbar.

Außerdem möchte ich mich bei Salvatore Manmana bedanken, der mir ebenfalls als wichtiger Betreuer mit Rat und Tat zur Seite stand. Dafür ein herzliches „grazie mille“.

Überdies danke ich Stefan Kehrein dafür, dass er das Referat für diese Arbeit übernommen hat. Außerdem bin ich ihm für Gespräche über fachliche und organisatorische Aspekte dankbar. Ein Dankeschön gilt ebenfalls Kai P. Schmidt für die Anfertigung des externen Gutachtens sowie den Mitgliedern der Prüfungskommission.

Des Weiteren bedanke ich mich insbesondere bei Benjamin Lenz, Kurt Schönhammer, Benedikt Fauseweh, Robert Schade und Salvatore Manmana für die Unterstützung beim Korrekturlesen meiner Dissertation. Außerdem bin ich meinem Bürokollegen Thomas Köhler für etliche Diskussionen sowie nützliche Programmier-Tipps dankbar.

Mir hat das angenehme Arbeitsklima und die gute Atmosphäre in der Arbeitsgruppe für Festkörpertheorie sehr gefallen. Vielen Dank für die gemeinsam verbrachte Zeit und all die Diskussionen über Physik und auch andere Themen.

Mein Dank gilt auch der Helmholtz Gemeinschaft, die diese Arbeit über das Helmholtz Virtuelle Institut New states of matter and their excitations (VH-VI-521) förderte. Diese Form der Zusammenarbeit ermöglichte mir, auf mehreren Workshops den wissenschaftlichen Austausch mit nationalen und internationalen Kollegen. Ich bin dankbar für die daraus hervorragenden Kollaborationen im Rahmen dieser Arbeit.

In this respect, special thanks go to Ekaterina Klyushina and Bella Lake from the Helmholtz-Zentrum Berlin. Their high-resolution inelastic neutron scattering data are compared to the numerical results obtained in this thesis (Chapter 7). Another important contribution to this project has been made by Benedikt Fauseweh and Götz Uhrig from the TU Dortmund. I thank all of them for many discussions and a great collaboration.

Moreover, I am grateful for a further experimental collaboration with Alexey Ponomaryov, Sergei Zvyagin, and Ralf Feyerherm. Chapter 6 of this thesis largely benefited from the contributed ESR experiments conducted at the Dresden High Magnetic Field Laboratory as well as their comments.

Außerdem danke ich meiner Familie ganz herzlich für die uneingeschränkte Unterstützung. Dieser Dank richtet sich insbesondere an meine Ehefrau Marie Tiegel und meine Mutter. 


\section{List of publications}

Parts of this thesis have been published in peer-reviewed journals. The corresponding references are listed below.

[TMPH14] A. C. Tiegel, S. R. Manmana, T. Pruschke, and A. Honecker. Matrix product state formulation of frequency-space dynamics at finite temperatures. Physical Review B 90, 060406(R) (2014). URL http://dx.doi.org/10.1103/PhysRevB.90.060406

$\left[\mathrm{THP}^{+} 16\right]$ A. C. Tiegel, A. Honecker, T. Pruschke, A. Ponomaryov, S. A. Zvyagin, R. Feyerherm, and S. R. Manmana. Dynamical properties of the sine-Gordon quantum spin magnet Cu-PM at zero and finite temperature. Physical Review B 93, 104411 (2016).

URL http://dx.doi.org/10.1103/PhysRevB.93.104411

$\left[\mathrm{KTF}^{+} 16\right] \quad$ E. S. Klyushina, A. C. Tiegel, B. Fauseweh, A. T. M. N. Islam, J. T. Park, B. Klemke, A. Honecker, G. S. Uhrig, S. R. Manmana, and B. Lake. Magnetic excitations in the $S=\frac{1}{2}$ antiferromagnetic-ferromagnetic chain compound $\mathrm{BaCu}_{2} \mathrm{~V}_{2} \mathrm{O}_{8}$ at zero and finite temperature. Physical Review B 93, 241109(R) (2016).

URL http://dx.doi.org/10.1103/PhysRevB.93.241109

Beyond this thesis, there also exist publications which are based on the author's bachelor's and master's theses. These have also been prepared during the period of $\mathrm{PhD}$ work and are listed as follows:

$\left[\mathrm{TDH}^{+} 13\right]$ A. C. Tiegel, P. E. Dargel, K. A. Hallberg, H. Frahm, and T. Pruschke. Spinspin correlations between two Kondo impurities coupled to an open Hubbard chain. Physical Review B 87, 075122 (2013).

URL http://dx.doi.org/10.1103/PhysRevB.87.075122

[TVD $\left.{ }^{+} 16\right]$ A. C. Tiegel, T. Veness, P. E. Dargel, A. Honecker, T. Pruschke, I. P. McCulloch, and F. H. L. Essler. Optical conductivity of the Hubbard chain away from half filling. Physical Review B 93, 125108 (2016).

URL http://dx.doi.org/10.1103/PhysRevB.93.125108 


\section{Table of contents}

Chapter 1 Introduction 1

1.1. Motivation and objectives ..................... 1

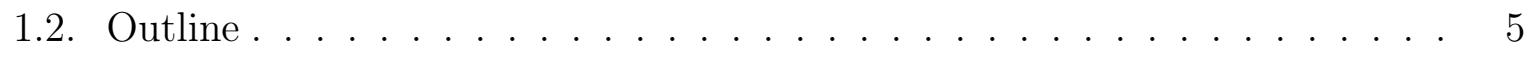

$\begin{array}{lll}\text { Chapter } 2 & \text { Spectral functions of many-body quantum systems } & 7\end{array}$

2.1. Many-body quantum states . . . . . . . . . . . . . . . . 7

2.2. Dynamical response functions . . . . . . . . . . . . . . . . . 9

2.2.1. Linear response theory . . . . . . . . . . . . . . . . . 9

2.2.2. Finite-temperature dynamical response . . . . . . . . . . . . . . . . 10

2.2.3. Zero-temperature dynamical response . . . . . . . . . . . . . . . . . 12

2.3. The density operator in Liouville space . . . . . . . . . . . . . . . . 13

2.4. Thermofield dynamics (TFD) . . . . . . . . . . . . . . . . . . 14

2.5. Frequency-space dynamics in Liouville space . . . . . . . . . . . . . 16

$\begin{array}{llr}\text { Chapter } 3 & \text { Computational methods } & 19\end{array}$

3.1. Introduction . . . . . . . . . . . . . . . . . . . . . . . 19

3.2. The Lanczos method for ground states . . . . . . . . . . . . . . . . 21

3.3. Matrix product states and the density-matrix renormalization group . . . . 22

3.3.1. Entanglement . . . . . . . . . . . . . . . . 22

3.3.2. Formalism of matrix product states (MPS) . . . . . . . . . . 25

3.3.3. Ground-state search . . . . . . . . . . . . . . . . . . 30

3.3.4. Variational compression of MPS . . . . . . . . . . . . . . 31

3.4. Time evolution . . . . . . . . . . . . . . . . . . 35

3.4.1. Spectral functions via real-time evolution . . . . . . . . . . . . 35

3.4.2. Time evolution via the time-dependent variational principle . . . . 36

3.4.3. Krylov-space time evolution . . . . . . . . . . . . . . . . . 38

3.4.4. Other time-evolution methods . . . . . . . . . . . . . . . 39

3.5. MPS frequency-domain methods at zero temperature . . . . . . . . . . 41

3.5.1. Introduction . . . . . . . . . . . . . . . . . . 41

3.5.2. Continued fraction expansion of spectral functions at $T=0$. . . 42

3.5.3. Expansions in Chebyshev polynomials . . . . . . . . . . . . . 42

3.5.4. Chebyshev expansion of spectral functions at $T=0 \ldots \ldots$. . . . 48

3.6. MPS frequency-domain methods at finite temperature . . . . . . . . . . 55

3.6.1. Introduction . . . . . . . . . . . . . . . . . . . . 55

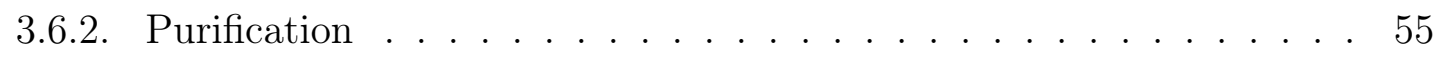

3.6.3. Dynamics of the purification . . . . . . . . . . . . 57

3.6.4. Chebyshev expansion of finite-temperature spectral functions . . . . 58

3.6.5. Continued fraction expansion at $T>0 \ldots \ldots$. . . . . . . 60 
$\begin{array}{lll}\text { Chapter } 4 \text { Models } & 61\end{array}$

4.1. Introduction . . . . . . . . . . . . . . . . . . . 61

4.2. The Heisenberg model . . . . . . . . . . . . . . . . . . . . . . . . . 62

4.2.1. The Heisenberg Hamiltonian . . . . . . . . . . . . . . . . . . 63

4.2.2. Excitations . . . . . . . . . . . . . . . . 64

4.2.3. Ferromagnetic Heisenberg model . . . . . . . . . . . . . . . 64

4.3. XX model . . . . . . . . . . . . . . . . . . . . 65

4.4. Superexchange interactions . . . . . . . . . . . . . . . 66

4.5. Dzyaloshinskii-Moriya interactions . . . . . . . . . . . . . . 67

4.5.1. Effective model . . . . . . . . . . . . . . . . . 68

4.5.2. Quantum sine-Gordon model . . . . . . . . . . . . . . 69

4.6. Alternating Heisenberg chain . . . . . . . . . . . . . . . . . . . 70

Chapter 5 Proof-of-principle MPS frequency-space calculations at $T>0 \quad 73$

5.1. Introduction . . . . . . . . . . . . . . . . . . . 73

5.2. Results for the $X X$ model . . . . . . . . . . . . . . . . . 74

5.2.1. Thermodynamics . . . . . . . . . . . . . . . 74

5.2.2. Reduced density-matrix spectra . . . . . . . . . . . . . . . 74

5.2.3. Dynamical spin structure factor . . . . . . . . . . . . . . 77

5.3. Results for the isotropic Heisenberg chain . . . . . . . . . . . . . . . 80

5.3.1. Thermodynamics in zero magnetic field . . . . . . . . . . . . . 80

5.3.2. Dynamical spin structure factor . . . . . . . . . . . . . . 82

5.4. Continued fraction expansion results . . . . . . . . . . . . . 85

5.5. Interim summary . . . . . . . . . . . . . . . . . . . 88

\section{Chapter 6 Dynamical properties of the sine-Gordon quantum spin} $\begin{array}{lr}\text { magnet } \mathrm{Cu}-\mathrm{PM} & 89\end{array}$

6.1. Introduction . . . . . . . . . . . . . . . . . . . . 89

6.2. The compound $\mathrm{Cu}-\mathrm{PM} \ldots \ldots \ldots \ldots 1$

6.2.1. Crystal structure and effective model . . . . . . . . . . . . . 91

6.2.2. Elementary excitations . . . . . . . . . . . . . . . . . . 92

6.3. ESR and mixing of components . . . . . . . . . . . . . . . . . . 92

6.4. Numerical details . . . . . . . . . . . . . . . . . . . . . . . . . . . . . . 93

6.4.1. DMRG calculations . . . . . . . . . . . . . . . 93

6.4.2. Exact diagonalization . . . . . . . . . . . . . . . . 94

6.5. Zero-temperature results . . . . . . . . . . . . . . . . . 94

6.5.1. BBS and breather excitations . . . . . . . . . . . 95

6.5.2. Single-soliton resonance . . . . . . . . . . . . . . . . . . 98

6.5.3. Comparison to the experiment . . . . . . . . . . . . . . . 100

6.5.4. Dynamical spin structure factor . . . . . . . . . . . . . . 101

6.6. Finite-temperature results . . . . . . . . . . . . . . . 103

6.6.1. Breather and interbreather excitations . . . . . . . . . . . 103

6.6.2. Soliton-breather transition . . . . . . . . . . . . . . . 107

6.6.3. Crossover: soliton to paramagnetic line . . . . . . . . . . . . 108

6.7. Conclusion . . . . . . . . . . . . . . . . . . . . 109 
Chapter 7 Magnetic excitations in the spin-1/2 dimerized-chain compound $\mathrm{BaCu}_{2} \mathrm{~V}_{2} \mathrm{O}_{8} \quad 111$

7.1. Introduction . . . . . . . . . . . . . . . . . . . 111

7.2. The compound $\mathrm{BaCu}_{2} \mathrm{~V}_{2} \mathrm{O}_{8} \ldots \ldots \ldots \ldots \ldots 114$

7.3. Identification of the Hamiltonian . . . . . . . . . . . . . 115

7.3.1. Static magnetic susceptibility . . . . . . . . . . . . . . . . 115

7.3.2. Magnetic excitation spectra at $T=0 \ldots \ldots$. . . . . . . 117

7.4. Finite-temperature results . . . . . . . . . . . . . 120

7.4.1. Correlation length . . . . . . . . . . . . . . . . 120

7.4.2. Temperature-induced asymmetric lineshape broadening . . . . . . . 121

7.4.3. Finite-temperature correlation functions . . . . . . . . . . . . . 128

7.4.4. Intraband excitations . . . . . . . . . . . . . . . . 130

7.5. Conclusion . . . . . . . . . . . . . . . . . . . . 132

$\begin{array}{lll}\text { Chapter } 8 & \text { Summary and conclusion } & 135\end{array}$

8.1. MPS formulation of frequency-space dynamics at finite temperature . . . . 135

8.2. Dynamical properties of the sine-Gordon quantum magnet $\mathrm{Cu}-\mathrm{PM}$. . . . . 136

8.3. Coherent quantum behavior at elevated temperatures . . . . . . . . . . . 137

8.4. Further perspectives . . . . . . . . . . . . . . . . . . 139

\section{Appendices}

Chapter A Further results for $\mathrm{Cu}-\mathrm{PM} \quad$ III

A.1. Frequency-field plots . . . . . . . . . . . . . . . III

A.2. Finite-size analysis of ED results . . . . . . . . . . . IV

Chapter B Further results for $\mathrm{BaCu}_{2} \mathrm{~V}_{2} \mathrm{O}_{8} \quad \mathrm{~V}$

B.1. Dimer susceptibility . . . . . . . . . . . . . . . . V

B.2. Intradimer correlation function . . . . . . . . . . . . . . VI

B.3. Temperature dependence of $J_{1}$ in $\mathrm{BaCu}_{2} \mathrm{~V}_{2} \mathrm{O}_{8} \ldots \ldots \ldots \ldots$. . . . . . . . . .

B.4. Linear prediction . . . . . . . . . . . . . . . . . . VIII

\section{List of references}





\section{\begin{tabular}{l|l} 
& \\
Introduction & 1
\end{tabular}}

Motivation and objectives $\mid 1.1$

Quasi-one-dimensional compounds of magnetic ions with low spin have received a lot of attention [MK04]. Such materials are crystals with highly anisotropic properties [LGG03]. This implies that below a certain threshold temperature the coupling of the magnetic spins in one spatial direction becomes dominant whereas the spin-spin interactions in the other two directions are negligible. In a first approximation, such systems can be effectively described by a single one-dimensional (1D) chain of coupled quantum spins localized at the ion positions. ${ }^{1}$ In this thesis, the corresponding magnetic moments are treated as Heisenberg spins [Hei28] for which the spin vector can point in any direction in threedimensional space. In the considered materials, the magnetic moments are realized as $\mathrm{Cu}^{2+}$ ions with a quantum mechanical spin of $S=1 / 2$. A prototypical compound well described by the Heisenberg model at low temperature is $\mathrm{KCuF}_{3}$ [TPCN93, TCNT95, LTFN05].

Such 1D quantum spin systems are particularly interesting for a multitude of reasons. At low temperatures, they exhibit enhanced quantum effects due to their reduced dimensionality [BD05]. In addition, quantum fluctuations are most pronounced for a small value of the spin. They vanish as a $1 / S$ effect in the classical limit $S \rightarrow \infty$. Furthermore, the spin-spin interactions lead to strong quantum correlations among them giving rise to, e.g., the suppression of long-range magnetic order and exotic ground-state excitations [Faz99, Blu01]. These phenomena are a manifestation of true many-body physics [FW71, Mah00] that cannot be captured by a theory treating the many degrees of freedom individually on the single-particle level.

Many-body problems are difficult to treat in the presence of strong correlations and still pose some of the hardest challenges in condensed matter physics. If one considers a chain of $L$ spin-1/2 degrees of freedom, each of them has a basis $\{|\uparrow\rangle,|\downarrow\rangle\}$. Therefore, the total dimension of the quantum mechanical state space is $2^{L}$. In order to describe macroscopic materials, one needs to consider the thermodynamic limit, i.e., $L \rightarrow \infty$, or at least finite systems that are sufficiently large. The latter route is pursued by the numerical calculations in this thesis. The treatment of such systems is difficult due the large dimensionality of the state space growing exponentially with system size. At absolute zero temperature $T=0$, there has been a lot of progress in the theoretical description of 1D quantum many body-systems. For instance, the exact analytical solution of the ground state of the 1D Heisenberg model is available via Bethe ansatz [Bet31, KMGT97, KHM98]. The structure of basic Bethe-ansatz eigenfunctions for finite systems is generally inconvenient for the calculation of dynamical quantities, which are mainly studied in this thesis. However, based on Bethe ansatz the exact calculation of the intensity of such quantities can be achieved for infinitely large systems and in special cases at $T=0$ [CH06]. Another

\footnotetext{
${ }^{1}$ Confer to Chapter 4 for a more detailed discussion of the considerations leading to such a model.
} 
class of analytical approaches based on field theory makes approximations [Tsv07, Fra13]. Their quality is usually hard to ascertain. Therefore, numerical studies as presented in this thesis are very useful both as a means of verification of analytical results and to gain new insights in cases where an analytical treatment is not available.

Studying dynamical observables of strongly correlated quantum spin systems is of great interest as they provide important insights into the governing many-body physics [FW71, Mah00]. In contrast to static properties, dynamical response functions contain more information about the system and its excitations.

The main focus of this thesis is the numerical calculation of dynamical quantities of 1D spin chains at finite temperatures $T>0$. This is largely motivated by recent experimental advances in neutron scattering [ZL05, ZT15] and electron spin resonance [Zvy12] allowing for very precise high-resolution measurements of dynamical quantities in quasi-1D quantum magnets. Importantly, thermal fluctuations may lead to new phenomena occurring upon tuning the temperature. Examples are temperature-induced transitions between ground-state excitations [ $\left.\mathrm{UTO}^{+} 09\right]$, or the quantum critical regime in some strongly correlated materials [Sac01].

This implies that the availability of efficient and accurate numerical tools for making theoretical predictions of dynamical observables at finite temperature is highly desirable. One main part of this thesis is concerned with the development of such a numerical method.

The numerical techniques used in this thesis are based on the density-matrix renormalization group (DMRG) [Sch05a] in which the quantum mechanical wave function assumes the form of a matrix product state (MPS) [Sch11]. The methods employed are formulated and implemented in the framework of MPS in this thesis. Concerning the terminology, in the following they are referred to as MPS approaches or - in the broadest sense - simply as DMRG. These methods have arguably been the most powerful numerical techniques in the study of quantum chains. Initially, the original DMRG algorithm aimed at the calculation of ground-state properties [Whi92, Whi93]. The idea behind the algorithm is to truncate the many-body basis of the Hilbert space by a variational principle. It has been extended to the accurate calculation of both frequency- and momentum-resolved spectral functions of $1 \mathrm{D}$ systems at zero-temperature. These methods can be categorized according to the route they pursue for such calculations. On one hand, spectral functions may be computed directly in the frequency domain, e.g., by moment expansions [Hal95, $\left.\mathrm{DWH}^{+} 12, \mathrm{HWM}^{+} 11\right]$. On the other hand, they can also be obtained via the calculation of time-domain correlation functions by DMRG-based real-time evolution [Vid04, WF04, DKSV04] followed by a Fourier transform to frequency space. These zerotemperature techniques are well established and the time-domain approaches have already been extended to $T>0$ [BSW09, KBM12]. The challenge is that finite-temperature states generally need to be described by mixed-state density operators. However, MPS can in principle only represent pure-state wave functions. This problem can be solved via a purification of the thermal density operator obtained by doubling the degrees of freedom leading to an enlarged system of twice the size of the physical system. The advantage is that the finite-temperature state can be represented by a wave function and thus by an MPS again [Sch11]. Then the finite-temperature dynamics is formulated in Liouville space [Fan57, BD87]. The first goal of this thesis is to use the existing formulation in order to devise an MPS-based frequency-domain method for the calculation of finite-temperature spectral functions. In this way, state-of-the-art zero-temperature MPS methods working in the frequency domain will be extended to finite temperatures in the present thesis. 
It may also be mentioned that apart from the DMRG methods used in this thesis there are other established numerical methods for the calculation of dynamical properties in 1D quantum systems at $T>0$ such as, e.g., full diagonalization [NM05] or quantum Monte Carlo (QMC) methods [San10]. In the first approach, the Hamilton operator is represented as a matrix which is diagonalized in order to gain access to the full spectrum of its eigenenergies and the corresponding eigenstates. From this information dynamical observables can be calculated. However, the method is limited to rather small systems only. QMC methods rely on stochastic sampling and are thus also applicable to larger systems, but the technique is restricted by a challenging analytical continuation procedure to calculate spectral functions. Depending on the system, the negative sign problem can also pose a problem $\left[\mathrm{GML}^{+} 11\right]$.

Having pointed out the general interest in $1 \mathrm{D}$ systems and the numerical methods that will be used, the following remarks are intended to give a brief introduction to the two systems mainly studied in this work. The newly developed MPS method will be applied to the calculation of their dynamical properties at finite temperature. The calculation of zero-temperature spectral functions with existing MPS approaches is another essential part of this thesis.

For the prototypical compound $\mathrm{KCuF}_{3}$ which is well described by the Heisenberg model, experimental results obtained by inelastic neutron scattering (INS) at low temperature are in good agreement [LTFN05] with the model which has a gapless continuum of spin excitations at $T=0$ [FT81, MTBB81]. However, in many 1D quantum magnets the antiferromagnetic Heisenberg Hamiltonian is not a good approximation because of further or different exchange interactions between the magnetic ions. Two quasi-1D materials of this type are mainly studied in this thesis.

The first system includes additional but weak Dzyaloshinskii-Moriya (DM) interactions [Dzy58] presenting a perturbation to the Heisenberg Hamiltonian. These antisymmetric interactions originate from the spin-orbit coupling [Mor60a] in crystals with alternating crystal axes. The staggered DM interaction is present in some anisotropic $\mathrm{Cu}^{2+}$ based compounds, e.g., in copper benzoate [DYMT70] and copper pyrimidine dinitrate $\left(\left[\mathrm{PM} \cdot \mathrm{Cu}\left(\mathrm{NO}_{3}\right)_{2} \cdot\left(\mathrm{H}_{2} \mathrm{O}\right)_{2}\right]_{n}, \mathrm{PM}=\right.$ pyrimidine; or shortly $\left.\mathrm{Cu}-\mathrm{PM}\right)\left[\mathrm{INN}^{+} 97, \mathrm{FAG}^{+} 00\right]$. The latter material is studied in this thesis. In a uniform applied magnetic field, $\mathrm{Cu}-\mathrm{PM}$ displays a field-induced gap in the magnetic excitation spectra probed by INS [DHR $\left.{ }^{+} 97\right]$. The gap can be attributed to an effective transverse staggered field induced by both the DM interactions [OA97] and a staggered $g$ tensor describing the anisotropic coupling between the applied magnetic field and the spins. Both effects occur as a consequence of the alternating arrangement of the copper ions [DHR ${ }^{+97]}$. Although the magnitude of these two effects is only a few percent compared to the Heisenberg exchange coupling, the field-induced gap represents a striking departure from the gapless spin excitations of the Heisenberg model. The effective Hamiltonian consisting of the Heisenberg terms and the additive contributions including the uniform and staggered magnetic fields can be mapped to a quantum sine-Gordon field theory [OA97, ET98, AO99] which is valid at zero temperature and low energy. Materials such as $\mathrm{Cu}-\mathrm{PM}$ are thus referred to as sine-Gordon magnets. Their low-energy elementary excitations at zero temperature can be effectively described by this field theory. The main elementary excitations are solitons and antisolitons as well as their bound states. This was confirmed in electron spin resonance measurements (ESR) at low $T$ for the compound $\mathrm{Cu}-\mathrm{PM}$ [ZKKF04, ZCO $\left.{ }^{+} 11\right]$. Despite the good agreement, there remain open questions at $T=0$. These are mainly due to deviations from the field theory and recently reported boundary states [FO12]. Zero- 
temperature calculations of dynamical properties with state-of-the-art MPS methods are therefore one goal of this thesis. They will offer new insights into these issues.

Moreover, the study of temperature effects in sine-Gordon quantum magnets is a focal point of interest and a main objective of this thesis. This is motivated by hints of strong temperature dependencies in ESR experiments on Cu-PM [Zvy12]. Moreover, there are still a few experimentally unobserved field-theoretical predictions for thermally-induced transitions between elementary excitations [FO12]. These are specific examples demonstrating the need for accurate and reliable numerical calculations of finite-temperature spectral functions.

Furthermore, another main question of this thesis is concerned with the persistence of the strongly correlated quantum behavior observed at low $T$ with increasing temperature. Upon raising the temperature, there develops an interesting interplay of quantum and thermal fluctuations. The latter will lead to a crossover to the classical high-temperature regime in which the quantum mechanical coherence is lost. The persistence of quantum behavior at higher temperatures is of practical importance for potential applications in quantum devices. Also in the fundamental study of quantum coherence, highly anisotropic $\mathrm{Cu}^{2+}$-based compounds have offered interesting insights. In particular, quasi-1D crystals with two inequivalent exchange paths are considered in this context.

A paradigmatic example is the material copper nitrate $\left[\mathrm{TLJ}^{+} 12\right]$. Another realization with unexplored dynamical properties is the material $\mathrm{BaCu}_{2} \mathrm{~V}_{2} \mathrm{O}_{8}$ which represents the second system studied in this thesis. It can be modeled by an alternating spin- $1 / 2$ Heisenberg chain in which the spins are coupled by two different exchange couplings $J$ and $J^{\prime}$ in an alternating fashion. For $\mathrm{BaCu}_{2} \mathrm{~V}_{2} \mathrm{O}_{8}$, one has $J \gg J^{\prime}$ which leads to a strong dimerization of the system. Then two neighboring spins coupled by the antiferromagnetic $J$ form dimers which are only weakly coupled by $J^{\prime}$. The model has a spin-singlet ground state and gapped triplet excitations which are triplons. There are strong correlations between these excitations.

In copper nitrate, the single-triplon mode is subject to asymmetric lineshape broadening with increasing temperature as observed in inelastic neutron scattering (INS) experiments $\left[\mathrm{TLJ}^{+} 12\right]$. This is in contradiction to the conventional description of temperature effects in quantum magnets [ML71] which predicts symmetric lineshape broadening. However, this theory is based on weak interactions among the excitations. Asymmetric lineshape broadening has also been predicted by integrable quantum field theories [EK08, EK09] and full diagonalization for small dimerized systems [ML06, TLJ $\left.{ }^{+} 12\right]$.

Concerning the study of $\mathrm{BaCu}_{2} \mathrm{~V}_{2} \mathrm{O}_{8}$, the Hamiltonian of this compound is identified by a combination of INS experiments and MPS calculations. Based on this model, the asymmetric lineshape broadening in $\mathrm{BaCu}_{2} \mathrm{~V}_{2} \mathrm{O}_{8}$ is investigated by means of the finite-temperature MPS method developed in the present work. The results obtained in this thesis are compared to further experimental INS data and a diagrammatic approach [FSU14, FU15]. 


\section{\begin{tabular}{l|l} 
Outline & 1.2
\end{tabular}}

After motivating the objectives of this thesis, its outline is given below:

Chapter 2 starts by presenting some basic aspects of many-body quantum physics which are relevant for the following chapters. Then dynamical response functions, which will be mainly calculated, are briefly reviewed and formulated in Liouville space which is conceptually important for the finite-temperature method developed in this thesis.

Chapter 3 provides information on the computational methods employed in the present work. After introducing matrix product states (MPS), the density-matrix renormalization group (DMRG) and some of its extensions for the computation of dynamical properties are reviewed in the framework of MPS. Special emphasis is put on existing frequency-domain methods for the calculation of spectral functions at $T=0$, see Sec. 3.5. In Sec. 3.6, the MPS method developed in this thesis is presented by formulating these frequency-domain methods at finite temperature by exploiting a Liouville-space formulation.

In Chapter 4, the models studied in this thesis are motivated and reviewed.

First proof-of-principle MPS frequency-domain computations of finite-temperature spectral functions are shown in Chapter 5. The newly developed method is characterized and its potential is assessed - both by a comparison to exact results for the $1 \mathrm{D} X X$ model and a treatment of spin-1/2 Heisenberg chains with Dzyaloshinskii-Moriya interactions mimicked by a transverse staggered field. Moreover, static thermodynamic observables are computed by MPS imaginary-time evolution.

In Chapter 6, both the frequency-field dependence and the intensity of the elementary excitations in the sine-Gordon magnet copper pyrimidine dinitrate $(\mathrm{Cu}-\mathrm{PM})$ is investigated at zero and finite temperature. The MPS results obtained in this thesis are compared to exact diagonalization, predictions by sine-Gordon field theories, and electron spin resonance (ESR) experiments.

In Chapter 7, the persistence of strongly correlated quantum behavior at elevated temperatures is studied in the dimer-chain compound $\mathrm{BaCu}_{2} \mathrm{~V}_{2} \mathrm{O}_{8}$. Here the main focus is on the quantitative prediction of the asymmetric lineshape broadening occurring due to these strong correlations. To this end, the finite-temperature spectral functions calculated within this thesis are compared to different results obtained by inelastic neutron scattering (INS) and a diagrammatic approach.

Finally, Chapter 8 contains a concluding summary of the main results of this thesis and opens perspectives for future research. 



\section{Spectral functions of many-body quantum systems}

Instead of providing a full and detailed review of many-body quantum physics [Mah00, Pru14], the goal of this chapter is to briefly introduce the main concepts that are relevant for the further reading of this thesis. A particular focus is the definition of spectral functions both at zero and finite temperature. Then a Liouville-space formulation and thermofield dynamics are reviewed in order to express finite-temperature spectral functions in a form amenable to the numerical approach developed in this thesis.

\section{\begin{tabular}{l|l} 
Many-body quantum states & 2.1
\end{tabular}}

The systems studied in this work are 1D spin-1/2 models defined on lattices consisting of a finite number $L$ of sites. ${ }^{1}$ Therefore, the Hilbert space dimension is assumed to be finite in the following. For spin- $1 / 2$ degrees of freedom, the local Hilbert space $\mathcal{H}_{l}^{\text {loc }}$ at each site $l$ is spanned by the basis set $\{|\downarrow\rangle,|\uparrow\rangle\}$ and is thus of dimension $d=2$. The total Hilbert space is obtained as the tensor product space

$$
\mathcal{H}^{\mathrm{tot}}=\bigotimes_{l=1}^{L} \mathcal{H}_{l}^{\mathrm{loc}}
$$

yielding a dimension of

$$
\operatorname{dim}\left(\mathcal{H}^{\text {tot }}\right)=d^{L}
$$

The fact that this dimension grows exponentially with system size $L$ poses a severe challenge for computational methods implemented on binary computers, as used in this thesis.

Any pure quantum mechanical state can be described by a vector $|\Psi\rangle$ which is an element of the Hilbert space spanned by an arbitrary orthonormal basis set $\left\{\left|u_{n}\right\rangle\right\}$ :

$$
|\Psi\rangle=\sum_{n}\left\langle u_{n} \mid \Psi\right\rangle\left|u_{n}\right\rangle=\sum_{n} c_{n}\left|u_{n}\right\rangle
$$

The coefficients $c_{n}$ of this state vector are generally complex numbers and a pure state is referred to as a wave function.

In this thesis, the Hamiltonian $H$ describing the physical system is always assumed to be time-independent. The wave functions of stationary states are the solutions of the time-independent Schrödinger equation

$$
H|\Psi\rangle=E|\Psi\rangle
$$

\footnotetext{
${ }^{1}$ Confer to Chapter 4 for a discussion of the considerations leading to models of this type.
} 
This equation represents an eigenvalue problem. Therefore, the stationary states are also called energy eigenstates $\left|E_{n}\right\rangle$ with energies $E_{n}=\left\langle E_{n}|H| E_{n}\right\rangle /\left\langle E_{n} \mid E_{n}\right\rangle$. The eigenstates form an orthonormal basis set $\left\{\left|E_{n}\right\rangle\right\}$ in Hilbert space. ${ }^{2}$ Represented with respect to this basis, the Hamilton operator becomes diagonal. At $T=0$, a system in equilibrium is in its ground state $\left|E_{0}\right\rangle$ with minimal energy $E_{0}$. As we will see in Sec. 2.2.2 below, in the definition of spectral functions the eigenstates and the discrete eigenspectrum of the Hamiltonian play an important role.

The dynamics of a quantum mechanical system is governed by the time-dependent Schrödinger equation. For a pure quantum state it reads

$$
i \hbar \frac{d}{d t}|\Psi(t)\rangle=H|\Psi(t)\rangle
$$

and has the formal solution

$$
|\Psi(t)\rangle=U\left(t-t_{0}\right)\left|\Psi\left(t_{0}\right)\right\rangle, \quad U\left(t-t_{0}\right)=e^{-i H\left(t-t_{0}\right) / \hbar},
$$

where $U\left(t-t_{0}\right)$ is the unitary time-evolution operator and $t_{0}$ the initial time.

At finite temperature, the description of quantum states which are represented by state vectors is not sufficient since the system is generally in a mixed state, i.e., in a statistical mixture of pure states $\left|\Psi_{i}\right\rangle$ each occurring with a probability $p_{i}>0$. In order to deal with this probabilistic ensemble, the density matrix describing a mixed state is defined as the linear operator

$$
\rho=\sum_{i} p_{i}\left|\Psi_{i}\right\rangle\left\langle\Psi_{i}\right|
$$

The density operator $\rho$ of a mixed state is a superposition of pure-state density operators $\left|\Psi_{i}\right\rangle\left\langle\Psi_{i}\right|$. The condition $\sum_{i} p_{i}=1$ grants the normalization of $\rho$, i.e., $\operatorname{Tr} \rho=1$. The equilibrium expectation value of an operator $A$ can thus be stated as

$$
\langle A\rangle=\operatorname{Tr}(\rho A) .
$$

At finite temperature $T>0$, the canonical density operator reads

$$
\rho=\frac{e^{-\beta H}}{\operatorname{Tr} e^{-\beta H}} .
$$

The partition function is defined as $Z=\operatorname{Tr} e^{-\beta H}=\sum_{n} \mathrm{e}^{-\beta E_{n}}$ and $\beta=\left(k_{\mathrm{B}} T\right)^{-1}$ denotes the inverse temperate, where $k_{\mathrm{B}}$ is the Boltzmann constant. Thus, the expectation value in the canonical ensemble is given by

$$
\langle A\rangle=\frac{1}{Z} \operatorname{Tr}\left(e^{-\beta H} A\right)=\frac{1}{Z} \sum_{n} \mathrm{e}^{-\beta E_{n}}\left\langle E_{n}|A| E_{n}\right\rangle .
$$

Moreover, the time-dependence of arbitrary mixed quantum states as introduced in Eq. (2.7) is described by the equation (see, e.g., Ref. [Muk95])

$$
i \hbar \frac{d}{d t} \rho(t)=[H, \rho(t)]
$$

It is known as the quantum Liouville equation or alternatively Liouville-von Neumann equation and represents an extension of Eq. (2.5) to arbitrary quantum states.

\footnotetext{
${ }^{2}$ Due to the finite dimension of the Hilbert space assumed earlier, the basis set also remains finite.
} 


\section{\begin{tabular}{l|l} 
Dynamical response functions & 2.2
\end{tabular}}

In the following, a simplified situation motivated by experiments is used as a starting point for the definition of dynamical response functions. Then the frequency-domain representation of such quantities is derived following Refs. [Mah00, Pru14] in Sec. 2.2.2. The representation is used to define spectral functions which play a central role in this thesis. The presentation below is also based on Ref. [CL00]. ${ }^{3}$

\section{\begin{tabular}{l|l} 
Linear response theory & 2.2 .1
\end{tabular}}

In order to investigate a solid in experiments, one frequently applied procedure is to introduce a weak time-dependent external perturbation $V(t)$. Then the total Hamiltonian is of the form

$$
H_{\text {tot }}=H+V(t) .
$$

Here $H$ denotes the time-independent Hamiltonian of the unperturbed system. The perturbation is switched on at a time $t_{0}$. For $t<t_{0}$, the system is assumed to be in thermal equilibrium. An exemplary perturbation is given by

$$
V(t)=-B f(t),
$$

where $B$ is an operator representing an observable of the system (i.e. $B=B^{\dagger}$ ) and $f(t)$ denotes a real-valued applied field which has no spatial dependence for simplicity.

A common way to treat the dynamical response of the system to a small external perturbation, is linear response theory [Pru14]. In this theory, the thermal expectation value $\langle A\rangle(t)$ of a further operator $A$ with respect to $H_{\text {tot }}$ is calculated up to linear order in the field $f(t)$ in time-dependent perturbation theory [SN11]. This gives a so-called Kubo formula [Kub57]

$$
\langle A\rangle(t)=\langle A\rangle+\int_{-\infty}^{\infty} \mathrm{d} t^{\prime} \chi_{A B}\left(t-t^{\prime}\right) \cdot f\left(t^{\prime}\right),
$$

in which the dynamical response function $\chi_{A B}\left(t-t^{\prime}\right)$ is defined as

$$
\chi_{A B}\left(t-t^{\prime}\right):=\frac{i}{\hbar} \Theta\left(t-t^{\prime}\right)\left\langle\left[A(t), B\left(t^{\prime}\right)\right]\right\rangle .
$$

Note that the average in Eq. (2.15) is taken only with respect to the unperturbed Hamiltonian $H$. Moreover, $\Theta\left(t-t^{\prime}\right)$ represents the unit step function. The importance of Eq. (2.14) is that it relates the changes $\delta(\langle A\rangle(t))=\langle A\rangle(t)-\langle A\rangle$ in the expectation values of the observable $A$ to the weak time-dependent external perturbation, where $\langle A\rangle$ denotes the equilibrium expectation value at $t<t_{0}$, see Eq. (2.8). Thus, the quantity $\chi_{A B}\left(t-t^{\prime}\right)$ is important for the description of experiments and is also referred to as dynamical susceptibility. Causality is ensured by the condition $t>t^{\prime}$. In the following $\hbar=1$ is adopted.

\footnotetext{
${ }^{3}$ For further reading also confer to Refs. [BF04, Eco06].
} 


\section{Finite-temperature dynamical response}

Here finite-temperature spectral functions are formulated in terms of the previously defined dynamical response functions. From a mathematical point of view, it will turn out that there is a very close relation between a spectral function for a given observable and the Fourier transform of the corresponding dynamical response function in Eq. (2.15). Since finite temperatures are considered here, the average in Eq. (2.15) denotes the thermodynamic average. In order to obtain a particular representation for the response function, the canonical ensemble is used in the following. Moreover, it is justified to set $t^{\prime}=0$ as $\chi_{A B}$ only depends on the difference for a time-independent Hamiltonian $H$ with eigenstates $\left|E_{n}\right\rangle$. Then a dynamical response function is cast in the following form:

$$
\begin{aligned}
\chi_{A B}(t) & =i \Theta(t) \frac{1}{Z} \sum_{n}\left\langle E_{n}\left|\mathrm{e}^{-\beta H}(A(t) B-B A(t))\right| E_{n}\right\rangle \\
& =i \Theta(t) \frac{1}{Z} \sum_{n} \mathrm{e}^{-\beta E_{n}}\left\langle E_{n}|(A(t) B-B A(t))| E_{n}\right\rangle .
\end{aligned}
$$

Inserting two identities $I=\sum_{m}\left|E_{m}\right\rangle\left\langle E_{m}\right|$ and treating the time dependence of an operator $A$ in the Heisenberg picture via

$$
\left\langle E_{n}|A(t)| E_{m}\right\rangle=\left\langle E_{n}\left|\mathrm{e}^{i H t} A \mathrm{e}^{-i H t}\right| E_{m}\right\rangle=\mathrm{e}^{i\left(E_{n}-E_{m}\right) t}\left\langle E_{n}|A| E_{m}\right\rangle
$$

yields

$$
\begin{aligned}
\chi_{A B}(t)= & \frac{i \Theta(t)}{Z} \sum_{m, n} \mathrm{e}^{-\beta E_{n}}\left[\left\langle E_{n}|A(t)| E_{m}\right\rangle\left\langle E_{m}|B| E_{n}\right\rangle-\left\langle E_{n}|B| E_{m}\right\rangle\left\langle E_{m}|A(t)| E_{n}\right\rangle\right] \\
= & \frac{i \Theta(t)}{Z} \sum_{m, n} \mathrm{e}^{-\beta E_{n}}\left[\mathrm{e}^{i t\left(E_{n}-E_{m}\right)}\left\langle E_{n}|A| E_{m}\right\rangle\left\langle E_{m}|B| E_{n}\right\rangle\right. \\
& \left.-\mathrm{e}^{-i t\left(E_{n}-E_{m}\right)}\left\langle E_{n}|B| E_{m}\right\rangle\left\langle E_{m}|A| E_{n}\right\rangle\right]
\end{aligned}
$$

In the second term, the indices $m$ and $n$ can be exchanged which leads to the following representation of the dynamical response function:

$$
\chi_{A B}(t)=i \Theta(t) \frac{1}{Z} \sum_{m, n}\left\langle E_{n}|A| E_{m}\right\rangle\left\langle E_{m}|B| E_{n}\right\rangle \mathrm{e}^{i t\left(E_{n}-E_{m}\right)}\left[\mathrm{e}^{-\beta E_{n}}-\mathrm{e}^{-\beta E_{m}}\right] .
$$

Now it is desirable to obtain a frequency-domain representation of this expression as well since many solid-state experiments give frequency-resolved results. The retarded dynamical susceptibility is obtained by the Fourier transform

$$
\begin{aligned}
\chi_{A B}^{\mathrm{ret}}(z=\omega+i \eta) & =\int_{-\infty}^{\infty} \mathrm{d} t \mathrm{e}^{i z t} \chi_{A B}(t) \\
& =-\frac{1}{Z} \sum_{m, n}\left\langle E_{n}|A| E_{m}\right\rangle\left\langle E_{m}|B| E_{n}\right\rangle \frac{\mathrm{e}^{-\beta E_{n}}-\mathrm{e}^{-\beta E_{m}}}{\omega+i \eta+E_{n}-E_{m}} .
\end{aligned}
$$

Here a small real number $\eta>0$ is introduced to shift the poles of the integrand from the real axis into the upper half of the complex plane in order to ensure the convergence of the 
integral. Using the frequency-domain expression for the response function in Eq. (2.23), a spectral function can be defined as

$$
R(\omega)=\lim _{\eta \rightarrow 0} \frac{1}{\pi} \operatorname{Im} \chi_{A B}^{\mathrm{ret}}(\omega+i \eta) .
$$

The identity

$$
\frac{1}{x \pm i \eta} \rightarrow \mathcal{P} \frac{1}{x} \mp i \pi \delta(x) \quad \text { for } \quad \eta \rightarrow 0
$$

is useful for identifying the imaginary part of $\chi_{A B}^{\mathrm{ret}}(\omega+i \eta)$, which is also written as $\chi_{A B}^{\mathrm{ret}}$ " . Here $\mathcal{P}$ denotes the Cauchy principal value. The result

$$
\begin{aligned}
R_{A B}(\omega) & =\frac{1}{Z} \sum_{m, n}\left(\mathrm{e}^{-\beta E_{n}}-\mathrm{e}^{-\beta E_{m}}\right)\left\langle E_{n}|A| E_{m}\right\rangle\left\langle E_{m}|B| E_{n}\right\rangle \delta\left(\omega-\left(E_{m}-E_{n}\right)\right) \\
& =\frac{1}{Z}\left(1-\mathrm{e}^{-\beta \omega}\right) \sum_{m, n} \mathrm{e}^{-\beta E_{n}}\left\langle E_{n}|A| E_{m}\right\rangle\left\langle E_{m}|B| E_{n}\right\rangle \delta\left(\omega-\left(E_{m}-E_{n}\right)\right)
\end{aligned}
$$

is known as the Lehmann representation of the spectral function. ${ }^{4}$ This representation is very instructive as it reveals the excitation energies $\omega_{m, n}=\left(E_{m}-E_{n}\right)$ of the system since they appear in the arguments of the Dirac delta functions. Moreover, their spectral weight is expressed by the corresponding matrix elements. In order to determine the spectral function via Eq. (2.27), a huge number of general matrix elements would have to be calculated. Therefore, its term-by-term calculation via the Lehmann representation is generally rather challenging for interacting systems and will not be used for the densitymatrix renormalization group (DMRG) calculations in the present work.

Furthermore, the finite systems studied in this thesis (see Sec. 2.1) always possess a discrete and finite set of excitation energies. In order to conceal the resulting finite-size effects, the value of $\eta$ should be chosen large enough in practical DMRG calculations [Jec02, BS14]. A finite $\eta>0$ smears out the delta functions by introducing a Lorentzian broadening.

The numerical calculation of the dynamical spin structure factor is of central interest in this thesis as it can be probed in inelastic neutron scattering experiments. The dynamical spin structure factor is a both momentum- and frequency-resolved quantity which can be defined as

$$
\begin{aligned}
S_{\alpha \gamma}(\omega) & :=\frac{1}{\pi} \frac{\chi_{\alpha \gamma}^{\mathrm{ret}^{\prime \prime}}(\omega)}{1-\mathrm{e}^{-\beta \omega}} \\
& =\frac{1}{Z} \sum_{m, n} \mathrm{e}^{-\beta E_{n}}\left\langle E_{n}\left|S_{q}^{\alpha}\right| E_{m}\right\rangle\left\langle E_{m}\left|S_{q}^{\gamma}\right| E_{n}\right\rangle \delta\left(\omega-\left(E_{m}-E_{n}\right)\right) .
\end{aligned}
$$

Here $S_{q}^{\alpha}$ and $S_{q}^{\gamma}$ denote the Fourier transform of local spin operators $S_{i}^{\alpha}$ or $S_{i}^{\gamma}$ defined in real space. Their site indices are denoted by $i$ respectively $j$, whereas $q$ is the momentum. For spin-1/2 systems, they will be introduced in Sec. 4.2.1. Whenever the investigated system has symmetries, different components of the dynamical structure factor can be related. It is hence not necessary to study all nine components.

Furthermore, the imaginary part $\chi_{\alpha \gamma}^{\text {ret" }}(\omega)$ in the long-wavelength limit $q \rightarrow 0$ is proportional to the absorption intensity measured in electron spin resonance experiments which will be further discussed in Sec. 6.3.

\footnotetext{
${ }^{4}$ In the last step, the temperature factors are rearranged, using $\mathrm{e}^{-\beta E_{n}}\left(1-\mathrm{e}^{-\beta\left(E_{m}-E_{n}\right)}\right)$.
} 


\section{Zero-temperature dynamical response $\mid 2.2 .3$}

A zero-temperature dynamical response function can be derived if the canonical average in Eq. (2.15) is replaced by a simple ground-state average. A subsequent Fourier transform via Eq. (2.22) to frequency space yields

$$
\begin{aligned}
\chi_{A B}^{\mathrm{ret}, T=0}(z) & =-\sum_{n}\left[\frac{\left\langle E_{0}|A| E_{n}\right\rangle\left\langle E_{n}|B| E_{0}\right\rangle}{\omega+i \eta-\left(E_{0}-E_{n}\right)}-\frac{\left\langle E_{0}|B| E_{n}\right\rangle\left\langle E_{n}|A| E_{0}\right\rangle}{\omega+i \eta+E_{0}-E_{n}}\right] \\
& =-\left\langle E_{0}\left|A \frac{1}{\omega+i \eta-\left(H-E_{0}\right)} B\right| E_{0}\right\rangle+\left\langle E_{0}\left|B \frac{1}{\omega+i \eta+\left(H-E_{0}\right)} A\right| E_{0}\right\rangle,
\end{aligned}
$$

where again $z=\omega+i \eta$. There are two expressions, one for positive and the other one for negative frequencies. In this thesis, they are also referred to as dynamical correlation functions. The Lehmann representation of the associated zero-temperature spectral function $^{5}$ is then given by

$$
R_{A B}^{T=0}(\omega)=\left\{\begin{array}{rr}
-\sum_{n}\left\langle E_{0}|B| E_{n}\right\rangle\left\langle E_{n}|A| E_{0}\right\rangle \delta\left(\omega+\left(E_{n}-E_{0}\right)\right), \quad \omega<0 \\
\sum_{n}\left\langle E_{0}|A| E_{n}\right\rangle\left\langle E_{n}|B| E_{0}\right\rangle \delta\left(\omega-\left(E_{n}-E_{0}\right)\right), \quad \omega>0
\end{array}\right.
$$

While focusing on, e.g., positive frequencies, one also obtains the following representation by inserting an identity: ${ }^{6}$

$$
\begin{aligned}
R_{A B}^{T=0}(\omega>0) & =\sum_{n, m}\left\langle E_{0}|A| E_{m}\right\rangle \underbrace{\left\langle E_{m} \mid E_{n}\right\rangle}_{\delta_{m n}}\left\langle E_{n}|B| E_{0}\right\rangle \delta\left(\omega-\left(E_{n}-E_{0}\right)\right) \\
& =\sum_{m, n}\left\langle E_{0}|A| E_{m}\right\rangle\left\langle E_{m}\left|\delta\left(\omega-\left(H-E_{0}\right)\right)\right| E_{n}\right\rangle\left\langle E_{n}|B| E_{0}\right\rangle \\
& =\left\langle E_{0}\left|A \delta\left(\omega-\left(H-E_{0}\right)\right) B\right| E_{0}\right\rangle .
\end{aligned}
$$

In order to obtain the expression in Eq. (2.34), one uses that

$$
\left\langle E_{m}\left|\delta\left(\omega-\left(H-E_{0}\right)\right)\right| E_{n}\right\rangle=\delta\left(\omega-\left(E_{n}-E_{0}\right)\right) \delta_{m n}
$$

holds for the matrix elements. This is justified as the Hamiltonian is diagonal in its discrete eigenbasis. Apart from the discreteness, also the finite length of the basis is important and follows from the finite dimension of the Hilbert space for the systems studied here.

The numerical calculation of zero-temperature spectral functions in this thesis proceeds by considering the expression for $\omega>0$ or $\omega<0$ separately. For instance, the unbroadened spectral function in Eq. (2.35) is the starting point for an approximation via MPS-based Chebyshev expansions using the kernel polynomial method (KPM), for which a finite broadening is introduced subsequently (see Sec. 3.5.4). Other zero-temperature DMRG approaches such as a related variant of Chebyshev expansions [BS14] or a continued fraction expansion (see Sec. 3.5.2) evaluate the broadened resolvent expression for

\footnotetext{
${ }^{5}$ The spectral function at $T=0$ is also obtained via the definition in Eq. (2.24).

${ }^{6}$ The result in Eq. (2.35) will be reused in Sec. 2.5.
} 
either positive or negative frequencies as occurring in Eq. (2.31).

Furthermore, the dynamical spin structure factor at $T=0$ is given by

$$
S_{\alpha \gamma}^{T=0}(\omega)=R_{\alpha \gamma}^{T=0}(\omega)=\sum_{n}\left\langle E_{0}\left|S_{q}^{\alpha}\right| E_{n}\right\rangle\left\langle E_{n}\left|S_{q}^{\gamma}\right| E_{0}\right\rangle \delta\left(\omega-\left(E_{n}-E_{0}\right)\right) .
$$

Comparing the zero- and finite-temperature dynamical structure factor in Eqs. (2.29) and (2.37), one realizes that the calculation at $T=0$ is simplified as there is only a single sum. Moreover, the matrix elements are less general as each of them involves the ground state. The comparison illustrates the challenges encountered particularly at $T>0$.

\section{The density operator in Liouville space $\mid 2.3$}

So far, the density operator and the governing equation of its dynamics (see Eq. (2.11)) have been briefly introduced. At this point, it is useful to make the preliminary comment that the density-matrix renormalization group (DMRG) techniques used in this thesis inherently only allow for the treatment of pure states since their underlying variational ansatz class are matrix product states (MPS). However, in finite-temperate calculations the representation of mixed quantum states cannot be avoided. Now an alternative formulation of how to consider the density operator representing such states is introduced. Fortunately, this formulation in Liouville space will render practical calculations involving a density operator formally identical to calculations for a wave function. This is one established procedure granting the applicability of DMRG methods at finite temperatures [Sch11]. In the following, the formulation of dynamics in Liouville space is introduced largely following Ref. [Muk95]. The Liouville space was introduced by Fano [Fan57].

First, a very brief introduction to the states and linear operators in Liouville space is given. This is instructive to develop a rough idea of the underlying mathematical structures. However, these will not be used for actual calculations in this thesis. For the axiomatic details and a more complete introduction confer to the Appendix of Ref. [Dal82].

In Liouville space, a general linear operator $A$, e.g., the density operator, is represented by a vector. An operator $A$ is rearranged into a vector starting from its ordinary representation as a superposition of all outer products $\left\{\left|u_{m}\right\rangle\left\langle u_{n}\right|\right\}$ in Hilbert space with respect to an orthonormal basis set $\left\{\left|u_{m}\right\rangle\right\}$ of length $N$ :

$$
\begin{aligned}
& \text { Hilbert space: } & A & =\sum_{m, n} A_{m n}\left|u_{m}\right\rangle\left\langle u_{n}\right| \\
& \text { Liouville space: } & |A\rangle\rangle & \left.=\sum_{m, n} A_{m n}\left|u_{m} u_{n}\right\rangle\right\rangle .
\end{aligned}
$$

The Liouville-space ket $\left.\left|u_{m} u_{n}\right\rangle\right\rangle$ used in the above equation can be identified with the operator $\left|u_{m}\right\rangle\left\langle u_{n}\right|$ in Hilbert space. The double bra-ket notation is completely analogous to the ordinary bra-ket notation in Hilbert space and the bra is defined as the Hermitian conjugate $\left\langle\left\langle u_{m} u_{n} \mid=\left(\left|u_{m} u_{n}\right\rangle\right\rangle\right)^{\dagger}\right.$. Most importantly, in Eq. (2.38) one thinks of the matrix elements $A_{m n}$ as a vector of length $N^{2}$. By defining the scalar product of two vectors in Liouville space as the trace

$$
\langle\langle B \mid A\rangle\rangle=\operatorname{Tr}\left(B^{\dagger} A\right)
$$


one finds that $\left\langle\left\langle u_{m} u_{n} \mid A\right\rangle\right\rangle=A_{m n} .^{7}$ In particular, the use of Eq. (2.39) allows us to represent a mixed-state density operator $\rho$ as a vector in Liouville space by

$$
\left.\left.|\rho\rangle\rangle=\sum_{m, n}\left\langle\left\langle u_{m} u_{n} \mid \rho\right\rangle\right\rangle\left|u_{m} u_{n}\right\rangle\right\rangle=\sum_{m, n} \rho_{m n}\left|u_{m} u_{n}\right\rangle\right\rangle
$$

which is formally equivalent to the representation of a pure quantum state in Eq. (2.3). As a next step, it is useful to consider linear operators in Liouville space. Such an operator is defined as

$$
\left.\mathfrak{F}=\sum_{(j, k),(m, n)}\left|u_{j} u_{k}\right\rangle\right\rangle\left\langle\left\langle u_{j} u_{k}|\mathfrak{F}| u_{m} u_{n}\right\rangle\right\rangle\left\langle\left\langle u_{m} u_{n}\right|\right.
$$

and its "matrix elements"

$$
\mathfrak{F}_{j k, m n}=\left\langle\left\langle u_{j} u_{k}|\mathfrak{F}| u_{m} u_{n}\right\rangle\right\rangle
$$

have four indices, but one thinks of them as $N^{2} \times N^{2}$ matrices as suggested by the fusion of indices in Eq. (2.43). The former fact explains why Liouville-space operators are referred to as tetradic operators or superoperators.

In order to render calculations in Liouville space formally identical to calculations of a wave function, the Liouville von-Neumann equation (2.11) has to be brought in a form that is formally isomorphic to the time-dependent Schrödinger equation (2.5). Using Eq. (2.5) and the above Liouville-space notation for a general density operator $|\rho\rangle\rangle$, it is possible to identify a superoperator governing the dynamics in Liouville space. This superoperator is known as the Liouville operator $\mathfrak{L}$. With respect to it, the Liouville von-Neumann equation $(\hbar=1)$

$$
\left.\left.\left.i \frac{d}{d t}|\rho(t)\rangle\right\rangle=|[H, \rho(t)]\rangle\right\rangle=\mathfrak{L}|\rho(t)\rangle\right\rangle .
$$

is formally isomorphic to the time-dependent Schrödinger equation (also see Ref. [Muk95]).

\section{\begin{tabular}{l|l} 
Thermofield dynamics (TFD) & 2.4
\end{tabular}}

Having introduced the representation of a general mixed-state density operator $\rho$ as a vector in Liouville space and its equation of motion (2.44) in this space, one might wonder how a thermal state at temperature $T$ can be obtained in this framework. The answer is provided by thermofield dynamics (TFD) and its very close connection to Liouville space. TFD itself was inspired by the rigorous and axiomatic formulation of quantum field theory at finite temperature. One central realization in this context was that the quantum theory of free fields at $T>0$ can be formulated by doubling the number of degrees of freedom [UHT82].

TFD is a formulation of finite-temperature quantum theory which is suitable for practical calculations in solid state physics [UHT82]. The main concepts such as dynamical response functions and other concepts can be directly translated and applied in the framework of

\footnotetext{
${ }^{7}$ Additionally, in Liouville space the orthonormality conditions $\left\langle\left\langle u_{j} u_{k} \mid u_{m} u_{n}\right\rangle\right\rangle=\delta_{j m} \delta_{k n}$ hold and completeness condition is $\left.\sum_{m, n}\left|u_{m} u_{n}\right\rangle\right\rangle\left\langle\left\langle u_{m} u_{n}\right|=1\right.$.
} 
TFD. In the following, the doubled Hilbert space underlying the TFD description is discussed.

According to Ref. [BD87], each vector $|\rho\rangle\rangle$ in Liouville space can be identified with a pure-state wave function $|\Psi\rangle$ in a doubled Hilbert space $\mathcal{H}_{P} \otimes \mathcal{H}_{Q}$, which is the tensor product space of the physical state space $\mathcal{H}_{P}$ and an auxiliary space $\mathcal{H}_{Q}$ chosen to be isomorphic to $\mathcal{H}_{P}$. Let $\left\{\left|n_{P}\right\rangle\right\}$ respectively $\left\{\left|m_{Q}\right\rangle\right\}$ be a basis set in the physical/auxiliary Hilbert space. Then the basis of the tensor product space is $\left\{\left|n_{P}\right\rangle \otimes\left|m_{Q}\right\rangle\right\}$, which can also be written as $\left\{\left|n_{P} ; m_{Q}\right\rangle\right\}$.

In TFD, a quantum state at inverse temperature $\beta$ is represented by a so-called "statistical state vector" defined in such a doubled Hilbert space $\mathcal{H}_{P} \otimes \mathcal{H}_{Q}$. Originally [Fan57], these statistical states were defined with respect to the eigenbasis of the Hamiltonian $H$ :

$$
|\Psi(\beta)\rangle=\frac{1}{\sqrt{Z(\beta)}} e^{-\beta H / 2}|I\rangle, \quad|I\rangle=\sum_{n}\left|E_{n, P} ; E_{n, Q}\right\rangle .
$$

Alternatively, a statistical state vector is referred to as a thermofield vacuum state. Such a pure-state wave function is also called purification since the formulation using the tensor product space $\mathcal{H}_{P} \otimes \mathcal{H}_{Q}$ purifies general mixed states. With the construction from Eq. (2.45), it can be shown that an ordinary expectation value of a physical observable $A_{P} \otimes I_{Q}$ with respect to $|\Psi(\beta)\rangle$ yields the thermodynamic expectation value at finite temperature:

$$
\begin{aligned}
\left\langle\Psi(\beta)\left|A_{P} \otimes I_{Q}\right| \Psi(\beta)\right\rangle & =\frac{1}{Z(\beta)} \sum_{m, n}\left\langle E_{n, P}\left|e^{-\beta H / 2} A e^{-\beta H / 2}\right| E_{m, P}\right\rangle\left\langle E_{n, Q} \mid E_{m, Q}\right\rangle \\
& =\frac{1}{Z(\beta)} \sum_{m, n}\left\langle E_{n, P}\left|e^{-\beta H / 2} A e^{-\beta H / 2}\right| E_{m, P}\right\rangle \delta_{n, m} \\
& =\frac{1}{Z(\beta)} \sum_{n} e^{-\beta E_{n}}\left\langle E_{n}|A| E_{n}\right\rangle=\operatorname{Tr}(\rho A)=\langle A\rangle .
\end{aligned}
$$

Note that the representation of a thermal state does not depend on the choice of the basis since the state $|I\rangle$ in Eq. (2.45) is invariant for any orthogonal basis $\{|\alpha\rangle\}$ satisfying

$$
\left|E_{n, P}\right\rangle=\sum_{\alpha} U_{n, \alpha}\left|\alpha_{P}\right\rangle \quad \text { and } \quad\left|E_{n, Q}\right\rangle=\sum_{\alpha} U_{n, \alpha}^{*}\left|\alpha_{Q}\right\rangle .
$$

This is known as the general representation theorem of TFD [Suz85] and means that both the state vector and the average do not depend on a particular basis. ${ }^{8}$ One practical relevance of the theorem is, e.g., that the choice of basis can be used to reduce the build-up of entanglement in DMRG computations [KBM12, KBM13, Bar13].

The following remarks are based on Ref. [BD87]. Since it was shown that there exists a Liouville-space vector representing any generalized thermofield state, the thermofield wave function in Eq. (2.45) can also be represented as a vector in Liouville space. Via this identification, the dynamics of the Liouvillian representation of a thermofield state is governed by Eq. (2.44). Thus, the Liouville superoperator becomes the Hamilton operator for the pure-state thermofield wave function. Its action on the physical and auxiliary space is given by

$$
\mathcal{L}=H_{P} \otimes I_{Q}-I_{P} \otimes H_{Q}
$$

\footnotetext{
${ }^{8}$ Thus, TFD can also be applied to non-equilibrium systems [AU85, AU87], which is however beyond the scope of this thesis.
} 
Note that the eigenvalues of the Liouville operator are exactly the differences $\omega_{m, n}=$ $\left(E_{m}-E_{n}\right)$ of the eigenenergies of the Hamiltonian. This is a hint that a Liouvillespace formulation of spectral functions can be rather a natural choice since $\omega_{m, n}$ occur as excitation energies in the Lehmann representation in Eq. (2.27).

In order to stress the close connection to Liouville space, one may say that the dynamics of the pure-state wave function is governed by the Liouville operator $\mathcal{L}{ }^{9}$ Moreover, the pure-state thermofield wave function is usually referred to as purification in the DMRG context (see Sec. 3.6.2), although the term purification is more general and can also be used to refer to arbitrary mixed quantum states and not only thermal states.

A shorter notation typically used in TFD is $H=H_{P} \otimes I_{Q}$ and $\tilde{H}=I_{P} \otimes H_{Q}$. Here the tilde symbol specifies that an operator acts only on the auxiliary space, while it leaves the physical degrees of freedom unchanged. With this the Liouville operator can be written more compactly as $\mathcal{L}=H-\tilde{H}$, which will be used in the next section.

\section{Frequency-space dynamics in Liouville space $\mid 2.5$}

In this section, finite-temperature spectral functions, as defined in Sec. 2.2.2 are reconsidered. Here the goal is to obtain a convenient representation for them which can be employed in practical computations. This can be achieved by a TFD formulation using the Liouville operator $\mathcal{L}$ in Eq. (2.48) above. The following calculation verifies that the expression

$$
S_{A B}(\omega)=\langle\Psi(\beta)|A \delta(\omega-\mathcal{L}) B| \Psi(\beta)\rangle
$$

assumes the general form of the finite-temperature dynamical structure factor in Eq. (2.29). Here one uses the tilde notation for the operators, e.g., $A=\left(A_{P} \otimes I_{Q}\right)$. In a first step, the thermofield wave function $|\Psi(\beta)\rangle$ from Eq. (2.45) is inserted:

$$
\begin{aligned}
S_{A B}(\omega)=\frac{1}{Z(\beta)}\left(\left[\sum_{n^{\prime}}\left\langle E_{n^{\prime}, P}\right|\right.\right. & \left.\left.\otimes\left\langle E_{n^{\prime}, Q}\right|\right] e^{-\beta H / 2}\right) A \delta(\omega-H+\tilde{H}) \times \\
& \times B\left(e^{-\beta H / 2}\left[\sum_{n}\left|E_{n, P}\right\rangle \otimes\left|E_{n, Q}\right\rangle\right]\right) .
\end{aligned}
$$

Then the operators $A, B$, as well as $e^{-\beta H / 2}$ acting on the physical state space are applied to the corresponding eigenstates $\left|E_{n, P}\right\rangle$, both in the left and right square brackets. Moreover, the Hamiltonian $\tilde{H}$, which appears in the delta function, is also applied. This is permissible due to the same arguments leading to Eq. (2.36) above. The corresponding result is

$$
\begin{aligned}
S_{A B}(\omega)= & \frac{1}{Z(\beta)}\left[\sum_{n^{\prime}} e^{-\beta E_{n^{\prime}} / 2}\left(\left\langle E_{n^{\prime}, P}\right| A\right) \otimes\left\langle E_{n^{\prime}, Q}\right|\right] \times \\
& \times\left[\sum_{n} \delta\left(\omega-H+E_{n}\right) e^{-\beta E_{n} / 2}\left(B\left|E_{n, P}\right\rangle\right) \otimes\left|E_{n, Q}\right\rangle\right] .
\end{aligned}
$$

\footnotetext{
${ }^{9}$ In order to distinguish $\mathcal{L}$ from the true Liouville superoperator $\mathfrak{L}$ in this thesis, the symbol is slightly changed.
} 
In the next step, the orthonormality of the eigenstates is exploited to eliminate the double sum after rearranging the above expression with respect to the state spaces $\mathcal{H}_{P}$ and $\mathcal{H}_{Q}$ :

$$
\begin{aligned}
S_{A B}(\omega)= & \frac{1}{Z(\beta)} \sum_{n, n^{\prime}} e^{-\beta E_{n^{\prime}} / 2} e^{-\beta E_{n} / 2} \underbrace{\left\langle E_{n^{\prime}, Q} \mid E_{n, Q}\right\rangle}_{=\delta_{n, n^{\prime}}} \times \\
& \times\left\langle E_{n^{\prime}, P}\left|A \delta\left(\omega-H+E_{n}\right) B\right| E_{n, P}\right\rangle \\
= & \frac{1}{Z(\beta)} \sum_{n} e^{-\beta E_{n}}\left\langle E_{n}\left|A \delta\left(\omega-H+E_{n}\right) B\right| E_{n}\right\rangle .
\end{aligned}
$$

Using the result of the derivation in Eqs. (2.33)-(2.35) for a general eigenstate rather than for the ground state exclusively, one finds

$$
S_{A B}(\omega)=\frac{1}{Z} \sum_{m, n} e^{-\beta E_{n}}\left\langle E_{n}|A| E_{m}\right\rangle\left\langle E_{m}|B| E_{n}\right\rangle \delta\left(\omega-\left(E_{m}-E_{n}\right)\right) .
$$

This implies that the Liouville-space formulation offers a very natural way for the representation of finite-temperature dynamical correlation functions via Eq. (2.49). Concerning the development of numerical methods for the calculation of such finite-temperature quantities in this thesis, the expression will be of practical importance (see Sec. 3.6). 



\section{\begin{tabular}{l|l} 
Computational methods & 3
\end{tabular} \\ Introduction $\mid 3.1$}

The exponential growth of the Hilbert space dimension with system size $L$ makes the computation of static and dynamical quantities - even at $T=0$ - a challenging problem. For instance, a full diagonalization of the Hamiltonian matrix to solve the stationary Schrödinger equation (see Eq. (2.4)) for spin-1/2 systems can only be applied up to roughly 20 spins [NM05, San10]. In order to treat larger quantum systems, more sophisticated methods are necessary. Generally, there is no such numerical method that works for any system. The numerical tools used in this thesis are based on the density-matrix renormalization group (DMRG) which is especially successful for $1 \mathrm{D}$ systems. Originally, DMRG was devised as a numerical method to variationally calculate ground states of strongly correlated quantum many-body systems [Whi92, Whi93], thereby also giving access to local expectation values and static correlation functions. For simple spin Hamiltonians on lattices of a few hundred sites the ground-state energies can determined with an accuracy of up to ten decimal digits for open boundary conditions (OBCs). Instead of working with the full Hilbert space of tremendously high dimensionality, the idea behind DMRG is to work with a much smaller subspace representing the physically relevant portion in the entire Hilbert space. Realizing that the wave function optimized in the DMRG algorithm assumes the form of matrix product states (MPS) [OR95] has led to a reformulation of DMRG in the framework of this powerful variational ansatz class [McC07, Sch11] and a deeper theoretical understanding of the algorithm itself [VCM08]. For 1D Hamiltonians with short-range interactions and a gap in the excitation spectrum, it could be shown that MPS approximate ground-state physics to almost exponential accuracy [VC06]. Therefore, DMRG is considered the most accurate algorithm for 1D systems. The success can be understood by the special quantum mechanical entanglement properties, described by so-called area laws, of the corresponding ground states. These can be captured by MPS descriptions of such states. This illustrates the close connection between DMRG and quantum information theory [ACL12].

After its invention, the method has soon been extended to the computation of zerotemperature spectral functions in the frequency domain [Hal95, KW99, Jec02]. The introduction of DMRG approaches allowing for the time evolution of quantum states [Vid04, WF04, DKSV04, Sch04, MMN05] opened the possibility of evaluating spectral functions also in the time domain followed by subsequent Fourier transforms to frequency and momentum space. In particular for frequency-domain techniques, the MPS formulation turned out to be especially suited for the calculation of excited states and spectral functions $\left[\mathrm{WVS}^{+} 09, \mathrm{HWM}^{+} 11, \mathrm{DWH}^{+} 12\right]$. Therefore, the frequency-domain methods used in this thesis are expressed and implemented in the language of MPS.

In DMRG or MPS methods to compute spectral functions challenges arise due to the necessity to represent quantum states with higher entanglement than in ground states. This 
leads to situations in which these states do not obey an area-law - both in time-domain and frequency-space methods. As a consequence, these approaches have limitations of some kind, e.g., accessible times and hence spectral resolution. Due to increasing computational resources, these zero-temperature approaches have nevertheless fostered significant progress in the study of strongly correlated electron systems (see Sec. 3.5.1).

One main objective of this thesis is the calculation of finite-temperature spectral functions in the frequency domain. However, the MPS ansatz in principle only allows for the representation of pure states. Fortunately, there exist a few ways to address the challenge of mixed-state density operators at finite temperature, which are briefly reviewed at this point. One of the earlier approaches is the DMRG applied to transfer matrices known as TMRG. This could be used to calculate static thermodynamic properties [BXG96, WX97, SK02] as well as dynamical properties at $T>0$ [NWZvdL99, SK05]. The main idea behind this method is the relation between the density matrix of a quantum chain to a partition function of an equivalent two-dimensional classical model. This connection was obtained by means of a Trotter-Suzuki decomposition and an extension of the DMRG to two-dimensional classical systems [Nis95]. A more recent DMRG method is the minimally entangled typically thermal states approach (METTS) [Whi09]. In this, one samples over an ensemble of product states constructed by imaginary-time evolution in order to approximate the finite-temperature state of the system. Since its introduction METTS has been used for the calculation of static thermodynamic quantities [SW10, Alv13] and dynamical correlation functions [BB15, BvDW15]. Until today, most approaches are based on a purification of the mixed density operator. In this thesis, a purification is used to represent thermal states as introduced in Sec. 2.4. In the MPS context, thermal states are obtained by imaginary-time evolution. Spectral functions are then computed via real-time evolution of real-space correlation functions and subsequent Fourier transforms to frequency and/or momentum space [VGRC04, ZV04, FW05, BSW09, KBM12, Bar13, KBM13, PEAT14]. This has been the most common approach to calculate finite-temperature spectral functions so far.

The development of a reliable MPS method for the calculation of finite-temperature spectral functions directly in the frequency domain forms a contrast to the existing timedomain computations using purifications at $T>0$. Devising such an approach is one of the main goals of this work. The envisioned route uses a Liouvillian formulation of the frequency-space dynamics of purifications (see Sec. 2.5) as a foundation in order to extend existing zero-temperature MPS frequency-domain methods to $T>0$.

\section{Structure}

The present chapter provides a step-by-step introduction to the main building blocks needed in order to accomplish this goal. In Sec. 3.2, a brief introduction of the Lanczos method is given since this algorithm will recur as a component of various numerical methods in this chapter. Section 3.3 provides a short summary of the basic properties of MPS which are needed to develop an understanding of the main ideas and concepts behind DMRG. This part also focuses on the numerical aspects that are relevant for this thesis. As DMRG is a state-of-the-art method today, there exist several comprehensive reviews and books covering both the original formulation [PWKH99, Sch05a] and its MPS formulation [McC07, Sch11, Hol12] which is adopted throughout this thesis. Especially Ref. [Sch11] contains many implementation details, e.g., concerning the variational compression of MPS. 
Section 3.4 gives a brief overview on time evolution in the framework of MPS. In particular, the recently developed time evolution via the time-dependent variational principle $\left[\mathrm{HLO}^{+} 14\right]$ is reviewed as it has mainly been used to compute thermal states by imaginarytime evolution in the present work.

In Sec. 3.5, MPS methods for the calculation of zero-temperature spectral functions in the frequency domain are reviewed. This includes continued fraction expansions obtained via the Lanczos algorithm, but the main focus is on Chebyshev expansions. Section 3.5.3 provides the basics of such an expansion in orthogonal polynomials and introduces the kernel polynomial method (KPM) [WWAF06]. In Sec. 3.5.4, the operator-valued Chebyshev expansion of a zero-temperature spectral function in the framework of MPS [HWM ${ }^{+} 11$ ] is reviewed. In this context also recent developments, such as the extrapolation of Chebyshev moments by linear prediction $\left[\mathrm{GTV}^{+} 14\right]$, are addressed.

Finally, the formulation of MPS frequency-domain methods at finite-temperature is presented in Sec. 3.6. The purification of mixed states at $T>0$ is also covered in this context. Then it is shown how the Liouville-space formulation can be exploited to devise expansions in Chebyshev polynomials. A presentation of this approach, which has been newly developed as a part of this work, is contained in Sec. 3.6.4.

\section{\begin{tabular}{l|l} 
The Lanczos method for ground states & 3.2
\end{tabular}}

In contrast to a full diagonalization of the Hamiltonian matrix determining all eigenstates, the Lanczos algorithm [Lan50, NM05] only allows for the calculation of the extremal eigenvalues and the corresponding eigenstates of the Hamiltonian matrix $H$. However, this is sufficient if one is only interested in the ground state and a few low-lying excitations which can be obtained with an accuracy close to machine precision. The Lanczos algorithm belongs to the class of iterative diagonalization techniques [GL96, Saa03]. Therefore, it is possible to treat significantly larger systems than by full diagonalization, e.g., up to $N=50$ spin- $1 / 2$ degrees of freedom.

The basic idea behind the algorithm is to project the Hamiltonian onto a Krylov subspace whose dimension is substantially smaller. Such an $n$-dimensional Krylov subspace is spanned by the following basis

$$
\left\{\left|f_{0}\right\rangle, H\left|f_{0}\right\rangle, H^{2}\left|f_{0}\right\rangle, H^{3}\left|f_{0}\right\rangle, \ldots, H^{n-1}\left|f_{0}\right\rangle\right\}
$$

generated by applying the Hamiltonian to, e.g., a random starting vector $\left|f_{0}\right\rangle .{ }^{1}$ There are several possibilities to construct a basis spanning this subspace. An orthogonal Krylov basis can be obtained via the recursion relation

$$
\begin{aligned}
& \left|f_{i+1}\right\rangle=H\left|f_{i}\right\rangle-a_{i}\left|f_{i}\right\rangle-b_{i}^{2}\left|f_{i-1}\right\rangle \quad\left(b_{0} \equiv 0,\left|f_{-1}\right\rangle \equiv 0\right) \\
& a_{i}=\left\langle f_{i}|H| f_{i}\right\rangle /\left\langle f_{i} \mid f_{i}\right\rangle, \quad b_{i}^{2}=\left\langle f_{i} \mid f_{i}\right\rangle /\left\langle f_{i-1} \mid f_{i-1}\right\rangle .
\end{aligned}
$$

The $\left|f_{i}\right\rangle$ are referred to as Lanczos vectors since the quantum states are usually encoded as ordinary vectors. The key operation in the Lanczos recursion is to apply the Hamiltonian. A random starting vector $\left|f_{0}\right\rangle$ is used as it most probably has a finite overlap with the

\footnotetext{
${ }^{1}$ This basis is generated by the power method which can also be used to motivate the Lanczos algorithm as a projection method, see Ref. [NM05].
} 
ground state $\left|E_{0}\right\rangle$. After $n$ iterations the Hamiltonian is approximated by a tridiagonal matrix $T_{n}$ represented in the basis of the normalized Lanczos vectors $\left|f_{i}^{\prime}\right\rangle$

$$
T_{n}=\left(\begin{array}{cccc}
a_{0} & b_{1} & & 0 \\
b_{1} & a_{1} & \ddots & \\
& \ddots & \ddots & b_{n-1} \\
0 & & b_{n-1} & a_{n-1}
\end{array}\right) .
$$

The lowest eigenvalues and eigenstates given by the diagonalization of this matrix very well approximate ${ }^{2}$ those of the full Hamiltonian since the best convergence is obtained for the extremal eigenvalues. Note that a tridiagonal matrix can be diagonalized very efficiently with a computational cost of $\mathcal{O}\left(n^{2}\right)$. As the eigenstates are obtained in the Lanczos basis they still need to be expanded with respect to the many-body basis, e.g., by running the algorithm again.

One problem of the algorithm is the partial loss of orthogonality of the $\left|f_{i}\right\rangle$ due to numerical errors. This leads to so-called ghosts in the energy convergence scheme. These are already converged eigenvalues which, after a certain number of iterations, spontaneously collapse onto lower levels of the spectrum. The result is an unphysical multiplicity of some eigenvalues. Important technical details of the method as well as possible solutions of this problem, such as a reorthogonalization of the Lanczos vectors, are discussed in Refs. [GL96, CW02, NM05, Dar12].

The Lanczos method is presented at the beginning of the present chapter, since it is used as a key component of various DMRG methods in this thesis. These applications go beyond the calculation of ground states for which the method was initially devised. For instance, iterative eigensolvers such as the Lanczos algorithm represent an important component of the ground-state DMRG algorithm (see Sec.3.3.3). Moreover, the method can also be used in time-evolution methods as it can be employed to approximate exponentials of the form $\mathrm{e}^{-i H \Delta t}$ (see for instance Sec. 3.4.3). Furthermore, the Lanczos algorithm has an extension allowing for the calculation of dynamical properties via a continued fraction expansion (see Sec. 3.5.2). All of these applications are used in this work, with the latter being most important due to the topic of this thesis.

\section{Matrix product states and the density-matrix renormalization group}

\section{Entanglement}

Decisive progress in understanding the success of DMRG and MPS has been made with concepts from quantum information theory such as the entanglement entropy [FSW08]. The subjects discussed here are hence not only relevant in the DMRG context as they are also important concerning the general characterization of quantum states. In the quantum many-particle problems studied in this work, the individual parts of the system interact with each other. Therefore, the quantum state of the single components cannot be considered independently of each other and the corresponding degrees of freedom

\footnotetext{
${ }^{2}$ Even after only $n \sim 100$ iterations for the ground state.
} 


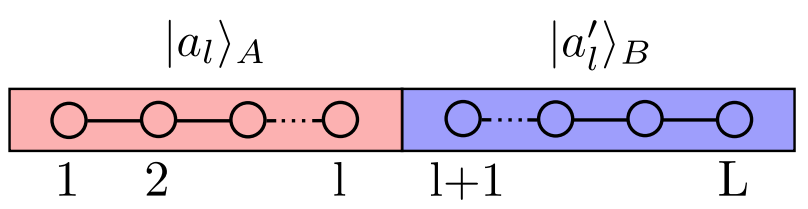

Figure 3.1: Bipartition of a 1D lattice with OBCs into blocks $A$ (sites 1 to $l$ ) and $B$ (sites $l+1$ to $L)$. Each block has an orthonormal basis $\left\{\left|a_{l}\right\rangle_{A}\right\}\left(\left\{\left|a_{l}^{\prime}\right\rangle_{B}\right\}\right)$ of length $N_{A}\left(N_{B}\right)$ that is used for a tensor-product representation of the wave function $|\Psi\rangle$ describing the state of the entire combined system in Eq. (3.3).

are intertwined. In such situations, one speaks of entangled states or the presence of entanglement. In the following paragraphs, the focus will be on the connection between reduced density matrices and quantum mechanical entanglement which is crucial for the success of DMRG and MPS.

\section{Reduced density matrices}

Let us first consider a wave function $|\Psi\rangle$ with respect to a bipartition of the system as, e.g., depicted in Fig. 3.1 for a 1D lattice. The Hilbert spaces of the two subsystems $A$ and $B$ may each have an orthonormal basis $\left\{\left|a_{l}\right\rangle_{A}\right\}$ (or $\left\{\left|a_{l}^{\prime}\right\rangle_{B}\right\}$ ) of length $N_{A}\left(N_{B}\right)$. Then the wave function $|\Psi\rangle$ of the composite system can be represented by

$$
|\Psi\rangle=\sum_{a_{l}, a_{l}^{\prime}} \Psi_{a_{l}, a_{l}^{\prime}}\left|a_{l}\right\rangle_{A}\left|a_{l}^{\prime}\right\rangle_{B}
$$

Any rectangular matrix $\Psi$ can be decomposed by a singular value decomposition (SVD) yielding

$$
\Psi=U S V^{\dagger}
$$

Here the matrix $U$ has orthonormal columns, $S$ is a diagonal matrix, and the rows of $V$ are orthonormal. The diagonal elements $S_{a a}=s_{a}$ of $S$ are non-negative and are called singular values. Defining a new basis on subsystem $A$ by $\left\{|a\rangle_{A}=\sum_{a_{l}} U_{a_{l}, a}\left|a_{l}\right\rangle_{A}\right\}$ and similarly for subsystem $B$ by $\left\{|a\rangle_{B}=\sum_{a_{l}^{\prime}} V_{a_{l}^{\prime}, a}^{*}\left|a_{l}^{\prime}\right\rangle_{B}\right\}$, one can formulate the Schmidt decomposition as

$$
|\Psi\rangle=\sum_{a=1}^{\min \left(N_{A}, N_{B}\right)} s_{a}|a\rangle_{A}|a\rangle_{B} .
$$

It is important that the single sum only runs up to the Hilbert space dimension of the smaller subspace. The entanglement properties of a state $|\Psi\rangle$ are contained in the Schmidt coefficients $s_{a}$. For instance, if only one single coefficient $s_{a}$ is nonvanishing, $|\Psi\rangle$ is a product state. The state is called maximally entangled, if all values $s_{a}$ are of equal magnitude. If $|\Psi\rangle$ is normalized, one finds $\sum_{a} s_{a}^{2}=1$.

Similarly, one may also consider the density matrix $\rho=|\Psi\rangle\langle\Psi|$ and trace out the degrees of freedom belonging to one subsystem. This yields the reduced density matrix of the other subsystem

$$
\rho_{A}=\operatorname{Tr}_{B} \rho \quad \text { or } \quad \rho_{B}=\operatorname{Tr}_{A} \rho .
$$

Using Eq. (3.5), the diagonal forms of the reduced density matrices are given by

$$
\rho_{\alpha}=\sum_{n}\left|s_{a}\right|^{2}|a\rangle_{\alpha \alpha}\langle a|, \quad \alpha=A, B
$$


This reveals that both $\rho_{A}$ and $\rho_{B}$ possess the same nonvanishing eigenvalues $w_{a}=\left|s_{a}\right|^{2}$ and $|a\rangle_{\alpha}$ are the eigenstates of $\rho_{\alpha}$. This eigenspectrum encodes the entanglement properties of the state with respect to a bipartition. The selection of an optimal subspace of the total Hilbert space, e.g., in the course of a DMRG ground-state search, basically proceeds by retaining only the $m$ states $|a\rangle_{\alpha}$ with the largest eigenvalues $w_{a}$, whereas the other states with smaller weights are discarded. This truncation of the Hilbert space is motivated by the Schmidt decomposition (3.5), but is only successful if the discarded weight is negligibly small. Therefore, the decay of the eigenvalue spectra of the reduced density-matrix for each bipartition is crucial for the success of DMRG and MPS methods. ${ }^{3}$

\section{Entanglement entropy of ground states}

Quantifying and comparing entangled states via the spectra of reduced density matrices offers a full description of the entanglement content, but the use of a simple measure related to the eigenvalues $w_{a}$ would be a lot more convenient in many cases. Such a measure is the von Neumann entropy ${ }^{4}$ which is also called entanglement entropy. It quantifies the entanglement between to parts $A$ and $B$ of the system:

$$
S(A)=-\operatorname{Tr}\left(\rho_{A} \log _{2} \rho_{A}\right)=-\sum_{a} w_{a} \log _{2} w_{a}
$$

From this definition, it is clear that $S(A)=S(B)$ and the von Neumann entropy vanishes for product states and assumes its maximal value $S=\log _{2}(w)$ in a maximally entangled state, where $w_{a}=w$ for all $a$.

There have been many works studying the entanglement entropy of ground states of Hamiltonians with short-range interactions, oftentimes based on conformal field theories [Sre93, CC04, PEDC05] or spin Hamiltonians [VLRK03, BHV06, EO06]. These studies found a universal scaling behavior of the entanglement entropy: the entanglement entropy of ground states of Hamiltonians with short-range interactions was found to scale with the surface area, i.e., the boundary between the two subsystems. Consequently, these scaling dependencies are called boundary or area laws and depend on the spatial dimension $D$ of the system under consideration. Note that the subsequent presentation of entanglement properties is not comprehensive. Good reviews on this topic are given in Refs. [AFOV08, ECP10, EV11, ACL12].

The entanglement entropy between a block $A$ consisting of $l^{D}$ sites and the rest of the system scales as the size of its boundary:

$$
S(A) \propto l^{D-1} .
$$

Strictly speaking, this result is only valid for gapped Hamiltonians with short-range interactions. Such systems are referred to as non-critical. For critical systems which are gapless, the area laws are modified by logarithmic corrections

$$
S(A) \propto l^{D-1} \log _{2}(l) .
$$

These results have been obtained in the thermodynamic limit. For $l \rightarrow \infty, D=1$ and in the non-critical case, the entanglement entropy is proportional to a constant. However,

\footnotetext{
${ }^{3} \mathrm{~A}$ more detailed discussion of the conceptual background of the DMRG and, in particular, the decay of the spectrum of reduced density matrices in ground states can be found in Chapter 20 of Ref. [FSW08].

${ }^{4}$ The von Neumann entropy corresponds to the Shannon entropy in information theory [ACL12].
} 
for finite systems with open boundary conditions (OBCs) in one dimension $D=1$, a setup frequently encountered in DMRG calculations, the entanglement entropy only approaches this constant for block sizes larger than the correlation length. A good representation or approximation of ground states or other quantum states by an MPS depends on whether MPS can encode the entanglement properties inherent to the state of interest. Switching to a theoretical perspective, in the more favorable case of 1D systems with short-range interactions and a gap, it could be proven that the entanglement entropy of an MPS with fixed bond dimension remains constant upon increasing the system size [Has07a, Has07b]. It is said that MPS represent ground-state physics faithfully, i.e., approximate it up to almost exponential accuracy [VC06].

For critical systems the logarithmic correction of a subsystem of length $l$ in one dimension was determined as

$$
S(A) \approx \frac{c}{3} \log _{2}(l)+g
$$

where $c$ denotes the central charge of the related conformal field theory and $g$ is a constant [VLRK03]. From a rigid point of view, ground states of critical systems can therefore in general not be represented faithfully by MPS. However, even in the critical case, it is possible to accurately approximate the ground-state properties up to some reasonably large length scale [VCM08]. The price to be paid is an increase in the maximal matrix dimension $m$ used to represent an MPS which will be introduced in the following section. The computational cost usually only scales as $\mathcal{O}\left(\mathrm{m}^{3}\right)$ for MPS methods.

\section{Formalism of matrix product states (MPS)}

\section{Matrix product states}

The following passages briefly review matrix product sates (MPS). First of all, consider a general pure quantum state, expanded in the tensor product basis

$$
|\mathbf{s}\rangle=\left|s_{1}, s_{2}, \ldots, s_{L}\right\rangle=\left|s_{1}\right\rangle \otimes\left|s_{2}\right\rangle \otimes \cdots \otimes\left|s_{L}\right\rangle
$$

of the $d$-dimensional local bases $\left\{\left|s_{l}\right\rangle ; s_{l}=1, \ldots, d\right\}$ at site $l$, on a chain of length $L$

$$
|\Psi\rangle=\sum_{s_{1}, \ldots, s_{L}} c_{s_{1} \ldots s_{L}}|\mathbf{s}\rangle
$$

The coefficients $c_{s_{1} \ldots s_{L}}$ can be written as a trace over a product of matrices $M^{s_{l}}$ :

$$
c_{s_{1} \ldots s_{L}}=\langle\mathbf{s} \mid \Psi\rangle=\operatorname{Tr} M^{s_{1}} M^{s_{2}} \cdots M^{s_{L}} .
$$

As the matrix index $s_{l}$ suggests, there are $d$ matrices at each site used to represent an MPS on the entire lattice. Such a local set of matrices may just as well be considered as a rank-3 tensor $M_{a_{l-1}, a_{l}}^{s_{l}}$. Here $s_{l}$ is a physical index and both $a_{l-1}$ and $a_{l}$ are referred to as internal indices of the MPS matrices. The notion of locality is inherent to MPS and also essential for algorithms formulated in the framework of MPS since it allows for local updates. Instead of working with a huge number of coefficients growing exponentially with system size, these algorithms only use a number of matrix elements that is increasing polynomially with $L$ and hence numerically manageable. Therefore, the matrices in Eq. (3.14) have to 
be truncated to circumvent the problem of exponentially growing matrix dimensions. In the case of OBCs which is adopted for the DMRG calculations in this work, one treats $M^{s_{1}}$ and $M^{s_{L}}$ as boundary vectors. ${ }^{5}$ Then the truncated MPS consists of matrices whose dimensions go as $(1 \times d),\left(d \times d^{2}\right), \ldots,\left(d^{n} \times m\right),(m \times m), \ldots,(m \times m),\left(m \times d^{n}\right), \ldots,\left(d^{2} \times d\right)$, $(d \times 1)$. Here $m$ denotes the maximal matrix dimension that is fixed and also referred to as internal bond dimension of the MPS. Such a truncation can be achieved in the spirit of a Schmidt decomposition using a singular value decomposition (SVD) compression (see Sec. 3.3.1). To this end, one splits the system into two parts $A$ and $B$ and wants to obtain orthonormal bases on both subsystems. The construction of these bases is significantly simplified if the matrices fulfill normalization conditions. Therefore, we call the matrices $A^{s_{l}}\left(B^{s_{l}}\right)$ at a specific site left-normalized respectively right-normalized if they obey

$$
\sum_{s_{i}} A^{s_{i} \dagger} A^{s_{i}}=I \quad \text { or } \quad \sum_{s_{i}} B^{s_{i}} B^{s_{i} \dagger}=I
$$

One may also say left-canonical instead of left-normalized. Unnormalized matrices are denoted by $M^{s_{l}}$ in this notation. The orthonormal basis on each block are then obtained as

$$
\begin{aligned}
\left|\Phi_{a_{l}}^{[1: l]}\right\rangle_{A} & =\left(A^{s_{1}} A^{s_{2}} \ldots A^{s_{l}}\right)_{1, a_{l}}\left|s_{1}, s_{2}, \ldots, s_{l}\right\rangle, \\
\left|\Phi_{a_{l}}^{[l+1: L]}\right\rangle_{B} & =\left(A^{s_{l+1}} A^{s_{l+2}} \ldots A^{s_{L}}\right)_{a_{l}, 1}\left|s_{l+1}, s_{l+2}, \ldots, s_{L}\right\rangle .
\end{aligned}
$$

Then the Schmidt decomposition of an MPS can be written as

$$
|\Psi\rangle=\sum_{a_{l}} s_{a_{l}}\left|\Phi_{a_{l}}^{[1: l]}\right\rangle_{A}\left|\Phi_{a_{l}}^{[l+1: L]}\right\rangle_{B}
$$

In order to treat the degrees of freedom at site $l$ individually, which is for instance important for updates in a ground-state search with MPS, one may partition the chain into three parts: a block left to this site, the individual site, and a block right to it. This yields the so-called mixed-canonical representation of an MPS

$$
|\Psi\rangle=\sum_{a_{l-1}, a_{l}, s_{l}} M_{a_{l-1}, a_{l}}^{s_{l}}\left|\Phi_{a_{l-1}}^{[1: l-1]}\right\rangle_{A}\left|s_{l}\right\rangle\left|\Phi_{a_{l}}^{[l+1: L]}\right\rangle_{B}
$$

where the matrices in block $A(B)$ are left-normalized (right-normalized) resulting in orthonormal bases for these parts of the partition. The corresponding tripartition of the lattice is illustrated in Fig. 3.2. It it possible to conveniently move the local site $l$ in such a mixed-canonical representation to site $l+1$.

Technically, this is achieved by merging two indices ${ }^{6}$ of the rank-3 tensor $M_{a_{l-1}, a_{l}}^{s_{l}}$ yielding the matrix $M_{\left(s_{l}, a_{l-1}\right), a_{l}}$ which can be decomposed by an SVD

$$
M_{\left(s_{l}, a_{l-1}\right), a_{l}}=U S V^{\dagger}
$$

To undo the merging operation, the matrix $U$ satisfying $U^{\dagger} U=I$ can be split into the set of left-normalized matrices $A^{s_{l}}$. The matrix $S V^{\dagger}$ is multiplied to the matrices $B^{s_{i+1}}$ in order to obtain $M^{s_{i+1}}$. In fact, if one only wants to change the normalization

\footnotetext{
${ }^{5}$ Then the trace can be omitted in Eq. (3.14). However, it is important for periodic boundary conditions.

${ }^{6}$ Ref. [SPV11] gives a good review discussing such operations in general tensor network states.
} 


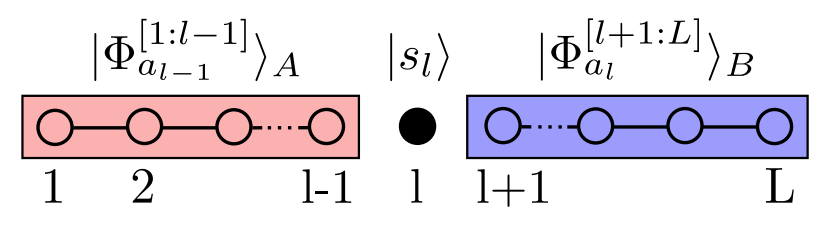

Figure 3.2: Tripartition of a 1D lattice into block $A$ (sites 1 to $l-1$ ), one single site that is treated exactly, and block $B$ (sites $l+1$ to $L$ ). For each block the basis set is specified. Note that the block bases $\left|\Phi_{a_{l-1}}^{[1: l-1]}\right\rangle_{A}$ and $\left|\Phi_{a_{l}}^{[l+1: L]}\right\rangle_{B}$ have to be orthonormal in order to arrive at a mixed-canonical representation (3.19) and exploit its benefits.

of matrices without truncating them, it is not necessary to perform an SVD. Instead a computationally cheaper QR decomposition [Sch11] is favored in this thesis and an SVD is only performed whenever the singular values are needed, e.g., in order to assess the quality of a compression, or if a calculation of the entanglement entropy is desired (see Eq. (3.8)).

Moreover, the updated $A^{s_{l}}$ can be used to iteratively construct the orthonormal basis of the block consisting of sites 1 through $l$ by the relation

$$
\left|\Phi_{a_{l+1}}^{[1: l+1]}\right\rangle_{A}=\sum_{a_{l}, s_{l+1}} A_{a_{l}, a_{l+1}}^{s_{l+1}}\left|\Phi_{a_{l}}^{[1: l]}\right\rangle_{A}\left|s_{l+1}\right\rangle
$$

In DMRG-based algorithms, this growth/shrinkage procedure is used to increase block $A$ by a single site, which is removed from block $B$, until $A$ is of length $L-1$. Then the roles of the two blocks in this growth procedure are reversed until convergence is reached. In fact, also a right-to-left sweep is possible and proceeds in a similar manner as the individual steps of a left-to-right sweep explained above. For details on how to absorb a site $l$ into the right block confer to Ref. [Sch11]. For the case of an SVD compression at each bond in such a sweep, it could be shown that the cumulated error of the compressed state with reduced internal matrix dimensions $\left|\Psi_{\text {compr }}\right\rangle$ has the following bound with respect to the 2-norm:

$$
\||\Psi\rangle-\left|\Psi_{\text {compr }}\right\rangle \|_{2}^{2} \leq 2 \sum_{l} \epsilon_{l}(D)
$$

where $\epsilon_{l}(D)=\sum_{j>m} s_{j}^{2}$ is the sum of the neglected squared Schmidt coefficients at bond $l$, also known as discarded weight.

Furthermore, it has to be noted that the choice of left or right-canonical matrices does by far not exhaust the gauge degrees of freedom of an MPS. This choice rather represents a convenient special case. There are even more gauge transforms leaving the physical state unchanged. This can be understood by the following example. One may insert an invertible matrix $X$ between any two adjacent matrices

$$
M^{s_{l}} \rightarrow M^{s_{l}} X, \quad M^{s_{l+1}} \rightarrow X^{-1} M^{s_{l+1}} .
$$

Although the MPS matrices have changed, the physical content remains the same.

\section{Calculation of overlaps}

Given the two MPS $|\tilde{\Psi}\rangle$ and $|\Psi\rangle$, their overlap can be written as

$$
\langle\tilde{\Psi} \mid \Psi\rangle=\sum_{s_{1}, \ldots, s_{L}} \tilde{M}^{s_{1} *} \cdots \tilde{M}^{s_{L} *} M^{s_{1}} \cdots M^{s_{L}},
$$


where the $\tilde{M}^{s_{l} *}$ are the complex conjugates of the MPS matrices of $|\tilde{\Psi}\rangle$. For OBCs the matrix product $\tilde{M}^{s_{1}} \ldots \tilde{M}^{s_{L}}$ is a scalar which can be transposed leading to a reversed ordering

$$
\langle\tilde{\Psi} \mid \Psi\rangle=\sum_{s_{1}, \ldots, s_{L}} \tilde{M}^{s_{L} \dagger} \cdots \tilde{M}^{s_{1} \dagger} M^{s_{1}} \cdots M^{s_{L}}
$$

In the expression there are so-called contractions both over the matrix indices being concealed in the matrix multiplications and over the physical indices $\left(s_{1}, \ldots, s_{L}\right)$. This example is supposed to convey the importance of contracting the indices in an optimal order. It is a general issue of MPS calculations and not merely encountered in the calculation of overlaps. Contracting the internal matrix indices in the first place and subsequently treating the physical indices would amount to a summation of $d^{L}$ terms growing exponentially with system size. However, rearranging the summation in Eq. (3.25) gives

$$
\langle\tilde{\Psi} \mid \Psi\rangle=\sum_{s_{L}} \tilde{M}^{s_{L} \dagger}\left(\ldots\left(\sum_{s_{2}} \tilde{M}^{s_{2} \dagger}\left(\sum_{s_{1}} \tilde{M}^{s_{1} \dagger} M^{s_{1}}\right) M^{s_{2}}\right) \cdots\right) M^{s_{L}} .
$$

The above expression is contracted starting from the inside. First, the vectors $\tilde{M}^{s_{1} \dagger}$ and $M^{s_{1}}$ are multiplied ( $d \mathrm{~m}^{3}$ operations). The results for different states $s_{1}$ are added. ${ }^{7}$ Then the matrices $\tilde{M}^{s_{2} \dagger}$ and $M^{s_{2}}$ are contracted with the innermost result in between and added afterwards. This iterative procedure is continued until the outermost sum over $s_{L}$ is performed. Therefore, the complexity scales polynomially with on the order of $\left(\mathrm{Ldm}^{3}\right)$ operations. The procedure is referred to as optimal bracketing and is very important in order to efficiently contract matrices in the MPS framework. Related examples of such advantageous bracketing occur in many situations, e.g., the application of a Hamiltonian matrix product operator (see below) to a mixed canonical state. Further detailed examples are given in Ref. [Sch11].

A useful insight concerning an MPS $|\Psi\rangle$ consisting only of left-normalized matrices is that it is normalized, i.e., $\langle\Psi \mid \Psi\rangle=1$. The result is obtained by inserting the left-normalization condition from Eq. (3.15) into Eq. (3.26). The normalization also applies to MPS consisting of right-normalized matrices.

\section{Addition of MPS}

An operation frequently needed due to the recursive character of the frequency-domain methods (see, e.g., Eq. (3.93)) mainly used in this thesis is the addition of MPS. Here two unnormalized MPS $|\Psi\rangle$ and $|\tilde{\Psi}\rangle$ are considered. Their sum is given by

$$
|\Psi\rangle+|\tilde{\Psi}\rangle=\sum_{s_{1}, \ldots, s_{L}} \operatorname{Tr} N^{s_{1}} N^{s_{2}} \cdots N^{s_{L}}|\mathbf{s}\rangle, \quad N^{s_{l}}=M^{s_{l}} \oplus \tilde{M}^{s_{l}} .
$$

The symbol $\oplus$ signifies that $N^{s_{l}}$ is a block diagonal matrix with two diagonal blocks $M^{s_{l}}$ and $\tilde{M}^{s_{l}}$. Thus, the internal bond dimensions of the MPS matrices add under addition and MPS of a given dimension are not closed under this operation. For OBCs, it is necessary to treat the matrices at the first and the last sites differently to conserve their boundaryvector shape. So a row $\left[M^{s_{1}}, \tilde{M}^{s_{1}}\right]$ respectively column vector $\left[M^{s_{L}}, \tilde{M}^{s_{L}}\right]^{T}$ is formed [Sch11]. In order to maintain an MPS with computationally manageable dimensions, it is oftentimes practical to compress sums of matrix product states (see Sec. 3.3.4 below).

\footnotetext{
${ }^{7}$ These first two steps as well as further contractions in Eq. (3.26) can be efficiently implemented using the dgemm or zgemm routines of BLAS libraries. The routines deal with matrix expressions of the form $C \leftarrow \alpha A \cdot B+\beta C$, where $\alpha$ and $\beta$ are scalars.
} 


\section{Matrix product operators (MPO)}

In the framework of MPS, it is natural to express operator matrix elements in analogy to Eq. (3.14) as

$$
\left\langle\mathbf{s}|A| \mathbf{s}^{\prime}\right\rangle=W^{s_{1}, s_{1}^{\prime}} W^{s_{2}, s_{2}^{\prime}} \ldots W^{s_{L-1}, s_{L-1}^{\prime}} W^{s_{L}, s_{L}^{\prime}}|\mathbf{s}\rangle\left\langle\mathbf{s}^{\prime}\right|
$$

The notion of locality is preserved since at each site there is a rank-4 tensor $W_{b_{l-1}, b_{l}}^{s_{l}, s_{l}^{\prime}}$ with two physical indices denoting the physical state at that site before $s_{l}$ and after $s_{l}^{\prime}$ the application of the operator. ${ }^{8}$ Therefore, the matrix product operator (MPO) representation of an operator $A$ is given by

$$
A=\sum_{\mathbf{s}, \mathbf{s}^{\prime}} W^{s_{1}, s_{1}^{\prime}} W^{s_{2}, s_{2}^{\prime}} \cdots W^{s_{L-1}, s_{L-1}^{\prime}} W^{s_{L}, s_{L}^{\prime}}|\mathbf{s}\rangle\left\langle\mathbf{s}^{\prime}\right|
$$

Note that any operator can be represented as an MPO. For OBCs the outermost MPO matrices also serve as boundary vectors, which is in complete analogy to MPS. In this thesis, mostly short-range Heisenberg Hamiltonians are expressed in this manner. ${ }^{9}$ As an example, the internal MPO dimensions (associated indices $b_{l-1}$ and $b_{l}$ ) for an $X X Z$ Heisenberg chain with nearest-neighbor couplings have a dimension of $D=5$. Therefore, such operators can be stated and applied exactly without the need for truncation of the MPO matrices. Applying an MPO to an MPS leads to an MPS with increased internal bond dimensions, which can be inferred from the updated matrices in

$$
|\tilde{\Psi}\rangle=A|\Psi\rangle=\sum_{\mathbf{s}} \tilde{M}^{s_{1}} \tilde{M}^{s_{2}} \cdots \tilde{M}^{s_{L}}|\mathbf{s}\rangle, \quad \tilde{M}^{s_{l}}=\sum_{s_{l}^{\prime}} W_{b_{l-1}, b_{l}}^{s_{l} s_{l}^{\prime}} M_{a_{l-1}, a_{l}}^{s_{l}^{\prime}} .
$$

The dimension of the resulting matrices $\tilde{M}^{s_{l}}$ is given as the product $(m D)$, where $D$ denotes the internal bond dimension of the MPO and $m$ the one of the MPS. An important implication arising from this is that in each step of, e.g., the MPS-based Lanczos recursion (see Eq. (3.2)) the bond dimensions multiply leading to rapid increase. Hence, there is the need of compressing the updated state after each such iteration if one applies the Hamiltonian according to Eq. (3.30). Besides an SVD compression, there is also a variational compression scheme reviewed in Sec. 3.3.4 below.

\section{Applying an MPO to a mixed-canonical MPS}

Instead of applying an MPO globally, as in Eq. (3.30), there also exist other possibilities. In this context, globally means involving operations at each site which are generally not exploiting the normalization of the state. It is sometimes more convenient to locally apply an MPO such as the Hamiltonian to an MPS in a mixed canonical representation, see Eq. (3.19). To this end, it is necessary to construct tensors $L_{b_{l-1}, a_{l-1}^{\prime}}^{a_{l-1}}$ and $R_{b_{l}}^{a_{l}, a_{l}^{\prime}}$ that include the action of the MPO Hamiltonian on the left respectively right block with respect to the current site $l$. In the growth/shrinkage procedure of the blocks during a left-to-right sweep, the $L$ tensors can be updated iteratively by an efficient recursion, ${ }^{10}$ while the $R$ tensors that were calculated in the previous right-to-left sweep can be reused for the

\footnotetext{
${ }^{8}$ Here OBCs are assumed. Therefore, the trace is omitted as the sets of matrices $W^{s_{1}}, s_{1}^{\prime}$ and $W^{s_{L}, s_{L}^{\prime}}$ serve as boundary vectors similar to the MPS convention for OBCs.

${ }^{9}$ For details on MPO representations of Hamiltonians confer to Sec. 6.1 of Ref. [Sch11].

${ }^{10}$ See, e.g., Eq. (195) in Ref. [Sch11].
} 
block of decreasing size. With these tensors the Hamiltonian can be applied to a mixed canonical MPS as

$$
H|\Psi\rangle=\sum_{b_{l-1}, a_{l-1}^{\prime}} L_{b_{l-1}}^{a_{l-1}, a_{l-1}^{\prime}}\left(\sum_{b_{l} s_{l}^{\prime}} W_{b_{l-1}, b_{l}}^{s_{l}, s_{l}^{\prime}}\left(\sum_{a_{l}^{\prime}} R_{b_{l}}^{a_{l}, a_{l}^{\prime}} M_{a_{l-1}^{\prime}, a_{l}^{\prime}}^{s_{l}^{\prime}}\right)\right)\left|\Phi_{a_{l-1}}^{[1: l-1]}\right\rangle_{A}\left|s_{l}\right\rangle\left|\Phi_{a_{l}}^{[l+1: L]}\right\rangle_{B} .
$$

For the sake of efficiency, one should again start with the operation in the innermost parenthesis and perform the outermost sum at the end of these contractions. Equation (3.31) contains the key operation of the ground-state search algorithm presented in the next section.

\section{Ground-state search}

The calculation of the ground state $\left|E_{0}\right\rangle$ and its energy $E_{0}$ can be formulated as a minimization problem, in which the energy $E$

$$
E_{0} \leq E=\min _{|\Psi\rangle} \frac{\langle\Psi|H| \Psi\rangle}{\langle\Psi \mid \Psi\rangle}
$$

is minimized by optimizing the parameters of the wave function $|\Psi\rangle$. Equation (3.32) is known as the (time-independent) variational principle in quantum mechanics. The variational ansatz class used here are MPS of finite bond dimensions. Therefore, the DMRG algorithm - originally invented as a method to compute ground-state properties [Whi92, Whi93] - is regarded as an application of the time-independent variational principle to MPS as the underlying variational ansatz class [VCM08]. The minimization problem in Eq. (3.32) can be treated by using the Lagrange formalism, i.e., $\langle\Psi|H| \Psi\rangle$ is minimized under the constraint that $\langle\Psi \mid \Psi\rangle$ remains normalized. The energy to be minimized takes the role of the Lagrange multiplier $\lambda$ in the following Lagrange function:

$$
L=\langle\Psi|H| \Psi\rangle-\lambda(\langle\Psi \mid \Psi\rangle-1) .
$$

Even for an MPS with finite internal bond dimensions denoted by $m$, there are roughly $\left(L d m^{2}\right)$ optimization parameters. Thus, the usual procedure of differentiation with respect to each of these parameters yielding a set of coupled equations can only be successful for rather small systems and dimensions $m$. Otherwise it is not tractable to globally optimize these parameters. At this point the mixed-canonical representation of an MPS is advantageous, since it allows for optimization of the rank-3 tensor $M_{a_{l-1}, a_{l}}^{s_{l}}$ at a distinguished site. Therefore, one may formally differentiate only with respect to these parameters. Setting the resulting equations to zero yields

$$
0 \stackrel{!}{=} \frac{\partial L}{\partial M_{a_{l-1}, a_{l}}^{s_{l}}}=\frac{\partial}{\partial M_{a_{l-1}, a_{l}}^{s_{l}}}(\langle\Psi|H| \Psi\rangle)-\lambda \frac{\partial}{\partial M_{a_{l-1}, a_{l}}^{s_{l}}}(\langle\Psi \mid \Psi\rangle) .
$$

It can be shown [Sch11] that for OBCs and an MPS in the mixed-canonical representation Equation (3.34) reduces to an ordinary eigenvalue problem for the local matrix parameters $M_{a_{l-1}, a_{l}}^{s_{l}}$ after merging their indices to obtain the vector $\nu_{\left(s_{l}, a_{l-1}, a_{l}\right)}$ :

$$
H^{\mathrm{eff}} \nu-\lambda \nu=0 .
$$


Here $H^{\mathrm{eff}}$ is a $\left(d m^{2} \times d m^{2}\right)$ matrix

$$
H_{\left(s_{l} a_{l-1} a_{l}\right),\left(s_{l}^{\prime} a_{l-1}^{\prime} a_{l}^{\prime}\right)}^{\mathrm{eff}}=\sum_{b_{l-1}, b_{l}} L_{b_{l-1}}^{a_{l-1}, a_{l-1}^{\prime}} W_{b_{l-1}, b_{l}}^{s_{l}, s_{l}^{\prime}} R_{b_{l}}^{a_{l}, a_{l}^{\prime}}
$$

which can be fully diagonalized for small systems. However, in a ground-state search one only needs to determine the ground-state of this matrix. This can be achieved by an iterative eigensolver [Saa03] such as the Lanczos algorithm (see Sec. 3.2) which is used in this thesis. ${ }^{11}$ To speed up convergence significantly, one uses the current local parameters $\nu_{\left(s_{l}, a_{l-1}, a_{l}\right)}$ as the starting vector. The resulting vector obtained by the iterative eigensolver is then reshaped to update $M_{a_{l-1}, a_{l}}^{s_{l}}$. As a next step, the current site with unnormalized matrices is moved to the left or right depending on the current direction of sweeping, while the mixed-canonical representation is maintained. In contrast to this single-site algorithm for finite systems, the traditional finite-system DMRG [Whi92, Whi93] has been formulated as a two-site variant. This means that the system is partitioned in a right and a left block with two adjacent single sites in between. Such a two-site algorithm is less prone to getting stuck in a non-global energy minimum since its ansatz space is slightly enlarged in comparison to the single-site variant. This is a generic problem of variational ground-state searches: even if the variationally determined ground-state energy is close to the true one, the ground-state properties such as correlation functions may differ from the true ground-state. ${ }^{12}$ For more details see Ref. [Sch11].

For the systems studied in this work, the ground-state calculations are performed using the singe-site MPS algorithm explained above. As a starting point, a random MPS is chosen. The convergence to the ground-state is checked via the variance ${ }^{13}$

$$
\left\langle E_{0}\left|\left(H-E_{0}\right)^{2}\right| E_{0}\right\rangle=\left\langle E_{0}\left|H^{2}\right| E_{0}\right\rangle-\left(\left\langle E_{0}|H| E_{0}\right\rangle\right)^{2},
$$

which is in all cases smaller than $<10^{-6}$ and therefore well controlled. Note that, in this thesis, ground-state calculations are not the bottleneck in the numerics since they mainly provide the input for the more challenging computation of zero-temperature spectral functions.

\section{\begin{tabular}{l|l} 
Variational compression of MPS & 3.3 .4
\end{tabular}}

Some frequently used operations on MPS lead to considerably increased dimensions of the resulting MPS matrices. This issue is of significant importance in MPS-based frequencydomain methods. More general examples are the global application of an MPO to an MPS or the addition of two or even more MPS. Apart from the aforementioned SVD compression, there also exists the possibility to compress an MPS $|\Psi\rangle$ with increased internal bond dimensions $m^{\prime}$ variationally. In this case, one starts from an MPS $|\tilde{\Psi}\rangle$ of the desired as well as smaller dimension $m<m^{\prime}$.

The variational compression can be formulated as a minimization problem for the squared norm difference

$$
\||\Psi\rangle-|\tilde{\Psi}\rangle \|^{2}=\langle\Psi \mid \Psi\rangle-\langle\Psi \mid \tilde{\Psi}\rangle-\langle\tilde{\Psi} \mid \Psi\rangle+\langle\tilde{\Psi} \mid \tilde{\Psi}\rangle
$$

\footnotetext{
${ }^{11}$ The Jacobi-Davidson method represents a frequently used alternative [SdV96].

${ }^{12}$ There have been proposals of how to avoid such problems in single-site DMRG algorithms [Whi05, HMSW15].

${ }^{13}$ Here $\left|E_{0}\right\rangle$ and $E_{0}$ denote the numerically determined results.
} 
with respect to $|\tilde{\Psi}\rangle$, i.e., its matrix entries $\tilde{M}^{s_{l}}$. One possibility is to choose the initial guess $|\tilde{\Psi}\rangle$ as a random MPS. The optimization of a local matrix $\tilde{M}^{s_{l}}$ is again achieved by iteratively sweeping back and forth along the chain. In this sweeping procedure the other matrices are kept constant. After a few sweeps the approximation $|\tilde{\Psi}\rangle$ will usually have converged.

For updating $\tilde{M}^{s_{i}}$ at the $i$-th site, it is sufficient to regard $\langle\tilde{\Psi} \mid \tilde{\Psi}\rangle-\langle\tilde{\Psi} \mid \Psi\rangle$ as the matrix only appears in these contributions. It is evident that this represents a highly nonlinear minimization problem because one extremizes with respect to $\tilde{M}^{s_{l},(l \neq i)}$ on all other sites $(l \neq i)$. This leads to

$$
\frac{\partial}{\partial \tilde{M}_{a_{l-1}, a_{l}}^{s_{l},(l \neq i)}}(\langle\tilde{\Psi} \mid \tilde{\Psi}\rangle-\langle\tilde{\Psi} \mid \Psi\rangle)=0 .
$$

A solution of this problem is derived in Ref. [Sch11]. In the following the implementation of the solution is reviewed. First of all, it is beneficial to represent the ansatz MPS $|\tilde{\Psi}\rangle$ with the desired internal bond dimensions in a mixed-canonical form, see Eq. (3.19). The MPS can be brought into this form by a series of computationally cheaper QR decompositions since the additional information provided by an SVD is not needed at this point. Assuming that the matrices are of the form $\tilde{A}^{s_{1}} \cdots \tilde{A}^{s_{L-1}} \tilde{M}^{s_{L}}$, the unnormalized matrix $\tilde{M}^{s_{L}}$ is updated. ${ }^{14}$ Then a $\mathrm{QR}$ decomposition is used to shift the unnormalized matrices one site to the left, i.e., $\tilde{A}^{s_{1}} \cdots \tilde{A}^{s_{L-2}} \tilde{M}^{s_{L-1}} \tilde{B}^{s_{L}}$. As a next step, the matrix $R_{a_{L}, a_{L}^{\prime}}$ needed to update $\tilde{M}^{s_{L-1}}$ is calculated by iteratively contracting

$$
R_{a_{i}, a_{i}^{\prime}}=\left(\sum_{s_{i}} \tilde{M}^{s_{i}}\left(\cdots\left(\sum_{s_{L}} \tilde{M}^{s_{L}} M^{s_{L} \dagger}\right) \cdots\right) M^{s_{i} \dagger}\right)_{a_{i}, a_{i}^{\prime}} .
$$

At this site only the innermost sum is performed. The general form of Eq. (3.40) shows that it is nothing else than the partial evaluation of an overlap (see Eq. (3.26)). Also note that $R_{a_{i}, a_{i}^{\prime}}$ will be reused and hence inserted in Eq. (3.40) to iteratively compute $R_{a_{i-1}, a_{i-1}^{\prime}}$ at a later point. In the current step, $\tilde{M}^{s_{L-1}}$ can now be updated by

$$
\tilde{M}_{a_{i-1}, a_{i}}^{s_{i}}=\sum_{a_{i-1}^{\prime}} L_{a_{i-1}, a_{i-1}^{\prime}}\left(\sum_{a_{i}^{\prime}} R_{a_{i}, a_{i}^{\prime}} M_{a_{i-1}^{\prime}, a_{i}^{\prime}}^{s_{i}}\right) .
$$

If one starts to optimize the $\tilde{M}^{s_{l}}$ matrices in a right-to-left sweep, as explained here, it is necessary to calculate the $L_{a_{i-1}, a_{i-1}^{\prime}}$ matrices beforehand. This is done in the course of the left-to-right sweep used to impose the canonical form at the beginning. These matrices are also computed by iterative contractions: ${ }^{15}$

$$
L_{a_{i-1}, a_{i-1}^{\prime}}=\left(\sum_{s_{i-1}} \tilde{M}^{s_{i-1} \dagger}\left(\cdots\left(\sum_{s_{1}} \tilde{M}^{s_{1} \dagger} M^{s_{1}}\right) \cdots\right) M^{s_{i-1}}\right)_{a_{i-1}, a_{i-1}^{\prime}}
$$

Having updated $\tilde{M}^{s_{L-1}}$, another QR decomposition is applied to shift the unnormalized matrices one site to the left. Afterwards $R_{a_{L}, a_{L}^{\prime}}$ is used to obtain $R_{a_{L-1}, a_{L-1}^{\prime}}$ via Eq. (3.40).

\footnotetext{
${ }^{14}$ For the update Eq. (3.41) is used, but in this case $R$ only is a matrix represented by a scalar one since there is no tripartition of the system.

${ }^{15}$ In Eqs. (3.40) and (3.42), the canonical form of the MPS matrices of $|\tilde{\Psi}\rangle$ is not pointed out by the notation since it is not required for the calculation of these contractions.
} 
Now $\tilde{M}^{s_{L-2}}$ can be updated. Then the procedure is continued by a couple of sweeps. The distance $\||\Psi\rangle-|\tilde{\Psi}\rangle \|^{2}$ between the updated approximation $|\tilde{\Psi}\rangle$ and $|\Psi\rangle$ is evaluated in order to assess the quality of the variational approximation. The sweeping is stopped once it falls below a specified threshold.

For general sums such as $|\Psi\rangle=\sum_{\alpha=1}^{n}\left|\Phi_{\alpha}\right\rangle$ the expression in Eq. (3.39) has to be extended. It then reads

$$
\begin{aligned}
& \frac{\partial}{\partial \tilde{M}_{a_{i-1}, a_{i}}^{s_{l},(l \neq i}}(\langle\tilde{\Psi} \mid \tilde{\Psi}\rangle-\langle\tilde{\Psi} \mid \Psi\rangle) \\
= & \frac{\partial}{\partial \tilde{M}_{a_{i-1}, a_{i}}^{s_{l},(l \neq i)}}\left(\langle\tilde{\Psi} \mid \tilde{\Psi}\rangle-\left\langle\tilde{\Psi} \mid \Phi_{1}\right\rangle-\left\langle\tilde{\Psi} \mid \Phi_{2}\right\rangle-\cdots-\left\langle\tilde{\Psi} \mid \Phi_{n}\right\rangle\right) .
\end{aligned}
$$

As a consequence, the update of $\tilde{M}_{a_{i-1}, a_{i}}^{s_{i}}$ is modified by introducing an additional sum:

$$
\tilde{M}_{a_{i-1}, a_{i}}^{s_{i}}=\sum_{\alpha=1}^{n}\left[\sum_{a_{i-1}^{\prime}} L_{a_{i-1}, a_{i-1}^{\prime}}^{\alpha}\left(\sum_{a_{i}^{\prime}} R_{a_{i}, a_{i}^{\prime}}^{\alpha} M_{a_{i-1}^{\prime}, a_{i}^{\prime}}^{s_{i}, \alpha}\right)\right] .
$$

Here the matrices $L^{\alpha}$ and $R^{\alpha}$ represent the contractions incorporating the MPS matrices $M^{s_{i}, \alpha}$ of the state $\left|\Phi_{\alpha}\right\rangle$ for $\alpha=1, \ldots, n$. These are still obtained via Eqs. (3.40) and (3.42). It is assumed that the states $\left|\Phi_{\alpha}\right\rangle$ have an initial bond dimension of $m$.

Note that a straightforward calculation of $|\Psi\rangle=\sum_{\alpha=1}^{n}\left|\Phi_{\alpha}\right\rangle$ using the ordinary MPS algebra would lead to a result $|\Psi\rangle$ with maximal internal bond dimensions $(n \mathrm{~m})$, which are usually truncated back to $m$ by SVD compressions with a computational effort of $\left(n^{3} m^{3}\right)$ in a single step. Therefore, it is desirable that such an inflated state does not have to be constructed. The update in Eq. (3.44) only includes contractions of matrix product states with the original and final internal bond dimensions $m$. Here the most costly operation is the SVD or QR factorization needed to maintain the mixed-canonical representation of $|\tilde{\Psi}\rangle$. It includes of the order of $m^{3}$ operations for a single decomposition. The example illustrates why an iterative or variational compression is oftentimes more efficient than an SVD compression [Sch11]. In Ref. [Sch11], it is also noted that this advantage is not that decisive for small compressions. However, in this thesis mostly larger compressions are necessary. The reason is that for the frequency-domain methods used in this thesis (see Sec. 3.5 below), one has to cope with states of the form

$$
|\Psi\rangle=A\left|\Phi_{1}\right\rangle+\left|\Phi_{2}\right\rangle+\left|\Phi_{3}\right\rangle
$$

where $A$ denotes an operator with internal MPO dimension $D$, e.g., the Hamiltonian $(D=5)$ or Liouville operator $(D=8)$ for a spin-1/2 Heisenberg chain. Now there is an additional issue since the state $A\left|\Phi_{1}\right\rangle$ has increased internal bond dimensions that are $D m .{ }^{16}$ For memory efficiency, its MPS matrices are only created on the fly for the site that is currently optimized. As for the variational compression, there is no need to perform a QR decomposition on them, these matrices only occur in the evaluation of contractions. Apart from that, the other contractions associated with $\left|\Phi_{2}\right\rangle$ or $\left|\Phi_{3}\right\rangle$ are computed as discussed above.

\footnotetext{
${ }^{16}$ As explained before, under the application of an MPO to an MPS the internal dimensions of the result are the product of the MPO and MPS dimensions.
} 



\section{\begin{tabular}{l|l} 
Time evolution & 3.4
\end{tabular}}

In order to determine the time evolution of a quantum state governed by the timedependent Schrödinger equation (2.5), there are essentially two approaches. The first is to directly integrate the Schrödinger equation, e.g., by the Runge-Kutta method which can also be implemented with MPS [GR06]. The second is the use of the formal solution in Eq. (2.6). Here the time-evolution operator applied to an initial quantum state at time $t_{0}(\hbar=1)$

$$
\left|\Psi\left(t_{0}+\Delta t\right)\right\rangle=\mathrm{e}^{-i H \Delta t}\left|\Psi\left(t_{0}\right)\right\rangle
$$

is discretized on a small time interval $\Delta t$. This allows for a propagation of the initial state by successively applying the time-evolution operator for small time intervals $\Delta t$ :

$$
|\Psi(t)\rangle=\mathrm{e}^{-i H \Delta t} \mathrm{e}^{-i H \Delta t} \cdots \mathrm{e}^{-i H \Delta t}\left|\Psi\left(t_{0}=0\right)\right\rangle,
$$

where the time $t$ is the sum of several time steps $\Delta t$ after setting $t_{0}=0$. Currently, a widely used approach is the adaptive time-dependent DMRG method [WF04, DKSV04] or the time-evolving block decimation [Vid03, Vid04], where a Suzuki-Trotter decomposition [Tro59, Suz76] is used to approximate the exponentials $\mathrm{e}^{-i H \Delta t}$. Another class of algorithms uses a Krylov-space approximation, e.g., via the projection of the time-evolution operator onto the space spanned by a Lanczos basis [Sch04, MMN05]. These approaches are reviewed in Refs. [Sch05b, GR06].

The algorithm mainly used in this thesis is a recent development based on the timedependent variational principle (TDVP) $\left[\mathrm{HLO}^{+} 14\right]$. Therefore, the main goal of this section is to review this approach and only briefly mention other techniques in Sec. 3.4.4. The TDVP time evolution algorithm has been chosen due to its straightforward implementation and the ability to treat long-range interactions, which could be useful for further studies beyond this thesis. ${ }^{17}$

\section{\begin{tabular}{l|l} 
Spectral functions via real-time evolution & 3.4 .1
\end{tabular}}

Besides frequency-domain methods, time-evolution methods have been used extensively in the calculation of spectral functions - both at zero [WF04, WA08] and finite temperature by purifying mixed-state density operators [BSW09, KBM12, KKHM15]. The computation of time-dependent correlation functions $\left\langle S_{L / 2+n}^{\alpha}(t) S_{L / 2}^{\gamma}\right\rangle$ (with e.g. $\alpha=\gamma=z$ ) in real space is used as an input to subsequent Fourier transforms to momentum and frequency space. For instance, the dynamical spin structure factor is obtained by

$$
S_{\alpha \gamma}(k, \omega)=\lim _{\eta \rightarrow 0} \frac{1}{L} \sum_{n} \mathrm{e}^{i k n} \int \mathrm{d} t \mathrm{e}^{i \omega t-\eta t}\left\langle S_{L / 2+n}^{\alpha}(t) S_{L / 2}^{\gamma}\right\rangle,
$$

if the system is large enough and one may assume translational invariance. One can also exploit time translational invariance $\langle A(t) B\rangle=\langle A(t / 2) B(-t / 2)\rangle$. However, accessible time scales are limited by the growth of entanglement in the course of the time evolution. Thus, the accuracy and resolution of the spectral function is restricted by the

\footnotetext{
${ }^{17}$ Long-range interactions can also be incorporated by other approaches based on MPOs [ZMK $\left.{ }^{+} 15\right]$.
} 
maximal accessible time [BSW09, FF10]. In particular, the properties at small frequency depend on the hardly accessible long-time behavior of response functions. Therefore, one oftentimes inserts a finite parameter $\eta$ introducing Lorentzian broadening in Eq. (3.48). One remedy is the extrapolation of time-dependent correlation functions by linear prediction (see Sec.3.5.4). An efficient measure which helps to reduce the entanglement growth at finite temperature and therefore reduces the computational effort is a backward time-evolution on the auxiliary degrees of freedom [KBM12, KBM13]. This unitary transformation merely changes the basis of the auxiliary space and leaves the physical content untouched. Note that the backward time evolution on the auxiliary space can be motivated by the Liouvillian description. There are also further related optimization schemes [Bar13].

Having briefly covered the calculation of spectral functions in the time-domain by DMRG or MPS methods for real-time evolution, one should stress that in this thesis such methods will be used for imaginary-time evolution to accurately obtain purifications of thermal states (see Sec. 3.6.2). An imaginary-time evolution is obtained by the replacement $t \rightarrow$ $-i \tau$. Thermal states are an important prerequisite for the calculation of finite-temperature spectral functions and also for the calculation of static thermodynamic properties.

\section{Time evolution via the time-dependent variational principle}

Compared to other time-evolution approaches in the DMRG or MPS context, this approach is rather new and only recently a simplified as well as promising algorithm could be formulated in Ref. $\left[\mathrm{HLO}^{+} 14\right]$. Thus, the following presentation is based on this reference. The conceptual idea is the formulation of the Dirac-Frenkel time-dependent variational principle (TDVP) reviewed in Ref. [LEK72] for the variational ansatz class of MPS $\left[\mathrm{HCO}^{+} 11\right.$, LRSV13].

From a geometrical point of view, as illustrated in Fig. 3.3, the TDVP corresponds to an orthogonal projection of the time-evolution vector $-i H|\Psi[M]\rangle$ onto the tangent space of the MPS manifold $\mathcal{M}_{\mathrm{MPS}}$ at the state $|\Psi[M]\rangle$

$$
\frac{\mathrm{d}|\Psi[M]\rangle}{\mathrm{d} t}=-i P_{T_{|\Psi[M]\rangle} \mathcal{M}_{\mathrm{MPS}}} H|\Psi[M]\rangle .
$$

Here the argument in the square brackets denotes the set of MPS matrices. According to Ref. [LOV15], the projection operator onto the tangent space can be expressed as

$$
P_{T_{|\Psi[M]\rangle} \mathcal{M}_{\mathrm{MPS}}}=\sum_{l=1}^{N} P_{A}^{[1: l-1]} \otimes I_{l} \otimes P_{B}^{[l+1: L]}-\sum_{l=1}^{L-1} P_{A}^{[1: l]} \otimes P_{B}^{[l+1: L]},
$$

where the projectors

$$
P_{A}^{[1: l]}=\sum_{\alpha}\left|\Phi_{\alpha}^{[1: l]}\right\rangle_{A A}\left\langle\Phi_{A, \alpha}^{[1: l]}\right| \quad \text { and } \quad P_{B}^{[l: L]}=\sum_{\beta}\left|\Phi_{\beta}^{[l: L]}\right\rangle_{B B}\left\langle\Phi_{B, \beta}^{[l: L]}\right|
$$

on the left (right) block of the lattice are stated with respect to the associated orthonormal basis sets $\left|\Phi_{\alpha}^{[1: l]}\right\rangle_{A}\left(\left|\Phi_{\beta}^{[l+1: L]}\right\rangle_{B}\right)$.

From this, a key feature of TDVP time evolution follows directly: the time-evolved state will always remain in the manifold. Therefore, it is justified to directly time-evolve the 


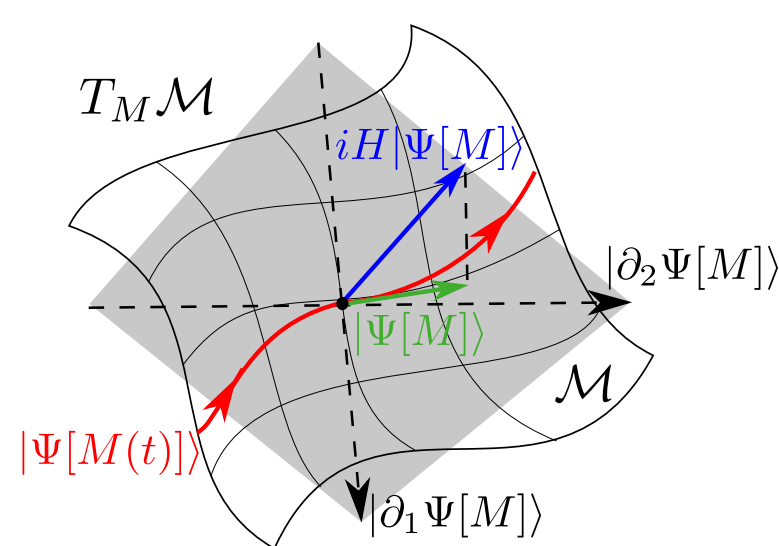

Figure 3.3: (Figure inspired by Fig. 1 in Ref. $\left[\mathrm{HCO}^{+} 11\right]$.) Sketch illustrating the MPS manifold $\mathcal{M}=\mathcal{M}_{\mathrm{MPS}}$ (curved surface) and the tangent space $T_{M} \mathcal{M}=T_{|\Psi[M]\rangle \mathcal{M}_{\mathrm{MPS}}}$ (gray plane) spanned by the coordinate axes $\left|\partial_{1} \Psi[M]\right\rangle$ and $\left|\partial_{2} \Psi[M]\right\rangle$ which are in general not orthogonal. The direction $i H|\Psi[M]\rangle$ of time evolution (blue arrow) is best approximated by the orthogonal tangent-space projection (green head). The optimal trajectory $|\Psi[M(t)]\rangle$ is denoted by the red curve.

parameters of the MPS which is formally represented by $|\Psi[M(t)]\rangle$ including the time dependence for the set of MPS matrices as an argument. This is an important conceptual difference to time-evolution approaches based on a Suzuki-Trotter splitting [Tro59, Suz76] of the Hamiltonian (see Sec. 3.4.4). In these cases, the time-evolved state leaves the MPS manifold upon applying the Trotter time step, but has to be approximated within it subsequently.

The original TDVP-based algorithm $\left[\mathrm{HCO}^{+} 11\right]$ solved the system of non-linear coupled equations arising from Eq. (3.49) by using an explicit integration scheme. This procedure results in a simultaneous update of all MPS parameters, which is different from the DMRG philosophy of local updates and requires the calculation of explicit inverses limiting the applicability of this approach.

The crucial insight leading to a much simpler algorithm, which is very similar to the ground-state DMRG algorithm, is that each term in Eq. (3.50) can be integrated exactly [LOV15]. For instance, one may consider the term $P_{A}^{[1: l-1]} \otimes I_{l} \otimes P_{B}^{[l+1: L]}$ and $|\Psi[M]\rangle$ in the mixed canonical form with a single-site center block $M_{C}(l)$ at site $l$. Such a center block is simply $M_{\left(s_{l}, a_{l-1}\right), a_{l}}$ which is obtained by fusing indices. As mentioned earlier, one now has to deal with the time dependence of the MPS parameters. This is achieved by including the time dependence in the center block $M_{C}(l, t)$, which fulfills

$$
\dot{\boldsymbol{M}}_{C}(l, t)=-i H(l) \boldsymbol{M}_{C}(l, t) .
$$

Following Ref. $\left[\mathrm{HLO}^{+} 14\right]$, we adopt a bold notation to denote whenever the matrix $M_{C}(l, t)$ is represented as a vector. The formal solution of this equation is obtained as

$$
\boldsymbol{M}_{C}(l, t)=\mathrm{e}^{-i H(l) t} \boldsymbol{M}_{C}(l, 0) .
$$

The operator $H(l)$ is the one-site effective Hamiltonian. Analogously, the projectors $-P_{A}^{[1: l]} \otimes P_{B}^{[l+1: L]}$ can be integrated. To this end, $|\Psi\rangle$ is considered in the mixed canonical form, in which all parameters are absorbed into the left and right blocks, except for the degrees of freedom contained in the zero-site center block $C(l)$ on the bond between sites $l$ and $l+1$. The singular values of $C(l)$ are the Schmidt coefficients of this state with respect to the bipartition. Here the formal solution is given by

$$
\boldsymbol{C}(l, t)=\mathrm{e}^{+i K(l) t} \boldsymbol{C}(l, 0) .
$$

The zero-site effective Hamiltonian with respect to the bases $\left|\Phi_{\alpha}^{[1: l]}\right\rangle_{A}$ and $\left|\Phi_{\beta}^{[l+1: L]}\right\rangle_{B}$ is denoted by $K(l)$. The positive sign in the exponential in Eq. (3.54) suggests a backward time evolution. 
In order to treat the full projector consisting of integrable parts in Eq. (3.50), this approach employs a Trotter splitting of the tangent space projector. Each individual projector considered above is then evolved for a small time step $\Delta t$. Similar to DMRG, the algorithm includes a sweeping $\operatorname{protocol}^{18}$ starting with a right-canonical MPS at $l=1$ :

1. Evolve $M_{C}(l)$ via Eq. (3.53) for a time step $\Delta t$.

2. Factorize the result $M_{C}(l)=A_{C}(l) C(l)$ and split $A_{C}(l)$ into the new $A_{l}^{s}$.

3. Backward time evolution of $C(l)$ for a step $\Delta t$ using Eq. (3.54).

4. Multiply the result $C(l)$ to the right by $C(l) B^{s_{l+1}}$ and obtain $M_{C}(l+1)$ by merging.

5. Continue with the first step.

A following right-to-left sweep leads to a second-order scheme yielding the time-evolved state $|\Psi[M(t+\Delta t)]\rangle$ with an error of the order $\mathcal{O}\left(\Delta t^{3}\right)$. This error is associated with the splitting scheme of the tangent space projector. As a matter of convention, the time step $\Delta t$ is specified for one full sweep, i.e, the state is propagated by $\Delta t / 2$ in, e.g., one left-to-right sweep. The errors occurring in the numerical application of the exponentials in Eqs. (3.53) and (3.54) by the Lanczos algorithm ${ }^{19}$ (see Sec. 3.2 below) are significantly smaller and can therefore be neglected. Another source of possible inaccuracies is the error with respect to the time evolution described by the original Schrödinger equation. This is assessed by performing the calculations for several values of the MPS bond dimension. For time-independent Hamiltonians, the algorithm from Ref. $\left[\mathrm{HLO}^{+} 14\right]$ outlined above conserves the energy and the norm of the evolved state.

In this thesis, the approach will be used for imaginary-time evolution, i.e., $t \rightarrow-i \tau$, which allows the use of purely real arithmetics. Generally, imaginary-time evolution can also be used as a projection method to find ground states. While this method is rather inefficient compared to the single-site DMRG algorithm discussed in Sec. 3.3.3, it is important for the connection between the simplified TDVP algorithm and DMRG. It turns out that the TDVP algorithm for imaginary-time evolution is formally identical to single-site DMRG in the limit $\Delta \tau \rightarrow \infty\left[\mathrm{HLO}^{+} 14\right]$. This insight into the concealed connection to DMRG-like algorithms also marked important progress in the conceptual understanding of MPSbased time-evolution methods in the past two years. The TDVP formulation for the time evolution of MPS is conceptually of similar importance than the realization that DMRG is basically an application of the time-independent variational principle to MPS.

\section{\begin{tabular}{l|l} 
Krylov-space time evolution & 3.4 .3
\end{tabular}}

Another class of DMRG time-evolution methods is based on the projection of the timeevolution operator $\mathrm{e}^{-i H \Delta t}$ for a small time step $t \rightarrow t+\Delta t$ onto a Krylov subspace [Sch04, MMN05]. These are so-called Krylov-space approaches. Usually the Lanczos algorithm (see Sec. 3.2) is used to generate an orthonormal basis spanning the Krylov subspace to approximate $\mathrm{e}^{-i H \Delta t}$, but generally also the Arnoldi method is an alternative

\footnotetext{
${ }^{18}$ Details for the implementation of this method are given in the supplemental material of Ref. $\left[\mathrm{HLO}^{+} 14\right]$.

${ }^{19}$ In contrast to the Krylov-space projection of exponentials described in Sec. 3.4.3, the Lanczos vectors are represented as real-valued vectors here instead of MPS.
} 
[GR06]. The Lanczos method for time evolution is described in detail in Ref. [NM05]. Formally, the projection of the exponential $\mathrm{e}^{-i H \Delta t}$ onto the Krylov subspace can be written as

$$
\begin{aligned}
|\Psi(t+\Delta t)\rangle & =\mathrm{e}^{-i H \Delta t}|\Psi(t)\rangle \\
& \approx V_{n}(t) \mathrm{e}^{-i T_{n}(t) \Delta t} V_{n}^{\dagger}(t)|\Psi(t)\rangle \\
& =V_{n}(t) U_{n}(t) \mathrm{e}^{-i D_{n}(t) \Delta t} U_{n}^{\dagger}(t) V_{n}^{\dagger}(t)|\Psi(t)\rangle .
\end{aligned}
$$

In the following, the method and the above equations are explained. It proceeds like this:

1. The initial wave function $|\Psi(t)\rangle$ is chosen as the starting vector $\left|f_{0}\right\rangle$ in the recursion relation in Eq. (3.2).

2. After $n$ Lanczos iterations, the tridiagonal matrix $T_{n}(t)$ approximating the Hamiltonian is used to project $\mathrm{e}^{-i H \Delta t}$ onto the Krylov subspace in Eq. (3.56). Here the columns of the matrix $V_{n}(t)$ contain the Lanczos vectors.

3. In the next step, the tridiagonal matrix $T_{n}(t)$ is diagonalized yielding $U_{n}(t) D_{n}(t) U_{n}^{\dagger}(t)$, where $D_{n}(t)$ is a diagonal matrix.

4. Then the time-evolved state $|\Psi(t+\Delta t)\rangle$ and the related observables are calculated.

Note that in this thesis an MPS formulation of this algorithm is used [GR06]. Due to the ability to add matrix product states (see Sec. 3.3.2), Equation (3.57) is implemented as the sum

$$
|\Psi(t+\Delta t)\rangle=\sum_{k=0}^{n}\left[\mathrm{e}^{-i \Delta t T_{n}(t)}\right]_{k, 0}\left|f_{k}\right\rangle .
$$

Here the Lanczos vectors $\left|f_{k}\right\rangle$ are represented as MPS. Thus, their internal dimensions add under addition and it is necessary to compress the resulting MPS to an internal bond dimension of $m$. Here this is implemented as an SVD compression which represents the main source of errors. On the other hand, only a rather small number of Lanczos iterations is needed to ensure a high accuracy of the Krylov space projection, i.e., $n \leq 20$ iterations are usually sufficient [HL97, HL99, MVL03]. In this thesis, a rather small time step of $\Delta t=0.005$ is used which only requires at most $n=7$ to enforce $\left[\mathrm{e}^{-i \Delta t T_{n}(t)}\right]_{n, 0} \lesssim 10^{-12}$ for the matrix entry in Eq. (3.58).

The Lanczos time-evolution method just described was employed for the imaginary-time evolution in the first publication related to this thesis [TMPH14] since the TDVP algorithm explained in the previous section had not yet been introduced at that time. The errors of both methods are shown for the specific heat of a $X X$ chain in Fig. 5.1(b) in Sec. 5.2.

\section{\begin{tabular}{l|l} 
Other time-evolution methods & 3.4 .4
\end{tabular}}

A very popular approach is pursued in the adaptive time-dependent DMRG method [WF04, DKSV04] or the time-evolving block decimation [Vid03, Vid04], where a SuzukiTrotter decomposition [Tro59, Suz76] is used to split a Hamiltonian with nearest-neighbor interactions into terms acting on odd and even bonds

$$
H=H_{\text {odd }}+H_{\text {even }} \text {. }
$$


This can be used to factorize the time-evolution operator, e.g., by a second-order SuzukiTrotter decomposition ${ }^{20}$

$$
\mathrm{e}^{-i H \Delta t} \approx \mathrm{e}^{-i H_{\text {odd }} \Delta t / 2} \mathrm{e}^{-i H_{\text {even }} \Delta t} \mathrm{e}^{-i H_{\text {odd }} \Delta t / 2}+\mathcal{O}\left((\Delta t)^{3}\right)
$$

for a small time step $\Delta t$. Although all terms in $H_{\text {odd }}$ and in $H_{\text {even }}$ commute, one generally has $\left[H_{\text {odd }}, H_{\text {even }}\right] \neq 0$, which introduces errors due to neglected terms. Therefore, the main drawback of approaches based on a Suzuki-Trotter decomposition is that they cannot equally well cope with Hamiltonians including long-range interactions. Implemented in the framework of MPS, the exponentials are represented as MPOs. Being applied, these operators lead to an increase of bond dimensions in the time-evolved MPS. So the MPS matrices need to be truncated by, e.g., an SVD compression after each time step. A more detailed description of this standard method for time evolution is given in Ref. [Sch11]. Moreover, there is the possibility of expanding the time-evolution operator in Chebyshev polynomials using MPS [HWM $\left.{ }^{+} 11\right]$. Although this approach is also interesting due to its ability to treat systems with long-range interactions, it was recently found to be inferior to Krylov- or Trotter-based methods since the accessible times after a global quantum quench in the Bose-Hubbard model have been rather limited [HKM15].

\footnotetext{
${ }^{20}$ In fact, a fourth-order decomposition is chosen in most applications [Sch11, KBM13].
} 


\section{MPS frequency-domain methods at zero temperature}

\section{Introduction $\mid 3.5 .1$}

As mentioned before, DMRG has been extended to the computation of zero-temperature spectral functions in the frequency domain soon after its invention. The first approach of this kind was a continued fraction expansion $(\mathrm{CFE})$ of the dynamical correlation function relying on a DMRG implementation of the Lanczos algorithm [Hal95]. Later another frequency-domain method, namely the correction-vector method [RPK $\left.{ }^{+} 96, \mathrm{KW} 99\right]$, was introduced. Its more efficient formulation in terms of a minimization problem for a functional by Eric Jeckelmann [Jec02] is known as the dynamical density-matrix renormalization-group method (DDMRG). The DDMRG represents an important development in the numerical study of both momentum and frequency-resolved dynamical properties since it provides highly accurate results at $T=0$. It has thereby fostered significant progress. For instance, it has been used to investigate the optical properties of the 1D Hubbard model [JGE00, Jec02, Jec03, SJ09, TVD $\left.{ }^{+} 16\right]$ and to study inelastic neutron scattering spectra of the low-dimensional frustrated quantum magnet azurite [ $\left.\mathrm{JOK}^{+} 11\right]$. However, DDMRG or MPS-based correction-vector [WVS ${ }^{+} 09$ ] calculations are computationally very costly as a separate calculation for each frequency is needed in order to obtain the entire spectral function. Of course, this drawback also allows for easy parallelization, but one would prefer numerical approaches requiring less total resources while giving results for the entire frequency range at one shot. This is certainly one important reason why moment expansions of the dynamical correlation function have been further improved. For instance, the CFE could be improved by an adaptive DMRG scheme for the Lanczos recursion $\left[\mathrm{DHP}^{+} 11\right]$. Independently, the expansion of spectral functions in Chebyshev polynomials, which is known as one variant of the kernel polynomial method (KPM) [WWAF06], has been successfully introduced in the MPS context and also gives very accurate results $\left[\mathrm{HWM}^{+} 11\right]$. In fact, it was not a coincidence that frequency-domain methods could be further improved upon the realization of the intimate links between DMRG and MPS. The reason is that the MPS formulation is better suited for the calculation of excited states $\left[\mathrm{DWH}^{+} 12\right]$. For instance, the excited states generated to determine the expansion coefficients, the Lanczos or Chebyshev vectors, can each be represented by an MPS and the application of, e.g., the Hamiltonian in the recursion is in principle exact. $^{21}$ Therefore, also the adaptive Lanczos method $\left[\mathrm{DHP}^{+} 11\right]$ has been further improved by a reformulation in terms of MPS $\left[\mathrm{DWH}^{+} 12\right]$. Furthermore, it could be stabilized by a subsequent reorthogonalization procedure. This allowed for a very accurate calculation of the dynamical spin structure factor of the spin- $1 / 2$ Heisenberg chain at $T=0\left[\mathrm{DWH}^{+} 12\right]$. Due to the encouraging progress made in the development of frequency-domain methods for the calculation of zero-temperature spectral functions, one main goal of this thesis is to devise MPS frequency-domain methods working at $T>0$. This can be achieved by an extension of existing $T=0$ techniques which are reviewed in the present chapter. It is reasonable to employ MPS approaches based on the expansion of spectral functions giving results over the entire frequency range as they are computationally less demanding than correction-vector approaches. Therefore, the following subsections review the CFE as well as Chebyshev expansions. As the latter technique is mainly used in this work,

\footnotetext{
${ }^{21}$ However, the resulting MPS with increased internal bond dimensions has to be truncated afterwards.
} 
it is discussed in more detail. In particular, there have been quite a few recent developments proposed to increase the spectral resolution of MPS-based Chebyshev expansions $\left[\mathrm{GTV}^{+} 14, \mathrm{WJMS} 15\right]$.

\section{Continued fraction expansion of spectral functions at $T=0 \quad 3.5 .2$}

The calculation of spectral functions by the Lanczos method (see Sec. 3.2) has a long history [HHK72, Hay80, GB87, Dag94]. The dynamical quantities can be determined by starting the recursion in Eq. (3.2) with the vector $\left|f_{0}\right\rangle=A\left|E_{0}\right\rangle / \sqrt{\left\langle E_{0}\left|A^{\dagger} A\right| E_{0}\right\rangle}$. This ensures that only states contributing to the spectral function with non-vanishing spectral weights are generated. The resulting Lanczos coefficients $a_{i}$ and $b_{i}$ then enter a continued fraction expansion (CFE) of a zero-temperature spectral function (see Sec. 2.2.3)

$$
R_{A^{\dagger} A}^{T=0}(z)=-\frac{1}{\pi} \operatorname{Im}\left\langle E_{0}\left|A^{\dagger} \frac{1}{z-\left(H-E_{0}\right)} A\right| E_{0}\right\rangle \approx-\frac{1}{\pi} \operatorname{Im} \frac{\left\langle E_{0}\left|A^{\dagger} A\right| E_{0}\right\rangle}{z-a_{0}-\frac{b_{1}^{2}}{z-a_{1}-\frac{b_{2}^{2}}{z-a_{2}-\ldots}}}
$$

subjected to a finite broadening. Above only positive frequencies are considered, but the treatment of $\omega<0$ is analogous. The derivation of the CFE is contained in Refs. [Ful95, Dag94]. Here $z=\omega+i \eta$ denotes a complex frequency yielding a Lorentzian broadening of the spectral function for finite $\eta$. If one is interested in a smooth spectral function, the broadening has to be chosen large enough such that the individual delta peaks occurring as a consequence of the finite system size are not visible any more.

The key operation in the Lanczos recursion (3.2) is to apply the Hamiltonian $H\left|f_{i}\right\rangle$. In most cases, this amounts to a matrix-vector multiplication which can be implemented efficiently for sparse matrices. For example, one may exploit the symmetries of the Hamiltonian $^{22}$ and constructs the matrix elements of the Hamiltonian on the fly [Saa03, NM05]. Like this it is possible to treat systems consisting of up to about $N=40$ spin- $1 / 2$ degrees of freedom. The technical explanation of the limitation is that the representation of the Lanczos vectors which are needed at each iteration cannot be kept in computer memory any more.

One approach to resolve the issue is to approximate the Lanczos vectors for 1D models by MPS (see Sec. 3.3.2). In this case, the operation $H\left|f_{i}\right\rangle$ corresponds to the application of an MPO to an MPS. This illustrates the flexibility that one may exploit in the representation of the wave function as long as these operations are performed in an efficient framework. For example, an MPS Lanczos method was used to calculate the zero-temperature dynamical spin structure factor of the spin-1/2 antiferromagnetic Heisenberg chain with high accuracy $\left[\mathrm{DWH}^{+} 12\right]$.

\section{Expansions in Chebyshev polynomials $\mid 3.5 .3$}

Another frequency-domain technique which can be used to compute spectral functions is an expansion in terms of Chebyshev polynomials. In some respects this method is similar to a CFE. For instance, the expansion is also generated by a recursive scheme. Prior to discussing the more complicated cases of MPS-based or operator-valued expansions of

\footnotetext{
${ }^{22}$ This will not be possible for the Hamiltonian in Eq. (4.13) which is mostly studied in this work.
} 
spectral functions in Chebyshev polynomials in Sec. 3.5.4, it is useful to review a few basic properties of expansions in orthogonal polynomials.

\section{Basic properties}

This review of some basic properties follows Ref. [WWAF06] and particularly focuses on the case of Chebyshev expansions. In general terms, one may define a scalar product

$$
\langle g \mid h\rangle_{w}=\int_{a}^{b} w(x) g(x) h(x) \mathrm{d} x
$$

between the integrable functions $g, h:[a, b] \rightarrow \mathbb{R}$, where $w(x)$ denotes a positive weight function. For any such scalar product, there exists a complete set of orthogonal polynomials $p_{n}(x)$ fulfilling the orthogonality relations

$$
\left\langle p_{n} \mid p_{m}\right\rangle_{w}=\delta_{n, m}\left\langle p_{n} \mid p_{n}\right\rangle_{w}
$$

This largely facilitates the expansion of a given function $f(x)$, which is for simplicity assumed to be sufficiently well-behaved, ${ }^{23}$ in terms of the polynomials $p_{n}(x)$. The corresponding expansion is given by

$$
f(x)=\sum_{n=0}^{\infty} \alpha_{n} p_{n}(x), \quad \text { where } \alpha_{n}=\left\langle p_{n} \mid f\right\rangle_{w} /\left\langle p_{n} \mid p_{n}\right\rangle_{w} .
$$

In principle, any complete set of orthogonal polynomials can be used to expand the function $f(x)$. However, in most applications dealing with polynomial expansions the Chebyshev polynomials of the first or second kind represent the preferred choice [Boy89, Riv90]. This is the case due to their good convergence properties and the fact that they are sufficiently easy to compute. Thus, Chebyshev polynomials $T_{n}(x)$ of the first kind are used in this thesis. For brevity, we henceforth refer to them as Chebyshev polynomials. The $T_{n}(x)$ are obtained for the weight function $v(x)=\left(\pi \sqrt{1-x^{2}}\right)^{-1}$ in Eq. (3.62) and are defined on the interval $I=[-1,1]$. They thus fulfill the orthogonality relations

$$
\left\langle T_{n} \mid T_{m}\right\rangle_{v}=\frac{1+\delta_{n, 0}}{2} \delta_{n, m}
$$

Using trigonometric functions, the Chebyshev polynomials can be represented explicitly

$$
\begin{aligned}
T_{n}(x) & =\cos [n \arccos (x)] \\
& =\cosh [n \operatorname{arccosh}(x)],
\end{aligned}
$$

which explains their, in principle, easy computation. For many numerical applications as in this thesis, the generation of the Chebyshev polynomials by means of the recursion relation

$$
T_{n+1}(x)=2 x T_{n}(x)-T_{n-1}(x), \quad T_{1}(x)=x, \quad T_{0}(x)=1
$$

is used. ${ }^{24}$ Now we briefly review some basic properties of the Chebyshev polynomials. From the above Eq. (3.67) it is evident that the polynomials $T_{n}(x)$ are even functions for

\footnotetext{
${ }^{23}$ For instance, one may think of a piecewise smooth and continuous function.

${ }^{24}$ MPS-based Chebyshev expansions of spectral functions, which are operator-valued, also make use of the recursion relation in Eq. (3.67). However, they will turn out to be computationally more demanding (see Sec. 3.5.4).
} 

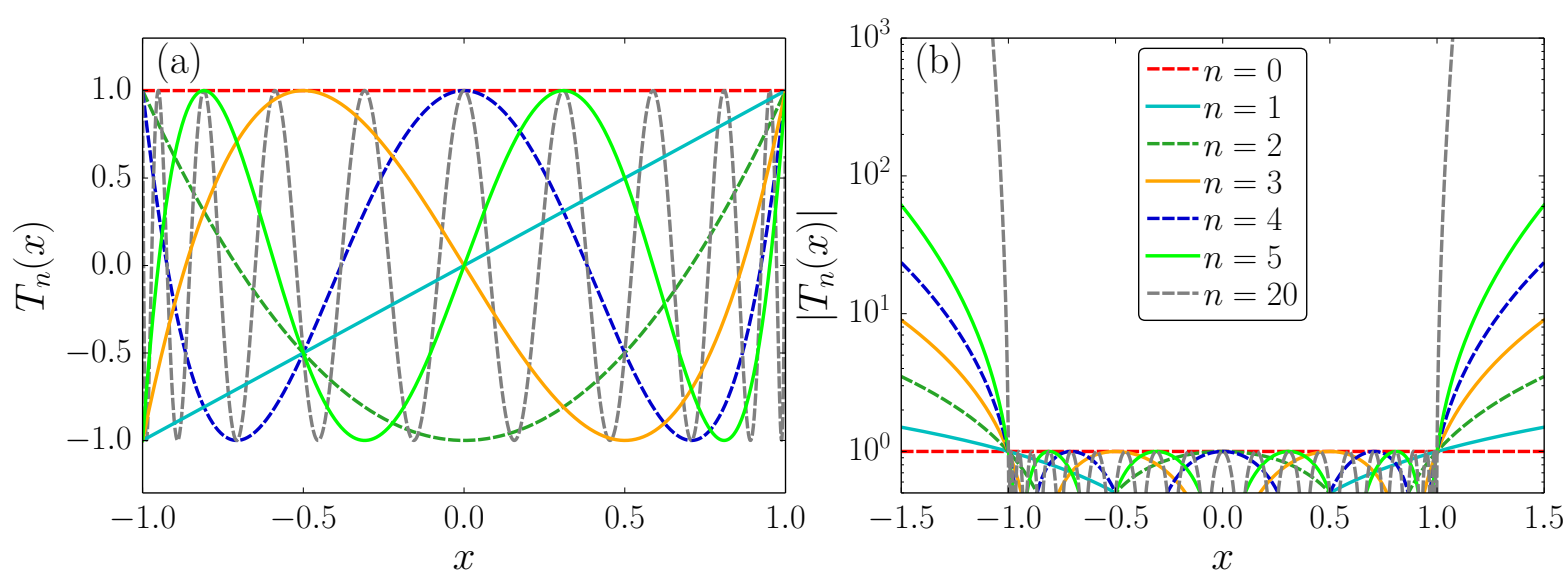

Figure 3.4: Chebyshev polynomials $T_{n}(x)$ of the first kind for selected values of $n$. (a) $T_{n}(x)$ depicted on the interval $I=[-1,1]$ to illustrate the oscillating nature for higher values of $n$ and the boundedness $\left|T_{n}(x)\right| \leq 1$ on $I$. (b) The absolute value $\left|T_{n}(x)\right|$ of the Chebyshev polynomials increases very rapidly for $n>0$ and $x \notin I$.

even numbers of $n$, whereas they are odd functions for odd $n$. Moreover, their trigonometric nature in Eq. (3.66a) reveals that Chebyshev polynomials of higher order $n$ are heavily oscillating functions as depicted in Fig. 3.4(a). Another feature is their boundedness $\left|T_{n}(x)\right| \leq 1$ for $x \in I$ on the interval $I=[-1,1]$. As illustrated in Fig. 3.4(b), the absolute value $\left|T_{n}(x)\right|$ increases very rapidly for $n>0$ and $x \notin I$. In order to prevent a Chebyshev expansion from diverging, one therefore works on a slightly smaller interval, $[-1+\epsilon, 1-\epsilon]$ with $\epsilon>0$, for the implementation of numerical methods.

From Eq. (3.64) it becomes clear that expanding a function $f(x)$ with $x \in I$ in Chebyshev polynomials of the first kind leads to an expansion of the form

$$
f(x)=\sum_{n=0}^{\infty} \frac{\left\langle T_{n} \mid f\right\rangle_{v}}{\left\langle T_{n} \mid T_{n}\right\rangle_{v}} T_{n}(x)=\alpha_{0}^{\prime}+2 \sum_{n=1}^{\infty} \alpha_{n}^{\prime} T_{n}(x),
$$

where the coefficients are given by

$$
\alpha_{n}^{\prime}=\left\langle T_{n} \mid f\right\rangle_{v}=\int_{-1}^{1} \frac{T_{n}(x) f(x)}{\pi \sqrt{1-x^{2}}} \mathrm{~d} x .
$$

\section{Modified moments}

In many numerical applications as, for instance, in MPS-based Chebyshev expansions one would like to avoid integrations over weight functions since they complicate the calculation of expansion coefficients. Thus, one rearranges the expansion by choosing the slightly modified orthogonal functions

$$
\phi_{n}(x)=\frac{T_{n}(x)}{\pi \sqrt{1-x^{2}}}
$$

instead the $T_{n}(x)$. In addition, resorting to the weight function $u(x)=\pi \sqrt{1-x^{2}}$ in the scalar product, ${ }^{25}$ ensures that the orthogonality relations of the functions $\phi_{n}(x)$

$$
\left\langle\phi_{n} \mid \phi_{m}\right\rangle_{u}=\frac{1+\delta_{n, 0}}{2} \delta_{n, m}
$$

\footnotetext{
${ }^{25}$ The weight function $u(x)$ corresponds to the Chebyshev polynomials of the second kind.
} 
are the same as in Eq. (3.65). This leads to a rearranged expansion of the form

$$
f(x)=\sum_{n=0}^{\infty} \frac{\left\langle\phi_{n} \mid f\right\rangle_{u}}{\left\langle\phi_{n} \mid \phi_{n}\right\rangle_{u}} \phi_{n}(x)
$$

which may also be expressed in terms of the Chebyshev polynomials $T_{n}(x)$ :

$$
f(x)=\frac{1}{\pi \sqrt{1-x^{2}}}\left[\mu_{0}+2 \sum_{n=1}^{\infty} \mu_{n} T_{n}(x)\right], \quad \text { with } \mu_{n}=\int_{-1}^{1} T_{n}(x) f(x) \mathrm{d} x .
$$

The result in Eq. (3.73) above represents an important interim result of this section. This is the case since the modified expansion coefficients $\mu_{n}$ can now be evaluated efficiently using the recursive scheme in Eq. (3.67). They are commonly known as Chebyshev moments.

Numerical approaches as pursued in this thesis oftentimes only allow for the calculation of a finite number $N$ of coefficients. Given this constraint, one wants to find the best approximation to a function $f(x)$ by a polynomial series expansion of some finite maximal order. A simple truncation of the infinite series (see Eq. (3.73))

$$
f(x) \approx f_{N}(x)=\frac{1}{\pi \sqrt{1-x^{2}}}\left[\mu_{0}+2 \sum_{n=1}^{N-1} \mu_{n} T_{n}(x)\right]
$$

leads to inaccuracies such as oscillations. These occur close to points where $f(x)$ is, e.g., not continuously differentiable. The approximation of functions $f(x)$ displaying discontinuities or singularities is still harder since the oscillations, also referred to as Gibbs oscillations, become worse in such circumstances. In order to illustrate these undesirable effects near singularities or jumps, we consider the truncated Chebyshev expansions $f_{N}(x)$ of the Dirac delta function $\delta(x)$ and a step function which are each represented by the solid line in Figs. 3.5(a)-(b) further below. In both cases the expansions are truncated after $N=100$ moments.

\section{The kernel polynomial method}

One established way to remove the artificial Gibbs oscillations is the convolution of the truncated series in Eq. (3.74) with a kernel $K_{N}(x, y)$ :

$$
f_{N}^{\mathrm{KPM}}(x)=\int_{-1}^{1} \pi \sqrt{1-y^{2}} K_{N}(x, y) f_{N}(y) \mathrm{d} y=\left\langle K_{N}(x, y) \mid f_{N}(y)\right\rangle_{u},
$$

where $x, y \in[-1,1]$. This procedure is known as the kernel polynomial method (KPM) [WWAF06]. Note that $K_{N}(x, y)$ depends on the number of retained moments $N$. One preferably uses kernels granting uniform convergence between $f(x)$ and $f_{N}^{\mathrm{KPM}}(x)$ with respect to the supremum norm $\|\cdot\|_{\infty}$ on an interval, i.e., $-1+\epsilon<x<1-\epsilon$. Mathematically, this reads

$$
\left\|f-f_{N}^{\mathrm{KPM}}\right\|_{\infty}=\sup _{-1+\epsilon<x<1-\epsilon}\left|f(x)-f_{N}^{\mathrm{KPM}}(x)\right| \rightarrow 0 \quad \text { for } N \rightarrow \infty .
$$

For a detailed review on several proposed kernels and the conditions they have to satisfy for uniform convergence refer to Ref. [WWAF06]. In practice, one of these required 
conditions is the positivity of the kernel $K_{N}(x, y)>0 \forall x, y \in[-1,1]$. It enforces that approximations of positive functions also assume positive values. An important property of the KPM is that the integral of the original function $f(x)$ equals that of its approximation $f_{N}^{\mathrm{KPM}}$, i.e.,

$$
\int_{-1}^{1} f(x) \mathrm{d} x=\int_{-1}^{1} f_{N}^{\mathrm{KPM}}(x) \mathrm{d} x
$$

This is guaranteed by the normalization condition $\int_{-1}^{1} K_{N}(x, y) \mathrm{d} x=\phi_{0}(y)$, being equivalent to $g_{0}=1$. Equation (3.77) can be used whenever a physical sum rule is checked after a computation.

The convolution in Eq. (3.75) introduces real-valued damping factors $g_{n}$, which amounts to another important modification of the series expansion:

$$
f_{N}^{\mathrm{KPM}}(x)=\sum_{n=0}^{N-1} g_{n} \frac{\left\langle\phi_{n} \mid f\right\rangle_{u}}{\left\langle\phi_{n} \mid \phi_{n}\right\rangle_{u}} \phi_{n}(x)=\frac{1}{\pi \sqrt{1-x^{2}}}\left[g_{0} \mu_{0}+2 \sum_{n=1}^{N-1} g_{n} \mu_{n} T_{n}(x)\right] .
$$

In this thesis, we use the Jackson kernel yielding the damping coefficients

$$
g_{n}^{J}=\frac{(N-n+1) \cos \frac{\pi n}{N+1}+\sin \frac{\pi n}{N+1} \cot \frac{\pi}{N+1}}{N+1},
$$

which are depicted for $N=100$ in Fig. 3.5(c). In order to assess the effect of Jackson damping, one may regard the convolution of a Dirac delta function $\delta(x-\bar{x})$ shifted to $\bar{x} \in[-1,1]$ by the Jackson kernel. For $N=100$ and $\bar{x}=0$, the resulting Chebyshev expansion is depicted as the dashed line in Fig. 3.5(a). The oscillations have evidently been suppressed at the expense of an introduced broadening which reduces the spectral resolution. In order to better understand this, it can be shown [WWAF06] that the delta peak is subjected to nearly Gaussian broadening and its approximation is therefore given by

$$
\delta_{N}^{\mathrm{KPM}}(x-\bar{x}) \approx \frac{1}{\sqrt{2 \pi \eta^{2}}} \exp \left(-\frac{(x-\bar{x})^{2}}{2 \eta^{2}}\right) .
$$

Most importantly, note that the Gaussian broadening $\eta$ in Eq. (3.80) depends on both $N$ and also the position of the delta function and is thus not uniform throughout the interval:

$$
\eta(N, \bar{x}) \approx \frac{\pi}{N} \sqrt{1-\bar{x}^{2}}
$$

Jackson damping is usually a very good choice as a continuous function can be approximated up to errors of $\mathcal{O}(1 / N)$ [Jac11, Jac12, WWAF06]. Moreover, this damping scheme is optimized for high resolution. Thus, most of the intensity belonging to peaks in the spectral function is found very close to the peak position since a Gaussian function has rapidly decaying tails.

The Lorentzian kernel presents an important alternative since Lorentzian broadening is intrinsic to some approaches for the calculation of dynamical correlation functions. Thus, the Chebyshev expansions of the Dirac delta function and a step function obtained for Lorentz damping and $N=100$ are also shown in Figs. 3.5(a)-(b). The damping factors for this case are

$$
g_{n, \lambda}^{L}=\frac{\sinh \left[\lambda\left(1-\frac{n}{N}\right)\right]}{\sinh \lambda}
$$



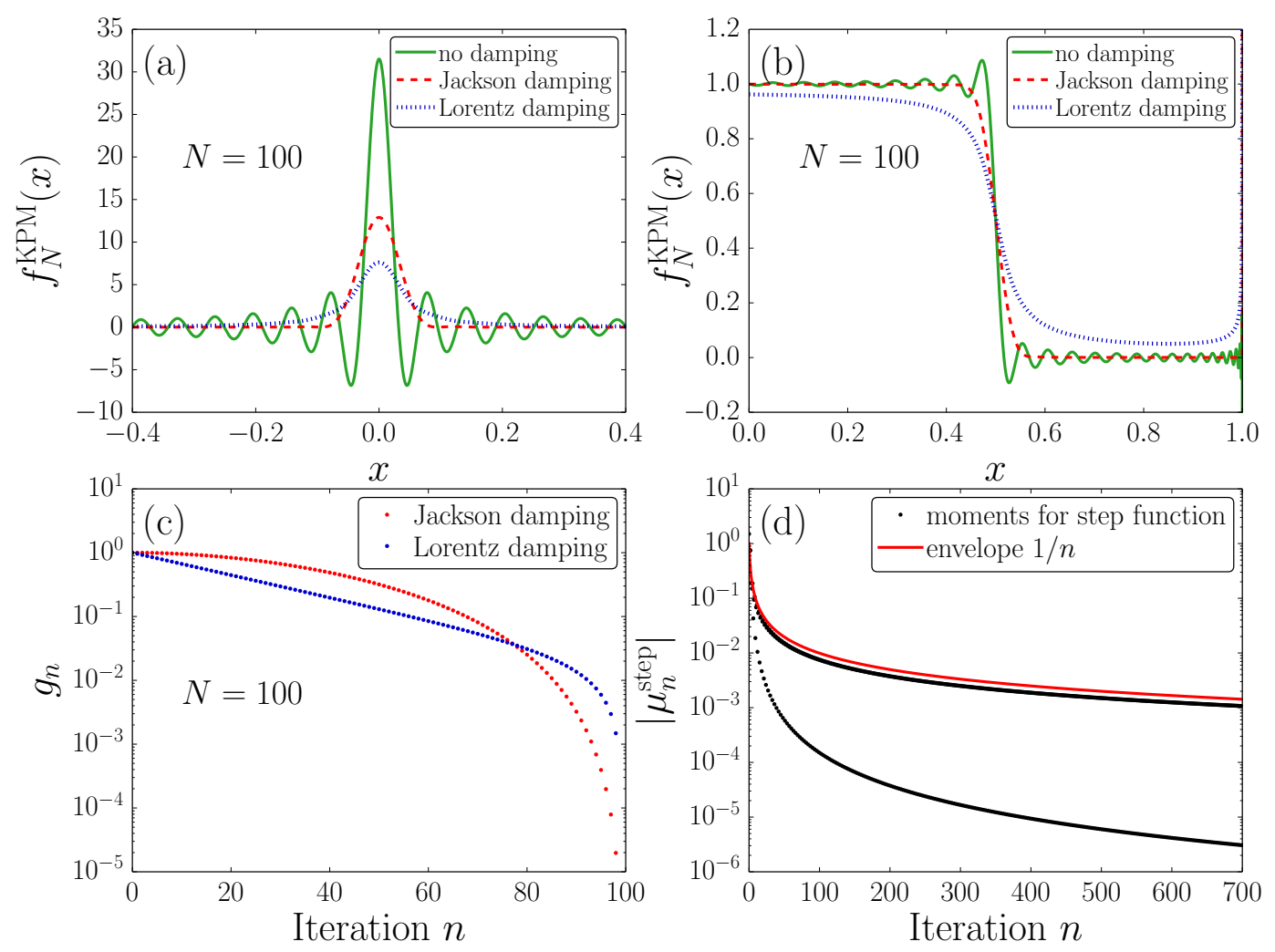

Figure 3.5: (a) Chebyshev expansions (using Eq. (3.78)) of order $N=100$ of (a) the Dirac delta function $\delta(x)$ and (b) a step function illustrating three different damping schemes in the undesirable presence of singularities or jumps. In the case of no damping $\left(g_{n} \equiv 1\right)$ unwanted Gibbs oscillations are present. They can be significantly cured by the convolution of the truncated expansion in Eq. (3.74) with a broadening kernel. Here results employing the Jackson respectively the Lorentz kernel are shown. (c) Associated damping factors $g_{n}$ for $N=100$ as a function of the iteration number $n$. (d) Absolute value $\left|\mu_{n}^{\text {step }}\right|$ of the first 700 Chebyshev moments obtained in the expansion of the step function. In this case, the envelope of the moments converges algebraically with $1 / n$ for $n \gg 1$ (see Sec. 3.5.3). Note the oscillatory behavior of the moments.

which are depicted for $N=100$ in Fig. 3.5(c). Here $\lambda=4$ is adopted following Refs. [WWAF06, HWM $\left.{ }^{+} 11\right]$. This choice for $\lambda$ will cause a delta function $\delta(x-\bar{x})$ to be broadened into a nearly Lorentzian function

$$
\delta_{N}^{\mathrm{KPM}, \mathrm{L}}(x-\bar{x}) \approx \frac{1}{\pi} \frac{\eta_{L}}{(x-\bar{x})^{2}+\eta_{L}^{2}}
$$

of width $\eta_{L}(N, \bar{x})=\sqrt{1-\bar{x}^{2}} \lambda / N$.

\section{Convergence of Chebyshev moments}

In order to discuss the rate of convergence of the Chebyshev moments for a function $f(x)$ in the limit $n \rightarrow \infty$, it is very helpful to note the very close connection between Chebyshev expansions and Fourier series [Boy89]. In fact, it can be shown by a change of variable, $x=\cos \theta$, that the polynomials $T_{n}(x)$ in a Chebyshev expansion are equivalent to the basis functions used in a Fourier cosine series

$$
T_{n}(\cos \theta)=\cos (n \theta) .
$$


Thus, Chebyshev polynomial expansions are equivalent to Fourier cosine series under this change of variable. The convergence properties of Chebyshev expansions are therefore very similar to those of Fourier series. For a review of this issue refer to Ref. [Boy89]. The convergence of the Chebyshev moments $\mu_{n}$ of a function $f(x)$ depends on the highest integer $k$ for which its $k$-th derivative $f^{(k)}(x)$ is integrable. For finite $k$, there is algebraic convergence and the envelope exhibits a decay by at least $1 / n^{k}{ }^{26}$ For a smooth function $f(x), k=\infty$, the envelope of the moments $\mu_{n}$ even exhibits exponential convergence with respect to $n$. Thus, in the case of a step function $(k=1)$, the envelope has an order of convergence of about $1 / n$ and is also depicted in Fig. 3.5(d) to verify this result. For a delta function $(k=0)$, the Chebyshev moments oscillate without any decay. This or slow algebraic decay in the presence of discontinuities leads to unfavorable Gibbs oscillations if the Chebyshev expansion is truncated (see Eq. (3.74)). Most importantly, the asymptotic behavior discussed in this paragraph only holds for $n \gg 1$ and not for small or moderate $n$ [Boy89]. With this knowledge about the convergence of the $\mu_{n}$ for intermediate $n$, it is easier to understand the recent developments to improve the resolution of Chebyshevexpanded spectral functions in the next section.

\section{Chebyshev expansion of spectral functions at $T=0 \quad 3.5 .4$}

In the following it is explained how the kernel polynomial method (KPM) is used to expand zero-temperature spectral functions in an MPS framework. This results in an operatorvalued Chebyshev expansion and some other complications arising, e.g., from the fact that a Chebyshev expansion only grants convergence in the interval $[-1,1]$. The presentation of such issues in the current section is mainly based on Ref. [WWAF06, HWM ${ }^{+} 11$ ] and is very important since the KPM will be intensively used in this thesis.

\section{Rescaling scheme}

As mentioned above, a Chebyshev expansion only converges in the interval $[-1,1]$. Thus, in order to expand a zero-temperature spectral function in Chebyshev polynomials, one needs to map the full many-body bandwidth $W^{H}$ of the Hamiltonian $H$ to the interval $I \in[-1,1]$. In principle, any one-to-one mapping works for this purpose. However, one requirement is that the mapping has to be efficiently applied in the framework of matrix product states and operators since this operation has to be performed in each recursion step of a Chebyshev expansion. In this work, the standard choice, a linear rescaling scheme, used both in the KPM [WWAF06] and most MPS approaches $\left[\mathrm{HWM}^{+} 11, \mathrm{BS} 14\right.$, WMPS14] is adopted.

At zero temperature, one needs to rescale and shift the Hamiltonian $H$ for which $W^{H}=$ $E_{\max }^{H}-E_{\min }^{H}$. Here $E_{\min }^{H}$ denotes the ground-state energy and $E_{\max }^{H}$ the maximal energy

\footnotetext{
${ }^{26}$ Although in Ref. [Boy89] these results have been obtained for expansions using the unweighed scalar product in Eq. (3.69), they also hold for the scalar product in Eq. (3.73) [WJMS15].
} 
of the Hamiltonian $H$. In the following, we focus on $\omega>0$ and therefore consider $\omega \in\left[0, W^{H}\right]{ }^{27}$ In this case the linear map for the frequency reads

$$
\begin{aligned}
\omega \in\left[0, W^{H}\right] & \rightarrow \omega^{\prime} \in\left[-W^{\prime}, W^{\prime}\right], \quad W^{\prime}=1-\epsilon / 2, \\
\omega & \mapsto \omega^{\prime}=\frac{\omega}{a}-W^{\prime}, \quad a=\frac{W^{H}}{2 W^{\prime}} .
\end{aligned}
$$

Accordingly, the Hamiltonian is shifted and rescaled by

$$
H^{\prime} \mapsto \frac{H-E_{0}}{a}-W^{\prime}
$$

such that $H^{\prime}$ possesses the ground-state energy $E_{0}^{\prime}=W^{\prime}$. The choice of $W^{\prime}<1$ with $\epsilon=0.025$ acts as a safeguard to strictly impose $\omega^{\prime} \in[-1,1]$ in order to exclude divergences due to numerical inaccuracies. Note that the support of the zero-temperature spectral functions calculated in this thesis lies within the lower part of the interval $\left[0, W^{H}\right]$, which is mapped to the lower part of the interval $\left[-W^{\prime}, W^{\prime}\right]$ by the linear rescaling scheme.

\section{Operator-valued Chebyshev expansion}

Zero-temperature spectral functions with respect to the operators $B$ and $C$ are of the form

$$
R_{B C}^{T=0}(\omega)=\left\langle E_{0}\left|B \delta\left(\omega-H+E_{0}\right) C\right| E_{0}\right\rangle
$$

See Sec. 2.2.3 for the derivation. After applying the linear rescaling scheme in Eqs. (3.85) and (3.86), the spectral function is given by

$$
R_{B C}^{T=0}(\omega)=\frac{1}{a}\left\langle E_{0}\left|B \delta\left(\omega^{\prime}-H^{\prime}\right) C\right| E_{0}\right\rangle .
$$

The operator-valued expression $f(x)=\delta\left(x-H^{\prime}\right)$ with $x=\omega^{\prime}$ is now expanded in Chebyshev polynomials. One formally uses the modified moments in Eq. (3.73) and the truncated series in Eq. (3.78) in order to obtain an operator-valued Chebyshev expansion by means of the KPM:

$$
\delta\left(\omega^{\prime}-H^{\prime}\right) \approx \frac{1}{\pi \sqrt{1-\omega^{\prime 2}}}\left[g_{0} \mu_{0}+2 \sum_{n=1}^{N-1} g_{n} T_{n}\left(H^{\prime}\right) T_{n}\left(\omega^{\prime}\right)\right] .
$$

Inserting this result into Eq. (3.88) gives the Chebyshev expansion of the spectral function:

$$
R_{B C}^{T=0} \approx \frac{1}{\pi a \sqrt{1-\omega^{\prime 2}}}\left[g_{0} \mu_{0}+2 \sum_{n=1}^{N-1} g_{n} \mu_{n} T_{n}\left(\omega^{\prime}\right)\right] .
$$

Note that this expression includes the damping factors $g_{n}$ which lead to a broadened spectral function as already discussed in Sec. 3.5.3. The Chebyshev moments

$$
\mu_{n}=\left\langle E_{0}\left|B T_{n}\left(H^{\prime}\right) C\right| E_{0}\right\rangle
$$

\footnotetext{
${ }^{27}$ This is a consequence of the shifted delta functions $\delta\left(\omega-\left(E_{n}-E_{0}\right)\right)$ appearing in the Lehmann representation of the zero-temperature spectral function.
} 
turn out to be given by a ground-state expectation value of essentially an $n$th order Chebyshev polynomial in $H^{\prime}$. By defining the $n$th Chebyshev vector $\left|t_{n}\right\rangle$, the moments can be stated as

$$
\mu_{n}=\left\langle E_{0}|B| t_{n}\right\rangle, \quad\left|t_{n}\right\rangle=T_{n}\left(H^{\prime}\right) C\left|E_{0}\right\rangle .
$$

Now the main task is to determine $\left|t_{n}\right\rangle$, which is possible via the Chebyshev recursion in Eq. (3.67). One starts with the ground state and obtains $\left|t_{0}\right\rangle$ after applying the operator of interest to it. Then Eq. (3.67) translates into

$$
\left|t_{n}\right\rangle=2 H^{\prime}\left|t_{n-1}\right\rangle-\left|t_{n-2}\right\rangle, \quad\left|t_{1}\right\rangle=H^{\prime}\left|t_{0}\right\rangle, \quad\left|t_{0}\right\rangle=C\left|E_{0}\right\rangle .
$$

In the original KPM [WWAF06] the operations in this recursion relation are implemented by means of operations commonly used for the exact diagonalization of sparse Hamiltonian matrices, i.e., matrix-vector and vector-vector operations, as the $\left|t_{n}\right\rangle$ are represented by vectors. In order to treat larger systems, the MPS implementation of Eq. (3.93) is reviewed in the next section.

\section{MPS evaluation of the Chebyshev moments}

First of all, the ground state $\left|E_{0}\right\rangle$ is calculated as explained in Sec.3.3.3. Moreover, the rescaled Hamiltonian $H^{\prime}$ is represented as an MPO and the Chebyshev vectors will be represented as MPS. ${ }^{28}$ The recursion relation in Eq. (3.93) is implemented using the variational compression reviewed in Sec. 3.3.4. Given a maximal internal dimension $m$ of the MPS matrices, the state $\left|t_{n}\right\rangle$ is found via variationally minimizing the quantity

$$
\epsilon_{\mathrm{compr}}=\|\left|t_{n}\right\rangle-\left(2 H^{\prime}\left|t_{n-1}\right\rangle-\left|t_{n-2}\right\rangle\right) \|
$$

by sweeping through the chain. The sweeps are stopped once $\epsilon_{\text {compr }}$ is sufficiently small. The accuracy of the calculations is controlled by specifying $m$. For a zero-temperature calculation, this compression error is usually well controlled for the first $\sim 1000$ recursion steps, i.e., at most $\epsilon_{\text {compr }} \sim 10^{-5}$ for $m \sim 100$. The recursion represents the main and computationally most time consuming part of the method. It usually makes up about 80-90\% of the computation time.

\section{Resolution}

An important consequence of the rescaling scheme is that the resolution is modified. In the case of Gaussian broadening as in Eq. (3.81) and a linear map, the resolution of the Chebyshev-expanded spectral function is given by

$$
\eta(\omega, W)=\frac{\pi}{N} \frac{W}{2 W^{\prime}} \sqrt{1-\omega^{\prime 2}}
$$

As noted before, the resolution is generally not uniform as it does not only depend on the expansion order $N$ and the bandwidth $W$ but also on the frequency. Remember that the support of the zero-temperature spectral function is mapped onto the lower part of the interval $\left[-W^{\prime}, W^{\prime}\right]$, where $\omega^{\prime}$ is only slightly larger than -1 . Therefore, the broadening varies significantly in this frequency range. However, this in fact represents an advantage as the resolution is enhanced towards small frequency $\omega$. In many plots it is desirable to show the spectral function using a uniform broadening at all frequencies, the expansion order is thus adapted as a function of the frequency, if not stated otherwise.

\footnotetext{
${ }^{28}$ Note that the term Chebyshev vector is still used although this state is represented as an MPS.
} 


\section{Developments for increased resolution}

As a linear rescaling scheme with respect to the full spectral width provides a more or less uniform resolution, ${ }^{29}$ one may wonder whether it is possible to enhance the resolution, e.g., on a particular subinterval, where the physical features of interest are found $\left[\mathrm{HWM}^{+} 11\right]$. After the introduction of MPS-based Chebyshev expansions, there have been proposals $\left[\mathrm{HWM}^{+} 11, \mathrm{GTV}^{+} 14, \mathrm{WJMS} 15\right]$ of how to enhance the spectral resolution.

Two of these proposals are based on modifications of the rescaling scheme. The first approach $\left[\mathrm{HWM}^{+} 11\right]$ proposes to work with an effective band width instead of mapping the entire bandwidth to $[-1,1]$. The smaller interval can be chosen comparable to the actual support of the spectral function. The procedure is known as high-energy truncation and removes the contributions from higher frequencies $\left|\omega^{\prime}\right|>1$ contained in the Chebyshev vectors. This is done in a DMRG-like sweeping procedure and is absolutely necessary to avoid divergencies when working with this smaller interval. However, high-energy truncation cannot be formulated variationally such as the ground-state DMRG algorithm. Hence, there is no notion of optimality with respect to a certain measure and no simple criterion for convergence $\left[\mathrm{HWM}^{+} 11, \mathrm{GTV}^{+} 14\right]$. This drawback complicates the error analysis significantly because the calculations have to be performed for several different energy truncation parameters to assess their accuracy, which counteracts the possible speedup. In addition, recent results also suggested that a gain in resolution with respect to a fixed amount of computational resources cannot be expected [BS14, WMPS14]. There are also hints that high-energy truncation leads to an unfavorable increase in entanglement for the Chebyshev vectors $\left[\mathrm{HWM}^{+} 11\right]$, i.e., a slower decay of the eigenvalues of the corresponding reduced density matrices. For these reasons, a simple linear rescaling scheme with respect to the full width of the spectrum is used in this thesis.

The second approach $\left[\mathrm{GTV}^{+} 14\right]$ proposed to enhance the resolution of MPS-based Chebyshev expansions at $T=0$ advocates the map $H^{\prime}=I-\exp \left(-\tau\left(H-E_{0}+\epsilon\right)\right)$ using a sufficiently small $\tau$. Here $1 / \tau$ plays a similar role as the rescaling parameter $a$ in Eq. (3.85). Moreover, the map is convenient since such an exponential can be applied to MPS by efficient time-evolution algorithms, see Sec. 3.4. This rescaling scheme gives the highest resolution for the part of the spectrum located at low frequency in $\left[0, W^{H}\right]$. At $T=0$, this subinterval corresponds to the support of the spectral function. However, at $T>0$, the support does not lie close to this region (cf. Sec. 3.6.4). Therefore, the exponential rescaling scheme as presented in Ref. $\left[\mathrm{GTV}^{+} 14\right]$ is inconvenient for finite-temperature calculations and the linear scheme using the full bandwidth gives a better resolution.

The other two proposals for enhancing the resolution aim at improving the convergence properties of the Chebyshev moments in certain cases [GTV ${ }^{+} 14$, WJMS15]. The basic idea behind this is the removal of jumps in the spectral functions, thereby creating a faster convergence of the Chebyshev moments (see Sec. 3.5.3). Then the damping factors introduced to cure Gibbs oscillations in the KPM can be discarded, allowing for a higher resolution.

The idea has been applied to the zero-temperature spectral function of $1 \mathrm{D}$ fermionic models. If one considers the particle $A^{>}(\omega>0)$ or hole branch $A^{<}(\omega<0)$ of the spectral function separately, each of them may assume a finite value $A^{>}(0)>0\left[A^{<}(0)>0\right]$ at $\omega=0$ observed as a step at the Fermi energy $\omega=0$. Hence, there occurs a jump if one calculates each branch by an individual Chebyshev expansion leading to an algebraic decay

\footnotetext{
${ }^{29}$ Note that the resolution towards the end points of the interval is higher, but generally this statement is true for the central region.
} 
of the moments. By expanding the particle $A^{>}(\omega>0)$ and hole branches $A^{<}(\omega<0)$ as the full spectral function $A(\omega)=A^{>}(\omega)+A^{<}(-\omega)$, such a jump occurring at $\omega=0$ is removed. This has been shown to lead to an exponential decay of Chebyshev moments for the noninteracting single-impurity Anderson model which allows for a reliable extrapolation of the moments by linear prediction $\left[\mathrm{GTV}^{+} 14\right]$. The moments are extrapolated until they are negligibly small allowing for a truncation of the Chebyshev expansion without Gibbs oscillations and without the need of introducing broadening in such cases. The technique of linear prediction will be discussed in the next section.

Alternatively, it is also possible to do an individual Chebyshev expansion of one single branch $A^{>}(\omega>0)\left[A^{<}(\omega<0)\right]$ [WJMS15]. This is achieved by subtracting the Chebyshev expansion of a step function of finite height $A^{>}(0)\left[A^{<}(0)\right]$ in a self-consistency loop using linear prediction in each step.

\section{Linear prediction}

In the DMRG context, linear prediction [Mak75] has originally been used to extrapolate real-time evolution data both at zero [WA08] and finite temperature [BSW09]. For timedependent DMRG, it therefore is an established method, but recently it has also been applied to extrapolate Chebyshev moments $\mu_{n}\left[\mathrm{GTV}^{+} 14\right.$, WJMS15]. In Chapter 7, it will be used to predict the Chebyshev moments of finite-temperature spectral functions.

In a more general context, linear prediction is an approach to extrapolate the behavior of a sequence $x_{0}, x_{1}, \ldots, x_{N}$ of equidistant data points. These sequences may contain oscillations, but the method works best for exponentially decaying envelopes of the data. As the name might suggest, it makes the assumption that each predicted data point $\tilde{x}_{n}$ with $n>N$ is approximated by a linear combination of the previous $p$ data points:

$$
\tilde{x}_{n} \approx-\sum_{j=1}^{p} a_{j} x_{n-j} .
$$

The coefficients $a_{j}$ are determined by solving a minimization problem for the least-squares error given by

$$
F=\sum_{n \in I_{\mathrm{fit}}} F_{n}=\sum_{n \in I_{\mathrm{fit}}}\left(\tilde{x}_{n}-x_{n}\right)^{2}
$$

on a fitting subset $I_{\text {fit }}$ containing all $n \in\left\{N-N_{\text {fit }}-1, \ldots, N-1, N\right\}$. Differentiation with respect to a fixed $n$ leads to

$$
\frac{\partial F_{n}}{\partial a_{i}}=2\left(-\sum_{j=1}^{p} a_{j} x_{n-j}-x_{n}\right) \cdot\left(-x_{n-i}\right) .
$$

The full expression $\left(\partial F / \partial a_{i}\right)$ set to zero then yields

$$
\sum_{j=1}^{p}[\underbrace{\left(\sum_{n \in I_{\text {fit }}} x_{n-i} x_{n-j}\right)}_{=: R_{i j}} a_{j}]=-\underbrace{\sum_{n \in I_{\text {fit }}} x_{n} x_{n-i}}_{=: r_{i}},
$$

which with the above definitions can be identified as one system of equations

$$
R \cdot a=-r
$$




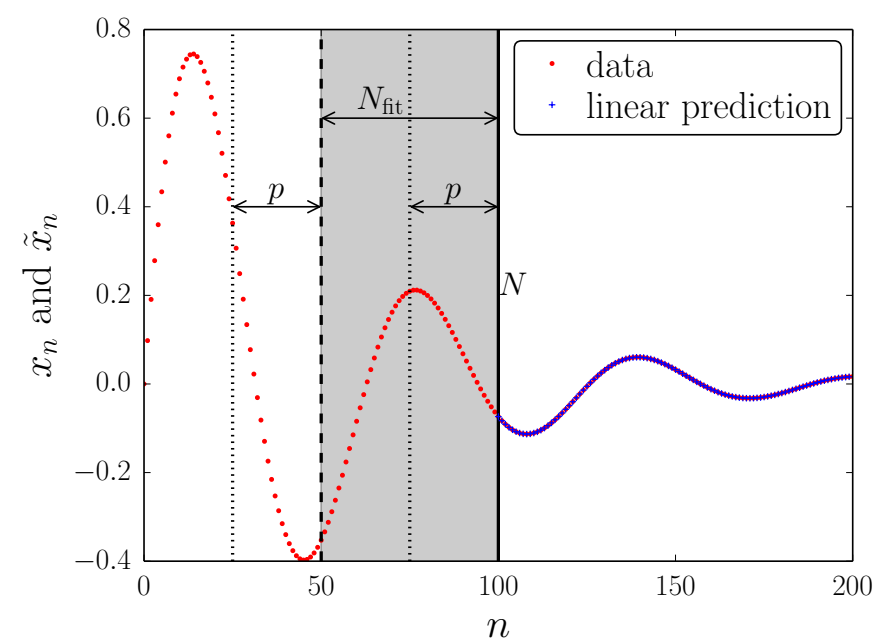

Figure 3.6: Assuming that only the first $N=100$ data points are known, the extrapolation of the sequence $x_{n}=\sin (n / 10) \cdot \mathrm{e}^{-0.02 n}$ by linear prediction is shown for illustrative purposes. The fitting interval consists of $N_{\text {fit }}$ data points (shaded region). Note that also the first of them is obtained by a linear combination of the previous $p$ data points. Therefore, also these data are needed in order to determine the coefficients $a_{j}$ for the linear prediction. In this example the predicted points have an accuracy close to machine precision.

The solution for the coefficients is thus given by the vector $a=-R^{-1} \cdot r$. It has been found that $N_{\text {fit }}=N / 4$ is generally a sufficient size of the fitting interval $I_{\text {fit }}$ [WJMS15]. ${ }^{30}$ Moreover, $p$ should not be chosen too large in order to avoid overfitting. Hence, the choice $p=\min \left(N_{\text {fit }} / 2,100\right)$ is adopted [BSW09]. For the sake of numerical stability, one adds a small constant to the diagonal elements of the matrix $R$ prior to inverting it. ${ }^{31}$ Then the matrix $M$ defined by

$$
M=\left(\begin{array}{ccccc}
-a_{1} & -a_{2} & -a_{3} & \cdots & -a_{p} \\
1 & 0 & 0 & \cdots & 0 \\
\vdots & \ddots & \ddots & \ddots & \vdots \\
0 & 0 & \cdots & 1 & 0
\end{array}\right)
$$

can be used to recursively generate the predicted data points

$$
\tilde{x}_{N+k}=\left[M^{k} \cdot x_{N}\right]_{1} .
$$

The extrapolation of a sequence by linear prediction is illustrated in Fig. 3.6. However, the matrix $M$ may contain eigenvalues $\lambda_{i}$ with $\left|\lambda_{i}\right|>1$ which will lead to a divergence of the predicted data. In order to ensure convergence, $M$ is diagonalized and such eigenvalues are normalized, i.e., set to $\lambda_{i} /\left|\lambda_{i}\right|$. This is done prior to the calculation in Eq. (3.102). On the other hand, if the weight associated with the eigenvalues $\left|\lambda_{i}\right|>1$ makes up more than a few percent, it is plausible to conclude that the problem cannot be treated by linear prediction [WJMS15].

\footnotetext{
${ }^{30}$ This is the case since the sequence is not stochastic.

${ }^{31}$ Alternatively, it is also possible to work with a pseudo-inverse determined by an SVD with cutoff of the order of $10^{-6}$ for small singular values $\left[\mathrm{GTV}^{+} 14\right]$.
} 



\section{MPS frequency-domain methods at finite temperature}

Note that a much shorter version of the present section has been published as a Rapid Communication in Physical Review B [TMPH14] together with the proof-of-principle calculations shown in Chapter 5. Moreover, the content of Sec. 3.6.3 has been published as part of a Regular Article in Physical Review B $\left[\mathrm{THP}^{+} 16\right]$. Accordingly, some parts of the present section may resemble both manuscripts which were mainly written by me.

\section{Introduction $\quad 3.6 .1$}

Having reviewed MPS methods generating expansions of spectral functions at $T=0$ in Sec. 3.5, it is now possible to move on to the calculation of finite-temperature response functions in the frequency domain. The MPS formulation of frequency-domain dynamics at $T>0$ is one of the main objectives of this thesis. The idea is to devise a new finitetemperature approach by combining the purification of a mixed-state density operator ${ }^{32}$ at $T>0$ with the MPS frequency-domain methods known from $T=0$.

The method development is motivated by the observation that the resolution of lowfrequency properties in spectral functions can be rather limited if they are calculated by DMRG or MPS real-time evolution. This is the case as the properties at small frequency depend on the hardly accessible long-time behavior of response functions. As already discussed in Sec.3.4.1, the maximal accessible time is restricted by the growth of entanglement in the course of the time evolution.

The following sections are structured in the following way: First, in Sec. 3.6.2 the purification of mixed-state density operators is considered in the MPS context. Secondly, the dynamics of the purification are reviewed in Liouville space in Sec. 3.6.3. Moreover, the MPS-based Chebyshev expansion of finite-temperature spectral functions is formulated in Liouville space in Sec. 3.6.4. Last but not least, the MPS Lanczos method generating a CFE is also presented using the Liouvillian formulation of the dynamics in Sec. 3.6.5.

\section{\begin{tabular}{l|l} 
Purification & 3.6 .2
\end{tabular}}

A mixed-state density operator representing thermal states at $T>0$ can be purified. The resulting pure-state wave function $|\Psi\rangle$ is known as a thermofield state defined in a doubled Hilbert space. See Sec. 2.4 for details. In the DMRG context $|\Psi\rangle$ is mostly referred to as purification. The doubled Hilbert space is the tensor product space $\mathcal{H}_{P} \otimes \mathcal{H}_{Q}$ of the physical state space $\mathcal{H}_{P}$ and an auxiliary space $\mathcal{H}_{Q}$ chosen to be isomorphic to $\mathcal{H}_{P}$. The purified state $|\Psi\rangle$ can then be represented as an MPS. Alternatively, the doubled degrees of freedom can also be implemented by an MPO [Bar13, PEAT14] as the purification represented as an MPS in a doubled Hilbert space and the corresponding MPO can be mapped onto each other by an isomorphism. In this thesis, the MPS purification is used. The following paragraphs contain its definition on a lattice and other technical details and is mainly based on Ref. [Sch11].

\footnotetext{
${ }^{32}$ As obtained in thermofield dynamics (TFD), see Sec. 2.4.
} 
(a)

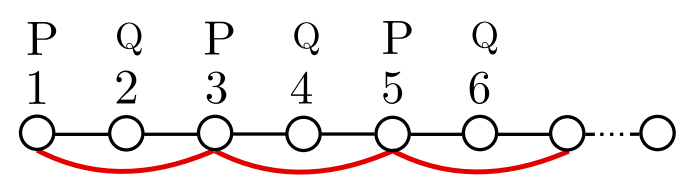

(b)

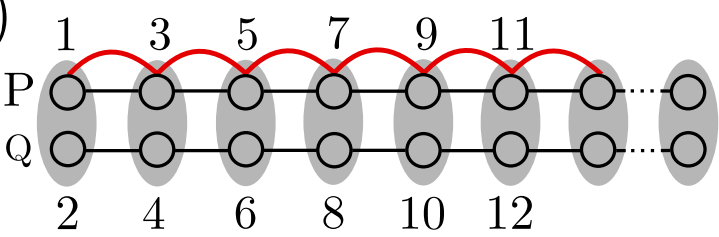

Figure 3.7: Purification of mixed states for a 1D system: (a) The physical degrees of freedom living in $\mathcal{H}_{P}$ reside at the odd sites of the lattice (marked by $\mathrm{P}$ ). The auxiliary degrees of freedom belonging to $\mathcal{H}_{Q}$ are situated at the even sites $(\mathrm{Q})$ between the physical sites in order to minimize the range of the physical nearest-neighbor interactions (red links) in the combined system. (b) Geometrically, this chain may also be thought of as a ladder with interactions between the physical sites (red links). The filled gray ellipses illustrate the maximally entangled state at each rung of the ladder at $T=\infty$.

In practical applications, the auxiliary state space is simply taken as a copy of the physical space, i.e., $\mathcal{H}_{Q}=\mathcal{H}_{P}$. As a consequence, the number of sites has to be doubled. To avoid longer-ranged interactions the physical and auxiliary sites are arranged in an alternating fashion as depicted in Fig. 3.7. This turns nearest-neighbor interactions between physical sites into next-nearest-neighbor interactions if the purification is considered. However, these slightly longer-ranged interactions can be treated by increasing the bond dimensions of the MPO representing the Hamiltonian $H_{P} \otimes I_{Q}$ for the purification $|\Psi\rangle$. Here the Hamiltonian $H$ and the identity operator $I$ act on the spaces specified by the respective indices.

As a next step, let us consider the density operator of the physical system, which can be obtained by tracing out the auxiliary degrees of freedom:

$$
\rho_{P}=\operatorname{Tr}_{Q}|\Psi\rangle\langle\Psi|
$$

Note that in the following the normalized thermal density operator at temperature $T$ is considered which is given by

$$
\begin{aligned}
\rho_{T} & =Z(T)^{-1} \mathrm{e}^{-\left(H_{P} \otimes I_{Q}\right) /\left(k_{\mathrm{B}} T\right)} \\
& =Z(T)^{-1} \mathrm{e}^{-\left(H_{P} \otimes I_{Q}\right) /\left(2 k_{\mathrm{B}} T\right)} \cdot I \cdot \mathrm{e}^{-\left(H_{P} \otimes I_{Q}\right) /\left(2 k_{\mathrm{B}} T\right)},
\end{aligned}
$$

where the inverse of $Z(T)=\operatorname{Tr}_{Q} \mathrm{e}^{-\left(H_{P} \otimes I_{Q}\right) /\left(k_{\mathrm{B}} T\right)}$ is used for the normalization. Note that the identity in Eq. (3.105) can be written as $I=Z(\infty) \rho_{\infty}$. With this replacement one obtains

$$
\rho_{T}=\frac{Z(\infty)}{Z(T)} \operatorname{Tr}_{Q} \mathrm{e}^{-\left(H_{P} \otimes I_{Q}\right) /\left(2 k_{\mathrm{B}} T\right)}\left|\Psi_{\infty}\right\rangle\left\langle\Psi_{\infty}\right| \mathrm{e}^{-\left(H_{P} \otimes I_{Q}\right) /\left(2 k_{\mathrm{B}} T\right)}
$$

as the Hamiltonian $H_{P} \otimes I_{Q}$ does not act on $\mathcal{H}_{Q}$. By comparing this result to Eq. (3.103), the purification of the thermal state can be identified as

$$
\left|\Psi_{T}\right\rangle=\mathrm{e}^{-\left(H_{P} \otimes I_{Q}\right) /\left(2 k_{\mathrm{B}} T\right)}\left|\Psi_{\infty}\right\rangle .
$$

Also note that $Z(T) / Z(\infty)=\left\langle\Psi_{T} \mid \Psi_{T}\right\rangle$. Since an infinite-temperature purification $\left|\Psi_{\infty}\right\rangle$ can be stated analytically, the above expression implies that $\left|\Psi_{T}\right\rangle$ is accessible via an imaginary-time evolution starting at $T=\infty$. The imaginary-time evolution successively 
lowers the temperature and represents a standard numerical tool (see Sec. 3.4). A purification at $T=\infty$ is a product state of maximally entangled states between the physical and auxiliary degrees of freedom at each rung of the ladder [Sch11]. This is depicted in Fig. 3.7 and assumes the following form

$$
\left|\Psi_{\infty}\right\rangle=\otimes_{j=1}^{L}\left|\Psi_{\infty, j}\right\rangle, \quad\left|\Psi_{\infty, j}\right\rangle=\frac{1}{\sqrt{d}} \sum_{s_{j}}\left|s_{j}\right\rangle_{P} \otimes\left|s_{j}\right\rangle_{Q}
$$

In the case of a spin-1/2 Heisenberg chain (see Sec. 4.2), another possible choice that is used in this thesis is the singlet state at each rung

$$
\left|\Psi_{\infty, j}\right\rangle=\frac{1}{\sqrt{2}}\left[\left|\uparrow_{P} \downarrow_{Q}\right\rangle-\left|\downarrow_{P} \uparrow_{Q}\right\rangle\right] .
$$

The corresponding MPS matrices for $j=1,2, \ldots, L$ are, e.g., given by

$$
M^{\uparrow_{2 j-1}}=\left(\begin{array}{ll}
1 & 0
\end{array}\right), \quad M^{\downarrow_{2 j-1}}=\left(\begin{array}{ll}
0 & -1
\end{array}\right), \quad M^{\uparrow_{2 j}}=\left(\begin{array}{ll}
0 & 1 / \sqrt{2}
\end{array}\right)^{T}, \quad M^{\downarrow_{2 j}}=\left(\begin{array}{ll}
1 / \sqrt{2} & 0
\end{array}\right)^{T} .
$$

This state conserves, e.g., the $z$ component of the total spin of the enlarged system consisting of both physical and auxiliary sites. However, this symmetry is broken for the Dzyaloshinskii-Moriya interactions effectively described by a transverse staggered field (see Chapter 4.5) and hence it is not possible to exploit it for the computations.

In the presence of symmetries, the authors of Ref. [NA16] reported an approach to further restrict the quantum numbers in the preparation of the purification. The very recent development proposes a computation within a subspace in which, e.g., the $z$ component of the total spin of the physical system is conserved for a Heisenberg chain. One consequently works in the canonical ensemble for the physical chain resulting in smaller Hilbert space dimensions. In contrast, a purification of the form (3.110) is used throughout this work. It leads to a calculation which can be considered as grand canonical.

In the course of the imaginary-time evolution starting at $T=\infty$, static thermodynamic properties can be calculated conveniently since the evaluation of the expectation value of an operator $A=A_{P} \otimes I_{Q}$ is given by

$$
\langle A\rangle_{T}=\operatorname{Tr}_{Q}\left(A \rho_{T}\right)=\frac{\left\langle\Psi_{T}\left|\left(A_{P} \otimes I_{Q}\right)\right| \Psi_{T}\right\rangle}{\left\langle\Psi_{T} \mid \Psi_{T}\right\rangle}
$$

The MPS-based evaluation of such expectation values can be performed with the same procedures used for zero-temperature expectation values. For instance, the internal energy $\langle H\rangle_{T}$ and $\left\langle H^{2}\right\rangle_{T}$ can be calculated in this manner, which will be used to evaluate the specific heat in Sec. 5.2.

\section{\begin{tabular}{l|l} 
Dynamics of the purification & 3.6 .3
\end{tabular}}

The dynamics of the purification also known as a thermofield state has been considered in Sec. 2.4. Here one may start by recapitulating a few things. If one thinks of the density operator $\rho$ as a state vector $|\rho\rangle\rangle$ in the Liouville space of operators, the corresponding Liouville-von Neumann equation in Eq. (2.44) becomes formally isomorphic to the timedependent Schrödinger equation in Hilbert space. According to Ref. [BD87], each vector 
$|\rho\rangle\rangle$ can be identified with a pure-state wave function $|\Psi\rangle$ in a doubled Hilbert space $\mathcal{H}_{P} \otimes \mathcal{H}_{Q}$. With this association, the Liouville operator $\mathcal{L}=H_{P} \otimes I_{Q}-I_{P} \otimes H_{Q}$ serves as the Hamiltonian for the purification $|\Psi\rangle$. Technically, $\mathcal{L}$ is implemented as an MPO. If one wants to study magnetic fields, it has to be noted that the fields included in $H_{Q}$ need to be reversed in sign due to time reversal symmetry [KBM13].

The eigenvalues of the operator $\mathcal{L}$ are the differences of the eigenenergies of the Hamiltonian $H$. From this, it becomes evident that a Liouville-space formulation is natural for the treatment of finite-temperature dynamics and the expression for a spectral function at $T>0$ is

$$
\begin{aligned}
A_{B C}^{T>0}(\omega)= & \frac{1}{Z} \sum_{n, m} \mathrm{e}^{-E_{n} /\left(k_{\mathrm{B}} T\right)}\left\langle E_{m}|B| E_{n}\right\rangle \times \\
& \times\left\langle E_{n}|C| E_{m}\right\rangle \delta\left(\omega-\left(E_{m}-E_{n}\right)\right) \\
= & \left\langle\Psi_{T}\left|\left(B_{P} \otimes I_{Q}\right) \delta(\omega-\mathcal{L})\left(C_{P} \otimes I_{Q}\right)\right| \Psi_{T}\right\rangle .
\end{aligned}
$$

See Sec. 2.5 for more details. Here $\left|\Psi_{T}\right\rangle \in \mathcal{H}_{P} \otimes \mathcal{H}_{Q}$ denotes the thermal state which is obtained via an imaginary-time evolution as explained in Sec. 3.6.2. The Liouvillian formulation can be used to recast finite-temperature spectral functions (see Eq. (3.112b)) into a form very similar to the $T=0$ expression in Eq. (3.87). Thus, standard numerical methods working directly in the frequency domain are inherently applicable also at $T>0$. Examples are a continued fraction expansion as well as a Chebyshev expansion of spectral functions. How these methods can be formulated in Liouville space is discussed in the following two sections.

\section{Chebyshev expansion of finite-temperature spectral functions}

\section{Rescaling scheme and resolution at $T>0$}

At finite temperature, the Chebyshev recursion is performed with respect to the Liouville operator $\mathcal{L}$ which also needs to be rescaled. To this end, it is helpful to realize that the eigenenergies of $\mathcal{L}$ are the differences of the eigenenergies of the Hamiltonian, the width of the spectrum of the Liouville operator turns out to be

$$
W^{\mathcal{L}}=E_{\max }^{\mathcal{L}}-E_{\text {min }}^{\mathcal{L}}=\left(E_{\text {max }}^{H}-E_{\text {min }}^{H}\right)-\left(E_{\text {min }}^{H}-E_{\text {max }}^{H}\right)=2 W^{H} .
$$

It hence assumes twice the value of the Hamiltonian width $W^{H}$. The linear rescaling scheme used in this thesis is given by the map

$$
\begin{aligned}
\omega \in\left[-\frac{W^{\mathcal{L}}}{2}, \frac{W^{\mathcal{L}}}{2}\right] & \rightarrow \omega^{\prime} \in\left[-W^{\prime}, W^{\prime}\right], \quad W^{\prime}=1-\epsilon / 2, \\
\omega & \mapsto \omega^{\prime}=\frac{1}{a}\left(\omega+\frac{W^{\mathcal{L}}}{2}\right)-W^{\prime}, \quad a=\frac{W^{\mathcal{L}}}{2 W^{\prime}} .
\end{aligned}
$$

The rescaled Liouvillian is

$$
\mathcal{L}^{\prime} \mapsto \frac{1}{a}\left(\mathcal{L}+\frac{W^{\mathcal{L}}}{2}\right)-W^{\prime}
$$

The support of a finite-temperature spectral function usually corresponds to the central region of $\left[-W^{\mathcal{L}} / 2, W^{\mathcal{L}} / 2\right]$, and is therefore mapped to rescaled frequencies $\omega^{\prime} \ll 1$ in the 
interval $\left[-W^{\prime}, W^{\prime}\right]$. This is one essential difference in comparison to $T=0$, where the support of the spectral function is rescaled to the lower part of the interval $\left[-W^{\prime}, W^{\prime}\right]$, i.e., $\omega^{\prime}$ is only slightly larger than -1 . Although Equation (3.95) for the broadening is still valid at $T>0$, there is only a weak frequency dependence in this case since the square-root expression in the equation is approximately one - at least as far as the support of the spectral function is concerned. The expansion order can hence be chosen constant at $T>0$ to obtain a nearly uniform resolution. However, the support mapped to $\omega^{\prime} \ll 1$ at $T>0$ also represents a drawback of the linear rescaling scheme since the resolution towards the boundaries of the interval $\left[-W^{\prime}, W^{\prime}\right]$ is higher. This is exploited at $T=0$. Furthermore, the spectral width of the Liouvillian assumes twice the width of the Hamiltonian, which reduces the resolution by a factor of two when working with the full bandwidth at $T>0$. These two issues make it computationally more expensive to obtain a high resolution at $T>0$.

\section{Operator-valued Chebyshev expansion at $T>0$}

Different from the Chebyshev expansion at $T=0$ described in Sec. 3.5.4, here an expansion in Chebyshev polynomials with respect to the linearly rescaled Liouville operator $\mathcal{L}^{\prime}$ from Eq. (3.114) is employed. Instead of the ground state, the thermal state $\left|\Psi_{T}\right\rangle$ is used as the initial state. It is represented as a pure-state wave function using the purification trick. This also applies to the other Chebyshev vectors generated by the recursion relation (3.93) which now translates into

$$
\left|t_{n}\right\rangle=2 \mathcal{L}^{\prime}\left|t_{n-1}\right\rangle-\left|t_{n-2}\right\rangle, \quad\left|t_{1}\right\rangle=\mathcal{L}^{\prime}\left|t_{0}\right\rangle, \quad\left|t_{0}\right\rangle=C\left|\Psi_{T}\right\rangle,
$$

where $C=C_{P} \otimes I_{Q}$ is written for brevity. The recursion represents the main and computationally most time consuming part of the method. The MPS evaluation of the Chebyshev moments $\mu_{n}$ proceeds along standard lines (see Sec. 3.5.4) using, for instance, variational compression. The coefficients

$$
\mu_{n}=\left\langle\Psi_{T}|B| t_{n}\right\rangle, \quad\left|t_{n}\right\rangle=T_{n}\left(\mathcal{L}^{\prime}\right) C\left|\Psi_{T}\right\rangle
$$

are computed after each recursion step. These coefficients are used to expand the finitetemperature spectral function in Eq. (3.112b) as

$$
A_{B C}^{T>0}(\omega) \approx \frac{1}{\pi a \sqrt{1-\omega^{\prime 2}}}\left[g_{0} \mu_{0}+2 \sum_{n=1}^{N-1} g_{n} \mu_{n} T_{n}\left(\omega^{\prime}\right)\right] .
$$

The example of MPS-based Chebyshev expansions at $T>0$ demonstrates how the calculation of finite-temperature spectral functions with frequency-domain methods known from $T=0$ can be formulated in Liouville space. Nevertheless, note that one has to pay a higher computational price than at $T=0$. One issue is the doubled system size needed for the purification. As illustrated in Fig. 3.7, physical nearest-neighbor interactions become next-nearest-neighbor interactions in the Liouville operator. ${ }^{33}$ Consequently, the internal bond dimensions $D$ of the MPO representing $\mathcal{L}^{\prime}$ are slightly larger than for the Hamiltonian. For a Heisenberg chain, one finds $D\left(H^{\prime}\right)=5$ and $D\left(\mathcal{L}^{\prime}\right)=8$ as an example.

\footnotetext{
${ }^{33}$ There are also next-nearest-neighbor interactions between auxiliary sites due to the term $-I_{P} \otimes H_{Q}$ in the Liouvillian.
} 


\section{Continued fraction expansion at $T>0 \quad 3.6 .5$}

Besides MPS-based Chebyshev expansions of finite-temperature spectral functions, it is also possible to formulate the MPS Lanczos method generating a CFE in Liouville space. This demonstrates the flexibility of the formulation. Consequently, the results are obtained by a continued fraction expansion $(\mathrm{CFE})$ of the spectral function generated by the Lanczos algorithm with respect to the Liouville operator $\mathcal{L}$ :

$$
\begin{aligned}
& \left|f_{i+1}\right\rangle=\mathcal{L}\left|f_{i}\right\rangle-a_{i}\left|f_{i}\right\rangle-b_{i}^{2}\left|f_{i-1}\right\rangle \quad\left(b_{0} \equiv 0,\left|f_{-1}\right\rangle \equiv 0\right) \\
& a_{i}=\left\langle f_{i}|\mathcal{L}| f_{i}\right\rangle /\left\langle f_{i} \mid f_{i}\right\rangle, \quad b_{i}^{2}=\left\langle f_{i} \mid f_{i}\right\rangle /\left\langle f_{i-1} \mid f_{i-1}\right\rangle
\end{aligned}
$$

Here the Lanczos states $\left|f_{i}\right\rangle$ are also represented by MPS purifications living in the tensor product space $\mathcal{H}_{P} \otimes \mathcal{H}_{Q}$. The initial state for the Lanczos recursion is

$$
\left|f_{0}\right\rangle=B\left|\Psi_{T}\right\rangle / \sqrt{\left\langle\Psi_{T}\left|B^{\dagger} B\right| \Psi_{T}\right\rangle},
$$

where $\left|\Psi_{T}\right\rangle$ still denotes the thermal state obtained via imaginary-time evolution. Having determined the coefficients from Eq. (3.118), a finite-temperature dynamical response function is approximated by a CFE of the form

$$
\begin{aligned}
S_{B^{\dagger} B}^{T>0}(z=\omega+i \eta) & =-\frac{1}{\pi} \operatorname{Im}\left\langle\Psi_{T}\left|B^{\dagger} \frac{1}{z-\mathcal{L}} B\right| \Psi_{T}\right\rangle \\
& \approx-\frac{1}{\pi} \operatorname{Im} \frac{\left\langle\Psi_{T}\left|B^{\dagger} B\right| \Psi_{T}\right\rangle}{z-a_{0}-\frac{b_{1}^{2}}{z-a_{1}-\frac{b_{2}^{2}}{z-a_{2}-\ldots}}} .
\end{aligned}
$$

Different from the MPS Chebyshev method, CFE results exhibit an intrinsic Lorentzian broadening both at $T=0$ and $T>0$. Depending on the number of Lanczos iterations $n$, the value $\eta$ of the broadening is chosen such that the spectral function is visibly converged. Essentially, the spectral function is approximated by a sum of Lorentzian peaks. This is different from Chebyshev expansions where highly oscillating polynomials are used. Thus, it might be harder to resolve, e.g, the multi-particle continua of Heisenberg chains with a CFE.

For a little more completeness, it has also to be noted that there are also finite-temperature variants of the Lanczos method which allow for the calculation of dynamical properties. These methods are not implemented using MPS or purifications but use a vector representation of the Lanczos states, as explained in Sec. 3.2. These finite-temperature methods are summarized in Ref. [PB13]. All of them are a combination of the Lanczos method and stochastic sampling. At low temperature, only a smaller amount of sampling is needed compared to higher temperatures. The most prominent method in this context is the finite-temperature Lanczos method (FTLM) [JcvPcv94, JP00] and its low-temperature version [ADEvdL03]. The FTLM has also been combined with the traditional DMRG. This method only works well at rather low temperatures $T / J<0.5$ as it is based on stochastic sampling. It can treat Heisenberg chains of length $L \leq 40$ [KPcv09]. 


\section{\begin{tabular}{l|l} 
Models & 4
\end{tabular} \\ \begin{tabular}{l|l} 
Introduction & 4.1
\end{tabular}}

This chapter is concerned with the review of models effectively describing the magnetic properties of quasi-1D materials. A typical solid is composed of ions and electrons that are condensed in a crystalline structure in three spatial dimensions. Even a small chunk of a solid having a volume of $1 \mathrm{~cm}^{3}$ contains a gigantic number of such constituents, i.e., of the order of $10^{23}$ particles. To describe such many-body quantum systems, it is necessary to reduce the very large number of variables by making justified approximations. This will finally lead to effective models allowing for the description of many quantum phenomena in solids. One very common phenomenological simplification is the decoupling of the dynamics of the atomic nuclei and the electrons. This assumption is known as the Born-Oppenheimer approximation [BH54]. The motion of the nuclei can be neglected and one may think of them as a static lattice. ${ }^{1}$ This is well justified since the nuclei are considerably heavier ${ }^{2}$ than the electrons which accordingly move significantly faster. Then the dynamics of the electrons are considered in the periodic potential of fixed ions. It is also possible to make a further drastic simplification such as neglecting the repulsive two-body interactions among electrons. However, this thesis is concerned with quantum magnetism and therefore strong correlations as introduced by the Coulomb interaction represent an indispensable ingredient.

A very important model for interacting electrons which displays true many-body phenomena driven by strong correlations is the Hubbard model [Gut63, Hub63, Kan63, EFG ${ }^{+}$05]. Since the major motivation behind this work is the study of quasi-1D materials, only 1D models are considered in the following. In the formalism of second quantization [Mah00, Pru14], the Hubbard Hamiltonian for a chain of atoms is given by

$$
H=H_{t}+H_{U}=-t \sum_{i, s}\left(c_{i, s}^{\dagger} c_{i+1, s}+c_{i+1, s}^{\dagger} c_{i, s}\right)+U \sum_{i} n_{i, \uparrow} n_{i, \downarrow} .
$$

Here $c_{i, s}^{(\dagger)}$ denotes the annihilation (creation) operator for an electron at site $i$ with spin $s=\uparrow, \downarrow$. At each site the local Hilbert space is spanned by the basis $\{|0\rangle,|\downarrow\rangle,|\uparrow\rangle,|\downarrow \uparrow\rangle\}$, where the last basis state corresponds to a doubly occupied site. Moreover, due to the Pauli exclusion principle double occupancies of two electrons with the same spin quantum number are forbidden.

The first term $H_{t}$ in Eq. (4.1) is responsible for the itinerant behavior of the electrons. However, here one assumes that the electrons are mainly localized at the lattice sites while they are allowed to hop between adjacent sites. ${ }^{3}$ This is called tight-binding approxima-

\footnotetext{
${ }^{1}$ This, e.g., includes neglecting phonons which provide a quantum mechanical description of vibrational lattice modes.

${ }^{2}$ About four orders of magnitude heavier.

${ }^{3}$ Here isotropic hopping amplitudes $t$ are assumed.
} 
tion. So there is only one orbital per site having a sufficient overlap with the orbitals at neighboring sites. This is a valid hypothesis for the derivation of models. However, in real materials consisting of magnetic ions with partially filled $d$ or $f$ orbitals, the situation is more subtle and will be discussed further below.

The second contribution $H_{U}$ to the Hubbard model incorporates the Coulomb interactions. However, this simple variant of the model assumes only local interactions. This is usually the case due to the strong screening in transition and rare earth metals. The Coulomb interactions are thus mimicked by a repulsive $(U>0)$ on-site interaction favoring a localization of the electrons. The model does hence not include long-range Coulomb forces. The particle number operator at site $i$ is denoted by $n_{i, s}=c_{i, s}^{\dagger} c_{i, s}$. The interplay of these two contributions determines the behavior of the system.

Although the above approximations have led to a simplified model in Eq. (4.1), there has so far been no general analytic treatment of it. However, it is possible to calculate the exact eigenvalues and eigenstates of the 1D Hubbard model via the Bethe ansatz [LW68], but even the calculation of the norm of the exact eigenfunctions poses a challenge [GK99]. Despite these difficulties, the Hubbard model plays an important role in the study of itinerant magnetism and metal-insulator transitions. These two aspects are for instance treated pedagogically in Ref. [Faz99]. There has been a lot of progress in understanding its physical properties by a combination of analytical and numerical approaches.

\section{\begin{tabular}{l|l} 
The Heisenberg model & 4.2
\end{tabular}}

Rather than reviewing the Hubbard model further, it is more important to point out a special case which is studied throughout this thesis. This is the strongly interacting limit $U / t \gg 1$ in combination with a half-filled electron band which sets the stage for quantum magnetism. Then every site is essentially singly occupied in order to avoid double occupancies which are energetically unfavorable in the presence of a strong electronic onsite repulsion. This gives rise to a localized magnetic moment carrying a spin of $S=1 / 2$ at each site. In this situation the system can be described by nearest-neighbor exchange interactions between the magnetic spins. This is known as the Heisenberg model, which has originally been proposed already back in 1928 [Hei28].

Exchange interactions are of quantum mechanical origin and occur between identical particles such as electrons. Generally, they give the main reason for spin ordering in magnetic materials leading to, e.g, antiferromagnetism which is obtained in the case of a predominantly alternating ordering of the electronic spins. Furthermore, ferromagnetism may emerge when the magnetic moments of electrons are mostly aligned. The classical dipolar interactions between the electronic spins are too weak (about $10^{-4} \mathrm{eV}$ corresponding to $1 \mathrm{~K}$ ) in order to account for such ordering since it is commonly observed at much higher temperatures. Exchange interactions arise due to the following fundamental properties of electrons [Aue94]:

- The electron's intrinsic angular momentum.

- Its kinetic delocalization energy.

- The Pauli exclusion principle.

- The Coulomb repulsion between electrons. 
In order to motivate the Heisenberg Hamiltonian in the next section, the exchange interaction between electronic spins at adjacent sites in the single-orbital Hubbard model is considered.

\section{\begin{tabular}{l|l} 
The Heisenberg Hamiltonian & 4.2 .1
\end{tabular}}

The following discussion is mainly based on Ref. [Faz99]. In order to obtain the Heisenberg Hamiltonian, one regards the Hubbard Hamiltonian (4.1) in the strongly interacting limit $U / t \gg 1$ and at half filling and resorts to perturbation theory. In the perturbative treatment $H_{U}$ represents the zeroth-order term and the weak $(t / U \ll 1)$ single-particle Hamiltonian $H_{t}$ is the perturbation. This is different from standard perturbation theory since one usually treats problems with a strong one-particle Hamiltonian and a weak interaction term. In such a standard case the zeroth-order ground state has no degeneracy. However, here the ground-state of the zeroth-order term $H_{U}$ is highly degenerate and the weak perturbation by $H_{t}$ has a considerable effect and lifts the degeneracy. It therefore mixes the degenerate eigenstates of $H_{U}$. In order to treat higher orders of $t / U$, a series of canonical transformations can be applied to derive an effective spin Hamiltonian. In a first step, a new eigenbasis is constructed from the zeroth-order eigenstates of $H_{U}$ whose elements are not mixed in order $t$. In a second step, mixing of order $t^{2}$ is avoided. A detailed discussion of the perturbative treatment can be found in Ref. [Faz99]. As a result, at half filling and $U / t \gg 1$ the spin-1/2 Heisenberg model effectively describes the lowenergy behavior of the repulsive Hubbard model. The isotropic ${ }^{4}$ Heisenberg Hamiltonian defined on a chain with $L$ sites is given by

$$
H=J \sum_{j=1}^{L-1} \mathbf{S}_{j} \cdot \mathbf{S}_{j+1}=\sum_{j=1}^{L-1} \frac{J}{2}\left(S_{j}^{+} S_{j+1}^{-}+S_{j}^{-} S_{j+1}^{+}\right)+J S_{j}^{z} S_{j+1}^{z} .
$$

It can be determined in second-order perturbation theory in $t$ and represents a paradigmatic spin model. Here $\mathbf{S}_{l}$ represents the spin operator at site $l$ with components $S^{\alpha}$ where $\alpha=x, y, z$. Heisenberg spins can be oriented in any spatial direction in three dimensions. Moreover, one has $S_{l}^{+}=S_{l}^{x}+i S_{l}^{y}$ and $S_{l}^{-}=S_{l}^{x}-i S_{l}^{y}$. There is an antiferromagnetic exchange coupling $J$ between nearest-neighbor spins. This can be understood by considering two adjacent antiparallel spins at different sites. Then a virtual hopping process can lead to, e.g., the following transition

$$
|\downarrow\rangle,|\uparrow\rangle \rightarrow|0\rangle,|\downarrow \uparrow\rangle
$$

resulting in an energy gain of $\sim t^{2} / U$ in second-order perturbation theory. The coupling between nearest-neighbor spin operators is given by $J=4 t^{2} / U$ in second order. Note that the coupling constant sets the global energy scale of the system. Having only considered antiparallel spin alignment, one finally has to note that for a parallel configuration of neighboring spins such a virtual process is excluded by the Pauli principle.

\footnotetext{
${ }^{4}$ There are also anisotropic variants of the Heisenberg model such as the $X X Z$ model with anisotropic exchange in the $z$ direction. Although it is not considered in this thesis, further generalizations of the isotropic Heisenberg model are reviewed in Ref. [MK04].
} 
The Heisenberg Hamiltonian acts on spin states having a basis $\{|\downarrow\rangle,|\uparrow\rangle\}$ at each site. Also note that the $x, y, z$ components of the quantum spin operators obey the commutation relations

$$
\left[S_{l}^{\alpha}, S_{l^{\prime}}^{\gamma}\right]=i \epsilon_{\alpha \gamma \nu} \delta_{l l^{\prime}} S_{l}^{\nu}
$$

where $\epsilon_{\alpha \gamma \nu}$ denotes the totally antisymmetric Levi-Civita tensor. Apart from the low dimensionality of the model, the noncommutativity in Eq. (4.4) leads to quantum behavior which is considerably enhanced for a small value of the spin, e.g., for $S=1 / 2$ treated here.

In the ground state of the 1D antiferromagnetic Heisenberg model, there is no long-range order due to the presence of strong quantum fluctuations. The ground state is a spin singlet and can be described by the massless Luttinger liquid theory [Gia04].

\section{Excitations $\mid 4.2 .2$}

The following paragraphs contain a brief discussion of the ground-state excitations in the 1D spin-1/2 Heisenberg antiferromagnet. For a more detailed cf. Refs. [Faz99, MK04]. Its elementary excitations are fractional spin- $1 / 2$ quasiparticles which are interacting weakly in a non-trivial manner. They are known as spinons. Assuming an infinite $1 \mathrm{D}$ chain with lattice periodicity $a$, the spinons have a dispersion relation [dCP62] given by

$$
\omega_{1}(q)=\frac{\pi}{2}|J \sin (a q)|, \quad q \in\left[-\frac{\pi}{a}, \frac{\pi}{a}\right]
$$

where $q$ represents the wave vector $(\hbar=1)$. The dispersion is depicted as the lower boundary of the two-spinon continuum in Fig. 4.1. Two-spinon excitations carry a spin of $S=1$. Their continuum governs the dynamics of the isotropic Heisenberg antiferromagnet. Its upper boundary is given by

$$
\omega_{2}(q)=\pi\left|J \sin \left(\frac{a q}{2}\right)\right| .
$$

Moreover, the boundaries of the two-spinon continua in the 1D Heisenberg antiferromagnet can be calculated exactly by Bethe ansatz in the presence of a uniform magnetic field [MTBB81].

The excitation spectrum is gapless at $q=0$ and $q= \pm \pi / a$. Although the 1D Heisenberg model can be solved exactly by Bethe ansatz [Bet31] which provides for instance the excitation energies and all eigenstates, the calculation of the dynamical spin structure factor also involves matrix elements of spin operators between excited states. This renders analytical calculations complicated. However, the contribution of both the two-spinon $\left[\mathrm{KMB}^{+} 97\right]$ and four-spinon excitations $[\mathrm{CH} 06]$ to the zero-temperature dynamical spin structure factor have been obtained exactly. These works showed, e.g., that at $T=0$ the two-spinon excitations contribute $72.89 \%$ to the total integrated intensity whereas the four-spinon contributions make up $27 \pm 1 \%$. Consequently, the remaining $\sim 1 \%$ of the intensity arise from higher spinon excitations [CH06].

\section{\begin{tabular}{l|l} 
Ferromagnetic Heisenberg model & 4.2 .3
\end{tabular}}

Originally, the Heisenberg model was proposed in 1928 [Hei28] to effectively describe both ferromagnetism and antiferromagnetism. In the case of ferromagnetic exchange couplings 


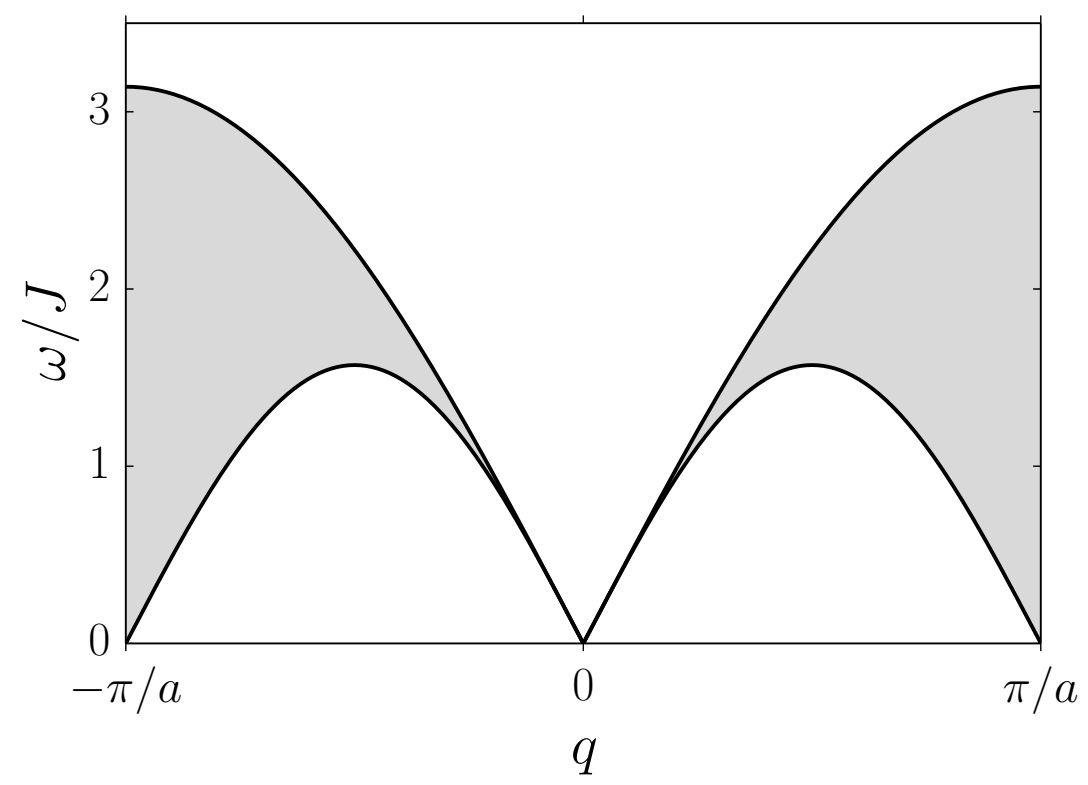

Figure 4.1: (a) Isotropic Heisenberg antiferromagnet: The spinon dispersion $\omega_{1}(q)$ from Eq. (4.5) represents the lower boundary of the two-spinon continuum whose extent is marked by the gray shaded region. Its upper boundary is given by $\omega_{2}(q)$ in Eq. (4.6)

$(J<0)$, the Heisenberg model in Eq. $(4.2)$ has a ground state in which the maximum value of the total spin is assumed. Therefore, the state

$$
|\uparrow \uparrow \ldots \uparrow\rangle
$$

is a ground state of the system which is maximally polarized in the $z$ direction. However, the Heisenberg Hamiltonian is invariant under spin rotation resulting in a degenerate ground state. The excitations are classified by the number of flipped spins in a ferromagnetic ground state. A so-called single-magnon excitation which has $S=1$ is created by a single spin flip. Although this thesis is not concerned with the ferromagnetic Heisenberg model, magnon excitations are also present in the fully spin-polarized phase of, e.g., the antiferromagnetic Heisenberg chain.

\section{$X X$ model 4.3}

The spin-1/2 $X X$ model [LSM61] is obtained from the Heisenberg Hamiltonian in Eq. (4.2) by setting the exchange coupling between the $z$ components of nearest-neighbor spins to zero. Using the Jordan-Wigner transformation [LSM61], the $X X$ model can be mapped to non-interacting spinless fermions and is hence exactly solvable. Its Hamiltonian is given by

$$
H_{X X}=J \sum_{j=1}^{L-1}\left(S_{j}^{x} S_{j+1}^{x}+S_{j}^{y} S_{j+1}^{y}\right) .
$$

For the $X X$ model different observable quantities such as the dynamical spin structure factor can be calculated exactly [DK98, DKS00]. This allows for a comparison to numerical results in Sec. 5.2.3. 


\section{\begin{tabular}{l|l} 
Superexchange interactions & 4.4
\end{tabular}}

The following brief review of superexchange interactions is mainly based on Refs. [Faz99, Blu01, Goo08] which may also be consulted for further reading. An aspect also reviewed here is the occurrence of ferromagnetic superexchange which is relevant for the study of the material $\mathrm{BaCu}_{2} \mathrm{~V}_{2} \mathrm{O}_{8}$ in Chapter 7 .

In the previous discussion of exchange interactions in Sec. 4.2, only the so-called direct exchange between adjacent sites in the single-orbital Hubbard model has been considered. In this case, the electrons may gain kinetic energy by virtual hopping between neighboring sites in the presence of a Coulomb repulsion. However, in many materials direct exchange does not represent the dominant mechanism for explaining their magnetic properties. The reason is simply that there is not sufficient overlap between the neighboring orbitals of the magnetic ions. This is the case for many antiferromagnetic insulators such as transition metal compounds.

Therefore, one oftentimes needs to consider longer-ranged so-called indirect exchange interactions. Such indirect exchange mechanisms are important in the description of many antiferromagnetic insulators. For instance, the 3D ionic solid in which the magnetic $\mathrm{Mn}^{2+}$ cations are separated with nonmagnetic intermediary $\mathrm{O}^{2-}$ anions on a simple cubic lattice. The antiferromagnetic order in this material is formed due to a dominating indirect exchange interaction mediated by the orbitals of the nonmagnetic ions in-between the magnetic moments. This is known as superexchange [Kra34, And50, And63]. In order to understand it, an even further idealized example adopted from Ref. [Faz99] is given in Fig. 4.2. The two cations, $\mathrm{A}$ and $\mathrm{B}$, each have one electron in the $d$ orbital. The intermediary anion is chosen as $\mathrm{O}^{2-}$ having a filled $p$ shell. The overlap between the cationic $d$ orbitals with the anionic $p$ orbital in-between allows the $p$ electrons to occupy these $d$ orbitals with a nonvanishing probability. This gives rise to an effective exchange interaction between the spatially separated $d$ orbitals of the cations.

Superexchange can be obtained by considering the energetics of several possible configurations in a perturbative treatment as done in Sec. 5.2 of Ref. [Faz99]. In this consideration, competing effects have to be considered. On the one hand, there is a kinetic exchange by virtual electron transfers between overlapping orbitals leading to double occupancies. Due to the Pauli exclusion principle antiferromagnetic exchange is energetically more favorable in this case since the indicated hopping in Fig. 4.2 is allowed. The magnitude of the kinetic exchange depends crucially on the overlap between the $d$ orbitals and the $p$ shell of the

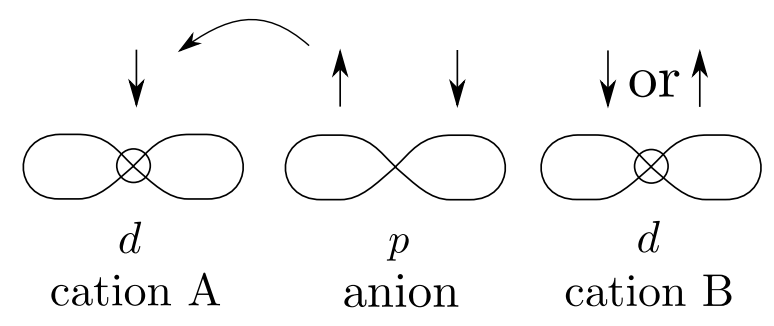

Figure 4.2: (Figure based on Fig. 5.1 in Ref. [Faz99]) Idealized example illustrating the superexchange between two magnetic cations $\mathrm{A}$ and $\mathrm{B}$ which is mediated by an intermediary anion. Here the cationic $d$ orbitals are singly-occupied and the $p$ shell of the anion is filled. This allows for virtual hopping processes. One of them is indicated by the arrow. 
intermediary anion. The overlap depends on the angle of the cation-anion-cation bond. An angle of about $180^{\circ}$, as depicted in Fig. 4.2, typically leads to an antiferromagnetic alignment of the spins.

On the other hand, the superexchange can also be ferromagnetic in certain cases. This is less common since the contribution leading to the antiferromagnetic exchange explained above is generally dominant. Ferromagnetic superexchange can occur when the virtual electron transfer through an intermediary anion is for instance from a half-filled to an empty $d$ orbital. Ferromagnetic ordering is also favored for orthogonal orbitals, but this contribution is usually small as the magnetic cations are spatially separated. In this case there is no virtual electron transfer and the corresponding exchange between the spins is a ferromagnetic potential exchange. For a more in-depth treatment of superexchange, a high number of excited orbital states has to be considered, see Ref. [ZP73].

In order to predict whether the net magnetic superexchange is antiferromagnetic or ferromagnetic, there exist the semi-empirical Goodenough-Kanamori rules. ${ }^{5}$ These have originally been formulated by Goodenough [Goo55, Goo58, Goo60] and have been further refined by Kanamori [Kan59]. Mainly based on the electron occupancies in atomic orbitals and symmetry relations, the semi-empirical rules allow for a quantitative prediction of the sign of the exchange interactions. They predict that a crossover from antiferromagnetic to ferromagnetic superexchange is possible at a bond angle of about $90^{\circ}$.

\section{Dzyaloshinskii-Moriya interactions $\mid 4.5$}

Note that parts of the present Section 4.5 have already been published in Ref. [THP $\left.{ }^{+} 16\right]$.

Since the spin-1/2 Heisenberg antiferromagnet has a ground state characterized by strong quantum fluctuations, it is therefore highly unstable even to small perturbations which may completely change the ground-state excitations of the antiferromagnetic Heisenberg model [OA97, Zvy12]. One example for such a perturbation are Dzyaloshinskii-Moriya (DM) interactions. First postulated by Dzyaloshinskii using a combination of phenomenology and symmetry considerations based on Landau theory [Dzy58], these interactions could be identified as a consequence of spin-orbit coupling by Moriya [Mor60a, Mor60b] who developed a general theory of anisotropic superexchange taking into account spinorbit coupling by a perturbative treatment. Here the spin-orbit interaction in the magnetic ions is modeled as $\sim \lambda \mathbf{L} \cdot \mathbf{S}$ with a small constant $\lambda \ll 1$. The DM interaction is an antisymmetric exchange interaction of the general form

$$
H_{\mathrm{DM}}=\mathbf{D}_{12} \cdot\left(\mathbf{S}_{1} \times \mathbf{S}_{2}\right)
$$

where $\mathbf{D}_{12}$ is a constant vector, the DM vector. The interaction is linear with respect to spin-orbit coupling $\left|\mathbf{D}_{12}\right| \sim \lambda$. When the crystal symmetry is sufficiently low, it is the largest contribution from anisotropic superexchange [Mor60b]. Moreover, small DM interactions in addition to symmetric exchange of the form $\mathbf{S}_{j} \cdot \mathbf{S}_{j+1}$ also explain a very small spontaneous magnetization known as "weak ferromagnetism" in antiferromagnetically ordered crystals [Dzy58, Mor60a]. The reason is that DM interactions lead to a slight misalignment of the spins resulting in a net ferromagnetic moment.

\footnotetext{
${ }^{5}$ They are sometimes also called Goodenough-Kanamori-Anderson rules.
} 
DM interactions are relevant in quasi-1D compounds with alternating crystal axes such as copper pyrimidine dinitrate $(\mathrm{Cu}-\mathrm{PM})\left[\mathrm{INN}^{+} 97, \mathrm{YIA}^{+} 01, \mathrm{FAG}^{+} 00\right]$ studied in Chapter 6. In $\mathrm{Cu}-\mathrm{PM}$, the alternating crystal axes give rise to staggered DM interactions with a factor of $(-1)^{j}$ [OA97] in the following chain Hamiltonian

$$
H_{\mathrm{DM}}=\sum_{j}(-1)^{j} \mathbf{D} \cdot\left(\mathbf{S}_{j} \times \mathbf{S}_{j+1}\right)
$$

Here only nearest-neighbor DM interactions along the chain are taken into account. Now the DM term in Eq. (4.10) is added as a perturbation to the antiferromagnetic Heisenberg Hamiltonian:

$$
H=\sum_{j}\left[J \mathbf{S}_{j} \cdot \mathbf{S}_{j+1}+h_{z} S_{j}^{z}+(-1)^{j} \mathbf{D} \cdot\left(\mathbf{S}_{j} \times \mathbf{S}_{j+1}\right)\right]
$$

Moreover, a uniform magnetic field $H$ is applied along the $z$ direction in Eq. (4.11) above, i.e., $H=h_{z}$. This contribution is included as the excitations will be studied in dependence of the magnetic field in this thesis. For simplicity, we first neglect the effects of the anisotropic $g$-tensor in materials such as $\mathrm{Cu}-\mathrm{PM}$. Later on, we shall see that it leads to a similar effect as the DM interactions.

\section{Effective model $\mid 4.5 .1$}

DM interactions can be eliminated [PC76, Mat77] by a staggered rotation of the spin operators about the direction of the DM vectors $\mathbf{D}_{j}=(-1)^{j} \mathbf{D}$ as long as $|\mathbf{D}| \ll J[\mathrm{OA} 97$, AO99]. The rotation angle is $(-1)^{j} \arctan (|\mathbf{D}| / J)$. For $|\mathbf{D}| \ll J$, the transformation can be linearized (see, e.g., Ref. [FO12]) and one obtains

$$
\mathbf{S}_{j} \rightarrow \mathbf{S}_{j}+(-1)^{j} \frac{\mathbf{D}}{2 J} \times \mathbf{S}_{j}
$$

This redefinition of the spin operators generates a transverse staggered magnetic field perpendicular to both the longitudinal applied field and D. Then the Hamiltonian in Eq. (4.11) takes the simpler form

$$
H=J \sum_{i} \mathbf{S}_{i} \cdot \mathbf{S}_{i+1}+h_{z} \sum_{i} S_{i}^{z}+h_{x} \sum_{i}(-1)^{i} S_{i}^{x}
$$

involving only a transverse staggered field $h_{\text {stag }}=h_{x}$ instead of the more complicated vector products. The staggered field is chosen along the $x$ direction and its strength is proportional to the uniform field, $h_{x}=c h_{z}$. The Hamiltonian in Eq. (4.13) is used for the MPS computations in Chapters 5 and 6.

The effect of the initially neglected anisotropy of the $g$ tensor in a more general Zeeman term $\left(\sum_{j} \mathbf{H} \cdot g \cdot \mathbf{S}_{\mathbf{j}}\right)$ also generates a staggered magnetic field whose transverse component can also be included in the material parameter $c$, whereas the longitudinal component we assume to be very small and therefore negligible, see for instance Ref. [FO12]. Note that the elimination of the DM interactions also leads to a very small exchange anisotropy in the Heisenberg model [Ess99] which is ignored in the following. 


\section{Quantum sine-Gordon model $\mid 4.5 .2$}

In some cases, it will be useful to compare the numerical MPS calculations for the effective Hamiltonian in Eq. (4.13) to previous field theoretical results. This will be discussed in Chapter 6 . To this end, a short account of the quantum sine-Gordon model is given in the following.

For $h_{\text {stag }} \ll J$, the low-energy behavior of the model in Eq. (4.13) above can be treated by Abelian bosonization and is given by the quantum sine-Gordon model with Lagrangian density [OA97, ET98, AO99, Ess99],

$$
\mathcal{L}=\frac{1}{2}\left(\partial_{\mu} \Phi\right)^{2}+\lambda\left(h_{\text {stag }}\right) \cos \left(\beta\left(h_{z}\right) \Theta\right)
$$

Here $\Phi$ is a boson field and $\Theta$ the corresponding dual field and the coefficient $\lambda\left(h_{\text {stag }}\right)$ is field dependent. Another field dependence is included in the coupling $\beta\left(h_{z}\right)$ which is calculated from the exact solution of the Heisenberg model in only a uniform magnetic field $\left(h_{\text {stag }}=0\right)$. The coupling $\beta\left(h_{z}\right)$ should not be confused with the inverse temperature $\beta$ carrying no argument. This approximation is assumed to be justified for $h_{\text {stag }} \ll h_{z}$. The model in Eq. (4.14) is exactly solvable [Lut76, ZZ79, BT79, Kor79] and the lowenergy elementary excitations are known to be solitons and antisolitons which interact and propagate as robust localized quasiparticles with mass $M_{S}$ and charge $Q= \pm 1$. The soliton mass was determined for magnetic fields $h_{z}$ comparable to $J\left(h_{\text {stag }} \ll J\right)$ [EFH03]:

$$
\frac{M_{S}}{J}=\frac{2 v}{\sqrt{\pi}} \frac{\Gamma\left(\frac{\xi}{2}\right)}{\Gamma\left(\frac{1+\xi}{2}\right)}\left[\frac{\Gamma\left(\frac{1}{1+\xi}\right)}{\Gamma\left(\frac{\xi}{1+\xi}\right)} \frac{g \mu_{\mathrm{B}} \pi A_{x}}{2 J v} h_{\mathrm{stag}}\right]^{(1+\xi) / 2} .
$$

Here $v$ is the dimensionless spin velocity. Although Equation (4.15) is exact [Zam95], the field-dependent amplitude $A_{x}$ for the bosonized expression of a spin operator is not known analytically. Therefore, both $v$ and $A_{x}$ were determined via DMRG for static correlation functions [EFH03]. The parameter $\xi$ is related to the field-dependent coupling $\beta$ via $\xi=\beta\left(h_{z}\right)^{2} /\left(8 \pi-\beta\left(h_{z}\right)^{2}\right)$. It is important to stress that the soliton and antisoliton are found at incommensurate wave vectors $q_{s}= \pm q_{0}$ and $q_{s}=\pi \pm q_{0}$ as sketched in Fig. 4.3. The shift $q_{0}=2 \pi m\left(h_{z}\right)$ is given in terms of the total magnetization per site $m\left(h_{z}\right)$ [AO99]. In order to predict the single-soliton resonance at $q=0$ resp. $q=\pi$, the field theory assumes a Lorentz invariant dispersion

$$
E_{S}=\sqrt{M_{S}^{2}+h_{z}^{2}}
$$

which is sketched as a gray solid line in Fig. 4.3. Further elementary excitations, the breathers, consist of soliton-antisoliton bound states. The mass gap of the $n$th breather depends both on the soliton mass $M_{S}$ and on $\xi$ as

$$
M_{n}=2 M_{S} \sin \left(\frac{n \pi \xi}{2}\right) \text {. }
$$

The number of breathers is restricted, i.e., $n=1,2, \ldots,\left\lfloor\xi^{-1}\right\rfloor$. Breather excitations do not carry any soliton charge $Q=0$. 


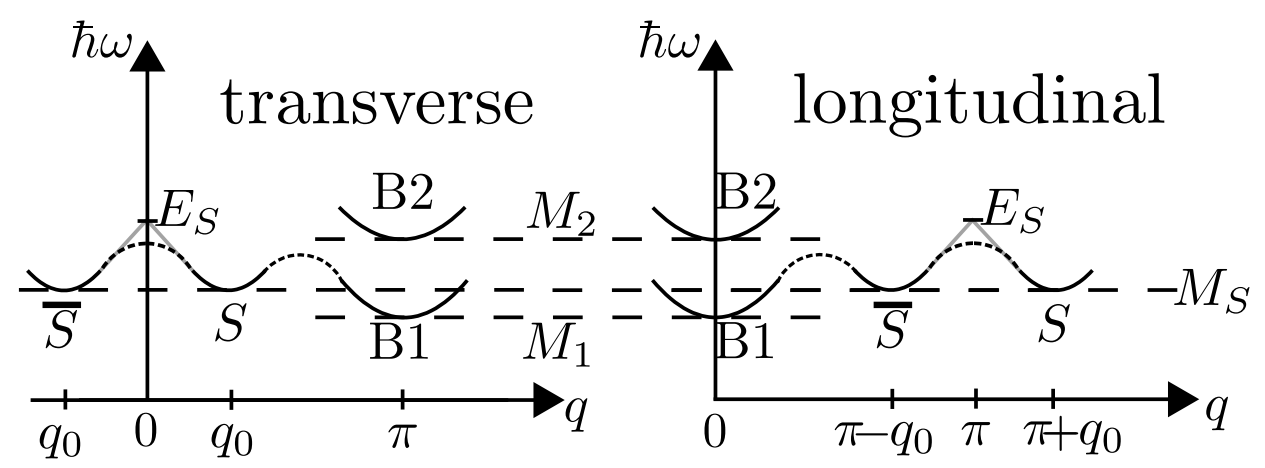

Figure 4.3: (Published in Ref. $\left[\mathrm{THP}^{+} 16\right]$.) Schematic sketch of the low-energy modes of the transverse (left panel) and longitudinal (right panel) dynamical spin structure factor. Here B1 and B2 label the first two breathers, whereas $S(\bar{S})$ denotes the (anti)soliton. As discussed in Chapter 6 , note that the modes are actually connected to each other via the dashed lines, while the field theory (thin gray lines) assumes a linear dispersion of the soliton around $q=0$.

\section{Alternating Heisenberg chain 4.6}

Another model studied in this thesis is the spin- $1 / 2$ alternating Heisenberg chain which is a further generalization of the uniform Heisenberg antiferromagnet in Eq. (4.2). Its Hamiltonian is given by

$$
H=\sum_{j}\left[J_{\text {intra }} \mathbf{S}_{j, 1} \cdot \mathbf{S}_{j, 2}+J_{\text {inter }} \mathbf{S}_{j, 2} \cdot \mathbf{S}_{j+1,1}\right] .
$$

Here strongly alternating systems in one dimension are studied. In this case, the condition $J_{\text {intra }} \gg J_{\text {inter }}$ holds and one calls such systems dimerized. Assuming antiferromagnetic exchange $\left(J_{\text {inter }}, J_{\text {intra }}>0\right)$, this terminology becomes clear since the dominant interaction $J_{\text {intra }}$ couples two neighboring spins into so-called dimers. Thus, $J_{\text {intra }}$ is referred to as intradimer coupling and describes the interaction of the two spins $\mathbf{S}_{j, 1}$ and $\mathbf{S}_{j, 2}$ constituting the $j$ th dimer in Eq. (4.18). The ground-state of an isolated dimer is a spin singlet

$$
\left|E_{0, \text { dimer }}\right\rangle=\frac{1}{\sqrt{2}}(|\uparrow \downarrow\rangle-|\downarrow \uparrow\rangle)
$$

with energy $E_{0, \text { dimer }}=-3 J_{\text {intra }} / 4$. The excited states of a single dimer are triplet states

$$
\begin{aligned}
\left|E_{1, \text { dimer }}\right\rangle & =\frac{1}{\sqrt{2}}(|\uparrow \downarrow\rangle+|\downarrow \uparrow\rangle), \\
\left|E_{2, \text { dimer }}\right\rangle & =|\uparrow \uparrow\rangle, \\
\left|E_{3, \text { dimer }}\right\rangle & =|\downarrow \downarrow\rangle
\end{aligned}
$$

with energy $E_{\text {triplet }}=J_{\text {intra }} / 4$ carrying a spin of $S=1$. Such excitations in the chain of coupled dimers are henceforth referred to as triplons [SU03]. ${ }^{6}$ The ground state of a strongly dimerized chain is a product state of dimer singlets in which a small amount of two-triplon fluctuations with a total spin of zero is admixed due to the interdimer coupling

\footnotetext{
${ }^{6}$ In order to avoid confusion about the terminology, note that these excitations are also called magnons in other works such as Ref. [TLJ $\left.{ }^{+} 12\right]$.
} 
$J_{\text {inter }}\left[\mathrm{TLJ}^{+}{ }^{+} 2\right]$. The interdimer exchange interactions make the single-triplon excitations mobile. They are allowed to hop between adjacent dimers along the chain, while they obey a hard-core constraint. The constraint signifies that a single site cannot be occupied by two triplons simultaneously. Their dispersion can be expanded up to lowest order in the perturbation $\alpha=J_{\text {inter }} / J_{\text {intra }}$ is given by

$$
\frac{\omega(q)}{J_{\text {intra }}}=1-\frac{J_{\text {inter }}}{J_{\text {intra }}} \cos (a q)
$$

where $a$ is the lattice periodicity [Har73]. ${ }^{7}$ The condition $J_{\text {intra }} \gg J_{\text {inter }}$ guarantees a large gap compared to the bandwidth of the single-triplon dispersion. The dispersion up to fifth order in $\alpha=J_{\text {inter }} / J_{\text {intra }}$ is given by

$$
\frac{\omega(q)}{J_{\text {intra }}}=\sum_{l=0}^{5} a_{l} \cos (l a q) .
$$

This result is obtained in Ref. [BRT99] where the coefficients $a_{l}$ are calculated as polynomials in $\alpha$ :

$$
\begin{array}{llllll}
a_{0}= & 1 & -\frac{1}{16} \alpha^{2} & +\frac{3}{64} \alpha^{3} & +\frac{23}{1024} \alpha^{4} & -\frac{3}{256} \alpha^{5}, \\
a_{1}= & -\frac{1}{2} \alpha & -\frac{1}{4} \alpha^{2} & +\frac{1}{32} \alpha^{3} & +\frac{5}{256} \alpha^{4} & -\frac{35}{2048} \alpha^{5}, \\
a_{2}= & -\frac{1}{16} \alpha^{2} & -\frac{1}{32} \alpha^{3} & -\frac{15}{512} \alpha^{4} & -\frac{283}{18432} \alpha^{5}, \\
a_{3}= & & -\frac{1}{64} \alpha^{3} & -\frac{1}{48} \alpha^{4} & -\frac{9}{1024} \alpha^{5}, \\
a_{4}= & & & -\frac{5}{1024} \alpha^{4} & -\frac{67}{9216} \alpha^{5}, \\
a_{5}= & & & & -\frac{7}{4096} \alpha^{5} .
\end{array}
$$

The expression for the dispersion in Eqs. (4.24) and (4.25) will be used in Chapter 7.

Further ground-state excitations of the dimerized chain are studied in [BRT99, KU00]. For instance, two-triplon excitations lead to continua at higher frequencies. For $J_{\text {intra }} \gg J_{\text {inter }}$, they are well separated from the single-triplon dispersion at lower frequency. There is a two-triplon bound state with total spin $S=1$ just below the continua [BRT99].

Last but not least, there are many compounds which can be effectively described by this model. See Ref. [BRT99] for a list. In dimerized materials different inequivalent exchange paths are linked in such a way that they can be described by alternating exchange couplings $J_{\text {inter }}-J_{\text {intra }}-J_{\text {inter }}-J_{\text {intra }}-\ldots-J_{\text {inter }}-J_{\text {intra }}$. One of these realizations is the quasi$1 \mathrm{D}$ compound copper nitrate [Mor70, $\left.\mathrm{TBR}^{+} 03\right]$ in which both exchange interactions are antiferromagnetic and their ratio is $J_{\text {inter }} / J_{\text {intra }} \approx 0.227$, where $J_{\text {intra }}=0.443 \pm 0.002 \mathrm{meV}$ $\left[\mathrm{TLJ}^{+} 12\right]$. The model in Eq. (4.18) will be used in the study of another dimer-chain compound, namely $\mathrm{BaCu}_{2} \mathrm{~V}_{2} \mathrm{O}_{8}$ [VMB90, HKI04], in Chapter 7.

\footnotetext{
${ }^{7}$ Due to the two alternating exchange couplings, one has a lattice periodicity of $a=2$ lattice spacings.
} 



\section{Proof-of-principle MPS frequency-space calculations at $T>0 \quad 5$}

The main content of this chapter is published as a Rapid Communication in Physical Review B [TMPH14] and therefore some parts of the following chapter are directly adopted from the manuscript of Ref. [TMPH14]. In particular, this is the case for Secs. 5.2.3 and 5.3.2. Moreover, Section 5.4 represents the supplemental material of Ref. [TMPH14]. The manuscript as well as the supplemental material were written mainly by me and revised together with my coauthors Salvatore R. Manmana, Andreas Honecker, and Thomas Pruschke. The MPS calculations have been performed by me and I implemented the related MPS code from scratch.

Furthermore, it is pointed out that the resolution of the zero-temperature Chebyshev expansions is specified incorrectly in Ref. [TMPH14]. Thus, the resolution is corrected in Figs. 5.7(a), 5.7(b), and 5.9 in the present chapter.

\section{\begin{tabular}{l|l} 
Introduction & 5.1
\end{tabular}}

The main scope of this chapter is to present proof-of-principle results obtained by a newly developed approach for the calculation of finite-temperature spectral functions of 1D strongly correlated quantum systems. As explained in Sec. 3.6, the method combines the purification of the finite-temperature density operator with a moment expansion of the dynamical correlation function and operates in the framework of MPS.

The flexibility of the approach is shown by implementing both a Chebyshev expansion of the spectral function and, alternatively, a continued fraction expansion (CFE). The potential of the method is demonstrated by proof-of-principle calculations for the $X X$ model introduced in Eq. (4.8). The accuracy of zero-temperature MPS methods crucially depends on the entanglement scaling which is closely related to the eigenvalue spectra of reduced density matrices corresponding to a bipartition of the system. Therefore, such spectra are studied in dependence of the Chebyshev iteration in order to assess the applicability of frequency-domain methods at finite temperature. Then the finite-temperature properties of dynamical spectral functions of isotropic spin- $1 / 2$ Heisenberg chains with symmetry breaking Dzyaloshinskii-Moriya interactions are studied in magnetic fields.

The second purpose of this chapter is the calculation of static thermodynamic observables in the course of an imaginary-time evolution of an MPS purification, as explained in Sec. 3.6.2. These data are compared to existing exact results allowing to check the accuracy of imaginary-time evolution algorithms. This is crucial as the methods are used to compute the thermal states $\left|\Psi_{T}\right\rangle$ which constitute the starting point in the calculation of a spectral function at temperature $T$. 


\section{Results for the $X X$ model 5.2}

In order to assess the accuracy of the MPS frequency-space approach at finite temperatures, the antiferromagnetic $X X$ model in zero magnetic field introduced in Sec. 4.3 represents a good test case. By means of the Jordan-Wigner transform [LSM61], this system can be mapped to free fermions and is hence exactly solvable.

\section{\begin{tabular}{l|l} 
Thermodynamics & 5.2 .1
\end{tabular}}

First of all, the accuracy of the MPS-based imaginary-time evolution used to obtain the thermal state $\left|\Psi_{T}\right\rangle$ at temperature $T$ is checked. To this end, the temperature dependence of the specific heat

$$
C(T)=\left(\frac{\partial U}{\partial T}\right)_{V, N}=\left[\left\langle H^{2}\right\rangle_{T}-\langle H\rangle_{T}^{2}\right] / T^{2}
$$

is calculated. The MPS results for this static thermodynamic quantity are calculated using the purification trick (see Eq. (3.111)) and the TDVP algorithm for imaginary-time evolution. The time step is chosen as $\Delta t=0.01{ }^{1}$ These are shown for a finite chain of $L=50$ sites and an internal MPS bond dimension of $m=200$ in Fig. 5.1(a) where they are directly compared to the exact result. There is excellent agreement for $C(T)$, which is also reflected by the absolute deviation from the exact results $\left|C_{\text {exact }}(T)-C_{\text {TDVP }}(T)\right| / L$ plotted as a function of the inverse temperature $\beta=\left(k_{\mathrm{B}} T\right)^{-1}$ in Fig. 5.1(b) for different values of $m$. Note that $k_{\mathrm{B}}=1$ is adopted for the calculations. In particular, the choice of $m=200$ ensures that the absolute deviation of $C(T)$ is only of the order of $10^{-9}$ for $\beta \leq 10$. Therefore, it is justified to assume that the error accumulated in the course of the imaginary-time evolution only represents a negligible source of errors with respect to the subsequent calculation of spectral functions in the following section.

Moreover, Figure 5.1(b) also contains the absolute deviation between the exact results and the MPS-based Lanczos time evolution using a time step of $\Delta t=0.005$. Moreover, the parameters specified in Sec. 3.4.3 were used. For $m=200$, the deviation is very small $\left(<10^{-11}\right)$. The Lanczos time-evolution method has been employed for the imaginarytime evolution in the first publication related to this thesis [TMPH14] since the TDVP algorithm has not yet been introduced at that time.

\section{\begin{tabular}{l|l} 
Reduced density-matrix spectra & 5.2 .2
\end{tabular}}

The present section is a prelude to the calculation of the dynamical spin structure factor in the following subsection. In the following paragraphs, the applicability of frequencydomain methods at finite temperatures is considered. The accuracy of MPS methods is closely related to the entanglement properties of the underlying system (see Sec. 3.3.1). Hence it is useful to study the entanglement generated in the course of the Chebyshev

\footnotetext{
${ }^{1}$ The imaginary-time evolution is not the bottleneck in the numerics since the frequency-domain calculations are computationally more costly. Thus, the time step is not optimized for utmost efficiency here.
} 

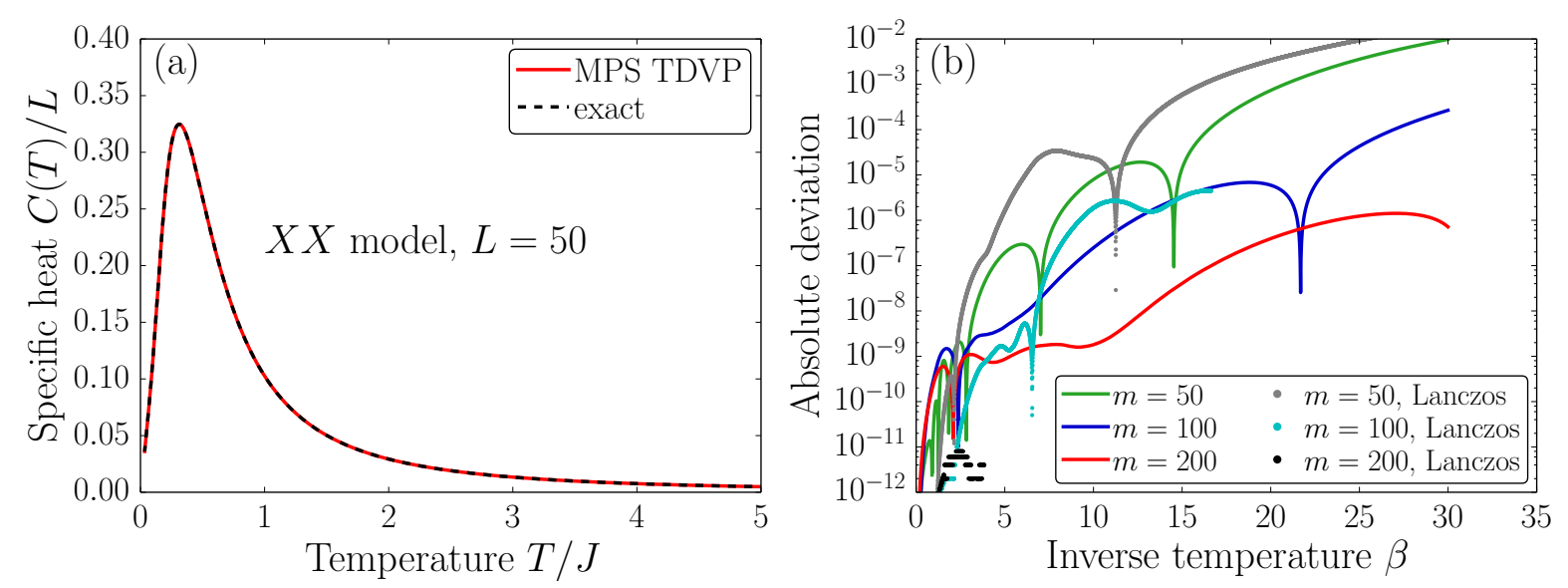

Figure 5.1: Results for the $X X$ model: (a) Specific heat $C(T)$ as a function of temperature calculated using the MPS-based TDVP algorithm for imaginary-time evolution compared to exact results for a chain of length $L=50$ and an internal MPS bond dimension of $m=200$. The time step is $\Delta t=0.01$ for one full sweep. (b) Absolute deviation $\left|C_{\text {exact }}(T)-C_{\text {TDVP }}(T)\right| / L$ between the exact and TDVP results (solid lines) for a few selected values of $m$. Moreover, the absolute deviation $\left|C_{\text {exact }}(T)-C_{\text {Lanczos }}(T)\right| / L$ is also plotted for MPS-based Lanczos time evolution (solid circles) using $\Delta t=0.01$ for a full sweep.

recursion at $T>0$. Since there is no commonly accepted measure for the characterization of entanglement at $T>0$ [AFOV08, ACL12], it can be more instructive to consider the reduced density matrix eigenvalue spectra and, in particular, their decay. This information helps to understand the generation of entanglement by frequency-domain methods at finite temperature. Therefore, the reduced density-matrix eigenvalue spectra are plotted in dependence of the Chebyshev iteration $n$ in Fig. 5.2. Each panel of this figure contains the spectra for a given temperature and $n=0$ denotes the starting vector. The results are obtained for an $X X$ chain with $L=50$ physical sites. The system is partitioned into two parts by a cut through the center of the chain.

At high temperature, it is a striking feature that $n=0$ spectrum falls off a lot faster in Fig. 5.2(a) than at lower temperature in Figs. 5.2(b)-(e). This can be understood by the fact that the entanglement of the starting vector is built up during the previous imaginary-time evolution lowering the temperature. In all panels of Fig. 5.2, the decay of the eigenvalue spectra becomes slower with increasing Chebyshev iterations.

Figure 5.2(f) contains zero-temperature results for the reduced density matrix eigenvalue spectra obtained without the purification. ${ }^{2}$ The zero-temperature spectra have been studied when the Chebyshev MPS method was introduced $\left[\mathrm{HWM}^{+} 11\right]$. In particular, it was found that the decay of the spectra saturates for higher iterations at $T=0$. It is now interesting that, in the present work, the saturation of the spectra is also observed at finite temperature in Fig. 5.2(a)-(e).

The decay of the eigenvalue spectra is crucial for the accuracy of DMRG methods as the eigenvalues represent the weights of the corresponding states. For the zero-temperature Chebyshev MPS method it was found that including all states above a threshold of about $10^{-3}$ gives accurate results $\left[\mathrm{HWM}^{+} 11\right]$. This threshold is a few orders of magnitude higher than in ground-state DMRG calculations where truncation errors are typically

\footnotetext{
${ }^{2}$ Note that the Hamiltonian was rescaled with the many-body bandwidth of the Liouvillian to allow for better comparability.
} 


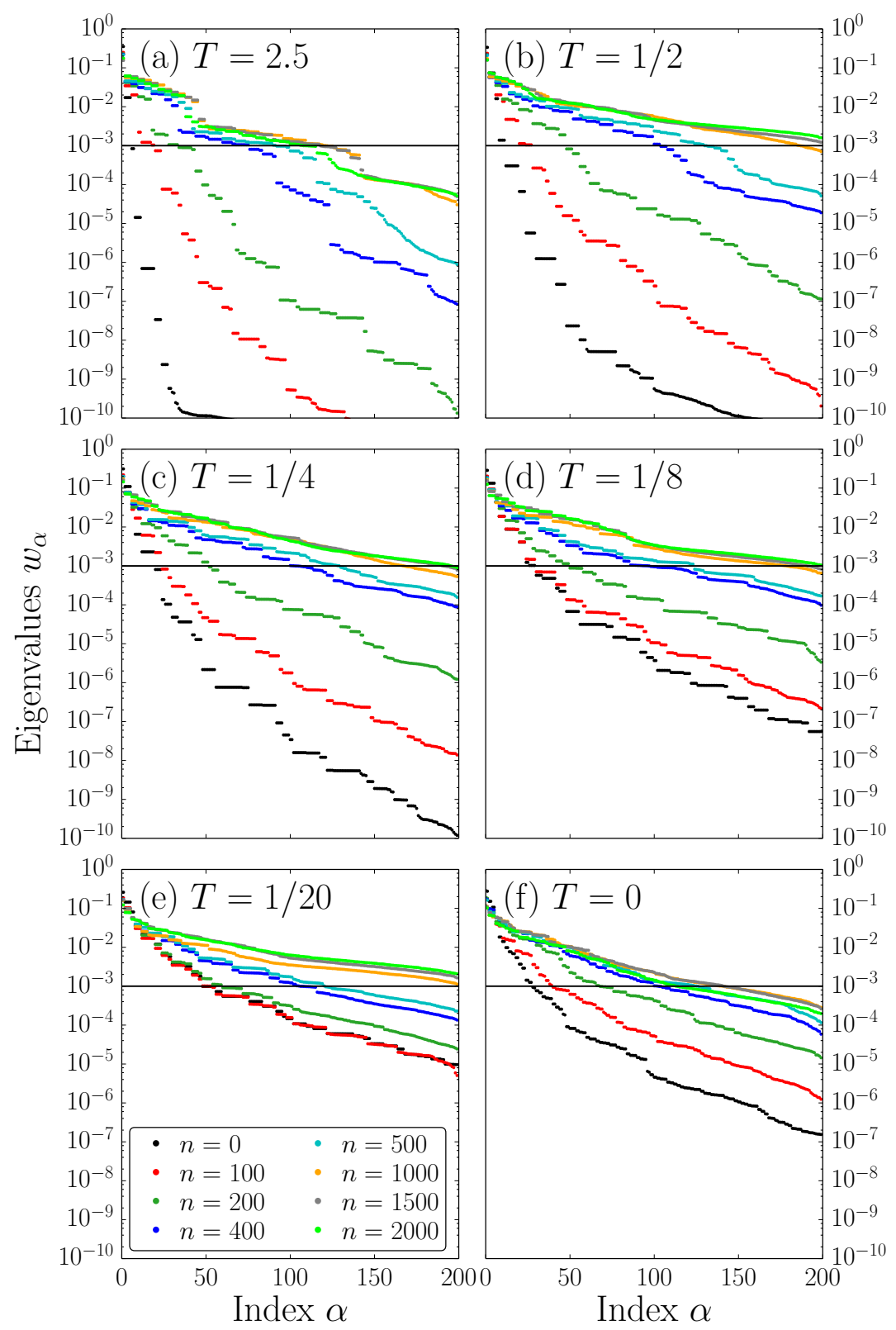

Figure 5.2: Results for an $X X$ chain with $L=50$ physical sites: Evolution of the reduced density-matrix eigenvalue spectra with the number of Chebyshev iterations $n$ for a bipartition through the center of the chain. Each panel shows the eigenvalues $w_{\alpha}=\left|s_{\alpha}\right|^{2}$ of the reduced density matrices for a fixed temperature. Panels (a)-(e) show results obtained with a purified wave function at $T>0$. Panel (f) contains zero-temperature data obtained without the purification. The solid horizontal lines at $10^{-3}$ are added for a better comparison.

between $10^{-6}$ and $10^{-8}$. In the nontrivial test case of the $X X$ model treated here, the finite-temperature eigenvalue spectra decay also to about $10^{-3}$ if one keeps $m=200$ eigenstates. This is roughly the same order of magnitude as for the $T=0$ data in Figure $5.2(\mathrm{f})$. In fact, retaining only $m=100$ states causes no significant deviations in the spectral function as it can be seen in Fig. 5.4 further below.

As additional information, the error $\epsilon_{\text {compr }}$ made by the variational compression after each Chebyshev iteration is plotted in Fig. 5.3. It offers another source of guidance for 


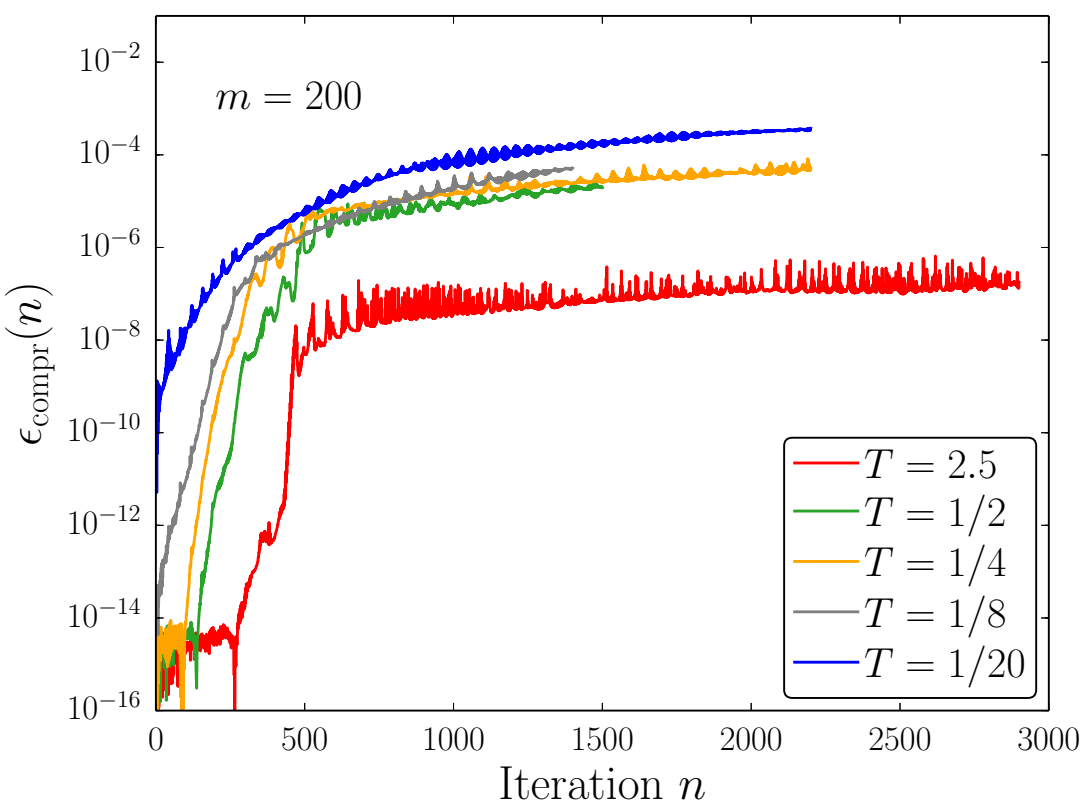

Figure 5.3: Results for an $X X$ chain with $L=50$ physical sites: Error of the variational compression $\epsilon_{\text {compr }}$ depicted in dependence of the Chebyshev iteration $n$ for a fixed internal MPS bond dimension of $m=200$ and a few selected temperatures.

the accuracy of the results - here specifically for the dynamical spin structure factors presented in the following section.

\section{Dynamical spin structure factor $\mid 5.2 .3$}

As a first proof-of-principle calculation for the Liouville-space formulation of MPS frequencydomain methods at $T>0$, the longitudinal spin structure factor $S_{z z}(k, \omega)$ of the $X X$ model (4.8) is computed in zero field. Since open boundary conditions are adopted, the spin operators in $k$ space are defined via particle-in-a-box eigenstates rather than plain waves

$$
S_{k}^{\alpha}=\sqrt{\frac{2}{L+1}} \sum_{j=1}^{L} \sin (k j) S_{j}^{\alpha}
$$

with respect to the quasi momenta $k=\pi n /(L+1)$ and integers $n=1, \ldots, L$ [BGJ04]. For the computation of $S_{z z}(k, \omega)$, the operator of interest then reads $A=\left(S_{k}^{z}\right)_{P} \otimes I_{Q}$.

A comparison to exact results is possible since the time-dependent spin correlation functions $\left\langle S_{i}^{z}(t) S_{j}^{z}(0)\right\rangle$ can be evaluated exactly [DK98, DKS00] and transformed into $\omega$ and $k$ space using Eq. (5.2) by

$$
S_{z z}(k, \omega)=\sum_{i, j} \frac{\sin (k i) \sin (k j)}{\pi(L+1)} \int \mathrm{d} t \mathrm{e}^{i \omega t-\eta^{2} t^{2} / 2}\left\langle S_{i}^{z}(t) S_{j}^{z}(0)\right\rangle,
$$


where $\left\langle S_{i}^{z}(-t) S_{j}^{z}(0)\right\rangle=\left\langle S_{i}^{z}(t) S_{j}^{z}(0)\right\rangle^{*}$ holds for negative times. ${ }^{3}$ The expectation value denotes the canonical thermodynamic average. Here $\eta$ introduces uniform Gaussian broadening needed for a comparison to Chebyshev expansions with Jackson damping. ${ }^{4}$

The MPS results are obtained by using the Liouvillian formulation of frequency-space dynamics in combination with Chebyshev expansions of the spectral function (order $N=1700)$. They are compared to exact results in Fig. 5.4 for a system of size $L=50$. Figure 5.4(a) shows the results for the longitudinal spin structure factor $S_{z z}(k=25 \pi / 51, \omega)$ at $T=\infty$ for different numbers of the internal MPS bond dimension $m$. Although the Gaussian broadening $\eta$ of the expansions is not strictly uniform by construction, the agreement with the exact result $(\eta=0.06)$ is excellent for $m=100$.

In Fig. 5.4(b) the temperature dependence of $S_{z z}(k=25 \pi / 51, \omega)$ is depicted for $m=100$. Since these MPS results are obtained with the same resolution, it is possible to study temperature effects. In this case, the thermal broadening of a van Hove singularity in one dimension is observed. It is resolved as the main feature of the dynamical spin structure factor in Fig. 5.4. The MPS results fit the exact curves well down to temperatures as low as $T / J=0.125$.

Moreover, for an infinite system the support of the $z z$ dynamical spin structure factor at $T=0$ and $k=25 \pi / 51$ also has a lower boundary at $\omega>0$ at which a finite jump in intensity occurs [DV06]. Above this gap the two-spinon continuum extends. The MPS results for finite temperatures shown in Fig. 5.4 suggest that the threshold is smeared out already at low temperature and confirm its disappearance at high temperature.

\footnotetext{
${ }^{3}$ There is also an exact expression for the dynamical spin structure factor in the thermodynamic limit, see Eq. (3.1) in Ref. [DVKB06].

${ }^{4}$ Lorentzian broadening, e.g., needed for a comparison to CFE results, is obtained for $\mathrm{e}^{i \omega t-\eta t}$ in the integral expression in Eq. (5.3).
} 


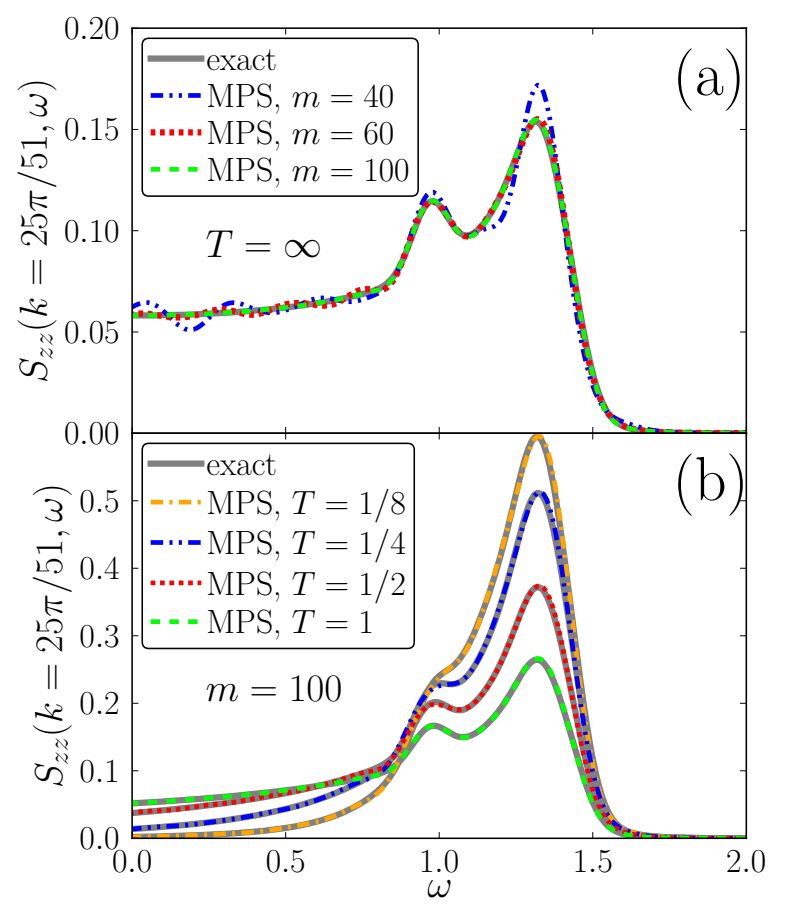

Figure 5.4: (Published in Ref. [TMPH14].) Finite-temperature DMRG calculations for the dynamical spin structure factor $S_{z z}(k=25 \pi / 51, \omega)$ of an open $X X$ chain with $L=50$ sites compared to exact results with Gaussian broadening $\eta=0.06(N=1700)$. Here $J=1$ is adopted. (a) At $T=\infty$, the accuracy is studied for different numbers for the MPS bond dimension $m$. (b) Temperature dependence of $S_{z z}(k=25 \pi / 51, \omega)$ compared to the exact solutions (solid lines) for $T=0.125,0.25,0.5$, and 1 (from top to bottom). 


\section{Results for the isotropic Heisenberg chain 5.3}

In the following two subsections, results for the isotropic Heisenberg chain are shown. Section 5.3.1 is concerned with static thermodynamic properties. The temperature dependence of the dynamical spin structure factor in the presence and absence of DzyaloshinskiiMoriya (DM) interactions is studied in a uniform magnetic field in Sec. 5.3.2. The DM interactions are incorporated into the model as a transverse staggered field (see Sec. 4.5). The dynamical spin structure factor is calculated by MPS-based Chebyshev expansions directly in the frequency domain. At $T>0$, the Liouville-space formulation is exploited providing further proof-of-principle results for the newly developed approach.

\section{\begin{tabular}{l|l} 
Thermodynamics in zero magnetic field & 5.3 .1
\end{tabular}}

For the isotropic spin-1/2 Heisenberg Hamiltonian in Eq. $(4.2)\left(J_{z}=J\right)$, there also exist exact results for the specific heat and the static magnetic susceptibility obtained via the thermodynamic Bethe ansatz in the limit $L \rightarrow \infty$ [EAT94, Klü98, KJ00, $\mathrm{WRC}^{+}$03]. The exact specific heat data is compared to those obtained for finite systems by imaginarytime evolution in Fig. 5.5(a). There is very good agreement between the exact and the MPS results down to low temperatures. The inset in Fig. 5.5(a) focuses on intermediate temperatures where the deviations from the infinite-system Bethe ansatz data are still minor but most pronounced. However, it is very likely that these appear due to the finite number of physical sites and the choice of OBCs. Indeed, the inset shows that the finitesize curves move closer towards the exact result for $L=\infty$ with increasing system size. Moreover, Fig. 5.5(a) also contains not exclusively TDVP data but also Lanczos timeevolution results for $L=40$ also underlining the similar reliability of this approach. ${ }^{5}$ A similar comparison between exact and MPS results for the static magnetic susceptibility is shown in Fig. 5.5(b). This thermodynamic observable is defined as

$$
\chi(T, h=0)=\left.\frac{\partial M}{\partial h}\right|_{h=0}=\left[\left\langle S_{z}^{2}\right\rangle_{T}-\left\langle S_{z}\right\rangle_{T}^{2}\right] / T .
$$

For $\chi(T)$ excellent agreement is found from intermediate to high temperatures. Due to finite-size and boundary effects there are increasing deviations from the exact results for an infinite system towards low temperature. These small discrepancies (at most 10\%) are more pronounced than in Fig. 5.5(a) showing the specific heat. The inset of Fig. 5.5(b) is focused on this region and reveals that the MPS curves for larger systems are found to approach the exact result $(L=\infty)$.

In order to control the error of the TDVP approach quantitatively, the calculations are performed for different internal bond dimensions of the MPS, i.e, $m=100,200$, and in some cases even $m=300$. Then the absolute difference between the $m=100$ and $m=200[m=200$ and $m=300]$ data is plotted for both $C(T) / L$ and $\chi(T) / L$ as a function of the inverse temperature $\beta$ in Fig. 5.6. The quantity serves as an error estimate and is below $10^{-5}$ for $\beta \leq 10$ if the difference between the $m=100$ and $m=200$ results is considered. Therefore, even a $m=100$ calculation gives accurate results for

\footnotetext{
${ }^{5}$ In fact, the Lanczos method was used to obtain the thermal states in Ref. [TMPH14] in which parts of this chapter have been published.
} 

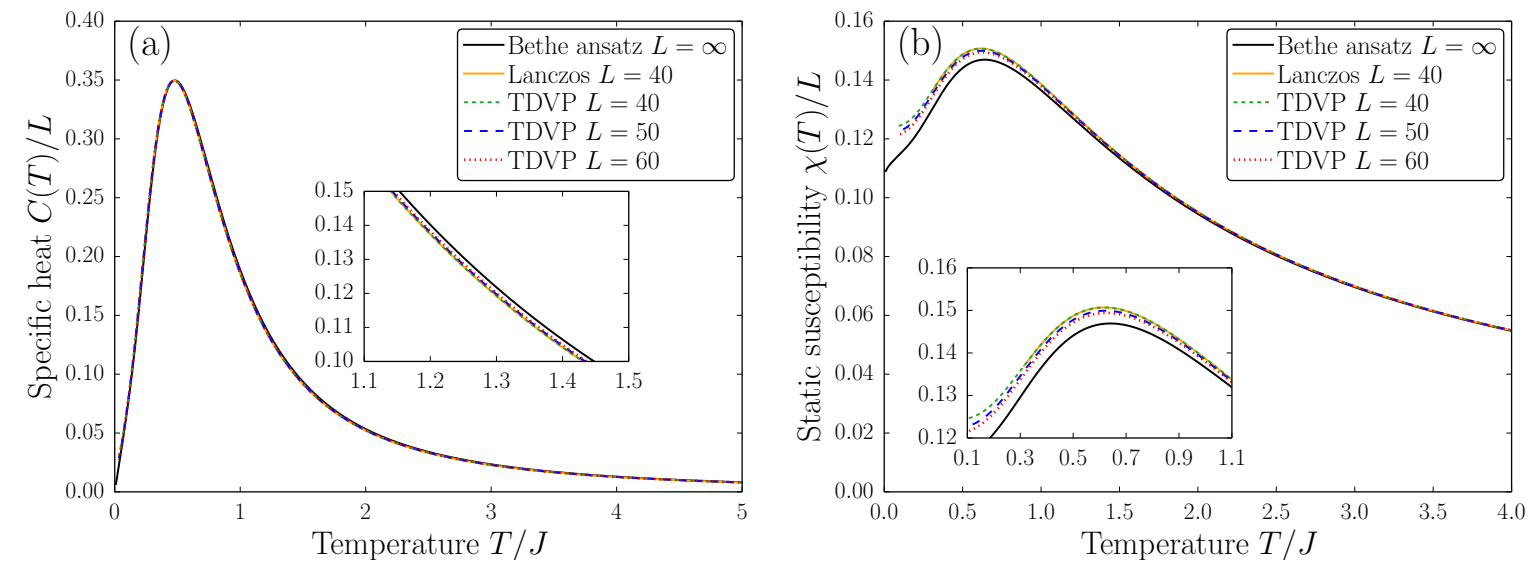

Figure 5.5: Results for the isotropic spin-1/2 Heisenberg chain in zero magnetic field: (a) Temperature dependence of the specific heat $C(T)$ calculated for finite systems with OBCs using the TDVP algorithm for imaginary-time evolution in comparison to exact Bethe ansatz results in the thermodynamic limit (taken from Ref. [Klü98]). For the smaller system of size $L=40$, there are also Lanczos time evolution data $(m=100)$. (b) Temperature dependence of the static magnetic susceptibility $\chi(T)$. In each panel, the inset zooms in on the results at intermediate (a) respectively low (b) temperature showing that the finite-system curves move closer towards the exact results with increasing system size. For the TDVP computations an internal MPS bond dimension of $m=200$ and the same time step as in Sec. 5.2.1 were used $(\Delta t=0.01)$.

this temperature range. The comparison between $m=200$ and $m=300$ shows that the improvements for $m=300$ are not very significant. Thus, $m=200$ or $m=250$ is sufficient to obtain highly accurate results. Moreover, it is also interesting that the normalized error estimates (e.g. for $m=100$ vs. $m=200$ ) as depicted in Fig. 5.6 behave in a very similar way, irrespective of the actual system size and over a broad temperature range.
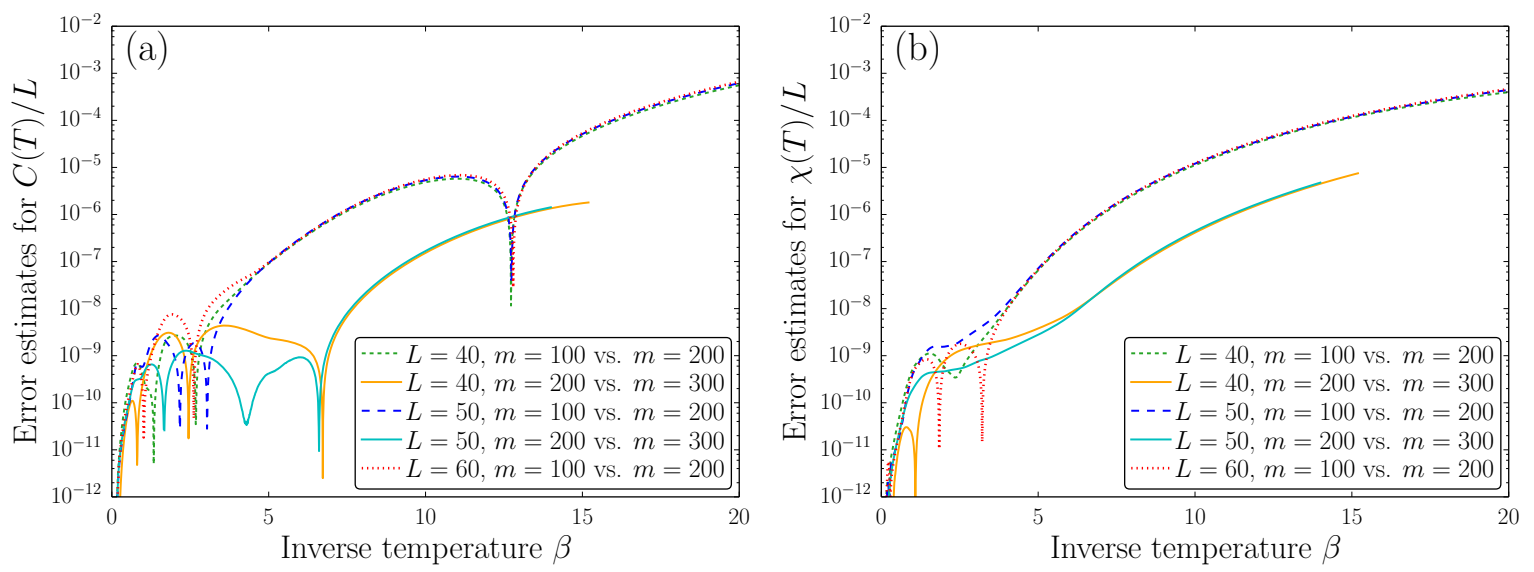

Figure 5.6: Results for the isotropic spin- $1 / 2$ Heisenberg chain: Temperature dependence of the absolute difference between the TDVP results for both (a) $C(T) / L$ and (b) $\chi(T) / L$ computed for different MPS bond dimensions $m=100$ and $m=200[m=200$ and $m=300]$ and system sizes. The quantity serves as an error estimate. 


\section{\begin{tabular}{l|l} 
Dynamical spin structure factor & 5.3 .2
\end{tabular}}

The present section features further proof-of-principle results for finite-temperature spectral functions obtained by MPS-based Chebyshev expansions directly in the frequency domain. The following study is motivated by quasi-1D compounds such as Cu-PM $\left[\mathrm{ZKKF} 04, \mathrm{ZCO}^{+} 11\right]$ and copper benzoate $\left[\mathrm{DDacR}^{+} 96, \mathrm{ANI}^{+}\right.$03]. These materials possess alternating crystal axes giving rise to nearest-neighbor Dzyaloshinskii-Moriya (DM) interactions and an alternating $g$ tensor. In the presence of a uniform magnetic field $h_{z}$, both generate an effective staggered field $h_{x}$ perpendicular to the direction of $h_{z}$ [OA97, AO99]. These effects are captured by the isotropic Heisenberg chain with antiferromagnetic exchange coupling $J=1$ in a staggered transverse field as included in the model Hamiltonian in Eq. (4.13). In the following, this model is studied. For more details see Sec. 4.5. Note that the proof-of-principle calculations assume a larger value of $h_{x}$ than in real materials where the staggered field is usually only a few percent of the applied uniform field.

The dynamical spin structure factor $S_{z z}(k, \omega)$ is studied in the central region of the magnetization curve, i.e, $h_{z}=1$. In order to analyze the effect of these symmetry and integrability breaking interactions, MPS-based Chebyshev expansions are computed both for systems with strong interactions mimicked by a staggered field of $h_{x}=0.3$ and systems without DM interactions, i.e., $h_{x}=0$. Figure 5.7 presents the results for $S_{z z}(k, \omega)$ for $L=50$ sites and OBCs at temperatures of $T=0,0.5$, and 1 . Note that all temperatures are stated in units of $J$ in this chapter as $J=1$ is adopted for the calculations. An internal MPS bond dimension of $m=120$ states is retained. A separate calculation for each $k$ value is performed. At $T>0$, Chebyshev expansions of order $N=1500$ for a (nearly) uniform Gaussian broadening of $\eta \approx 0.13$ are obtained. As explained in Sec. 3.5.4, a fixed as well as frequency-independent resolution of the zero-temperature results are obtained by adapting the expansion order in dependence of $\omega$ in Figs. 5.7(a)(b). Unfortunately, in the published version of these two panels, i.e., Figs. 2(a)-(b) in Ref. [TMPH14], the expansion order of the results at $T=0$ is not adapted. Thus, the resolution of the Chebyshev expansions is frequency dependent and better than specified in Ref. [TMPH14]. Therefore, it differs from the resolution of the results at $T>0$. The reason is the frequency-dependent factor $\sqrt{1-\omega^{\prime 2}}$ in Eq. (3.95). This is corrected in the present Figs. 5.7(a)-(b) allowing for a direct comparison between $T=0$ and $T>0$ as the same frequency-independent broadening is used in both cases. This technical correction has no impact on the physical results in each panel.

First of all, the zero-temperature dynamical spin structure factor at $h_{x}=0$ is discussed. The result at $T=0$ obtained via an MPS-based Chebyshev expansion without the purification is shown in Fig. 5.7(a). For the discussion of these results, it is helpful to also include them at a higher resolution than the proof-of-principle results at finite temperature. These data using a smaller Gaussian broadening are shown in Fig. 5.8. It can be seen that the numerical results agree well with the analytical boundaries for the twospinon continua from Bethe ansatz [MTBB81]. Moreover, well-converged finite-size effects (FSEs) can be resolved for $L=50$. In the lower continuum, the oscillations are FSEs and decay in amplitude towards higher frequencies, most prominently at $k \approx 3 \pi / 4$. Note the tiny peak just above the lower boundary of the lower continuum at $k \approx 3 \pi / 8$ moving to higher frequencies with increasing $k$ and a similar branch visible in the upper two-spinon continuum which may be physical features. The high intensity for $k, \omega \rightarrow 0$ occurs due to spin conservation. For $h_{z}=1, S_{z z}(k, \omega)$ is gapless at $k=0$ and at $k \approx 3 \pi / 4$. This 


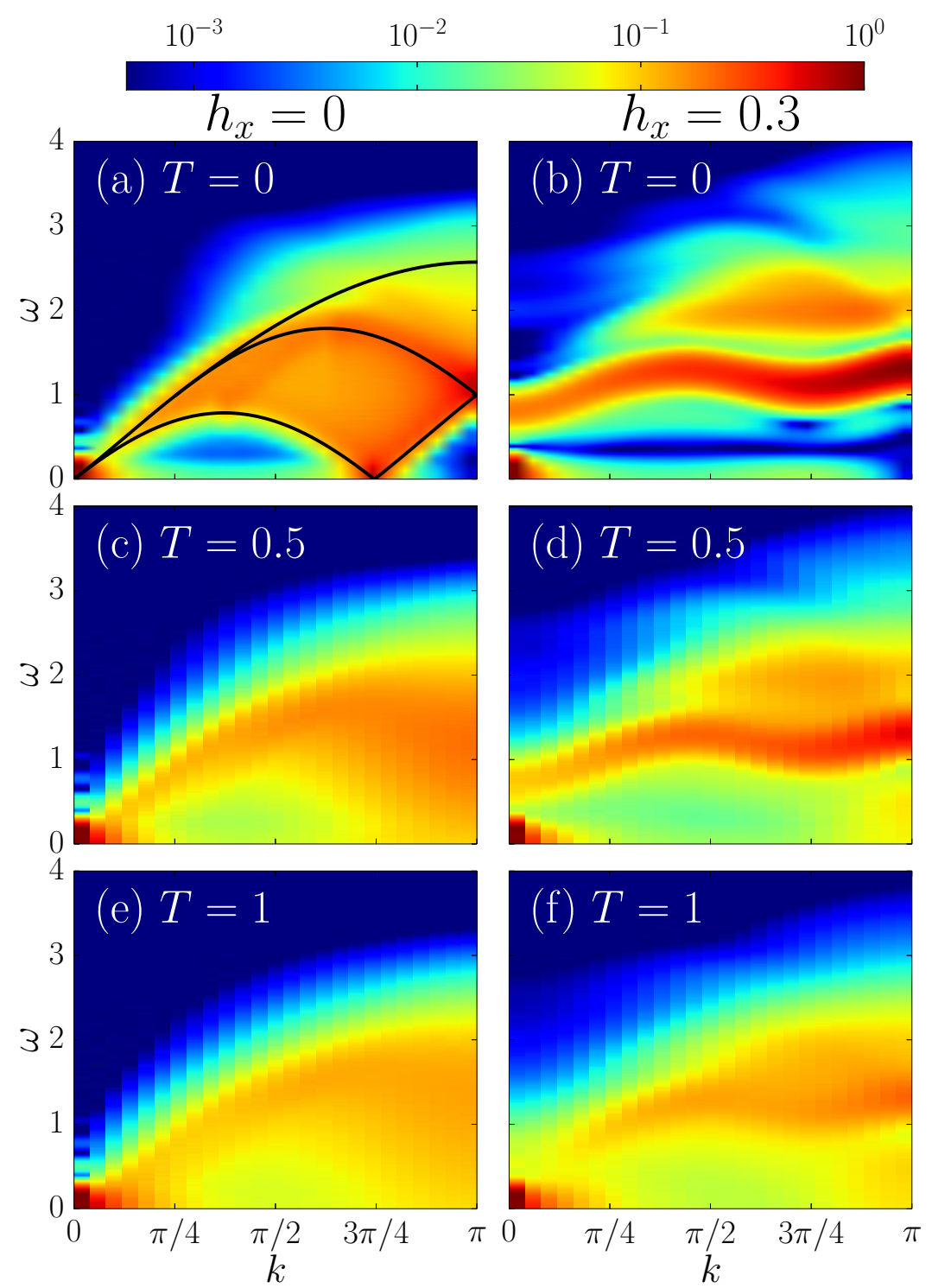

Figure 5.7: (Corrected version of Fig. 2 published in Ref. [TMPH14].) Longitudinal spin structure factor $S_{z z}(k, \omega)$ of an isotropic Heisenberg chain $(J=1)$ in a magnetic field $h_{z}=1$ obtained by the Chebyshev MPS method with approximate Gaussian broadening $\eta$. The left column shows results for $h_{x}=0(\eta \approx 0.13)$, and the right column for $h_{x}=0.3(\eta \approx 0.14)$. Top row: $T=0$. Central row: $T=0.5$. Bottom row: $T=1$.

is representative for a $T=0$ Luttinger liquid (LL) with Fermi momentum $2 k_{F} \approx 3 \pi / 4$ [Gia04].

It is now interesting to study how the LL behavior changes or even disappears by increasing the temperature. For example, in Refs. [FF10, $\left.\mathrm{BHM}^{+} 12\right]$, the 'melting' of a LL for a $t$ - $J$ chain with Kondo impurities and for $\mathrm{SU}(N)$ symmetric Hubbard systems, respectively, has been investigated numerically by considering spectral functions at finite (effective) temperatures. The authors of Ref. $\left[\mathrm{BHM}^{+} 12\right]$ used a quench extension of METTS and found the peak at $2 k_{F}$ to move with temperature to $k=\pi$ at $T \sim 0.2$ in their units of energy. The quantum Monte Carlo results of Ref. [GB09] indicate that at $T=0.25$ a feature in the vicinity of $2 k_{F}$ remains visible. Therefore, this system represents an interesting testing ground for proof-of-principle calculations using the Liouville-space 


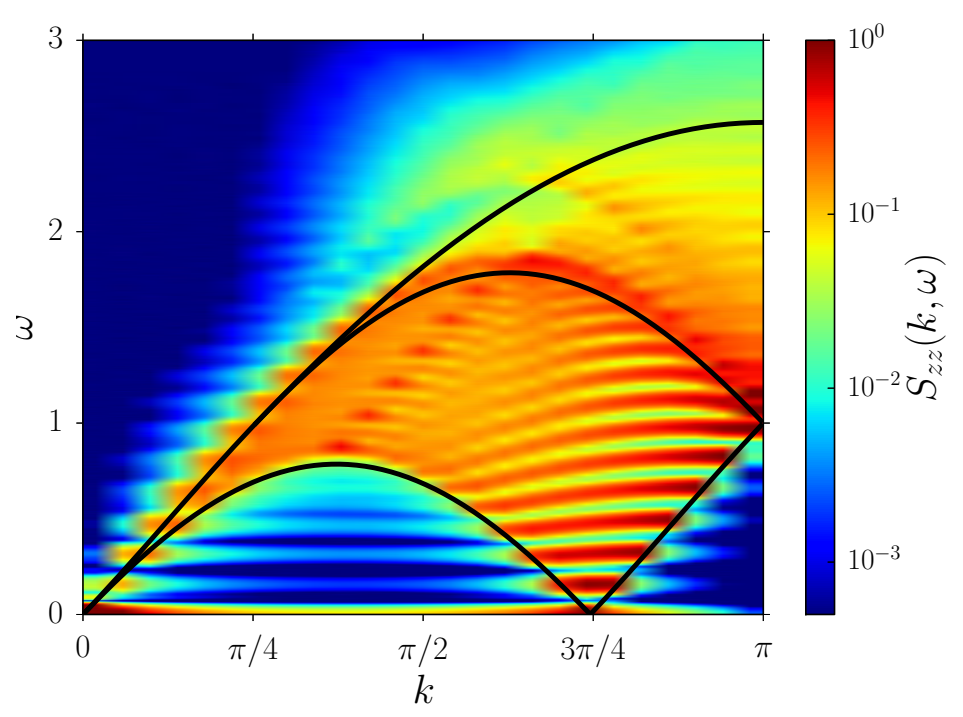

Figure 5.8: Zero-temperature results for the longitudinal spin structure factor $S_{z z}(k, \omega)$ of a Heisenberg chain in a magnetic field of $h_{z}=1$ obtained by the Chebyshev MPS method with uniform Gaussian broadening $\eta=0.025$. This figure shows the results of Fig. 5.7(a) at a higher resolution. This is for instance helpful to discuss finite-size effects. The solid lines represent the two-spinon continua from Bethe ansatz [MTBB81]. Note that $h_{x}=0$.

formulation and an MPS-based Chebyshev expansion. The finite-temperature results for $h_{x}=0$ are thus shown in Figs. 5.7(c) and 5.7(e). These calculations are in accordance with Ref. $\left[\mathrm{BHM}^{+} 12\right]$ since for $T=0.5$ the zero-temperature signal at $k \approx 3 \pi / 4$ is replaced by a broad distribution around $k=\pi$ in Fig. 5.7(c). Besides this tremendous redistribution of spectral weight, there is also strong temperature broadening and spectral intensity is found below the lower boundary of the two-spinon continuum at zero-temperature. Increasing the temperature to $T=1$ in Fig. 5.7(e) leads to further temperature broadening, but does not significantly alter the picture.

Now the effect of a staggered field of magnitude $h_{x}=0.3$ on $S_{z z}(k, \omega)$ is studied. Comparing Figs. 5.7(a) and 5.7(b), one identifies the opening of a field-induced gap at $T=0$ and the formation of a well-defined band. This is in agreement with the expectations from adding a DM term to the Heisenberg Hamiltonian since it causes the opening of gaps [OA97, $\mathrm{DDacR}^{+}$96]. Interestingly, increasing the temperature from $T=0$ to $T=0.5$ does not change the results significantly: A redistribution of the weights is obtained and the signals are smeared out, but in contrast to the $h_{x}=0$ case the qualitative features persist. Further increasing the temperature to $T=1$ leads to a stronger redistribution of the weights and eventually the band disappears.

Next, these features are studied in more detail in Fig. 5.9, which shows the temperature dependence of the longitudinal spin structure factor for $h_{x}=0.3$ at $k=49 \pi / 51$. Note that the published Fig. 3 in Ref. [TMPH14] corresponds to the present Fig. 5.9. However, here the resolution has been enhanced by subsequent calculations using the variational compression (see Sec. 3.5.4) instead of an SVD compression. This reproduction also allowed to correct the zero-temperature results. As explained for Figs. 5.7(a)-(b) above, the resolution specified for the $T=0$ data published Fig. 3 of Ref. [TMPH14] is wrong since its frequency dependence has been ignored. This is corrected in Fig. 5.9 where all curves have the same resolution over the shown frequency range. It now turns out that at $T=0.25$ there is only little thermal broadening of the peak at $\omega \approx 1.3$. Only further increasing 


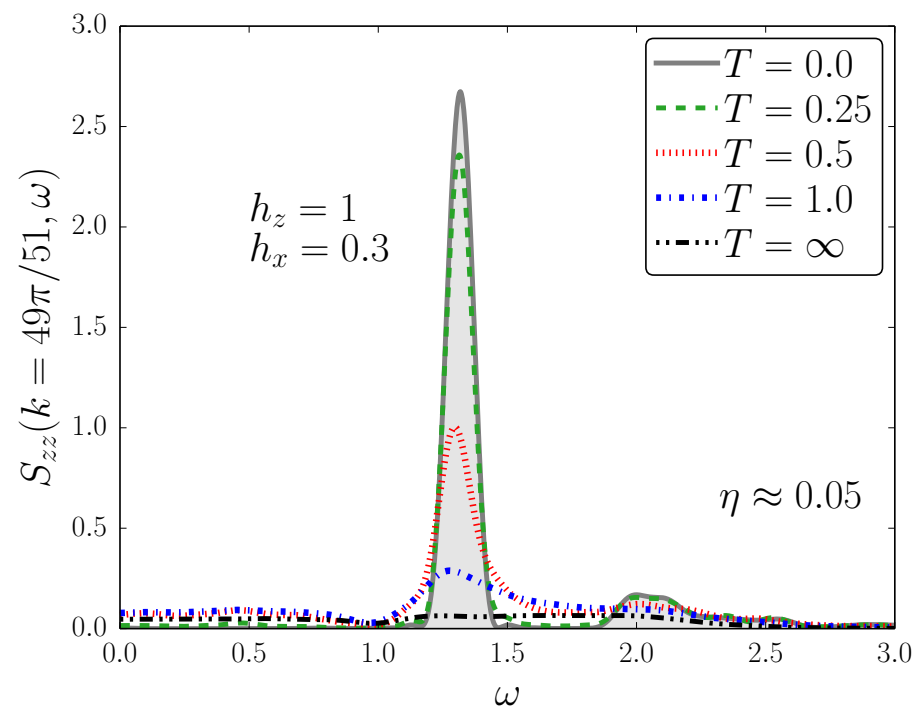

Figure 5.9: (Corrected version corresponding to Fig. 3 published in Ref. [TMPH14].) MPS results for the frequency dependence of $S_{z z}(k, \omega)$ at $k=49 \pi / 51$ of an isotropic Heisenberg chain $(J=1)$ with $L=50$ sites in a uniform magnetic field $h_{z}=1$ and an additional staggered field $h_{x}=0.3$ at temperatures $T=0,0.25,0.5,1$, and $\infty$.

temperature up to $T=0.5$ significantly broadens this feature. Also note the filling in of spectral weight into the gap at small frequencies when increasing the temperature, which is due to scattering. The signal corresponding to the excitations at high energies $(\omega \approx 2)$ starts to disappear for temperatures of the order of $T=0.5$. Even further increasing the temperature from $T=0.5$ to $T=1$ also significantly broadens the peak at $\omega \approx 1.3$, which corresponds to the band visible at $T=0$. Finally, at infinite temperatures, the curve is rather flat with only small features up to $\omega \approx 2$, where it slowly starts to decay to zero.

\section{\begin{tabular}{l|l} 
Continued fraction expansion results & 5.4
\end{tabular}}

This section is intended to demonstrate the flexibility of the Liouville-space formulation. The main content is directly adopted from the supplemental material of Ref. [TMPH14]. Although it is conceptually also possible to use other tensor network states [VCM08] than MPS in combination with the Liouvillian formulation, this aspect will not be changed in the following since efficient algorithms for the manipulation of such states are a subject of current research $\left[\mathrm{PBT}^{+} 15\right.$, Cor16]. The degree of flexibility studied here is related to the expectation that a variety of MPS methods can be applied using the Liouville space formulation. In the following, the finite-temperature spectral functions are obtained by a continued fraction expansion (CFE) generated by means of the Lanczos algorithm formulated with respect to the Liouville operator $\mathcal{L}$ (see Sec. 3.6.5).

In Fig. 5.10 the exact results for the longitudinal spin structure factor of an $X X$ chain with $L=30$ are compared to the CFE data. The exact results are obtained as described in Sec. 5.2.3, except that we adopt a Lorentzian broadening of $\eta=0.1$ on account of the $\mathrm{CFE}$. The agreement of the CFE results with the exact solutions is good as the relative deviations are always $\lesssim 10 \%$ (see below for more details). Comparing the CFE results for $L=30$ to the Chebyshev expansions for $L=50$ shown in Fig. 5.4, we find that the MPS bond dimension $m$ for a Chebyshev expansion can be chosen considerably smaller 


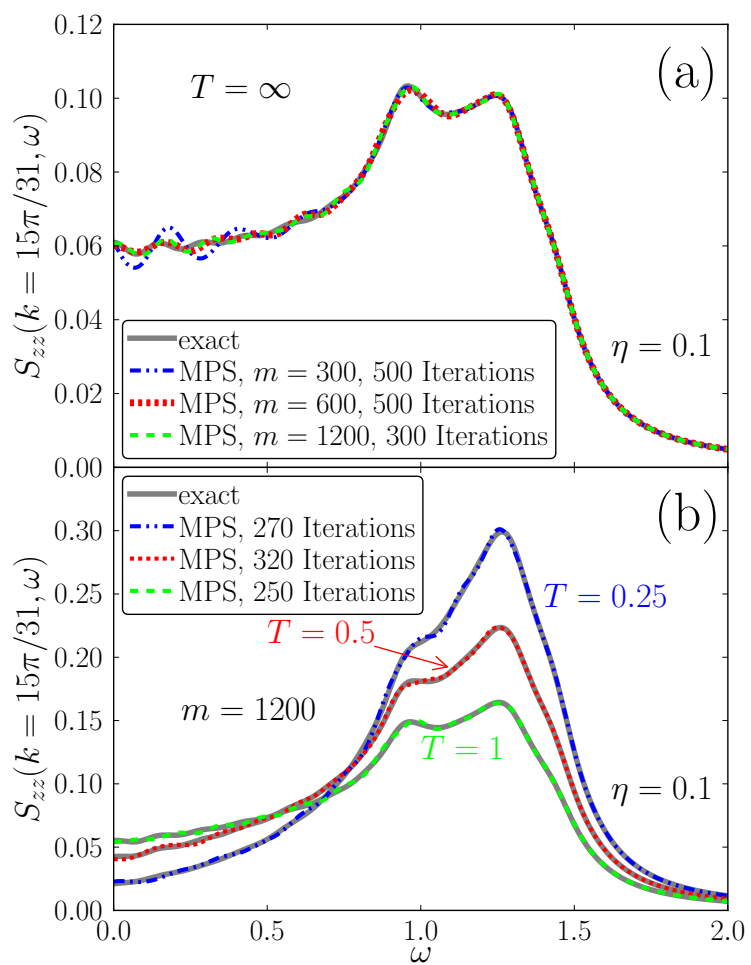

Figure 5.10: (Published in the supplemental material of Ref. [TMPH14].) Finite-temperature MPS calculations using a CFE for the dynamic spin structure factor $S_{z z}(k=15 \pi / 31, \omega)$ of an open $X X$ chain with $L=30$ compared to the exact solution. (a) At $T=\infty$ the accuracy is studied for different numbers $m$. (b) Temperature dependence of $S_{z z}(k=15 \pi / 31, \omega)$ compared to the exact solutions for $T=1,0.5$, and 0.25 (solid lines).

than for a CFE: When devising the Chebyshev expansion, visible deviations cannot be resolved in this plot for as small a value as $m=100$, whereas for the CFE in Fig. 5.10 we need to keep a substantially larger number $m \gtrsim 300$ to achieve a comparable accuracy at a still lower resolution. Moreover, the convergence properties of the Chebyshev expansion at finite temperatures also improve over those of the Lanczos algorithm. This becomes particularly clear in Fig. 5.10(a) which shows our CFE results for the longitudinal spin structure factor $S_{z z}(k=15 \pi / 31, \omega)$ at infinite temperature. Here, the absolute deviation of the CFE from the exact result ( $\lesssim 0.005$ for $m=300$ and $\lesssim 0.002$ otherwise) is largest in the center of the spectrum, i.e., at small frequency. This can be explained by the fact that the extremal eigenvalues at the edges of the spectrum converge best in the Lanczos algorithm.

In Fig. 5.10(b) the temperature dependence of $S_{z z}(k=15 \pi / 31, \omega)$ for $m=1200$ is depicted. Again, the CFE results fit the exact curves well down to temperatures as small as $T=0.25$. A detailed analysis reveals that the maximum relative deviation for $m=1200$ is $\sim 3.5 \%$ for $T=1, \sim 6 \%$ for $T=0.5$ and $\sim 8.5 \%$ for $T=0.25$ (the absolute deviations are $\lesssim 0.004$ in all cases). Thus, these errors in the spectral functions are mostly due to the CFE and can be lowered by devising a Chebyshev expansion.

In Fig. 5.11, we furthermore show the momentum-resolved spin structure factor of the isotropic Heisenberg chain in a uniform magnetic field $h_{z}$ with and without a staggered magnetic field $h_{x}$ at temperatures $T=0,0.5$, and 1 obtained via a CFE. All panels of this figure correspond to those of Fig. 5.7 featuring the Chebyshev results. The main features of the dynamical spin structure factor are also resolved by the CFE. However, 
in the Lanczos algorithm an MPS internal bond dimension of $m=500$ is retained for a chain of $L=30$ and at least 300 iterations are performed. This yields a well converged CFE with a Lorentzian broadening of $\eta=0.2$. In contrast, the Chebyshev expansion only requires $m=120$ states for $L=50$ at an even enhanced resolution.

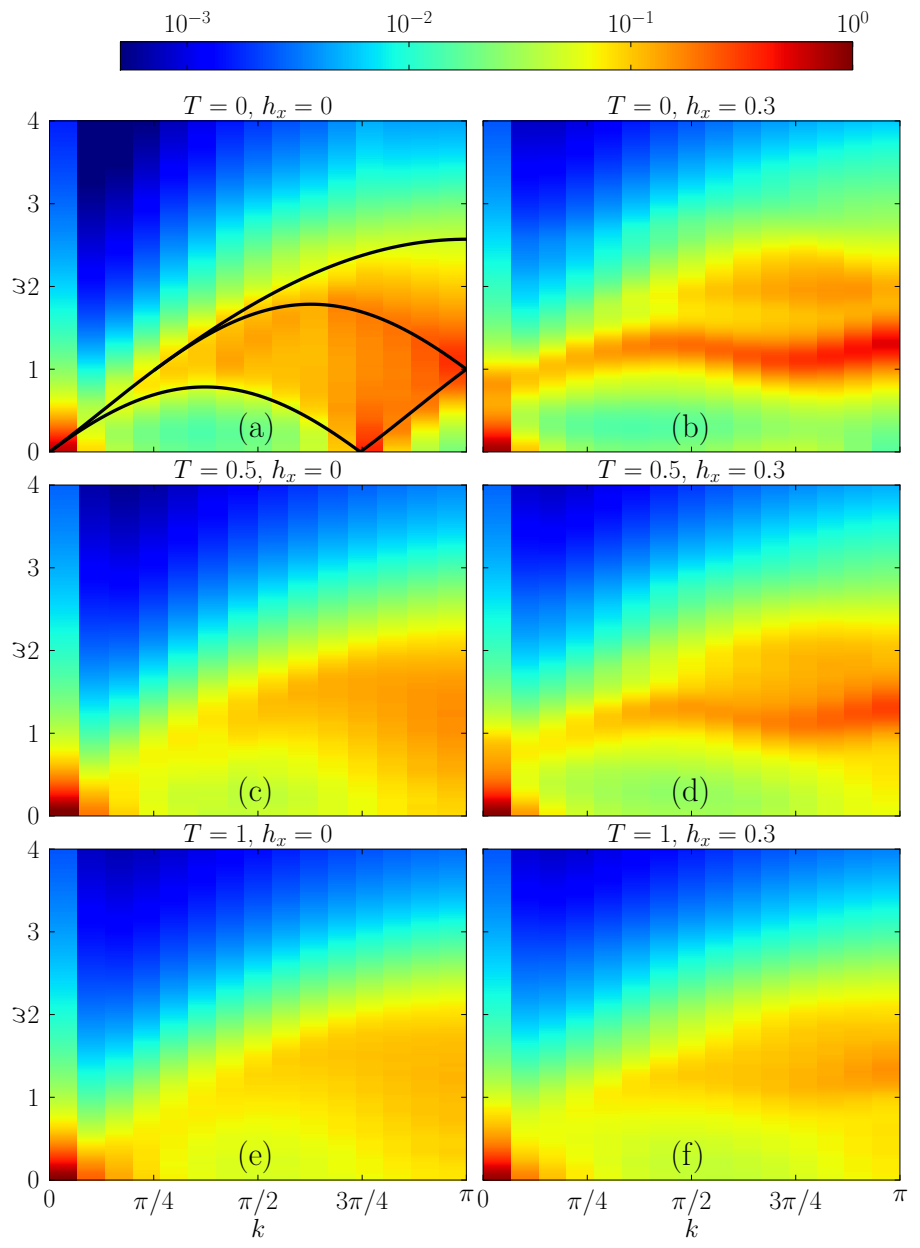

Figure 5.11: (Published in the supplemental material of Ref. [TMPH14].) Longitudinal spin structure factor $S_{z z}(k, \omega)$ of a Heisenberg chain with $L=30$ in a magnetic field $h_{z}=1$ obtained with the CFE approach $(\eta=0.2)$. The left column shows results for $h_{x}=0$, and the right column for $h_{x}=0.3$. Top row: $T=0$. Central row: $T=0.5$. Bottom row: $T=1$. 


\section{\begin{tabular}{l|r} 
Interim summary & 5.5
\end{tabular}}

In this chapter, results from an efficient and very accurate approach to compute finitetemperature spectral functions directly in the frequency domain are presented. The approach exploits a Liouville-space formulation of the dynamics. It was implemented via a Chebyshev expansion in an MPS framework. A comparison between proof-of-principle results for the longitudinal dynamical spin structure factor of the $X X$ model and exact results gives very good quantitative agreement at $T>0$. The high resolution allowed to observe the disappearance of the Luttinger-liquid ground state upon increasing temperature, see Sec. 5.3.2. In contrast, the effect of DM interactions and a staggered $g$ tensor leads to an opening of a field-induced gap and the formation of a band which both remain stable over a wide temperature range.

The flexibility of the Liouvillian formulation was demonstrated by showing additional results from an alternative CFE implementation in the previous section. For the systems considered in this chapter, the MPS Chebyshev method turned out to be more efficient. In particular, the two-spinon continua could be better resolved for even larger systems and smaller internal MPS bond dimensions. Thus, Chebyshev expansions will be used in the following chapters.

So far, the focus has been on proof-of-principle calculations, it is expected that the frequency resolution can be further increased by using a variational compression of the Chebyshev vectors after each iteration (see Sec. 3.5.4) instead of an SVD compression employed for the majority of the results shown in this chapter. The scope of the following two chapters is the application of this unbiased and efficient approach to models for quantum magnetism describing quasi-1D compounds. This will allow for a direct comparison to experimental results at finite temperatures, as obtained in electron spin resonance and inelastic neutron scattering experiments. 


\section{Dynamical properties of the sine-Gordon quantum spin magnet Cu-PM 6}

The content of this chapter is published as a regular article in Physical Review B [ $\left.\mathrm{THP}^{+} 16\right]$. It is therefore mainly adopted from the manuscript of Ref. [THP $\left.{ }^{+} 16\right]$. This manuscript was written primarily by me and revised together with the coauthors. I also performed the DMRG calculations. The exact diagonalization results shown in the present chapter and Figure 6.1 were contributed by Andreas Honecker. The ESR experiments have been conducted by Alexey Ponomaryov and Sergei A. Zvyagin at the Dresden High Magnetic Field Laboratory.

\section{\begin{tabular}{l|l} 
Introduction & 6.1
\end{tabular}}

The isotropic spin-1/2 Heisenberg chain with antiferromagnetic nearest-neighbor exchange coupling is a paradigmatic model for quantum magnetism. Due to the strong enhancement of quantum fluctuations on account of its low dimensionality, there is no long-range order at zero temperature. The ground state is a spin singlet and its elementary excitations are spinons carrying a fractional $S=1 / 2$. They are unbound and interact only weakly [FT81]. The dynamics of this model is governed by a gapless two-spinon continuum $\left[\mathrm{KMB}^{+} 97, \mathrm{CH} 06\right]$. A uniform magnetic field makes the soft modes of the excitation spectrum incommensurate $\left[\mathrm{MTBB} 81, \mathrm{SRB}^{+} 03\right]$, but leaves the spinon continuum gapless. Since the Heisenberg antiferromagnetic chain is in a critical phase, even small perturbations can significantly change its ground-state physics.

A typical perturbation in spin-chain materials is the presence of Dzyaloshinskii-Moriya interactions caused by spin-orbit coupling and/or a staggered $g$ tensor due to alternating crystal axes. In such cases, an applied magnetic field $H$ induces an effective transverse staggered field $h_{\text {stag }} \propto H$, which opens an energy gap $\propto H^{2 / 3}$ [OA97, AO99]. There are several realizations of such quasi-1D materials, for example, copper benzoate $\left(\mathrm{Cu}\left(\mathrm{C}_{6} \mathrm{D}_{5} \mathrm{COO}\right)_{2} \cdot 3 \mathrm{D}_{2} \mathrm{O}\right)\left[\mathrm{DDacR}^{+} 96, \mathrm{DHR}^{+} 97, \mathrm{ANI}^{+} 00, \mathrm{ANI}^{+} 03\right]$, copper pyrimidine dinitrate $\left(\left[\mathrm{PM} \cdot \mathrm{Cu}\left(\mathrm{NO}_{3}\right)_{2} \cdot\left(\mathrm{H}_{2} \mathrm{O}\right)_{2}\right]_{n}, \mathrm{PM}=\right.$ pyrimidine; or shortly $\left.\mathrm{Cu}-\mathrm{PM}\right)\left[\mathrm{INN}^{+} 97, \mathrm{YIA}^{+} 01\right.$, $\left.\mathrm{FAG}^{+} 00, \mathrm{ZKKF} 04, \mathrm{ZKKF} 05, \mathrm{ZCO}^{+} 11\right], \mathrm{Yb}_{4} \mathrm{As}_{3}\left[\mathrm{KIM}^{+} 01\right], \mathrm{CuCl}_{2} \cdot 2$ (dimethylsulfoxide) $\left[\mathrm{KCB}^{+} 04, \mathrm{KBC}^{+} 05\right]$, and $\mathrm{KCuGaF}_{6}\left[\mathrm{UTO}^{+} 09, \mathrm{UTO}^{+} 12, \mathrm{UTK}^{+} 15\right]$.

The low-energy degrees of freedom of these materials can be effectively described in the framework of the quantum sine-Gordon field theory (see Sec. 4.5.2). One central approximation of this approach is that the field-dependent parameters such as the coupling constant $\beta(H)$ in the sine-Gordon model need to be determined in the absence of the staggered field. The quantum sine-Gordon model is exactly solvable [Lut76, ZZ79, BT79, Kor79], so that many experimental observables can be evaluated for these sine-Gordon quantum magnets. This includes static properties such as the specific heat [Ess99] and the field dependence of the excitation energies [OA97, ET98, AO99]. Very important progress has 
been made in the prediction of dynamical properties. Nevertheless, they are accessible only for a restricted set of wave vectors. For instance, the dynamical magnetic susceptibilities can be calculated at the antiferromagnetic wave vector $q=\pi$ using the formfactor method [ET98]. This approach has also been used to obtain the dynamical spin structure factor for both the isotropic Heisenberg chain [EFH03] and anisotropic $X X Z$ Heisenberg antiferromagnets [KE09] in a uniform longitudinal and a transverse staggered field. Nevertheless, the sine-Gordon theory does not fully capture the physics of highenergy bound-spinon states observed in neutron scattering $\left[\mathrm{KBC}^{+} 05\right]$ and its predictions are limited to the range of small to moderate fields. Beyond this, the density-matrix renormalization group (DMRG) [Whi92, Whi93, PWKH99, Sch05a] has provided new insights into the field dependence of a few low-lying excitations up to strong magnetic fields $\left[Z_{W X}^{+} 03, \mathrm{LCZ}^{+} 05, \mathrm{LCW} 06\right]$. Furthermore, it has been shown that DMRG calculations for the lowest excitation are in agreement with ESR experiments probing the field dependence of the excitation gap at very strong magnetic fields $\left[\mathrm{ZCO}^{+} 11\right]$. For small systems, there exist a few numerical results for dynamical properties of sine-Gordon quantum magnets, see, e.g., Refs. [KBC $\left.{ }^{+} 05\right]$ and [IE03].

In the present work, these systems are revisited by means of a detailed and systematic study based on DMRG and exact diagonalization (ED) [NM05, San10, HW09] calculations for momentum- and frequency-resolved response functions at zero and at finite temperature. This gives direct access to relevant dynamical quantities probed in electron spin resonance (ESR) [OA02, Zvy12] or neutron scattering allowing for the study of new features emerging upon raising the temperature. In the employed approach, the DMRG results for the spectral functions are computed directly in the frequency domain via a Chebyshev expansion of the dynamical correlation function (see Sec. 3.5.4) using matrix product states (MPS) [Sch11]. At finite temperatures this is done in the framework of a Liouville-space formulation for the dynamics of the purified finite-temperature density operator, see Sec. 3.6. This combination of methods is used to investigate various aspects of previous predictions and findings with a high resolution, and to make a prediction for the evolution of intensities of the spectral functions with temperature.

At $T=0$, the ESR resonance modes and their intensities are studied for a wide range of the applied magnetic field and at higher frequency. Although the predictions by sineGordon field theory are in very good agreement with many of the resonance frequencies in ESR experiments, a systematic deviation for the single-soliton resonance probed in $\mathrm{Cu}-\mathrm{PM}$ [ZKKF04] has been observed. In this chapter, the discrepancy can be resolved with the help of DMRG results for the dynamical spin structure factor.

As ESR experiments on Cu-PM [ZKKF04, ZKKF05, ZCO $\left.{ }^{+} 11\right]$ and $\mathrm{KCuGaF}_{6}$ [UTO $\left.{ }^{+} 09\right]$ also probed excitations which cannot be explained in the bulk sine-Gordon theory, Furuya and Oshikawa studied boundary and impurity effects in sine-Gordon quantum magnets [FO12]. Using a boundary sine-Gordon field theory approach, they found that there is only one type of boundary bound state (BBS) for the soliton, antisoliton, and the first breather, which is found below the bulk gap. The energy of this BBS is also known from DMRG calculations restricted to low-lying excitations [ $\left.\mathrm{LCZ}^{+} 05, \mathrm{LCW} 06\right]$. According to the boundary field theory, there are even more predictions for boundary resonances at $T=0$. However, the exact intensities of these excitations have not been determined, yet. In this work it is found that, except for the BBS, none of the boundary resonances is clearly observable in the DMRG results for the spectral functions.

At $T>0$, Ref.[FO12] also expects additional thermally induced transitions between the sine-Gordon excitations. Since a few interbreather excitations were experimentally 
observed in $\mathrm{KCuGaF}_{6}\left[\mathrm{UTO}^{+} 09\right]$, one central goal of this chapter is the observation of thermally-induced transitions between breathers in the material $\mathrm{Cu}-\mathrm{PM}$. To this end, both numerical calculations and accompanying ESR experiments are performed for a wide range of the applied magnetic field. It is found that finite temperature can lead to excitations between the elementary breatherlike excitations of the sine-Gordon field theory at $T=0$. The evolution of their intensities with temperature will be studied.

The chapter is organized as follows. Section 6.2 provides information about the compound $\mathrm{Cu}-\mathrm{PM}$ and reviews further experimentally observable excitations in sine-Gordon magnets. The relevant contributions probed in ESR experiments on sine-Gordon quantum magnets are reviewed in Sec. 6.3. Then the details and parameters of the numerical calculations are briefly presented in Sec. 6.4. In Sec. 6.5, the zero-temperature ESR modes in the material $\mathrm{Cu}-\mathrm{PM}$ and their intensities are studied and compared to previous experiments. Section 6.6 is focused on thermally activated transitions between excited states and includes both numerical and experimental results. Finally, the main results and conclusions of the chapter are summarized in Sec. 6.7.

\section{\begin{tabular}{l|l} 
The compound Cu-PM & 6.2
\end{tabular}}

\section{\begin{tabular}{l|l} 
Crystal structure and effective model & 6.2 .1
\end{tabular}}

Quasi-1D spin systems such as Cu-PM $\left[\mathrm{INN}^{+} 97, \mathrm{YIA}^{+} 01, \mathrm{FAG}^{+} 00, \mathrm{WRC}^{+} 03, \mathrm{ZKKF} 04\right.$, $\left.\mathrm{WWS}^{+} 05, \mathrm{ZKKF} 05, \mathrm{DWK} 07, \mathrm{ZCO}^{+} 11\right]$ and copper benzoate $\left[\mathrm{DDacR}^{+} 96, \mathrm{DHR}^{+} 97\right.$, $\mathrm{ANI}^{+} 00, \mathrm{ANI}^{+}$03] possess alternating crystal axes giving rise to staggered DzyaloshinskiiMoriya (DM) interactions and an alternating $g$ tensor. The crystal structure of Cu-PM $\left[\mathrm{INN}^{+} 97, \mathrm{YIA}^{+} 01, \mathrm{FAG}^{+} 00\right]$ is illustrated in Fig. 6.1.

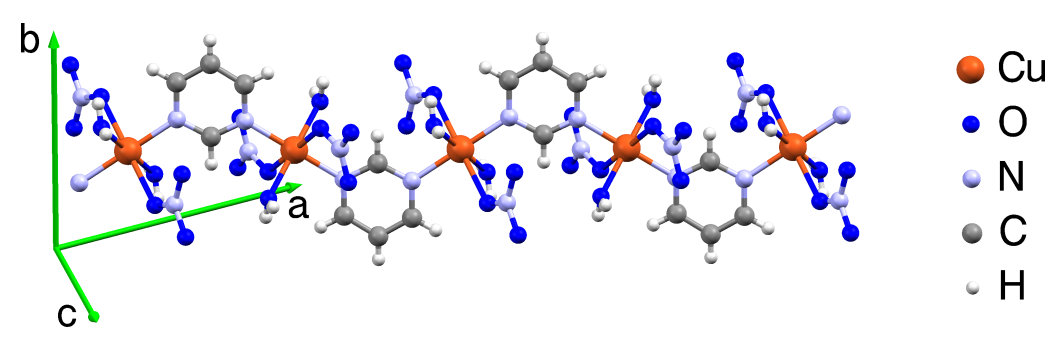

Figure 6.1: (Taken from Ref. $\left[\mathrm{THP}^{+} 16\right]$.) Structure of $\mathrm{Cu}^{-}$ PM: chain of staggered $\mathrm{Cu}$ coordination octahedra linked by pyrimidine rings (running along the $a-c$ diagonal). The magnetic interaction $J$ is mediated by a $\mathrm{Cu}-\mathrm{N}-\mathrm{C}-\mathrm{N}-\mathrm{Cu}$ superexchange pathway. (This figure was created using the program Mercury $3.3\left[\mathrm{MBC}^{+} 08\right]$.)

As reviewed in Sec. 4.5, the DM interactions can be eliminated by a staggered rotation of the spin operators yielding an effective transverse staggered field $h_{\text {stag }}=h_{x}$ which is chosen along the $x$ direction. Its strength is proportional to the uniform field applied in the longitudinal direction, $h_{x}=c h_{z}$. For the compound $\mathrm{Cu}-\mathrm{PM}$ treated here, one has $J / k_{\mathrm{B}}=36 \mathrm{~K}$ and $|\mathbf{D}|=0.139 J\left[\mathrm{FAG}^{+} 00\right]$, so that the condition $|\mathbf{D}| \ll J$ is fulfilled, and the effective model Hamiltonian in Eq. (4.13) is expected to be valid for all strengths of the magnetic field considered. The parameter $c$ is a material constant that determines 
the magnitude of the staggered transverse field. When the magnetic field is applied along the direction in which the maximal staggered magnetization is assumed, the material parameters $c=0.08$ and $c=0.083$ were determined for Cu-PM [ZKKF04, ZKKF05].

\section{\begin{tabular}{l|l} 
Elementary excitations & 6.2 .2
\end{tabular}}

The zero-temperature elementary excitations at low energies of the model Hamiltonian (4.13) for Cu-PM can be effectively described by the quantum sine-Gordon model as long as $h_{\text {stag }} \ll J$. This model as well as its bulk excitations have already been reviewed in Sec. 4.5.2. A schematic sketch of the low-energy modes, such as solitons, antisolitons, and their bound states, which are called breathers, is given in Fig. 4.3. In the following, further excitations predicted by sine-Gordon field theory are briefly reviewed and their observation in ESR experiments on $\mathrm{Cu}-\mathrm{PM}$ or $\mathrm{KCuGaF}_{6}$ is summarized.

In the sine-Gordon magnets $\mathrm{Cu}-\mathrm{PM}\left[\mathrm{ZKKF} 04, \mathrm{ZCO}^{+} 11\right]$ and $\mathrm{KCuGaF}_{6}\left[\mathrm{UTO}^{+} 09\right]$, there are experimentally observed ESR resonance modes which cannot be accounted for by the bulk sine-Gordon theory for an infinite system which has been discussed so far. Pursuing a boundary sine-Gordon field theory approach, Furuya and Oshikawa found that for the soliton, antisoliton, and the first breather there is only one identical boundary bound state (BBS) [FO12]. This BBS is found below the bulk gap and its mass is given by

$$
M_{\mathrm{BBS}}=M_{S} \sin (\pi \xi) .
$$

Here $M_{S}$ is the soliton mass from Eq. (4.15). Furthermore, Furuya and Oshikawa argued that additional boundary resonances predicted from their theory can be assigned [FO12] to unexplained modes in the materials $\mathrm{Cu}-\mathrm{PM}$ [ZKKF04, $\left.\mathrm{ZCO}^{+} 11\right]$ and $\mathrm{KCuGaF}_{6}$ $\left[\mathrm{UTO}^{+} 09\right]$.

Since this study is interested in finite-temperatures excitations, note that interbreather transitions at frequencies $M_{n}-M_{m}(n>m)$ are possible, and Ref. [UTO ${ }^{+} 09$ ] reports to have observed these excitations for the material $\mathrm{KCuGaF}_{6}$ in pulsed-field ESR experiments, even though the measurements were performed at very low temperatures $(T / J \approx 0.005)$. Moreover, there is the field theoretical expectation [FO12] for additional finite-temperature resonances at $\left|E_{S}-M_{n}\right|$, which are soliton-breather transitions. These excitations will be reconsidered in Sec. 6.6.2.

\section{\begin{tabular}{l|l} 
ESR and mixing of components & 6.3
\end{tabular}}

In ESR [OA02] experiments a linearly polarized electromagnetic wave is coupled to the $q=0$ component of the total spin operator $S^{\alpha}=\sum_{i} S_{i}^{\alpha}$. In the Faraday configuration the radiation is polarized perpendicular to the applied magnetic field in the $z$ direction, i.e., $\alpha \perp z$. Within linear response theory [Pru14], the absorption intensity

$$
I(\omega)=\frac{\pi f_{0}^{2}}{2} \omega \chi_{\text {phys }}^{\prime \prime}(q=0, \omega)
$$

is proportional to the imaginary part $\chi^{\prime \prime}$ of the dynamical magnetic susceptibility. The amplitude of the electromagnetic wave is denoted by $f_{0}$, which is set to $f_{0}=1$ for the 


\begin{tabular}{c|c|c}
\hline \hline & bulk & boundary \\
\hline$\chi_{+-}^{\prime \prime}(q=\pi, \omega)$ & $\omega=M_{n}, M_{n}+M_{m}$ & $\omega=M_{\mathrm{BBS}}, M_{n}+M_{\mathrm{BBS}}$ \\
\hline$\chi_{+-}^{\prime \prime}(q=0, \omega)$ & $\omega=E_{S}, E_{S}+M_{n}$ & $\omega=E_{n}=\sqrt{M_{n}^{2}+h_{z}^{2}}$ \\
& & $E_{S}+M_{\mathrm{BBS}}, E_{n}+M_{\mathrm{BBS}}$ \\
\hline \hline
\end{tabular}

Table 6.1: (Table adapted from Ref. [FO12]) Typical resonance modes from bulk resp. boundary sine-Gordon field theory at $T=0$. Note that $\chi_{z z}^{\prime \prime}(q=\pi, \omega)$ contains the same resonances as $\chi_{+-}^{\prime \prime}(q=0, \omega)$.

calculations. Due to the staggered rotation of the spin operators performed to eliminate the DM interaction in Eq. (4.12), the dynamical susceptibility $\chi_{\text {phys }}$ relevant for the experiments has contributions from both uniform $(q=0)$ and staggered $(q=\pi)$ susceptibilities calculated for the effective model in Eq. (4.13) [FO12]:

$$
\begin{aligned}
& \chi_{\text {phys }}^{\prime \prime}(q=0, \omega) \sim \chi_{+-}^{\prime \prime}(q=0, \omega) \\
& \quad+\left(\frac{D_{z}}{J}\right)^{2} \chi_{+-}^{\prime \prime}(q=\pi, \omega)+\left(\frac{D_{\perp}}{J}\right)^{2} \chi_{z z}^{\prime \prime}(q=\pi, \omega) .
\end{aligned}
$$

The mixing is determined by $D_{z}$ and $D_{\perp}$ which are the components of the DM vector $\mathbf{D}$ parallel and perpendicular to the external magnetic field pointing in the $z$ direction. Note that in ESR experiments on sine-Gordon magnets only excitations occurring at $q=0$ and $q=\pi$ for effective model in Eq. (4.13) can be probed [Zvy12]. Therefore, the dispersion branch linked to the soliton $S$ and the antisoliton $\bar{S}$ at incommensurate wave vectors is probed at the two experimentally accessible momenta $q=0$ and $q=\pi$, see Fig. 4.3. In Eq. (6.3), $\chi_{+-}^{\prime \prime}\left(\chi_{z z}^{\prime \prime}\right)$ denotes the imaginary part of the transverse (resp. longitudinal) dynamical susceptibility. The closely related dynamical spin structure factor then reads

$$
S_{\alpha \gamma}(q, \omega)=\frac{1}{\pi} \frac{\chi_{\alpha \gamma}^{\prime \prime}(q, \omega)}{1-e^{-\omega /\left(k_{\mathrm{B}} T\right)}} .
$$

Moreover, $k_{\mathrm{B}}=1$ and $\hbar=1$ are adopted in the calculations. It is known that the longitudinal susceptibility $\chi_{z z}^{\prime \prime}(q=\pi, \omega)$ contains the same resonances as the transverse contribution $\chi_{+-}^{\prime \prime}(q=0, \omega)$ [FO12]. That is why in particular $\chi_{z z}^{\prime \prime}(q=\pi, \omega)$ is considered in order to study the corresponding excitations since their intensity is enhanced in this component of the dynamical susceptibility. Table 6.1 gives an overview of the most relevant bulk and the predicted boundary excitations in the different components of the dynamical susceptibility at $T=0$ [FO12].

\section{\begin{tabular}{l|l} 
Numerical details & 6.4
\end{tabular}}

\section{DMRG calculations}

At $T=0$, the dynamical response functions are calculated in the frequency domain by MPS-based Chebyshev expansions (see Eq. (3.90)), as explained earlier in Sec. 3.5.4. If not specified differently, all spectral functions exhibit a uniform as well as finite Gaussian broadening $\eta$, which is achieved by adapting the expansion order as a function of 
frequency, cf. Eq. (3.95). After each Chebyshev iteration, the new Chebyshev state is variationally compressed to an MPS with smaller maximal matrix dimensions $m$. The accuracy is controlled by specifying $m$. If not stated otherwise, $m=250$ is used in order to evaluate the spectral line shape at $T=0$ corresponding to a compression error of $\epsilon_{\text {compr }} \sim 10^{-5}$, see Eq. (3.94).

At $T>0$, the finite-temperature MPS approach developed in this thesis is used (see Sec. 3.6). Thus, the dynamical susceptibilities are computed directly in the frequency domain by exploiting a Liouville-space formulation. Here the method is implemented as a Chebyshev expansion with respect to the linearly rescaled Liouville operator as explained in Sec.3.6.4. For the evaluation of finite-temperature spectral functions in Sec.6.6, a maximal matrix dimension of up to $m=300$ is used in order to enforce a compression error of $\epsilon_{\mathrm{compr}} \lesssim 10^{-3}$.

\section{Exact diagonalization $\mid 6.4 .2$}

In this chapter, exact diagonalization [NM05, San10] results for the dynamical correlation functions are presented as well. The corresponding calculations have been performed by Andreas Honecker. Periodic boundary conditions (PBCs) are adopted, in order to exploit the translation invariance of the model in Eq. (4.13). For small systems, a full diagonalization of the Hamiltonian was achieved for $L \leq 16$ in Ref. [WWS ${ }^{+} 05$ ]. This could be extended up to $L=18$ for the present purposes. In order to treat even larger systems, the Lanczos method (see Sec. 3.2) is used in order to compute low-lying eigenstates $\left|E_{n}\right\rangle$ and the corresponding eigenvalues $E_{n}$. To obtain a large number of eigenvectors, the procedure from Ref. [HW09] is applied to remove the so-called ghosts occurring in the course of the Lanczos recursion. As not all eigenstates are accessible in this manner, only a limited number of terms in the double sum in Eq. (2.29) can be taken into account. Thus, reliable results for the finite-temperature spectral functions can only be obtained at low temperature and small frequency. For $L=20$, about 10000 terms are calculated, whereas for $L=24$ roughly 2000 terms are kept. Since the individual eigenvalues and states are accessible, the Gaussian broadening as well as the temperature is specified in the post-processing stage. This grants results for different (low) temperatures without a completely new calculation.

\section{\begin{tabular}{l|l} 
Zero-temperature results & 6.5
\end{tabular}}

For the numerical study of the ESR modes and their intensities, the value of $c$ is a crucial model parameter as it determines the magnitude of the staggered transverse field $h_{x}=c h_{z}$. For instance, in $\mathrm{KCuGaF}_{6}$ the parameter $c$ assumes a value of $c=0.178$ in the direction of the maximal staggered magnetization $\left[\mathrm{UTO}^{+} 09\right]$. In copper benzoate $\left[\mathrm{DDacR}^{+} 96, \mathrm{DHR}^{+} 97, \mathrm{ANI}^{+} 00, \mathrm{ANI}^{+} 03\right]$ and dimethylsulfoxide $\mathrm{CuCl}_{2}\left[\mathrm{KCB}^{+} 04\right.$, $\mathrm{KBC}^{+} 05$ ] the values are $c=0.065$ and $c=0.075$, respectively. This is a typical order of magnitude that also applies to $\mathrm{Cu}-\mathrm{PM}$. When the magnetic field is applied along the $c^{\prime \prime}$ direction, in which the maximal value of the staggered magnetization is assumed, the material parameters $c=0.08$ and $c=0.083$ were determined for Cu-PM [ZKKF04, ZKKF05]. 
Except for Sec. 6.5.3, where $c=0.08$ is considered for a direct comparison to the experiments in Ref. [ZKKF04], the value $c=0.083$ is adopted for the numerical calculations at $T=0$.

\section{BBS and breather excitations}

First, the BBS and the breather excitations are studied in the transverse staggered susceptibility $\chi_{+-}^{\prime \prime}(q=\pi, \omega)$. Figure $6.2($ a) shows the DMRG results for the related ESR intensity for $h_{z}=1$ and $c=0.083$. The first three breather excitations B1-B3 are clearly observed for different system sizes $L$. Since the DMRG calculations are performed for open boundary conditions (OBCs), the BBS observed in earlier DMRG studies $\left[\mathrm{LCZ}^{+} 05\right.$, LCW06] and finally identified as such in a boundary sine-Gordon field theory [FO12] is found slightly below B1 in the bulk gap. The masses of the elementary excitations from the sine-Gordon theory are included as vertical lines in Figs. 6.2(a)-(b). The peak positions are in good agreement with the predictions. Moreover, the intensity decreases for the heavier quasiparticles B2 and B3. Figure 6.2(b) shows the finite-size dependence of the BBS and B1 at an enhanced resolution $(\eta=0.006)$. Two interesting observations are that the position of the B1 peak seems to converge towards the field theoretical value, and that the BBS intensity decreases with increasing system size. To check this, finite-size analyses using a fitting function of the form $f(x)=A+B x^{\gamma}$ are performed, where $x=1 / L$. In Fig. 6.2(c) the analysis confirms that the $L \rightarrow \infty$ extrapolation of the B1 peak position agrees with the field theoretical breather mass $M_{1}$, which is plotted as the horizontal solid line. Furthermore, a finite-size scaling of the integrated peak intensity is performed, which is independent of the broadening, both for the BBS in Fig. 6.2(d) and for B1 in Fig. 6.2(e). Here the best agreement was achieved by setting the exponent $\gamma=1$. The peak intensity of the BBS scales close to zero, whereas the intensity
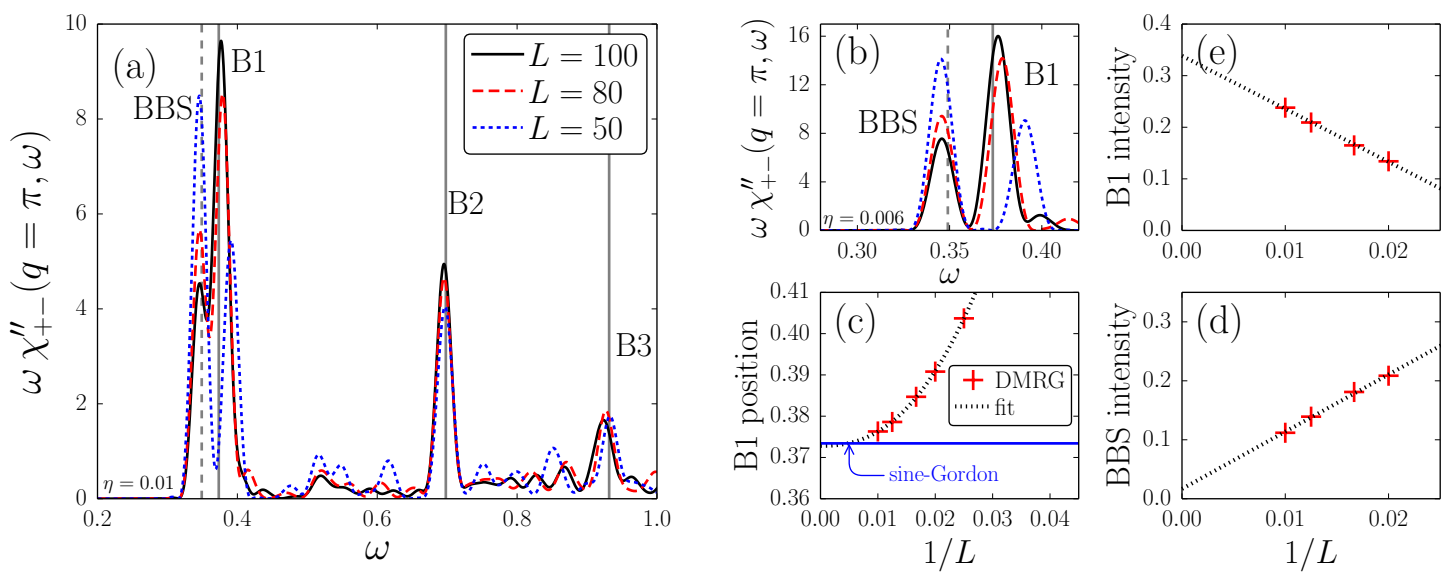

Figure 6.2: (Published in Ref. [THP $\left.{ }^{+} 16\right]$.) Zero-temperature DMRG results for the transverse staggered contribution to the ESR intensity at $h_{z}=1(J=1, c=0.083)$ complemented with values from the sine-Gordon theory (vertical lines): (a) Observation of the BBS and three breathers B1-B3 in the intensity $\sim \omega \chi_{+-}^{\prime \prime}(q=\pi, \omega)$ for various system sizes $L$ and a Gaussian broadening of $\eta=0.01$. (b) Zoom-in on BBS and B1 at higher resolution $(\eta=0.006)$. (c)-(e) Finite-size scaling analyses for the mass $M_{1}$ of B1 (c), and the integrated peak intensity of the BBS (d) as well as B1 (e). 
of B1 extrapolates to a finite value in the thermodynamic limit. Thus, the expectations for a boundary resp. bulk excitation are met.

As a next step, the frequency-field dependence of the breather excitations B1, B2, and B3 is determined from the DMRG results for the ESR spectral function. The peak positions for B1 and B3 are obtained from the absorption intensity $\sim \omega \chi_{y y}^{\prime \prime}(q=\pi, \omega)$ for $L=80$ which is plotted as a function of the magnetic field in Fig. 6.3(a). For this calculation, $m=150$ DMRG states are kept corresponding to $\epsilon_{\text {compr }} \sim 10^{-4}$ at small fields and $\epsilon_{\text {compr }}<10^{-5}$ for $h_{z}>1$. Since an MPS-based Chebyshev expansion of order $N=6000$ is used and the spectral width becomes larger for increasing fields, the Gaussian broadening included in these results depends both on the frequency and the field, i.e. $\eta\left(\omega, h_{z}\right)$. As an example, the broadening ranges from $\eta(\omega=0)=0.004$ to $\eta(\omega=3)=0.01$ at $h_{z}=1$ in this case. The frequency of $\mathrm{B} 2$ is determined from the ESR intensity $\sim \omega \chi_{+-}^{\prime \prime}(q=\pi, \omega)$, which is shown in Fig. A.1(a) in Appendix A.1. In Fig. 6.3(b), DMRG results for the breather resonances are compared to sine-Gordon predictions for an infinite system and exact diagonalizations (ED) of a system with $L=28$ sites. For B1, the DMRG results show deviations towards small magnetic fields, since in this field regime finite-size effects (FSEs) are gradually enhanced. However, this has been understood by the finitesize analysis in Figs. 6.2(b)-(c). Since the ED calculations are performed with periodic boundary conditions (PBCs), FSEs are less severe in the ED data. Interestingly, the DMRG results for B2 and B3 show a weaker finite-size dependence than for B1. In this figure one can also assess the limits of the field theoretical description which is based on the limit of a small $h_{x}$ : Towards high fields, $h_{z} \geq 1.3$, the description by the field theory breaks down the earlier the heavier the mass of the breather excitation is.

Moreover, it is interesting to study the evolution of the field-induced gap up to strong magnetic fields, i.e., beyond the realm of sine-Gordon physics. In Fig. 6.3(c), the frequencyfield dependence of the BBS extracted from the spectral function for $L=80$ in Fig. 6.3(a) is compared to DMRG results from Ref. $\left[\mathrm{ZCO}^{+} 11\right]$. Note that the previously published data for the lowest excitation energy computed by a multi-target DMRG approach for $L=200$ and OBCs perfectly coincidence with the present results for the BBS. Furthermore, the BBS is observed as a weak feature of the absorption intensity $\sim \omega \chi_{z z}^{\prime \prime}(q=\pi, \omega)$ in Fig. 6.5(a) below. Besides the BBS, Fig. 6.3(c) also includes results for B1. Interestingly, the two excitations merge into one single excitation close to the saturation field. In the fully spin-polarized phase at high fields, the elementary excitations are magnons and the gap is proportional to $h_{z}-h_{z}^{\text {sat }}$, where $h_{z}^{\text {sat }}$ is the saturation field $\left[\mathrm{ZCO}^{+} 11\right]$. Furthermore, the two-magnon continuum and in particular its lower boundary are clearly visible in Fig. 6.3(a). Again, note that the resolution becomes worse towards higher fields and frequencies, since the expansion order is kept fixed at $N=6000$. 

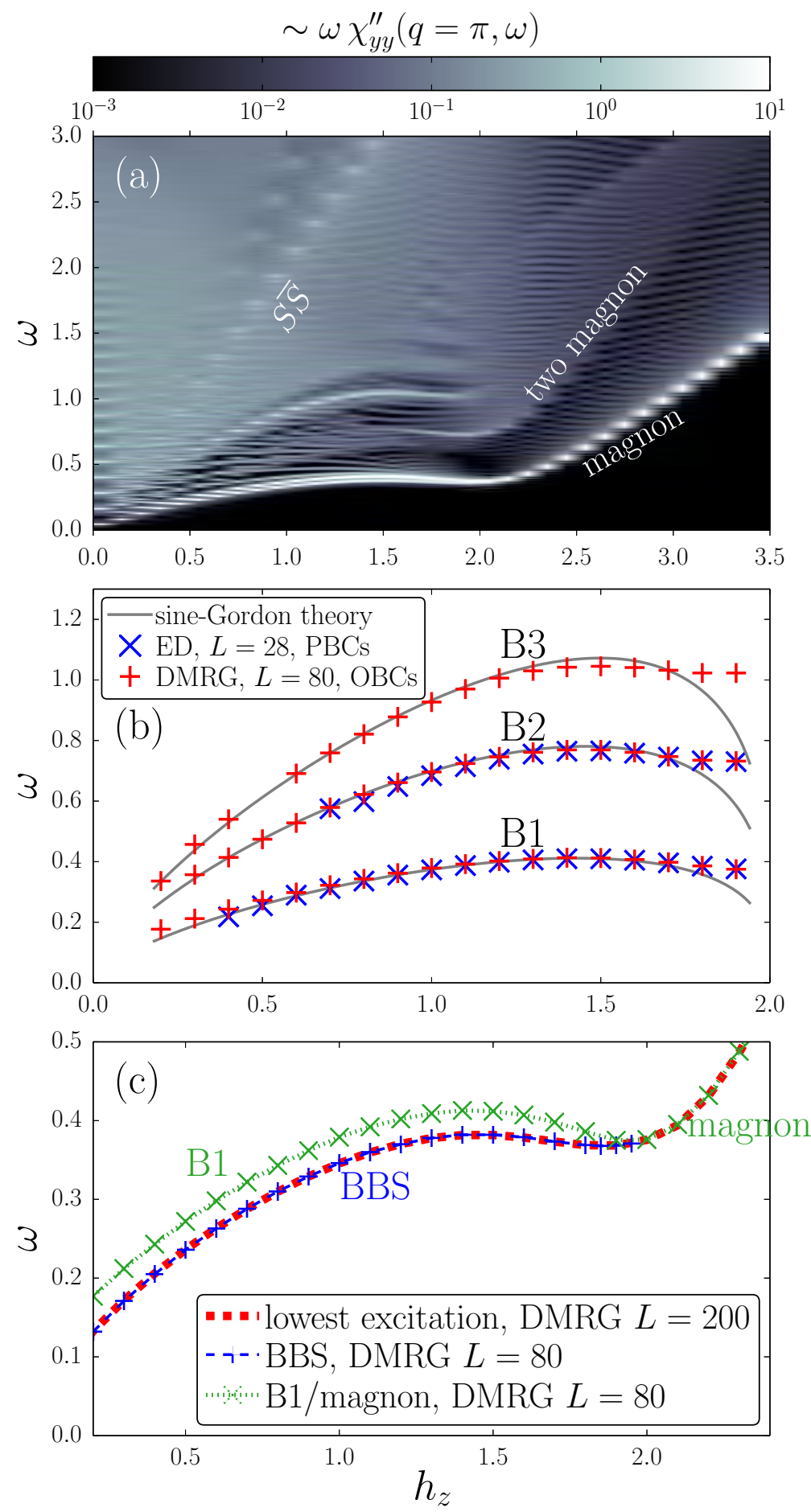

Figure 6.3: (Published in Ref. [THP $\left.{ }^{+} 16\right]$.) Frequency-field diagrams in the presence of an applied external field $h_{z}$ : (a) Frequency-field plot at $T=0$ of the absorption intensity $\omega \chi_{y y}^{\prime \prime}(q=\pi, \omega)$ obtained by DMRG-based Chebyshev expansions (order $N=6000$ ) at fixed fields $h_{z} \in[0,3.4]$ for a step increment of $\Delta h_{z}=0.1$ and $L=80$. (b) The frequencyfield dependence of the first (B1), second (B2), and third (B3) breather excitations obtained by DMRG for $L=80$ is compared to field theoretical and ED results. (c) DMRG calculations for the frequency-field dependence of the BBS and the first breather showing the transition to magnon physics towards high fields $(J=1$ and $c=0.083$ for all panels). The DMRG results for $L=200$ are taken from Ref. [ $\left.\mathrm{ZCO}^{+} 11\right]$. 


\section{\begin{tabular}{l|l} 
Single-soliton resonance & 6.5 .2
\end{tabular}}

The single-soliton resonance probed in ESR experiments using the Faraday configuration mainly originates from the uniform component of the transverse susceptibility $\chi_{+-}^{\prime \prime}(q=0, \omega)$ since its presence in the longitudinal staggered susceptibility $\chi_{z z}^{\prime \prime}(q=\pi, \omega)$ is suppressed in Eq. (6.3). However, since the intensity of the excitation is higher in $\chi_{z z}^{\prime \prime}(q=\pi, \omega)$ by about two orders of magnitude (cf. Fig. 6.5(c) below), the focus is on this component for the DMRG calculations. Figure 6.4 shows results for different system sizes $L$ and various values of the magnetic field $h_{z}$. In Fig. 6.4(a) the peak corresponding to the single-soliton resonance is the dominating feature at $h_{z}=1$. In addition, one observes the lower edge of a two-particle continuum at higher frequency. The extension of this continuum will be discussed in more detail in Sec. 6.5.4 where results for the momentumresolved dynamical spin structure factor are presented. The $L \rightarrow \infty$ extrapolation of the single-soliton resonance is represented by the dashed vertical line in Fig. 6.4(a) and is found below the field theoretical prediction $E_{S}=\sqrt{M_{S}^{2}+h_{z}^{2}}$ (solid vertical line). This discrepancy even persists for the smaller fields $h_{z}=0.25$ and 0.5 in Figs. 6.4(b)-(c), which focus on the region around the soliton resonance. By plotting the field dependence of the ratio $\omega / h_{z}$ for the soliton resonance in Fig. 6.5(b), this discrepancy is also confirmed by ED results for $L=28$ and PBCs which are in good agreement with the DMRG calculations. Thus, it cannot be a boundary effect. A very similar deviation from the same theory has been observed in ESR experiments on Cu-PM [ZKKF04] and will be discussed in detail in the next subsection.
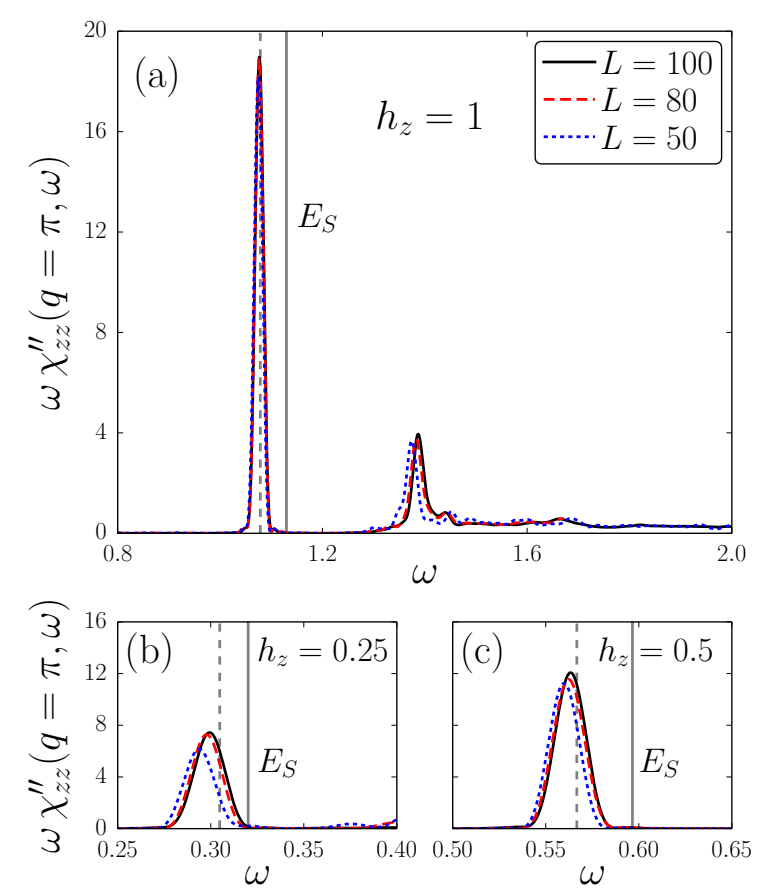

Figure 6.4: (Published in Ref. $\left.\left[\mathrm{THP}^{+} 16\right].\right)$ Zero-temperature DMRG results for the longitudinal uniform contribution to the ESR intensity for different system sizes $L$ and three values of the applied field $h_{z}$ : (a) $h_{z}=1$, (b) $h_{z}=0.25$, and (c) $h_{z}=0.5$. Here $J=1$ and $c=0.083$ are adopted in all panels. The dominant peak corresponds to the single-soliton resonance and is found below the field theoretical prediction $E_{S}=\sqrt{M_{S}^{2}+h_{z}^{2}}$ (solid vertical line). The dashed vertical line marks the result of the finite-size scaling. 

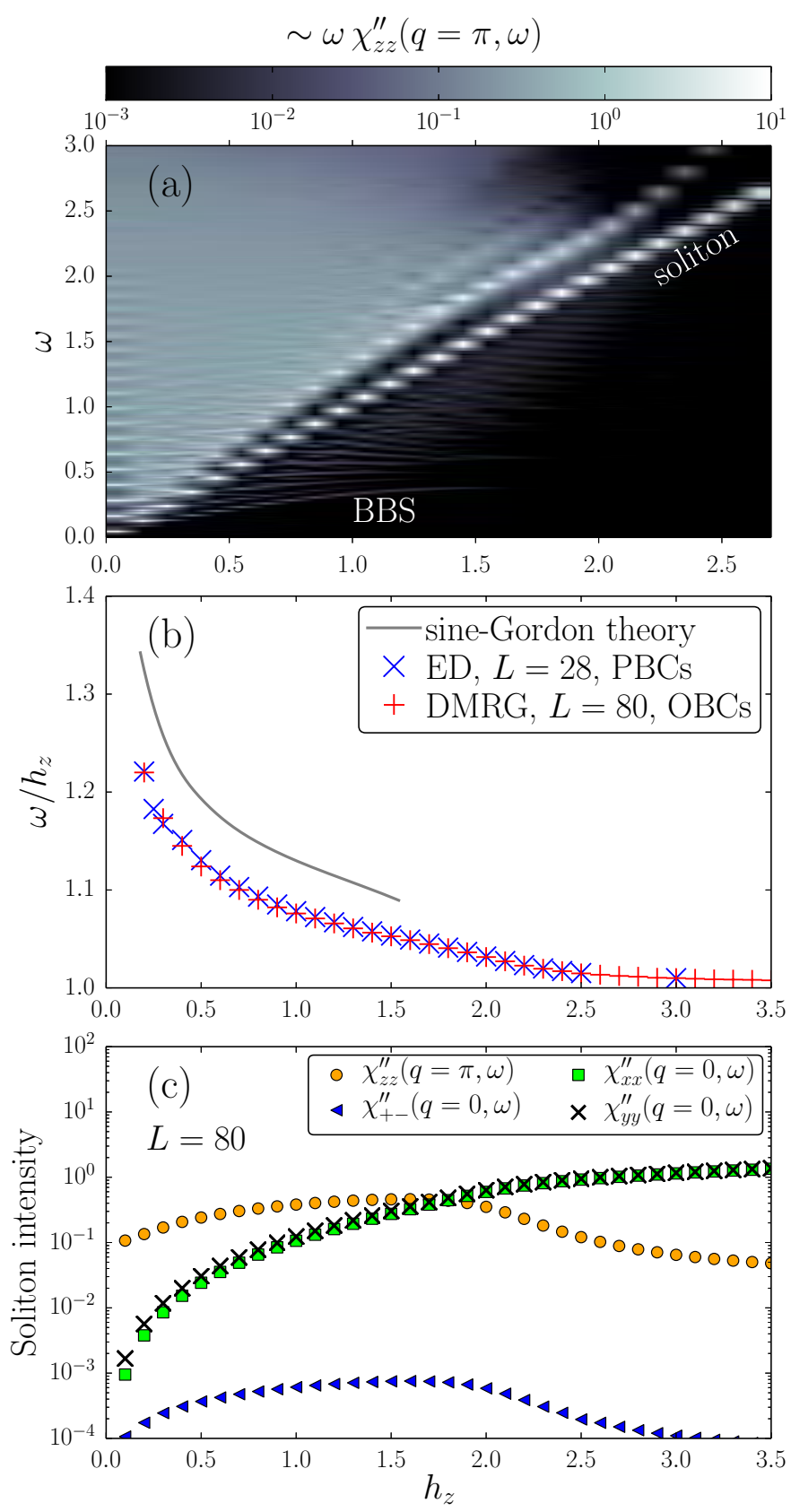

Figure 6.5: (Published in Ref. $\left[\mathrm{THP}^{+} 16\right]$.) Results for the soliton resonance $(J=1, c=0.083)$ : (a) Frequency-field plot of the intensity $\sim \omega \chi_{z z}^{\prime \prime}(q=\pi, \omega)$ obtained by DMRG-based Chebyshev expansions (order $N=4500$ ) at fixed fields $h_{z} \in[0,2.6]$ for a step increment of $\Delta h_{z}=0.1$ and $L=80$. Here $m=150$ is used corresponding to $\epsilon_{\text {compr }} \sim 10^{-4}$ at small fields. (b) Comparison of the ratio $\omega / h_{z}$ for the soliton as a function of the applied field between DMRG, $\mathrm{ED}$, and the sine-Gordon theory. Both numerical approaches find the soliton resonance below the field theory irrespective of the boundary conditions. (c) DMRG results for the integrated peak intensity of the soliton in different components of the dynamical susceptibility.

Next, the fate of the soliton excitation after the transition into the fully spin-polarized phase is discussed. To this end, the position and intensity of the single-soliton resonance is determined from the ESR absorption $\sim \omega \chi_{z z}^{\prime \prime}(q=\pi, \omega)$ obtained by DMRG calculations for different fields in Fig. 6.5(a) for a system of $L=80$ sites. The fact that the ratio $\omega / h_{z}$ approaches a value close to one for very high fields in Fig. 6.5(b) suggests that this excitation becomes the paramagnetic line which is located at $\omega=h_{z}$ in standard ESR experiments and perturbed by the small staggered field here. In Fig. 6.5(c), the integrated peak intensity from different components of the dynamical susceptibility is depicted as a function of the magnetic field. From this, one finds that the highest soliton intensity appears in the longitudinal staggered susceptibility $\chi_{z z}^{\prime \prime}(q=\pi, \omega)$, whereas the intensity of the paramagnetic line at high fields is largest in the uniform transverse susceptibilities $\chi_{x x}^{\prime \prime}(q=0, \omega)$ and $\chi_{y y}^{\prime \prime}(q=0, \omega)$. An additional frequency-field diagram for the intensity $\sim \omega \chi_{x x}^{\prime \prime}(q=0, \omega)$ is provided in Fig. A.1(b) in the appendix. 


\section{\begin{tabular}{l|l} 
Comparison to the experiment & 6.5 .3
\end{tabular}}

In the following, the DMRG results are related to the experimentally determined ESR resonance modes in the material $\mathrm{Cu}-\mathrm{PM}$ from Ref. [ZKKF04]. Since the only free parameter $c$ has been determined as $c=0.08$ by a fit to the B1 mode in this previous experiment [ZKKF04], the value is adopted in the calculations for the comparison to the experiment. First, the frequency-field dependence of the breather excitations is compared in Fig. 6.6. The resonance modes B1 and B3 are extracted from the absorption $\sim \omega \chi_{y y}^{\prime \prime}(q=\pi, \omega)$ for $L=80$ as their intensity is higher in this component. The B2 mode is again determined from the intensity $\sim \omega \chi_{+-}^{\prime \prime}(q=\pi, \omega)$. Apart from the finite-size effects towards small magnetic fields (discussed earlier in Figs. 6.2(b)-(c)), there is good agreement between DMRG and experiment.

Next, the experimental data for the frequency-field dependence of the single-soliton resonance in $\mathrm{Cu}-\mathrm{PM}$ is compared to sine-Gordon and DMRG results for the intensity $\omega \chi_{z z}^{\prime \prime}(q=\pi, \omega)$. Figure 6.7 shows this comparison for two values of the material parameter $c$ from the literature, $c=0.08$ and $c=0.083$ [ZKKF04, ZKKF05]. One of the main results is that there is very good agreement between the DMRG results and the experiment. Moreover, these results are both found below the sine-Gordon field theory irrespective of the $c$-value. The reason for the deviation can be understood by considering the momentum dependence of the dynamical structure factor $S_{z z}(q, \omega)=\chi_{z z}^{\prime \prime}(q, \omega) / \pi$ in Fig. 6.8(c). For $q \sim \pi$, the dominating feature is the dispersion relation of the antisoliton, which clearly exhibits curvature. This curvature comes from irrelevant operators which break Lorentz invariance [OA02]. Therefore, the Lorentz invariant dispersion $E_{S}=\sqrt{M_{S}^{2}+h_{z}^{2}}$ used by the field theory is not perfectly suitable for describing the single-soliton resonances appearing at $q=\pi$ in $\chi_{z z}^{\prime \prime}(q, \omega)$ and at $q=0$ in $\chi_{+-}^{\prime \prime}(q, \omega)$.

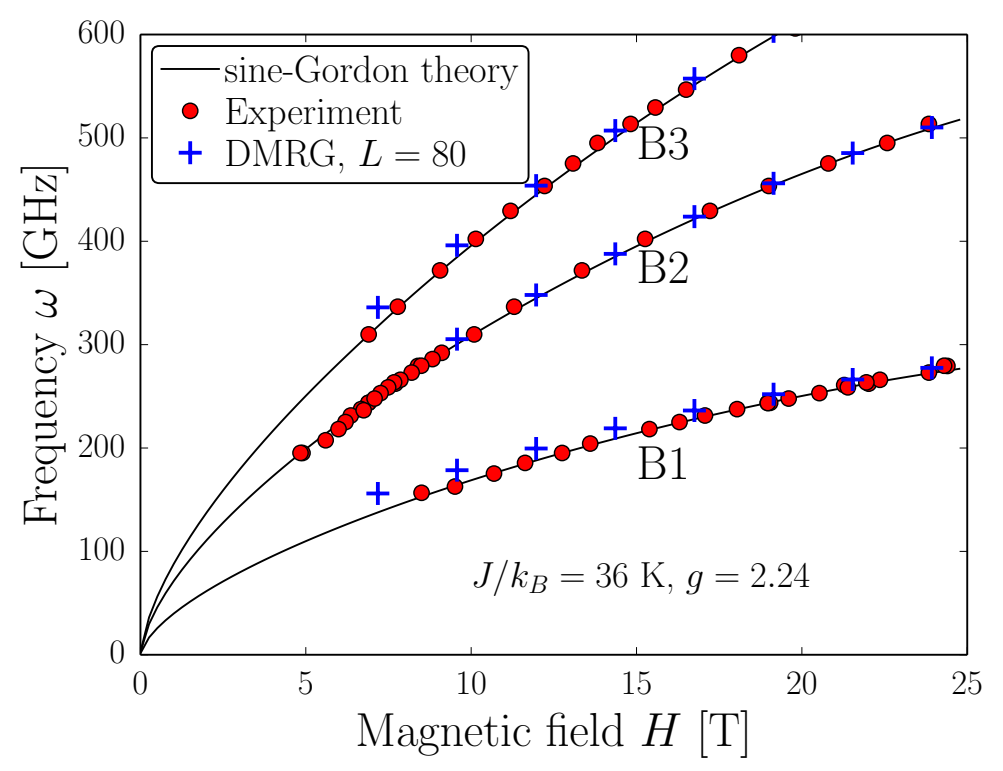

Figure 6.6: (Published in Ref. [THP $\left.{ }^{+} 16\right]$.) Comparison between DMRG $(L=80)$, experimental, and field theoretical results for the frequency-field dependence of the breather excitations $(c=0.08)$. The experimental data are taken from Ref. [ZKKF04]. 


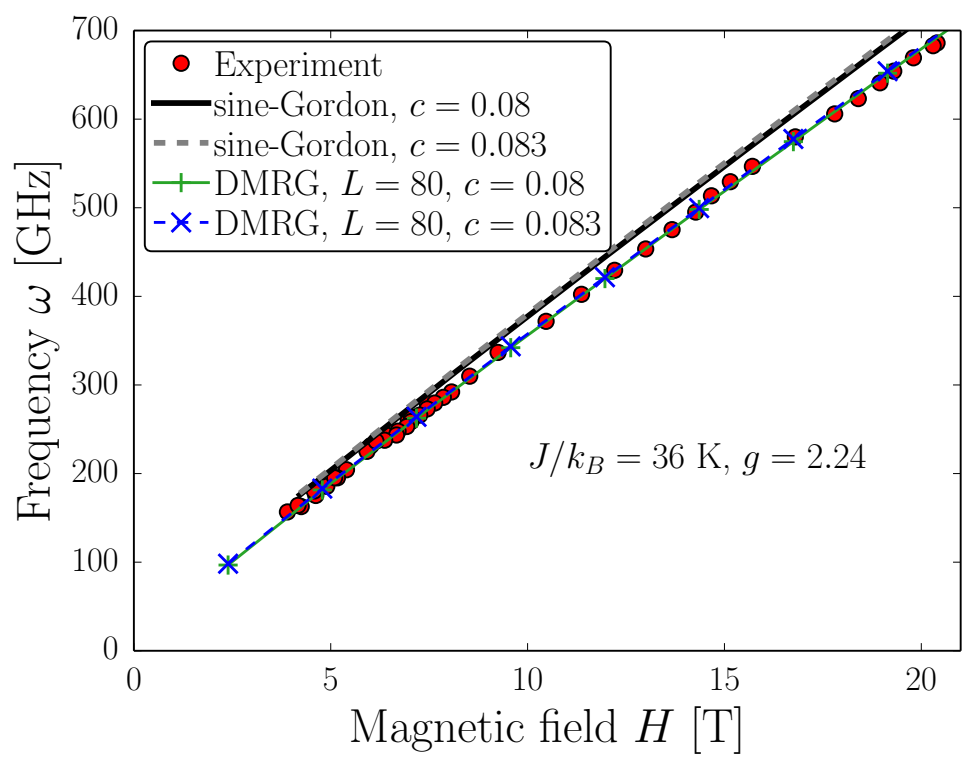

Figure 6.7: (Published in Ref. [THP $\left.{ }^{+} 16\right]$.) Comparison between DMRG $(L=80)$, experimental, and field theoretical results for the frequency-field dependence of the single-soliton resonance. The experimental data are taken from Ref. [ZKKF04].

Moreover, it is important to also discuss the presence of boundary modes in $\mathrm{Cu}-\mathrm{PM}$. Due to the very low impurity concentration in the sample, the BBS just below B1 is not experimentally observed [ZKKF04, $\left.\mathrm{ZCO}^{+} 11\right]$. Since the DMRG calculations are performed with open boundaries, this BBS has been clearly identified in Figs. 6.2 and 6.3(c). According to the boundary field theory [FO12], there should be even more boundary resonances at $T=0$. However, none of them is clearly observable in the DMRG results for the spectral functions since their intensity is too low. Ref. [FO12] argues that some of these additional modes can be assigned to the unknown modes, which were observed in $\mathrm{Cu}-$ $\mathrm{PM}\left[\mathrm{ZKKF} 04, \mathrm{ZCO}^{+} 11\right]$ and to similar modes observed in $\mathrm{KCuGaF}_{6}$ [ $\left.\mathrm{UTO}^{+} 09\right]$. These unexplained resonances could previously not be accounted for in the bulk sine-Gordon theory. However, the fact that the BBS was not observed in $\mathrm{Cu}-\mathrm{PM}$, while a significant BBS weight is found in the numerical calculations for finite chains, indicates the Cu-PM samples to be very clean. Now the other boundary modes seem to have so low spectral weight that they are unobservable even in the numerical computations. Thus, one concludes that these additional boundary modes are unlikely to explain the unexplained experimental $\mathrm{U} 1$ and $\mathrm{U} 2$ modes in $\mathrm{Cu}-\mathrm{PM}$.

\section{\begin{tabular}{l|l} 
Dynamical spin structure factor & 6.5 .4
\end{tabular}}

Up to this point, the results are obtained for the momenta $q=0$ and $q=\pi$, which are relevant for a comparison to ESR experiments (cf. Eq. (6.3)). Now the full momentum dependence of the elementary excitations at $T=0$ will be investigated for a magnetic field of magnitude $h_{z}=1$. Figure 6.8 shows DMRG results for the $x x, y y$, and $z z$ 
components of the dynamical spin structure factor $S_{\alpha \alpha}(q, \omega)$ for an $L=80$ site chain. For momentum-resolved quantities, the spin operators in $q$ space can be defined as [BGJ04]

$$
S_{q}^{\alpha}=\sqrt{\frac{2}{L+1}} \sum_{i=1}^{L} \sin (q i) S_{i}^{\alpha}
$$

with respect to the quasi-momenta $q=\pi n /(L+1)$ and integers $n=1, \ldots, L$. The transverse components of the dynamical structure factor in Figs. 6.8(a)-(b) contain the soliton dispersion which assumes a minimum at the incommensurate wavevector $q=q_{0}$. The minimum of the antisoliton dispersion at $q=\pi-q_{0}$ is a main feature of the $z z$ component shown in Fig. 6.8(c). In the yy $(z z)$ component, the soliton continuously merges into the B1 dispersion, which has its minimum at the antiferromagnetic wavevector $q=\pi$ (resp. $q=0$ ). Furthermore, one can identify the heavier breathers B2 and B3 at $q=\pi$ in $S_{x x}$ and $S_{y y}$. Interestingly, there is a manifestation of the BBS in all three components of the dynamical structure factor, while the most intense signal occurs in the yy component in Fig. 6.8(b). As expected for a localized mode, one also finds that the BBS has a flat dispersion [FO12].
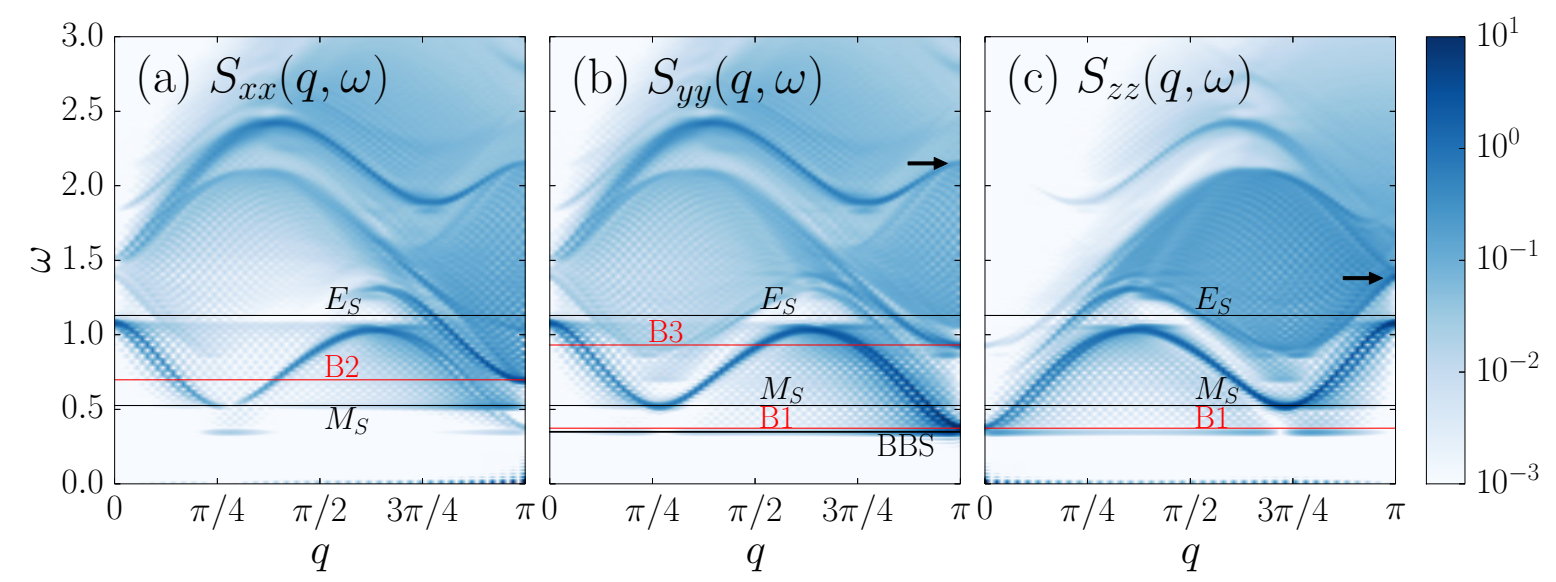

Figure 6.8: (Published in Ref. $\left[\mathrm{THP}^{+} 16\right]$.) DMRG results for the $x x, y y$, and $z z$ components of the dynamical spin structure factor at $T=0$ for $h_{z}=1$ and $c=0.083$ using a uniform broadening of $\eta=0.012\left(J=1, L=80, m=150\right.$ and $\left.\epsilon_{\mathrm{compr}} \sim 10^{-6}\right)$. The horizontal solid lines represent the frequencies of the elementary excitations from the sine-Gordon theory. Most importantly, the curvature of the soliton dispersion for $q \sim 0$ in panel (a) resp. the curvature of the antisoliton for $q \sim \pi$ in panel (c) lead to a deviation from the Lorentz invariant dispersion. The arrows at $q=\pi$ in panels (b) and (c) mark the soliton-antisoliton excitation $S \bar{S}$ and the lower edge of the continuum, respectively.

The results in Fig. 6.8 represent an improvement over a previous ED investigation of the dynamical structure factor for $L \sim 20$ in Ref. $\left[\mathrm{KBC}^{+} 05\right]$. These ED calculations for $c=0.075$ are in agreement with neutron scattering results for the low-energy modes in dimethylsulfoxide $\mathrm{CuCl}_{2}$ published in the same work. The DMRG calculations for $L=80$ in Fig. 6.8 provide a higher momentum and frequency resolution. Thus, the multi-particle continua can be resolved more clearly. As a main result, the curvature of the soliton dispersion is observed for $q \sim 0$ in Fig. 6.8(a) resp. the curvature of the antisoliton for $q \sim \pi$ in Fig.6.8(c). The presence of this curvature is also implied by previous ESR experiments [ZKKF04] since the single-soliton resonance is found below its field theoretical prediction $E_{S}=\sqrt{M_{S}^{2}+h_{z}^{2}}$ (see Sec. 6.5.3). 
Moreover, multi-particle continua are observed at higher frequency. In particular, the extended continuum in Fig.6.8(c) was analyzed by using the following consideration. Since there is a continuous one-particle dispersion $\epsilon_{1}(q)$ throughout the Brillouin zone, it is possible to construct the energies of the two-particle excitations at $q_{1}+q_{2}$ by $\epsilon_{2}\left(q_{1}+q_{2}\right)=\epsilon_{1}\left(q_{1}\right)+\epsilon_{1}\left(q_{2}\right)$. Indeed, it is found that the boundaries of the continuum mostly coincidence with the extremal $\epsilon_{2}\left(q_{1}+q_{2}\right)$. Thus, the continuum corresponds to the continuous dispersion linking the first breather and the soliton.

An important resonance labeled as ' $S \bar{S}$ ' in the DMRG calculations for the absorption intensity $\sim \omega \chi_{y y}^{\prime \prime}(q=\pi, \omega)$ in Fig. 6.3(a) is also found in the yy component of the dynamical spin structure factor (see Fig.6.8(b)) where it is marked by an arrow. This feature is found at an energy of twice the single-soliton resonance and therefore consistent with a soliton-antisoliton excitation. It represents the singularity at the upper edge of a continuum.

\section{\begin{tabular}{l|l} 
Finite-temperature results & 6.6
\end{tabular}}

Motivated by experimental hints of strong temperature dependencies of ESR line widths in $\mathrm{Cu}-\mathrm{PM}$ [Zvy12], the temperature effects on the ESR intensity of this material is studied. The focus is on the wavevectors $q=0$ and $q=\pi$, which are relevant for a comparison to ESR experiments, cf. Eq. (6.3). With increasing temperature, the redistribution of spectral weight and, in particular, the emergence of thermally-induced transitions between zero-temperature excitations of the sine-Gordon theory are investigated. For the breather and interbreather excitations, there are both experimental and numerical results in Sec. 6.6.1. In the numerical results, the observation of a soliton-breather transition is discussed in Sec.6.6.2. Moreover, temperature effects may also lead to a crossover between excitations. As an example, the temperature dependence of the soliton is studied in Sec. 6.6.3.

\section{\begin{tabular}{l|l} 
Breather and interbreather excitations & 6.6 .1
\end{tabular}}

\section{Numerical results}

First of all, the focus is set on the temperature dependence of the breathers at $h_{z}=1$. To this end, both contributions, $I_{x x}(q=\pi, \omega)$ and $I_{y y}(q=\pi, \omega)$, to the staggered transverse ESR intensity for $L=50$ are studied in Fig. 6.9. It is important to note that B2 is contained in the former component and B1 as well as B3 in the latter. These finitetemperature DMRG calculations for OBCs are obtained by a Chebyshev expansion with respect to the Liouville operator. In contrast to the purely $T=0$ results shown at a broadening of $\eta=0.01$ in Fig. 6.2(a), the resolution in Fig. 6.9 assumes the value $\eta=0.035$ at $T>0$ and is therefore not as high as at $T=0$. This is due to the increased computational effort for purifying the thermal density matrix as well as applying the Liouville operator, whose spectral width is twice the width of the Hamiltonian and, most importantly, directly proportional to the broadening. As a consequence of this limitation, the BBS and B1 are not resolved as two separate peaks at $T=0$ in Fig. 6.9. However, the obtained resolution is high enough to see that at higher temperature the breather 
excitations are clearly subject to thermal broadening. In particular, B3 is only observable at $T=0$ in the DMRG computations due to this effect.

Since thermally activated interbreather transitions at frequencies $M_{n}-M_{m}(n>m)$ have been reported in the sine-Gordon magnet $\mathrm{KCuGaF}_{6}\left[\mathrm{UTO}^{+} 09\right]$, it is interesting to look for these excitations in the numerical calculations for $\mathrm{Cu}-\mathrm{PM}$. Therefore, the resolution of the DMRG calculation at $T / J=0.5$ is enhanced in the inset of Fig. 6.9(b), which focuses on the region around the field theoretical value for $M_{3}-M_{2}$ (solid vertical line). Very close to this frequency a weak maximum is found which can be interpreted as evidence for the $M_{3}-M_{2}$ interbreather transition. Unfortunately, it is not possible to observe further excitations of this type in the DMRG calculations. For instance, the observation of a possible feature at $\omega=M_{2}-M_{1}$ is obstructed by the choice of OBCs. More precisely, the large BBS intensity appears very close to this frequency. Therefore, ED calculations with PBCs are a viable possibility for the detection of further interbreather transitions, since the BBS is absent in this case. The ED results are shown for $L=20$ at $T=0.5$ resp. $L=24$ at $T \leq 0.25$ in Fig. 6.10. Here the magnetic field is chosen as $h_{z}=0.6$, which corresponds to a field of about $14.36 \mathrm{~T}$ in experiments on $\mathrm{Cu}-\mathrm{PM}$ discussed further below. The vertical solid lines in the insets of Figs. 6.10(a)-(b) highlight the frequencies $M_{n}-M_{m}$ at which the excitations are expected. However, here these predictions are not determined via the sine-Gordon theory but from the finite-size positions of the breathers, as B2 and B3 are still subject to finite-size effects for $L=24$. In both the $x x$ and the yy component of the ESR intensity, the transitions at $M_{2}-M_{1}$ and $M_{3}-M_{1}$ are clearly visible and match the expected frequencies. Fig. 6.10(c) shows the spectral weight of the

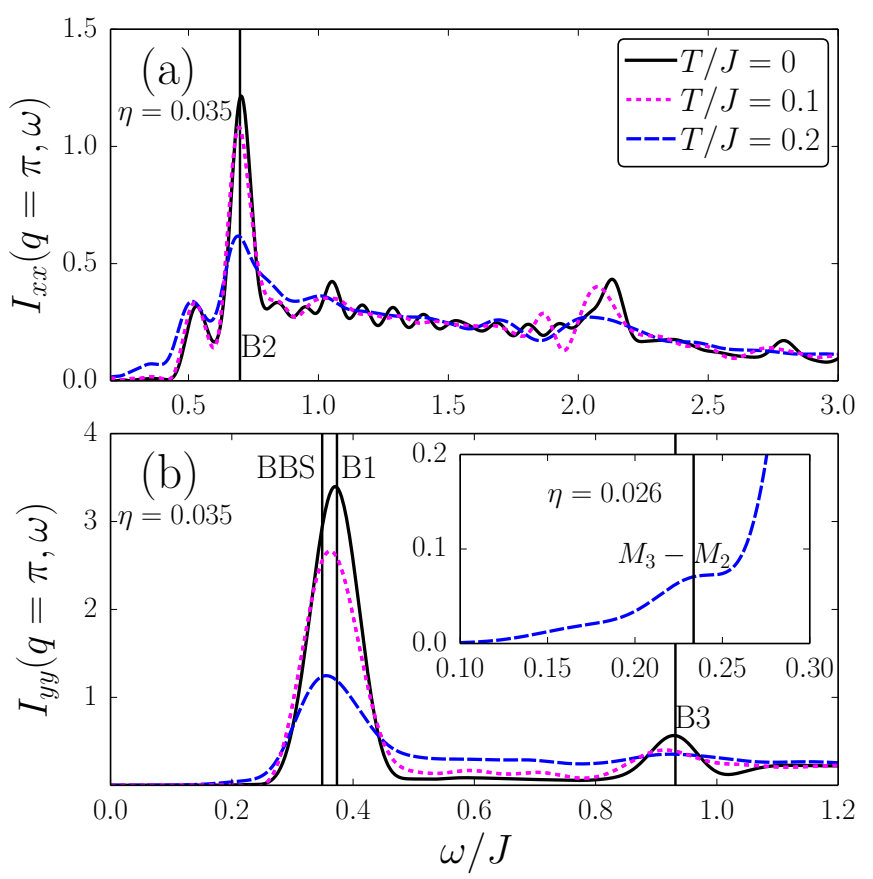

Figure 6.9: (Published in Ref. $\left[\mathrm{THP}^{+} 16\right]$.) DMRG results for the temperature dependence of breather excitations in the transverse ESR intensities $I_{x x}(q=\pi, \omega)$ (a) and $I_{y y}(q=\pi, \omega)$ (b) for a magnetic field of $h_{z}=1$ and $c=0.083$. The solid vertical lines mark the $T=0$ sine-Gordon predictions. Inset: DMRG calculations with enhanced resolution provide evidence for the interbreather transition at $M_{3}-M_{2}$ (solid vertical line). 
B1-B2 transition as a function of temperature for $L=24$. Here, the coefficient of the corresponding delta-function in the spectral representation Eq. (2.29) has been computed, i.e., the thermal occupation of the B1 mode multiplied with the matrix elements for the transition to B2. The effect is that the temperature dependence of all three quantities in Fig. 6.10(c) is identical, just the matrix elements are different. It is observed that the B1-B2 transition is thermally activated at low $T$, goes through a maximum a little below $T / J=0.15$, and then decays again towards high temperature. The latter is also reflected in panels (a) and (b) of Fig. 6.10 where one observes that upon increasing the temperature to $T / J=0.5$, these weak-intensity features become hardly distinguishable from the finite-temperature background. Thus, the appearance of these transitions, which is expected from the sine-Gordon theory [FO12], is limited to low temperatures only.
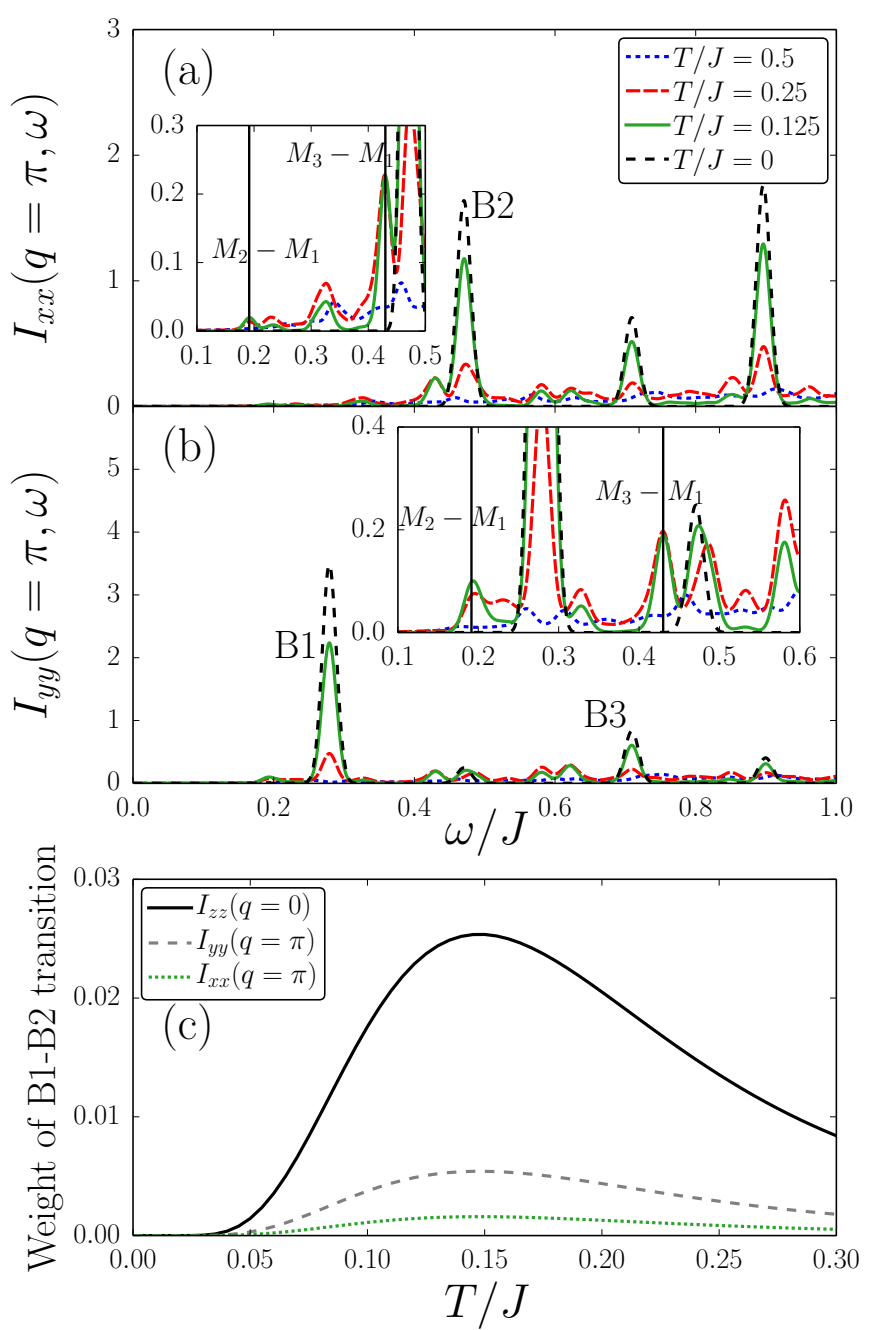

Figure 6.10: (Published in Ref. $\left[\mathrm{THP}^{+} 16\right]$.) ED results for systems with PBCs addressing the temperature dependence of breather and interbreather excitations at a magnetic field of $h_{z}=0.6$ and $c=0.08$. The transverse ESR intensities $I_{x x}(q=\pi, \omega)$ resp. $I_{y y}(q=\pi, \omega)$ are shown for $\eta=0.01$ in panels (a) and (b). Here results for $L=24$ at $T \leq 0.25$ and $L=20$ at $T=0.5$ are shown. The insets focus on finite-temperature transitions at $M_{n}-M_{m}$ between breather excitations, which are marked by solid vertical lines. (c) Temperature-dependence of the spectral weight of the interbreather transition between B1 and B2 calculated from different components of the absorption intensity in dependence of temperature $(L=24)$. 


\section{Experimental results}

High-field ESR experiments on Cu-PM were performed using a 16 T superconducting magnet ESR spectrometer (similar to that described in Ref. [ZKvL $\left.{ }^{+} 04\right]$ ), equipped with VDI sources of millimeter-wave radiation (product of Virginia Diodes Inc.) and a transmissiontype probe in the Faraday configuration. The field sweep rate was $0.5 \mathrm{~T} / \mathrm{min}$. The magnetic field was applied along the $c^{\prime \prime}$ direction, which is characterized by the maximal value of the staggered magnetization for $\mathrm{Cu}-\mathrm{PM}\left[\mathrm{FAG}^{+} 00\right]$. High-quality single-crystals of $\mathrm{Cu}$ PM with typical size of $3 \times 3 \times 1 \mathrm{~mm}^{3}$ were used that have been grown by slow evaporation of the equimolar aqueous solution of copper nitrate and pyrimidine [INN $\left.{ }^{+} 97, \mathrm{YIA}^{+} 01\right]$.

Figure 6.11(a) shows the temperature dependence of ESR absorption spectra in Cu-PM measured at $178 \mathrm{GHz}$. The most prominent feature is the first breather B1 which is clearly visible up to temperatures $T \sim 3 \mathrm{~K}$. Furthermore, the measurement confirms that there is no evidence for the presence of the BBS towards higher fields. The only stable feature observed in addition to B1 is identified as the interbreather transition at $M_{2}-M_{1}$. The inset of Fig. 6.11(a) shows the corresponding absorption minimum at $T=2.2 \mathrm{~K}$ for a few selected frequencies. By measuring the frequency-field diagram over a broad range of the applied magnetic field, it is shown that there is excellent agreement with sineGordon and DMRG results for $M_{2}-M_{1}$ in Fig. 6.11(b). In the DMRG calculations, $M_{1}$ and $M_{2}$ were determined as the peak position of B1 and B2 in the absorption intensities $\sim \omega \chi_{y y}^{\prime \prime}(q=\pi, \omega)$ resp. $\sim \omega \chi_{x x}^{\prime \prime}(q=\pi, \omega)$ at $T=0$ for large systems of $L=120$ to minimize finite-size effects at small magnetic fields.
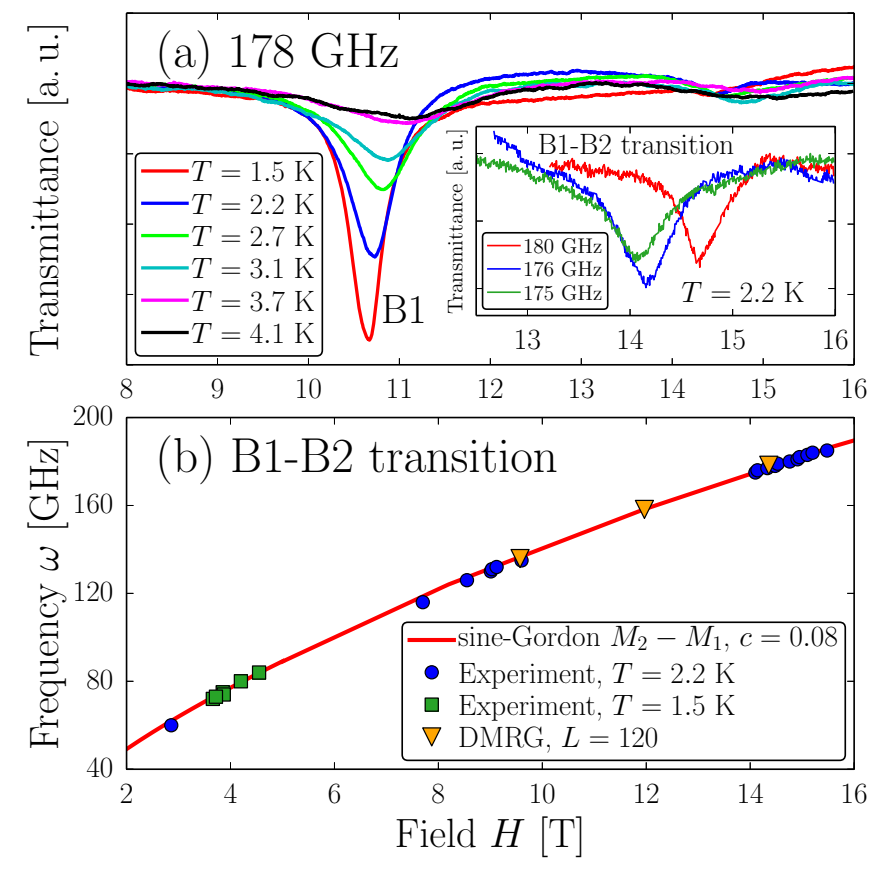

Figure 6.11: (Published in Ref. $\left[\mathrm{THP}^{+} 16\right]$.) (a) Temperature dependent ESR absorption intensity of the first breather B1 at $178 \mathrm{GHz}$. There is no signature of the BBS towards higher fields, but at about $H=14.5 \mathrm{~T}$ the interbreather transition between $\mathrm{B} 1$ and $\mathrm{B} 2$ is observed. The inset shows this excitation for different frequencies at $T=2.2 \mathrm{~K}$. (b) Frequency-field plot of the B1-B2 interbreather transition comparing the ESR modes with $M_{2}-M_{1}$ from the sine-Gordon theory and zero-temperature DMRG calculations for $L=120$. 
The signals of the interbreather transition in the present ESR experiments are rather weak at a temperature of $T / J \approx 0.06$. Therefore, it is surprising that the authors of Ref. [UTO $\left.{ }^{+} 09\right]$ report that the intensities of breather and interbreather excitations are of the same order in $\mathrm{KCuGaF}_{6}$ at an even much lower temperature $(T / J \approx 0.005)$. However, it has to be mentioned that their ESR measurements have been performed in a pulsed magnetic field using a larger sample of size $3 \times 3 \times 3 \mathrm{~mm}^{3}$. This is different from the ESR experiments shown in the present chapter as they were obtained in a static magnetic field.

\section{Soliton-breather transition}

Based on field theory, soliton-breather transitions at frequencies $\left|E_{S}-M_{n}\right|$ have been predicted to occur at finite temperature [FO12]. However, nothing has been known about the corresponding intensities so far. The ED results for $L=24$ resolve the low-intensity transition between the soliton and the first breather at $\omega=E_{S}-M_{1}$ in the intensity $I_{z z}(q=\pi, \omega)$. The temperature dependence of this feature is shown in the insets of Fig. 6.12 for magnetic fields of $h_{z}=0.6$ and $h_{z}=1$. The transition assumes its maximum intensity around a temperature of $T / J=0.25$, while it is hardly visible at $T / J=0.5$. Therefore, this feature occurs in the same temperature range as the interbreather transitions.
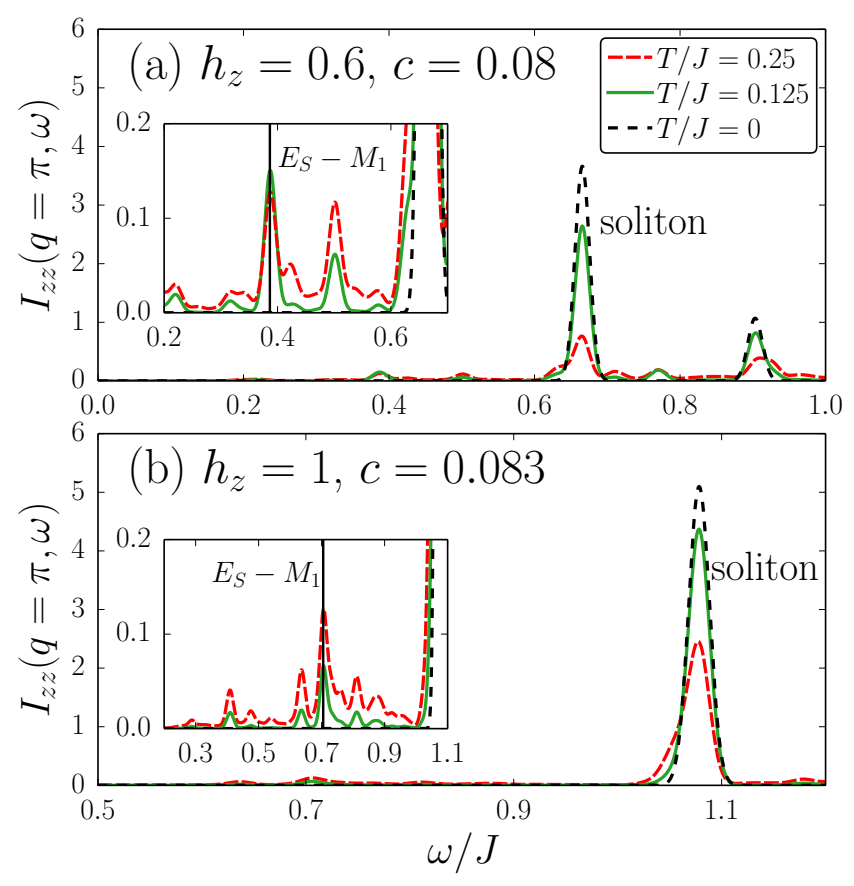

Figure 6.12: (Published in Ref. [THP $\left.{ }^{+} 16\right]$.) ED results for $L=24, \eta=0.01$, and PBCs addressing the temperature dependence of the soliton and the transition between the soliton and the first breather for different values of the magnetic field $h_{z}=0.6$ (a) and $h_{z}=1$ (b). The finite-temperature transition $E_{S}-M_{1}$ is highlighted by the solid vertical lines in the two insets. 


\section{Crossover: soliton to paramagnetic line $\mid 6.6 .3$}

Now, the temperature-induced crossover between the soliton at low temperature and the paramagnetic line perturbed by the staggered field at higher temperature is studied. For small systems, Iitaka and Ebisuzaki have presented results [IE03] using their Boltzmannweighted time-dependent method which is based on a random vector representation for the evaluation of the trace and a Chebyshev expansion of the Boltzmann operator. Results for lattices of $L=16$ sites have been published. The finite-temperature DMRG approach developed in this thesis is used to revisit this feature by providing state-of-the-art results for larger systems with $L=50$ sites. On this basis, the interpretation given in Ref. [IE03] is corrected. The DMRG results for different temperatures at $h_{z}=1$ are shown in Fig. 6.13(a). Figure 6.13(b) contains ED results at $h_{z}=1$. Here systems with $L=24$ $(T / J \leq 0.25)$ and $L=20$ sites at $T / J=0.5$ are considered. In Appendix A.2 a detailed finite-size analysis for these data is shown. ED results for $h_{z}=0.6$ are depicted in Fig. 6.13(c). For both magnitudes of the magnetic field, it is intriguing how quickly an increase in temperature redistributes spectral weight from the soliton at $T=0$ to the paramagnetic line perturbed by the staggered field at $\omega \approx h_{z}$ and that this crossover can be traced numerically.

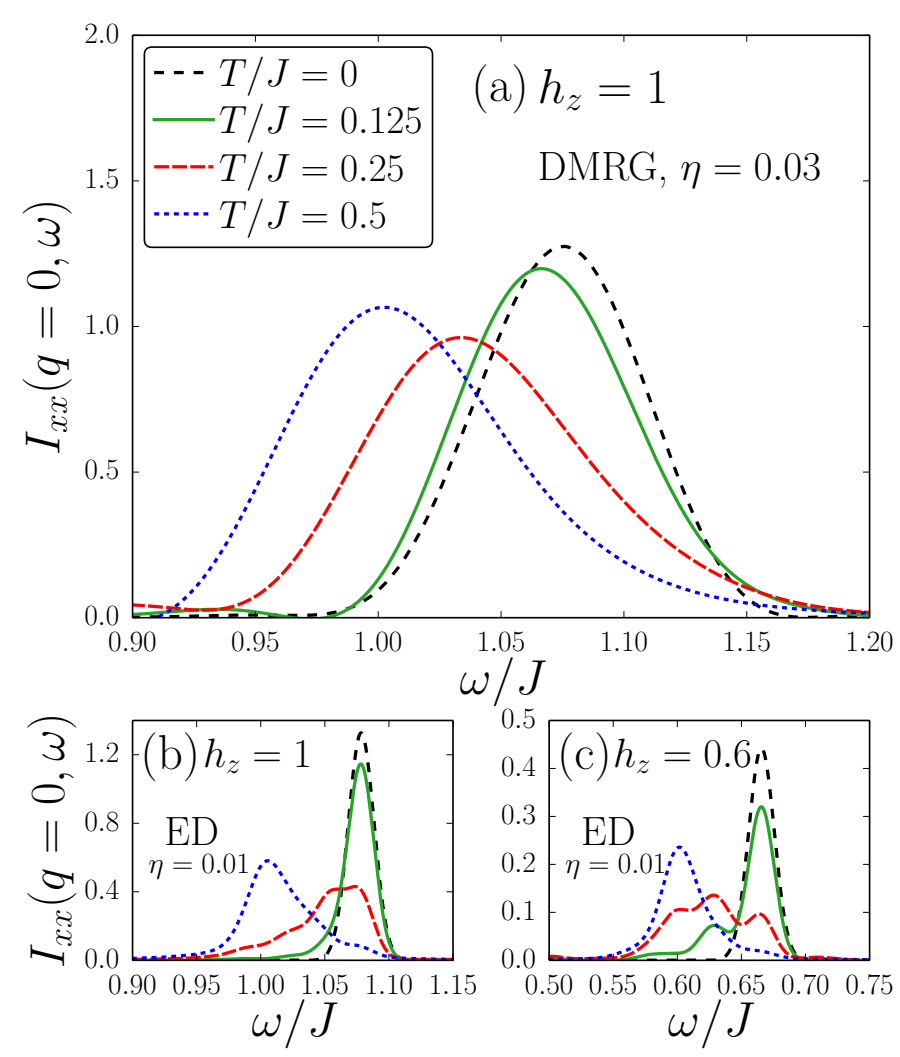

Figure 6.13: (Published in Ref. $\left[\mathrm{THP}^{+} 16\right]$.) Temperature-induced crossover between the soliton at low temperature and the paramagnetic line at higher temperature in $I_{x x}(q=0, \omega)$ : (a) DMRG results for $L=50$ in the presence of a uniform magnetic field $h_{z}=1$ and $c=0.083$ with resolution $\eta=0.03$. (b) ED results for systems with PBCs at higher resolution $\eta=0.01$, $h_{z}=1$, and $c=0.083$. (c) ED results for systems PBCs at $h_{z}=0.6$ and $c=0.08$ with resolution $\eta=0.01$. The ED results are shown for $L=24(T / J \leq 0.25)$ and $L=20$ at $T / J=0.5$. 


\section{\begin{tabular}{l|l} 
Conclusion & 6.7
\end{tabular}}

Spectral functions for the material $\mathrm{Cu}-\mathrm{PM}$ have been computed with unprecedented accuracy using DMRG and ED. At $T=0$, the intensities and the frequency-field dependence of the breather excitations and the BBS found directly below the first breather, which was predicted by a boundary sine-Gordon field theory in Ref. [FO12], were studied. Adopting OBCs for the DMRG calculations, it was shown that the BBS intensity scales to zero in the thermodynamic limit. Moreover, the first breather and the BBS merge into one single excitation close to the saturation field. Besides the BBS, Furuya and Oshikawa also predicted additional boundary modes at $T=0$ and in the case of $\mathrm{Cu}-\mathrm{PM}$ assigned two of them to the previously unexplained modes $\mathrm{U} 1$ and $\mathrm{U} 2$ found for this compound [FO12]. These additional boundary resonances are not observable in the DMRG calculations for $\mathrm{Cu}-\mathrm{PM}$. Thus, one concludes that their intensities must be so low that they are unlikely to explain the U1 and U2 modes. This conclusion is supported by the fact that in ESR experiments not even the boundary mode with the highest intensity is observed [ZKKF04]. The absence of boundary effects in the experiments can be explained by the high purity of the $\mathrm{Cu}-\mathrm{PM}$ samples. A second conclusion in this context is that there are no signatures for the experimental modes U1, U2, or U3 [ZKKF04] in the DMRG computations, which suggests that these modes in $\mathrm{Cu}-\mathrm{PM}$ may not be contained in the effective model in Eq. (4.13). They might occur as a consequence of further effects beyond this effective model. Possible candidates are additional anisotropies or interchain coupling.

Another main finding is that the DMRG results provide a better description for the frequency-field dependence of the single-soliton resonance in the material $\mathrm{Cu}-\mathrm{PM}$ [ZKKF04] than the sine-Gordon theory. The reason is that the Lorentz invariant dispersion relation $E_{S}=\sqrt{M_{S}^{2}+h_{z}^{2}}$ used by the field theory does not capture the band curvature generated by the coupling of marginally irrelevant operators. This is supported by the DMRG calculations for the momentum and frequency-resolved dynamical spin structure factor in Fig. 6.8. Furthermore, recent inelastic neutron scattering experiments on $\mathrm{KCuGaF}_{6}$ $\left[\mathrm{UTK}^{+} 15\right]$ probed the dispersion branch along which the soliton and antisoliton are located at the incommensurate wavevectors $q_{s}=\pi \pm q_{0}$ as sketched in Fig. 4.3. It can be concluded that these experimental results are also compatible with the occurrence of band curvature and the single-soliton resonance at $q=\pi$.

At $T>0$, the temperature dependence of the breather and thermally activated interbreather transitions was investigated. In the ED calculations with PBCs contributed by Andreas Honecker, various interbreather excitations were observed up to temperatures of about $T / J=0.5$ at both $h_{z} / J=0.6$ and $h_{z} / J=1$. In Fig. 6.10(c) the transition at $M_{2}-M_{1}$ shows maximum intensity at about $T / J=0.15$ and quickly decays upon increasing the temperature. This interbreather excitation was also observed below the first breather over a wide field range in ESR experiments on Cu-PM. The frequency-field dependence is in excellent agreement with sine-Gordon theory. Unfortunately, the open boundaries for the DMRG computations are not convenient in this case since the high BBS intensity is located very close to the frequency of the strongest interbreather excitation $M_{2}-M_{1}$, which could therefore not be resolved. However, evidence for the $M_{3}-M_{2}$ transition is found in the DMRG data. 
Finally, the single-soliton resonance was revisited, which with increasing temperature crosses over to the paramagnetic line perturbed by the staggered field. This has been studied before by Iitaka and Ebisuzaki for small systems $(L=16)$ using their Boltzmannweighted time-dependent method [IE03]. The finite-temperature DMRG approach working directly in the frequency domain enables us to correct their interpretation by studying larger systems with $L=50$ sites. 


\section{Magnetic excitations in the spin- $1 / 2$ dimerized-chain compound $\mathrm{BaCu}_{2} \mathrm{~V}_{2} \mathrm{O}_{8}$}

The main content of the present chapter has been published as a Rapid Communication in Physical Review B $\left[\mathrm{KTF}^{+} 16\right]$. Some parts the following chapter resemble the manuscript and the supplemental material of Ref. [KTF $\left.{ }^{+} 16\right]$. The manuscript as well as the supplemental material contain major contributions from many authors including me. In addition, I performed the DMRG calculations. Together with Salvatore R. Manmana and Andreas Honecker, I also contributed to the interpretation of the results. The inelastic neutron scattering results as well as the static magnetic susceptibility data were obtained by Ekaterina S. Klyushina and Bella Lake. Ekaterina S. Klyushina also did the main part of the fitting analyses. She was supported by Bella Lake. The diagrammatic calculations (DBA-CUT) were performed by Benedikt Fauseweh under the supervision of Götz S. Uhrig.

\section{\begin{tabular}{l|l} 
Introduction & 7.1
\end{tabular}}

Many magnetic materials with strong electronic correlations host unconventional states of matter at low temperatures. Upon raising the temperature, there develops an interesting interplay of quantum and thermal fluctuations in which the latter will ultimately suppress the quantum mechanical coherence of such states. For potential applications in quantum devices the study of this crossover from the quantum regime at low temperatures to the classical high-temperature regime is of practical importance. Previous experimental and theoretical progress reviewed in the following paragraphs reported that strong correlations between thermally-induced excitations may lead to quantum coherence persisting even at relatively high temperatures in quantum magnets.

Such coherent behavior at elevated temperature is not expected by the conventional description of temperature effects in quantum magnets [ML71] which can be summarized as follows. In the limits of zero temperature and long wavelength, elementary excitations in quantum magnets do not interact and have an infinite lifetime. At finite temperature, the interactions between the excitations are assumed to be weak in the conventional picture. The excitations are oftentimes described as spin waves [HKHH71, TH90, Kop90]. The scattering between spin waves can be calculated in perturbation theory [TH90]. The states occupied as a consequence of the scattering processes represent an extensive portion of phase space as the excitations only interact weakly. With rising temperature the number of thermally excited spin waves grows and thus their scattering rate increases. This limits the lifetime of the excitations which is decreasing with increasing temperature. The result is a loss of coherence. The corresponding damping of spin waves results in a symmetric Lorentzian broadening of the lineshapes. The spin-wave description works best at low temperature for systems with long-range order and gapless excitations, e.g., in 
the presence of dominant nearest-neighbor antiferromagnetic (AFM) Heisenberg interactions in $\mathrm{SU}(2)$ invariant systems. Two experimentally investigated examples are $\mathrm{Rb}_{2} \mathrm{MnF}_{4}$ $\left[\mathrm{HTC}^{+} 08, \mathrm{BTL}^{+} 13\right]$ which is a two-dimensional Heisenberg antiferromagnet and the threedimensional $\mathrm{MnF}_{2}\left[\mathrm{BKHK} 6, \mathrm{BTL}^{+} 13\right]$ which has dominating $\mathrm{AFM}$ interactions. In both compounds the magnetic $\mathrm{Mn}^{2+}$ ions possess a spin of $S=5 / 2$. The thermal lineshape broadening in these materials exhibits a symmetric Lorentzian profile and is therefore in agreement with the predictions from spin-wave theory [HKHH71, TH90, Kop90]. Moreover, there is also a 2D Heisenberg antiferromagnet with spin-1/2 degrees of freedom for which such behavior has been reported $\left[\mathrm{RMC}^{+} 01\right]$.

The conventional picture of thermal decoherence described above is not valid for quantum magnets in general. There exist magnets with strong correlations between the thermally activated excitations. Thus, the available states are constrained in phase space. Gapped antiferromagnets and systems in which the excitations can be described by hard-core bosons constitute possible candidates. Among them are for instance Haldane chains [Aff89] and dimerized systems [TBR $\left.{ }^{+} 03\right]$. The present chapter is focused on 1D dimer magnets. Assuming only an AFM intradimer coupling $J_{\text {intra }}$, the system consists of isolated dimers. Each of them has a spin-singlet ground state with degenerate triplet excitations above an energy gap. Such excitations are henceforth referred to as triplons [SU03]. Upon introducing an interdimer coupling $J_{\text {intra }}$, triplons become mobile and dispersive. Imposing the condition $J_{\text {intra }} \ll J_{\text {inter }}$ guarantees a large gap compared to the bandwidth of the single-triplon dispersion. The triplons are spin $S=1$ quasiparticles. Moreover, there is a hard-core constraint for triplons. This means that only one excitation is allowed per dimer. With rising temperature the number of thermally activated triplon excitations increases $\sim \exp (-\beta \Delta)$. Due to the hard-core constraint they should scatter in a strongly correlated manner. These interactions between thermal excitations restrict the available density of states, i.e., impose phase space constraints which are not accounted for in the conventional picture. Now one may wonder whether these strong correlations have an impact on the lineshape broadening with temperature. Inelastic neutron scattering (INS) experiments on copper nitrate [TLJ $\left.{ }^{+} 12\right]$, which is a $S=1 / 2$ quasi-1D dimer-chain compound with alternating AFM couplings, revealed an increasingly asymmetric profile of the observed lineshape upon raising the temperature. The asymmetry was ascribed to the correlations between the excitations in this strongly dimerized compound. This implies that quantum mechanical coherence persists at elevated temperatures. In order to support this statement, Ref. [TLJ $\left.{ }^{+} 12\right]$ also contains theoretical results based on Refs. [ML06, JEK08] which are discussed further below.

One may ask the question whether the asymmetric lineshape broadening is a peculiarity of low-dimensional systems. Due to pronounced quantum effects in one dimension and the geometrical situation favoring scattering processes among quasiparticles [Gia04], it might be tempting to think so. However, the answer is that the occurrence of asymmetric lineshapes is not restricted to low dimensions since it has also been observed in the gapped $3 \mathrm{D}$ spin- $1 / 2$ dimer compound $\mathrm{Sr}_{3} \mathrm{Cr}_{2} \mathrm{O}_{8}$ [QCLI $\left.{ }^{+} 12, \mathrm{JQCI}^{+} 14\right]$. This suggests that it is a consequence of strong interactions between thermally activated quantum excitations.

To better understand the asymmetric temperature broadening of a coherent single-particle mode, it is useful to briefly review the main progress made in the theoretical study of finitetemperature lineshapes. The state of affairs will also motivate the DMRG calculations presented in this thesis. Previous approaches based on semiclassical approximations are only valid at temperatures $T \ll \Delta$, which are significantly lower than the spin excitation 
gap $\Delta$. They predict a symmetric Lorentzian lineshape at energies $\omega \sim \Delta$ close to the gap [SY97, DS98, DS05, RZ06]. Dynamical response functions can also be calculated using low-temperature expansions in integrable quantum field theories [EK08, EK09]. The method predicts an asymmetric lineshape broadening of a single-particle excitation for such integrable field theories. This approach may also be applied to 1D lattice models. However, dimerized spin-1/2 Heisenberg chains, as studied in this chapter, are not exactly solvable. Thus, the required matrix elements cannot be calculated from integrability constraints any more and need to be approximated perturbatively [JEK08]. Apart from the AFM-AFM dimerized chain [JEK08], the approach also predicted asymmetric lineshape broadening in a dimerized spin ladder [GKE10]. The asymmetry is found to increase with temperature in both cases. However, these results are only expected to be valid for temperatures lower than the gap $\Delta$. A full diagonalization study [ML06, Luc08] for the AFM-AFM dimerized chain also reported an increasingly asymmetric profile of the lineshape with rising temperature. Nevertheless, the accessible system sizes with this approach have been rather limited $(L=16)$. For these reasons, it would be desirable to compute the finite-temperature spectral functions of such 1D dimer systems in a controlled way also allowing for the treatment of larger systems.

Such calculations with quantitative predictive power are the main goal of this chapter and are intended to put the occurrence of an asymmetric lineshape on more firm grounds. To this end, the finite-temperature DMRG method working directly in the frequency domain and developed in this thesis is employed, see Sec. 3.6. The material investigated in this chapter is the quasi-1D dimer compound $\mathrm{BaCu}_{2} \mathrm{~V}_{2} \mathrm{O}_{8}$ [VMB90]. The DMRG calculations are compared to high-resolution inelastic neutron scattering (INS) measurements for $\mathrm{BaCu}_{2} \mathrm{~V}_{2} \mathrm{O}_{8}$ and theoretical results obtained by the Brückner approach on top of continuous unitary transitions (DBA-CUT) [FSU14, FU15]. By this comparison between experimental and theoretical results, the presence of strongly correlated behavior at elevated temperatures is studied. A second main goal of this chapter is to identify the Hamiltonian of $\mathrm{BaCu}_{2} \mathrm{~V}_{2} \mathrm{O}_{8}$. So far there have been no measurements of the spin excitation spectra in $\mathrm{BaCu}_{2} \mathrm{~V}_{2} \mathrm{O}_{8}$. Therefore, mainly static susceptibility measurements [SYK02, HKI04, HTI06, GPB ${ }^{+} 05$, SMD08] and band structure calculations [KW06, SMD08] have obtained conflicting results about the weaker interdimer interaction. Its strength was predicted to lie in the range between $0 \%$ and $20 \%$ of the intradimer coupling. However, a comparison of INS data and DMRG calculations for $\mathrm{BaCu}_{2} \mathrm{~V}_{2} \mathrm{O}_{8}$ will reveal a weak ferromagnetic (FM) interdimer coupling. The material thus represents a complementary system to the purely AFM dimer-chain compound copper nitrate. Therefore, it is also investigated whether the correlated behavior in gapped dimer systems depends on the sign of the interdimer coupling. Another subject studied by finite-temperature DMRG calculations is the finite low-frequency intensity arising from intraband transitions which has been observed for copper nitrate [TLJ $\left.{ }^{+} 12\right]$.

This chapter is structured as follows: In Sec. 7.2, the state of affairs concerning the compound $\mathrm{BaCu}_{2} \mathrm{~V}_{2} \mathrm{O}_{8}$ is presented. The Hamiltonian of $\mathrm{BaCu}_{2} \mathrm{~V}_{2} \mathrm{O}_{8}$ is determined in Sec. 7.3. Section 7.4 presents the main results verifying the asymmetric lineshape in $\mathrm{BaCu}_{2} \mathrm{~V}_{2} \mathrm{O}_{8}$ at finite-temperatures by a comparison of experimental and theoretical results. It also contains DMRG calculations for the thermally activated excitations at low frequency which are predicted to occur in $\mathrm{BaCu}_{2} \mathrm{~V}_{2} \mathrm{O}_{8}$. A conclusion of the present chapter as well as perspectives for future research are given in Sec. 7.5. 


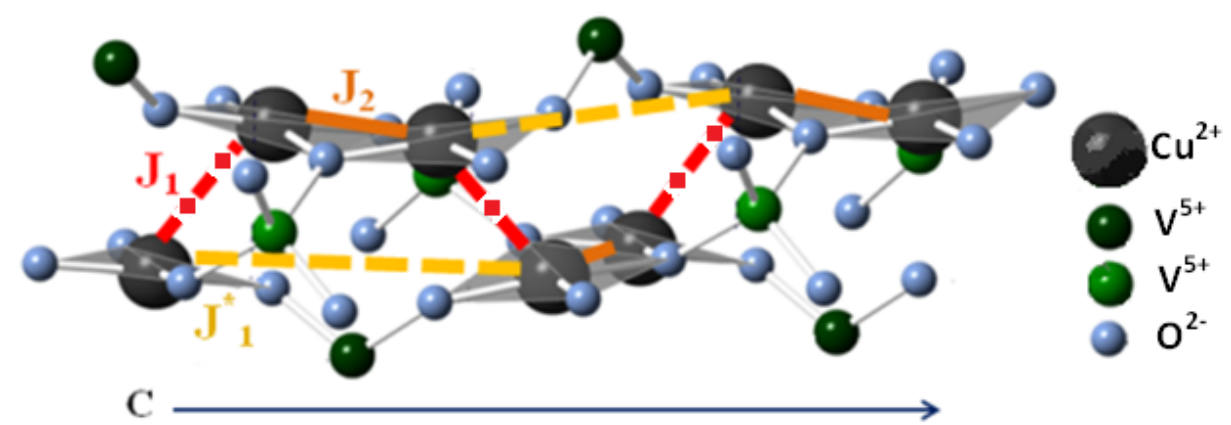

Figure 7.1: (Taken from Ref. $\left[\mathrm{KTF}^{+} 16\right]$.) Key structural element of $\mathrm{BaCu}_{2} \mathrm{~V}_{2} \mathrm{O}_{8}$ showing the two proposed models of the dimerized chain along the $c$ axis: first model with exchange paths $J_{1}^{*}$ (dashed line) and $J_{2}$ (solid line) resulting in two independent non-interacting dimerized linear chains; second model with exchange paths $J_{1}$ (dash-dotted line) and $J_{2}$ (solid line) leading to a single dimerized screw chain. The $\mathrm{Ba}^{2+}$ ions are omitted.

\section{\begin{tabular}{l|l} 
The compound $\mathrm{BaCu}_{2} \mathrm{~V}_{2} \mathrm{O}_{8}$ & 7.2
\end{tabular}}

The investigated compound $\mathrm{BaCu}_{2} \mathrm{~V}_{2} \mathrm{O}_{8}$ [VMB90] has a tetragonal crystal structure of space group $\mathrm{I} \overline{4} 2 \mathrm{~d}$. The lattice parameters are $a=b=12.744 \AA$ and $c=8.148 \AA$. Figure 7.1 shows the key structural element of $\mathrm{BaCu}_{2} \mathrm{~V}_{2} \mathrm{O}_{8}$. The material essentially consists of alternating chains of magnetic $\mathrm{Cu}^{2+}$ ions with spin $S=1 / 2$ that are coordinated by $\mathrm{O}^{2-}$ ions in square-planar geometry. These $\mathrm{CuO}_{4}$ plaquettes form edge-sharing pairs which rotate about the $c$ axis and are oriented with the $c$ axis lying within the plaquettes as shown in Fig. 7.1.

Previous static magnetic susceptibility [SYK02, HKI04, HTI06, GPB ${ }^{+}$05, SMD08], specific heat [HKI04] and ${ }^{51} \mathrm{~V}$ nuclear magnetic resonance $\left[\mathrm{GPB}^{+} 05\right.$, LX05] measurements revealed a non-magnetic ground state. The magnetic ground-state excitations were found above a gap of $\Delta \approx 31.0-40.5 \mathrm{meV}$. This implies that the $\mathrm{Cu}^{2+}$ ions are coupled into dimers by a dominant AFM intradimer interaction. The dimerization leads to a spinsinglet ground state and gapped triplon excitations.

However, there are conflicting results about a possible weaker interdimer interaction. Based on static susceptibility measurements on powder samples and supporting electronic structure calculations, Ref. [SMD08] concludes in favor of an isolated dimer model. This model is also in agreement with previous NMR results [LX05]. In contradiction, other experimental works predict a weak AFM interdimer coupling of up to $20 \%$ of the intradimer coupling [HKI04, HTI06, GPB ${ }^{+} 05$, LX05].

The exchange paths, modeled as the intradimer $\left(J_{\text {intra }}\right)$ and interdimer $\left(J_{\text {inter }}\right)$ couplings in the Hamiltonian (4.18), are strongly debated in the literature [HTI06, KW06, SMD08]. These paths are sketched in Fig. 7.1. The magnetic interaction $J_{2}$ between the two $\mathrm{Cu}^{2+}$ ions within the edge-sharing plaquettes is mediated via the superexchange path $\mathrm{Cu}-\mathrm{O}-$ $\mathrm{Cu}$ [KW06], which is marked by $J_{2}$ and associated with the exchange coupling $J_{\text {inter }}$ in the Hamiltonian (4.18). Moreover, there are also proposed super-superexchange paths $(\mathrm{Cu}-\mathrm{O}-\mathrm{V}-\mathrm{O}-\mathrm{Cu})$ denoted by $J_{1}$ and $J_{1}^{*}$ in Fig. 7.1 [KW06]. These enter the model in Eq. (4.18) as $J_{\text {intra }}$. Two models for $\mathrm{BaCu}_{2} \mathrm{~V}_{2} \mathrm{O}_{8}$ have been suggested. The first, assuming the paths $J_{2}$ and $J_{1}^{*}$, gives rise to almost straight alternating double chains parallel to the $c$ axis [HKI04]. The second, in which $J_{1}$ and $J_{2}$ are the exchange paths, couples the $\mathrm{Cu}^{2+}$ ions into alternating screw chains [KW06]. The second model is favored by 
two electronic structure investigations which predict that $J_{1}$ and $J_{2}$ are both AFM with ratio $J_{2} / J_{1}$ of 0.16 [KW06] or $0.05\left[\mathrm{GPB}^{+} 05\right]$, while $J_{1}^{*}$ is much weaker. However, the approach [WKD03] used for the spin dimer analyses in Ref. [KW06] neglects a possible FM intradimer coupling as an assumption.

In summary, the above findings of previous works leave open questions concerning the microscopic model of $\mathrm{BaCu}_{2} \mathrm{~V}_{2} \mathrm{O}_{8}$. The first one is whether the compound is described by isolated dimers or rather a strongly alternating chain. The second issue would be to reliably determine the sign (AFM versus FM) and magnitude of a possible interdimer interaction. The goal of the following Sec. 7.3 is to shed new light on these issues and to identify the Hamiltonian.

\section{Identification of the Hamiltonian $\mid 7.3$}

\section{Static magnetic susceptibility $\mid 7.3 .1$}

So far most measurements of the static magnetic susceptibility have only been obtained for temperatures up to 300-400 K [SYK02, GPB ${ }^{+}$05, HTI06, SMD08]. As noted by Ref. [SMD08], there is a problem concerning the data analysis in Ref. [HKI04] which has been the only work providing high-temperature data up to $900 \mathrm{~K}$ for the static magnetic susceptibility. However, in order to distinguish an AFM interdimer coupling from a FM one, the analysis of the high-temperature region is crucial since the different behavior is most pronounced there [Bü03]. Therefore, the static magnetic susceptibility $\chi$ is measured over the temperature range 2-900 $\mathrm{K}$ for a magnetic field parallel $H \| c$ and perpendicular $H \perp c$ to the $c$ axis. Both experimental curves are of very similar shape, although their amplitude for $H \perp c$ is about $15 \%$ larger than for the other direction. This implies an anisotropic $g$ factor in $\mathrm{BaCu}_{2} \mathrm{~V}_{2} \mathrm{O}_{8}$. Moreover, Figure 7.2 shows fits to the experimental susceptibility data. The fitting function is of the form

$$
\begin{aligned}
\chi_{\text {observed }}(T) & =\chi_{0}+\chi_{\text {imp }}(T)+\chi_{\text {int. dimer }}(T) \\
& =\chi_{0}+\frac{C_{\text {imp }}}{T-\theta}+\frac{3 C / T}{3+\mathrm{e}^{J_{\text {intra }} /\left(k_{\mathrm{B}} T\right)}+J_{\text {inter }} / T}
\end{aligned}
$$

which contains the susceptibility $\chi_{\text {int.dimer }}(T)$ for a model of coupled dimers treated in a molecular field approximation [Joh97, SJ07]. See Appendix B.1 for the derivation. Equation (7.2) also includes a contribution $\chi_{\mathrm{imp}}$ from paramagnetic impurities. The Weiss temperature of which is denoted by $\theta$ and $C_{\mathrm{imp}}$ is the Curie constant of the impurities. $\chi_{0}$ is a temperature independent offset. The best fit to the experimental data yields the fit results $J_{\text {intra }}=39.8 \pm 0.13 \mathrm{meV}, J_{\text {inter }}=-9.87 \pm 2.64 \mathrm{meV}, g_{\| c}=2.09$, and $g_{\perp c}=2.27$, which suggest a FM interdimer coupling. The $g$ factors obtained from the experimental data are calculated from the fitting result for the Curie constant $C$ of the magnetic atoms via the relation [Joh97]

$$
C=\frac{N_{\text {atoms }} g^{2} \mu_{\mathrm{B}}^{2} S(S+1)}{3 k_{\mathrm{B}}}
$$

To further investigate this issue, Figure 7.2 also includes susceptibility data for the Hamiltonian (4.18) obtained via an imaginary-time evolution using DMRG and the purification 

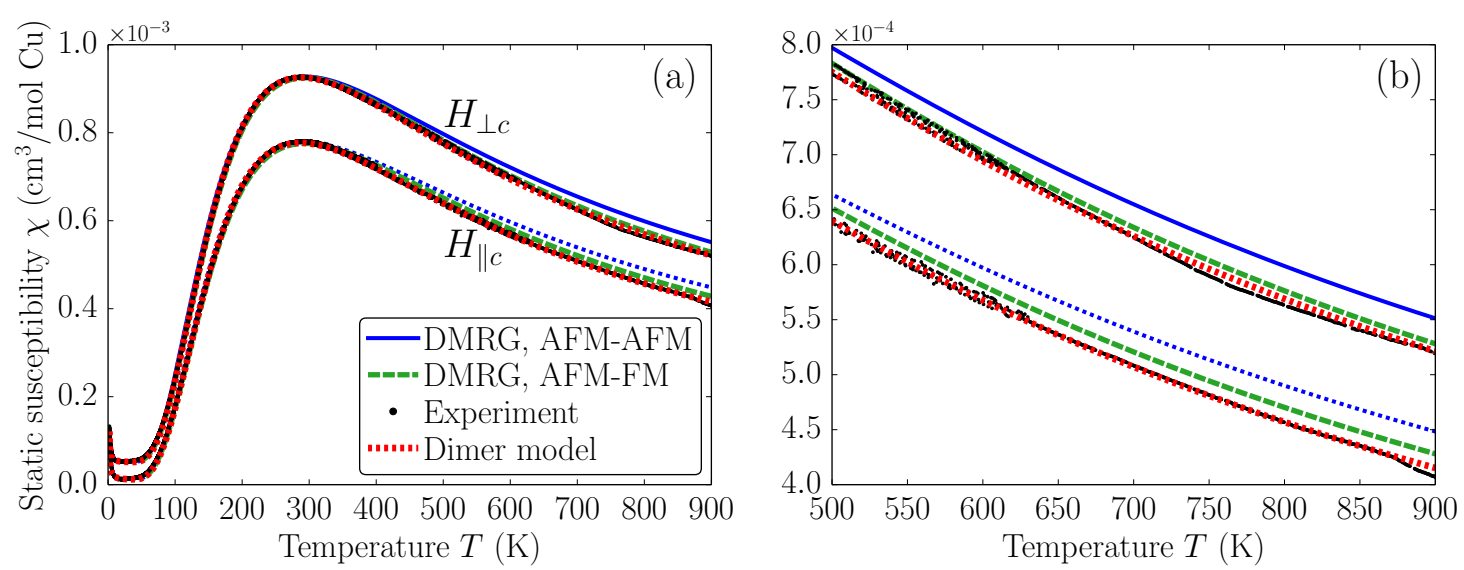

Figure 7.2: (Adapted from Ref. $\left[\mathrm{KTF}^{+} 16\right]$.) (a) Static magnetic susceptibility $\chi$ as a function of the temperature $T$ for a magnetic field of magnitude $1 \mathrm{~T}$ applied parallel $\left(H_{\| c}\right)$ and perpendicular $\left(H_{\perp c}\right)$ to the $c$ axis. The dotted red line represents the susceptibility in Eq. (7.2) calculated for a model of weakly coupled dimers. Here the $g$ factors and exchange constants were fitted $\left(J_{\text {intra }}=39.8 \pm 0.13 \mathrm{meV}, J_{\text {inter }}=-9.87 \pm 2.64 \mathrm{meV}, g_{\| c}=2.09, g_{\perp c}=2.27\right)$. The DMRG results are the solid lines (AFM-AFM model) and the dashed (AFM-FM model) lines. Here the exchange couplings are not fitted but rather adopted from Tab. 7.1 below. Scaling the DMRG data on top of the experimental results yields anisotropic $g$ factors of $g_{\| c}=2.14 \pm 0.015$ and $g_{\perp c}=2.29 \pm 0.015$ for the AFM-FM model. Panel (b) focuses on the high-temperature behavior where the differences between the AFM-AFM and the AFM-FM case are most pronounced, which can be seen from the DMRG data. The experimental measurements and the fit analysis were performed by Ekaterina S. Klyushina. The DMRG data were obtained in this thesis.

trick (see Sec. 3.6.2). ${ }^{1}$ These calculations are performed for both the AFM-AFM and AFM-FM model using the corresponding exchange couplings taken from Tab. 7.1 below. Here only the $g$ factors were determined by scaling the AFM-FM DMRG results to the experimental data yielding $g_{\| c}=2.14 \pm 0.015$ and $g_{\perp c}=2.29 \pm 0.015$. Thus, there is good agreement with the $g$ factors obtained for the coupled-dimer model above. Moreover, in $\mathrm{BaCu}_{2} \mathrm{~V}_{2} \mathrm{O}_{8}$ the plaquettes contain the $c$ axis and rotate about it. Therefore, one considers the $g$ factors parallel $g_{\| \text {plaq }}=g_{\| c}=2.12 \pm 0.03$ and perpendicular $g_{\perp \text { plaq }}=2 g_{\perp c}-g_{\| c}=2.44 \pm 0.03$ to the plaquettes. For these values the $g$ factors obtained from the experimental and DMRG data are averaged. The results are for instance in agreement with the related material $\mathrm{BaCuSi}_{2} \mathrm{O}_{6}$ where $g_{\| \text {plaq }} \approx 2.05$ and $g_{\perp \text { plaq }} \approx 2.31$ $\left[\mathrm{ZWK}^{+} 06\right]$.

To answer the question which model, AFM-AFM or AFM-FM, gives a better description, the focus is on the high-temperature data in the right panel of Fig. 7.2. Here the AFMFM DMRG results are considerably closer to the experimental data giving another hint that the interdimer coupling could indeed be FM. As also concluded previously [HTI06], it is difficult to determine the Hamiltonian for $\mathrm{BaCu}_{2} \mathrm{~V}_{2} \mathrm{O}_{8}$ solely based on thermodynamic measurements. To this end, inelastic neutron scattering experiments probing the dynamical spin structure factor of sufficiently large single crystals are presented in Sec. 7.3.2 and compared to DMRG calculations for both models.

\footnotetext{
${ }^{1}$ In contrast to the measurement which is conducted in the presence of a magnetic field of $1 \mathrm{~T}$, there is no magnetic field included in the DMRG calculations. This is justified since the applied field is negligible compared to the energy scale of the dominant exchange coupling.
} 


\section{Magnetic excitation spectra at $T=0 \quad 7.3 .2$}

In the present section the Hamiltonian of $\mathrm{BaCu}_{2} \mathrm{~V}_{2} \mathrm{O}_{8}$ shall be finally deduced. It thus aims at a comparison between the magnetic excitation spectra measured by INS and DMRG results for the dynamical spin structure factor for both the AFM-AFM and the AFM-FM model. The experiments are conducted at a temperature of $T=5 \mathrm{~K}$ which is sufficiently low in order to compare the magnetic excitation spectra to zero-temperature DMRG calculations. Before the results are presented, the technical details of these DMRG calculations are briefly discussed.

\section{Details of the DMRG calculations}

The DMRG computations are performed directly in the frequency domain for 1D systems of finite size and open boundary conditions (OBCs). MPS-based Chebyshev expansions at zero temperature are used, see Sec. 3.5.4. It is important to take account of the crystal structure of $\mathrm{BaCu}_{2} \mathrm{~V}_{2} \mathrm{O}_{8}$ since there is an experimental hint that the weaker dispersion in Fig. 7.3(a) below might be engendered by the screw-chain geometry of the compound. In order to include the effect of the crystal structure in the longitudinal dynamical spin structure factor, the real positions $R_{j}$ of $N_{\text {atom }}=80$ copper atoms along one screw chain are used in the Fourier transform of the spin operator

$$
S_{Q}^{z}=\frac{1}{\sqrt{N_{\text {atom }}}} \sum_{j} \mathrm{e}^{-2 \pi i Q \cdot R_{j}} S_{j}^{z} .
$$

The momentum $Q$ is specified by Miller indices $Q=(\mathrm{H}, \mathrm{K}, \mathrm{L})$ and $S_{j}^{z}$ is the $z$ component of the local spin operator acting at site $j$. With this, the zero-temperature longitudinal dynamical structure factor is given by

$$
\begin{aligned}
& S_{z z}^{T=0}(\omega, Q)=\sum_{n}\left|\left\langle E_{n}\left|S_{Q}^{z}\right| E_{0}\right\rangle\right|^{2} \delta\left(\omega-\left(E_{n}-E_{0}\right)\right) \\
& =\left\langle E_{0}\left|S_{-Q}^{z} \delta\left(\omega-\left(H-E_{0}\right)\right) S_{Q}^{z}\right| E_{0}\right\rangle \\
& =\sum_{j, l} \mathrm{e}^{2 \pi i Q \cdot\left(R_{l}-R_{j}\right)} \underbrace{\left\langle E_{0}\left|S_{l}^{z} \delta\left(\omega-\left(H-E_{0}\right)\right) S_{j}^{z}\right| E_{0}\right\rangle}_{=: G_{l, j}(\omega)} .
\end{aligned}
$$

Here $\left|E_{n}\right\rangle$ and $E_{n}$ denote the eigenstates and eigenvalues of the Hamiltonian $H$. Equations (7.5b) and (7.5c) now offer two different schemes for the computation of spectral functions via MPS-based Chebyshev expansions. In the first case of Eq. (7.5b), $Q$ is specified prior to one single calculation in momentum space. More flexibility is provided by the scheme in Eq. (7.5c). Here the dynamical correlation functions $G_{l, j}(\omega)$ are computed individually in real space giving access to arbitrary momenta in the postprocessing stage, at the expense of an increasing computational effort by a factor $\sim N_{\text {atom. }}$. Moreover, the reflection symmetry of the system is also exploited for $G_{l, j}(\omega)$. The latter computation scheme is therefore advantageous in order to obtain results along various directions in $Q$ space as shown in Fig. 7.3 below. For these zero-temperature results a maximal internal MPS bond dimension of $m=150$ is retained. Note that the nearly Gaussian broadening introduced in the Chebyshev expansions is chosen equal to the experimental resolution. As the broadening depends inversely on the number of Chebyshev iterations, see Eq. (3.95), this also gives a stopping criterion for the calculations. In each Chebyshev iteration the 
error resulting from the variational compression is $\epsilon_{\text {compr }}<10^{-9}$. Since there are two screw chains with different winding orientation in each $\mathrm{BaCu}_{2} \mathrm{~V}_{2} \mathrm{O}_{8}$ unit cell, the results are obtained as a superposition of both screw chains.

\section{Comparison between DMRG and INS experiments}

Figures 7.3(a)-(b) present INS data measured along two directions in the (H, 0, L) plane at $T=5 \mathrm{~K}$. Along $(6,0, \mathrm{~L})$ the magnetic excitation spectrum consists of two gapped branches dispersing over the energy range $35.37 \pm 0.05 \mathrm{meV}$ to $45.56 \pm 0.05 \mathrm{meV}$, as shown in Fig. 7.3(a). The modes have the same periodicity and bandwidth, but are shifted with respect to each other by half a period and alternate in intensity. The two branches are dispersionless along the $\mathrm{H}$ direction, which can be inferred from the INS data in Fig. 7.3(b). The same is also true for the $\mathrm{K}$ direction. Both modes in Fig. 7.3(a), the strong and the weak one, are in very good agreement with the fifth-order expansion of the one-triplon dispersion in Eq. (4.24) obtained for the alternating Heisenberg chain. Assuming either an AFM-AFM (dashed line) or an AFM-FM alternating chain (solid line), the fifth-order expression for the dispersion is plotted in Fig. 7.3(a).

These results show that $\mathrm{BaCu}_{2} \mathrm{~V}_{2} \mathrm{O}_{8}$ is a highly dimerized 1D quantum magnet in which the dimers are coupled along the $c$ axis whereas the coupling in the $a b$ plane is absent or negligibly small. Moreover the presence of a structure factor with two modes implies that these chains are not straight. In addition to the following arguments, this can also be concluded from the DMRG results discussed further below. The extracted value of the alternating chain periodicity $(d=4.04 \pm 0.04 \AA)$ is the same for both modes and is half the lattice parameter $c$. This periodicity corresponds to the alternating screw chain model assuming the exchange paths denoted by $J_{1}$ and $J_{2}$, whereas the linear chain model $\left(J_{1}^{*}\right.$ and $J_{2}$ ) can be excluded because it would have a periodicity of $d=c=8.124 \AA$. Thus, the INS results confirm the exchange path predicted by electronic structure calculations [KW06]. However, from this one cannot determine which of the two exchange paths is $\mathrm{FM},{ }^{2}$ it is most likely that $J_{1}=J_{\text {intra }}$ is AFM, while $J_{2}=J_{\text {inter }}$ is FM. This assumption is based on band structure calculations predicting that the super-superexchange path $J_{1}(\mathrm{Cu}-\mathrm{O}-\mathrm{V}-\mathrm{O}-\mathrm{Cu})$ provides the strongest AFM interaction [KW06, SMD08] and that the bridge angle of the $J_{2}$ superexchange path $(\mathrm{Cu}-\mathrm{O}-\mathrm{Cu})$ is $94^{\circ}$ [KW06]. This further supports the current finding as the predicted angle is very close to the crossover from AFM to FM according to the Goodenough-Kanamori-Anderson rules [And50, Kan59, Goo60].

As a next step, the values of $J_{\text {inter }}$ and $J_{\text {intra }}$ are determined via a fit of the fifth-order expansion of the one-triplon dispersion in Eq. (4.24) to high-resolution energy scans at the dispersion minima and maxima. In this fitting analysis both the AFM-AFM and AFMFM models were considered leading to the fit results summarized in Tab. 7.1. Using these values for the couplings as an input $\left(J_{\text {intra }}=1, J_{\text {inter }}=J_{2} / J_{1}\right)$, DMRG computations for the dynamical spin structure factor at $T=0$ were performed for both models.

To distinguish between the AFM-AFM and AFM-FM alternating screw-chain models, these zero-temperature DMRG calculations provide results along the $(6,0, \mathrm{~L})$ and $(\mathrm{H}, 0,5)$ directions for the AFM-AFM (Figs. 7.3(c)-(d)) and the AFM-FM (Figs. 7.3(e)-(f)) models. In both cases gapped modes are found, matching the experimental data in terms of energy and periodicity. However, only the AFM-FM model agrees with the observed intensity while the AFM-AFM chain is clearly wrong since the strong and the weak modes

\footnotetext{
${ }^{2}$ It is also not possible to conclude this from the DMRG calculations below.
} 

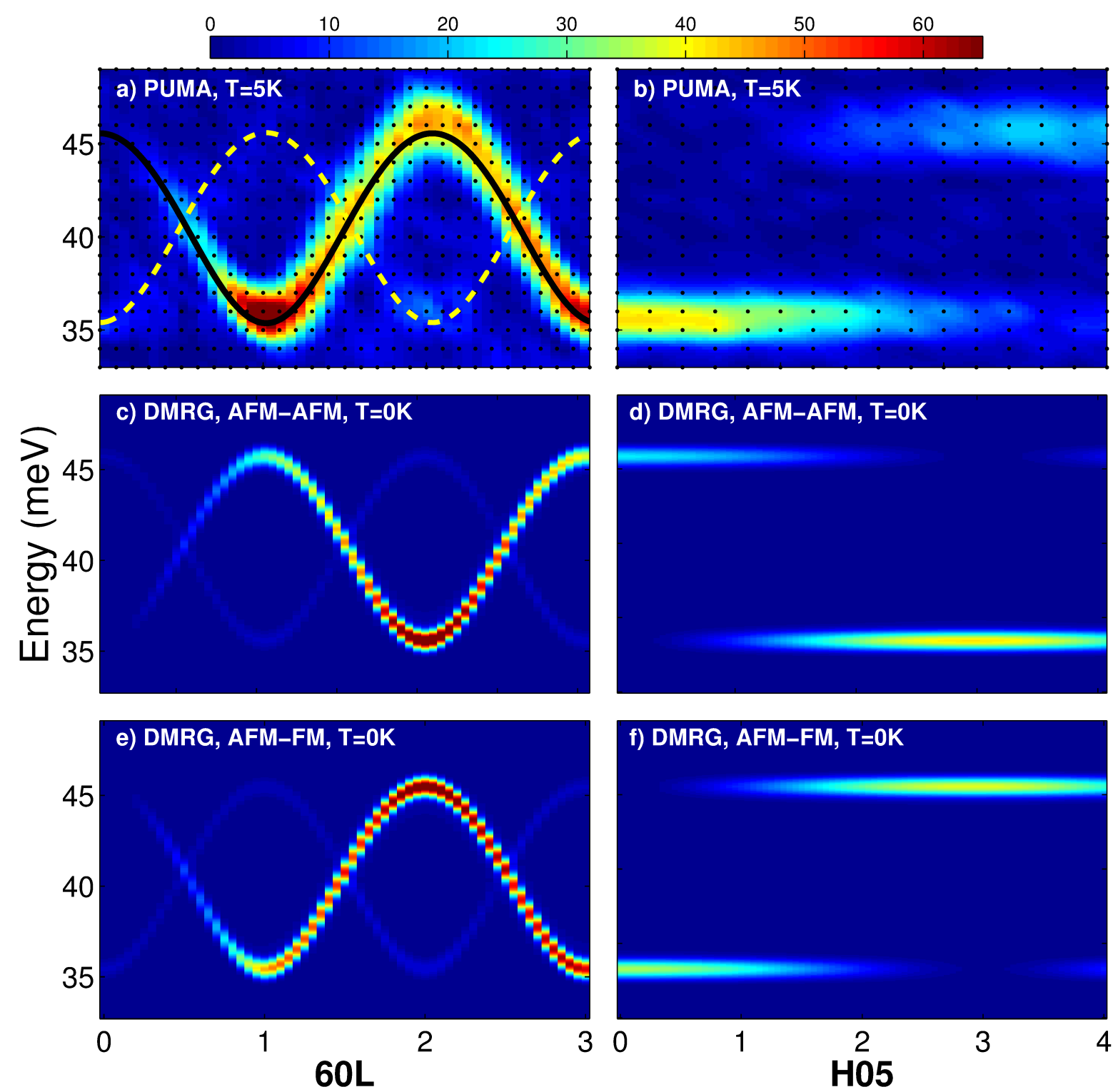

Figure 7.3: (Adapted from Ref. $\left[\mathrm{KTF}^{+} 16\right]$.) Panels (a) and (b) show INS results contributed by the experimental collaborators. Panels (c)-(f) contain DMRG data obtained in this thesis. Background-subtracted INS data along (a) $(6,0, \mathrm{~L})$ and $(\mathrm{b})(\mathrm{H}, 0,5)$ at $T=5 \mathrm{~K}$. The dashed and solid lines show the one-triplon dispersion to fifth order in $J_{2} / J_{1}$ for the AFM-AFM model $\left(J_{1}=40.75 \pm 0.02 \mathrm{meV}, J_{2}=9.16 \pm 0.10 \mathrm{meV}\right)$ and AFM-FM $\left(J_{1}=40.92 \pm 0.01 \mathrm{meV}\right.$, $J_{2}=-11.97 \pm 0.10 \mathrm{meV}$ ) model, respectively. The zero-temperature dynamical structure factor is calculated by DMRG for the AFM-AFM screw-chain model along (c) $(6,0, \mathrm{~L})$ and (d) $(\mathrm{H}, 0,5)$. Furthermore, this quantity is also computed for the AFM-FM screw-chain model along (e) $(6,0, \mathrm{~L})$ and $(\mathrm{f})(\mathrm{H}, 0,5)$. In both cases, the calculations adopt the values for the exchange couplings specified above as well as in Tab. 7.1. The DMRG results take into account the positions of the $\mathrm{Cu}^{2+}$ ions, see Eq. (7.4). 


\begin{tabular}{c|c|c|c}
\hline \hline model & $J_{1}(\mathrm{meV})$ & $J_{2}(\mathrm{meV})$ & $J_{2} / J_{1}$ \\
\hline AFM-AFM & $40.75 \pm 0.02$ & $9.16 \pm 0.10$ & 0.225 \\
AFM-FM & $40.92 \pm 0.01$ & $-11.97 \pm 0.10$ & -0.29 \\
\hline \hline
\end{tabular}

Table 7.1: Fit results for the intradimer and the weaker interdimer exchange couplings obtained by a fit of the fifth-order expansion of the one-triplon dispersion (see Eq. (4.24)) to high-resolution energy scans at the dispersion minima and maxima.

are interchanged with respect to the experiment. The DMRG results thus confirm the presence of an FM interdimer coupling, which represents one of the main results. This is in agreement with the static magnetic susceptibility data which has been analyzed in Sec. 7.3.1.

Moreover, another important result is that the DMRG calculations help to undoubtedly relate the origin of the low-intensity dispersion branches to the screw-chain geometry. These weak features only appear if one takes into account the corresponding crystal structure in the Fourier transform (7.4). If the Fourier transform for a linear chain is used instead, the low-intensity dispersions are not visible. Consequently, they are not engendered by interchain coupling.

\section{\begin{tabular}{l|l} 
Finite-temperature results & 7.4
\end{tabular}}

Having determined the Hamiltonian of $\mathrm{BaCu}_{2} \mathrm{~V}_{2} \mathrm{O}_{8}$ in the previous sections, this knowledge is used to investigate the persistence of strongly correlated behavior at elevated temperatures. Since materials described by an alternating AFM-FM chain are scarce, $\mathrm{BaCu}_{2} \mathrm{~V}_{2} \mathrm{O}_{8}$ grants the opportunity to study the effect of temperature on a new dimer system with weak FM interdimer coupling.

\section{Correlation length $\mid 7.4 .1$}

In Fig. 7.4(a) both the temperature and distance dependence of the correlation functions $\left|\left\langle S_{\frac{L}{2}}^{z} S_{\frac{L}{2}+j}^{z}\right\rangle\right|$ for the AFM-FM dimer model with $J_{\text {inter }} / J_{\text {intra }}=-0.29$ are studied. Note that these data are shown in dependence of the inverse temperature. The correlations are evaluated in the thermal states obtained by TDVP imaginary-time evolution with an internal MPS bond dimension of $m=150$. As expected for a system of weakly coupled dimers, the correlations decrease rapidly with increasing distance. In particular, at inverse temperatures $\beta<3$ (i.e. $T / J_{\text {intra }}>1 / 3$ ) the decay of the correlations becomes even faster with increasing temperature. This implies a shorter correlation length towards higher temperatures which is expected due to increasing thermal fluctuations. At first sight, for $\beta>3$ the decay of the correlations appears to be almost identical over a wide range of temperatures in Fig. 7.4(a). However, this has to be analyzed in more detail.

To this end, the correlation length $\xi$ is determined by fits of the form $\sim \exp (-j / \xi)$ to the data in Fig. 7.4(a). Each fit is obtained at a fixed temperature. The results for $\xi$ are displayed in Fig. 7.4(b) and allow to assess the extent of the low temperature region even better. At low temperatures, $\xi$ is found very close to the correlation length calculated in 
the ground state of a 50-site chain. There appears to be a very weak maximum. However, its presence is not guaranteed due to the fitting errors. Upon raising the temperature, the correlation length decreases. The data points suggest a monotonic decrease which is steepest around $T / J_{\text {intra }} \sim 1 / 2$.
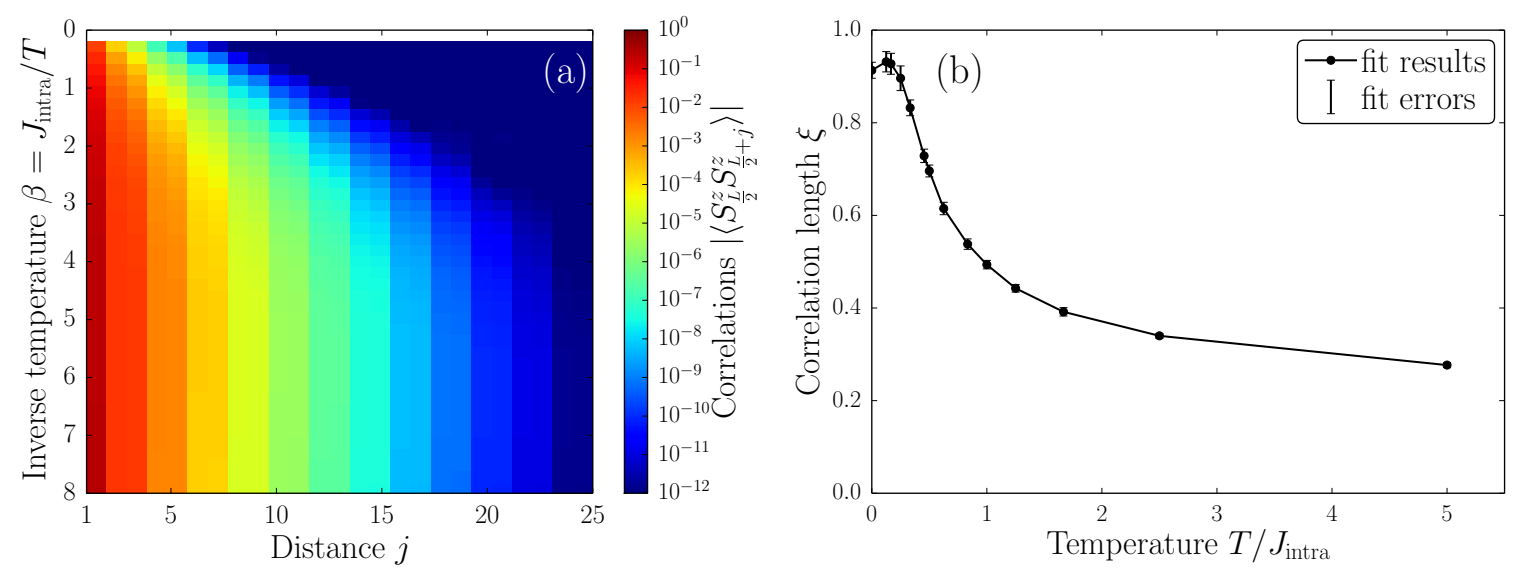

Figure 7.4: DMRG results for the AFM-FM dimer model $\left(J_{\text {inter }} / J_{\text {intra }}=-0.29\right)$ : (a) Correlations functions $\left|\left\langle S_{\frac{L}{2}}^{z} S_{\frac{L}{2}+j}^{z}\right\rangle\right|$ between the center of a chain of $L=50$ sites and spins at a distance of $j$ lattice spacings plotted in dependence of the inverse temperature $\beta=J_{\text {intra }} / T$. (b) Temperature dependence of the correlation length determined from fits of the form $\sim \exp (-j / \xi)$ to the data in panel (a). The weak maximum in $\xi(T)$ at low temperatures should not be overinterpreted since due to finite-size effects fitting errors are most pronounced in this temperature range. As expected, these errors decrease with increasing temperature.

\section{Temperature-induced asymmetric lineshape broadening}

Prior to presenting the DMRG computations for the dynamical spin structure factor at $T>0$ which represent a main result of this thesis, the INS measurements are discussed. Apart from the experimental results, one focus of this discussion is an explanation of the fitting analysis used to quantify the asymmetric lineshape broadening at elevated temperatures. This explanation is important since the same analysis is also applied to the DMRG results.

\section{Details of the DMRG calculations}

At $T>0$, the finite-temperature approach developed in this thesis is used (see Sec. 3.6). Here it is implemented as a Chebyshev expansion for the Liouville operator in an MPS framework. At finite temperature, it is computationally more expensive to compute the Chebyshev moments (see Sec. 3.6.4). Therefore, one calculates only $N=1000$ moments using a higher MPS bond dimension $(m=250)$ than at $T=0$, leading to a compression error of $\epsilon_{\text {compr }} \lesssim 10^{-4}$. Here the calculation proceeds directly in momentum space for a specific momentum $Q$, i.e., by means of the computation scheme already presented for $T=$ 0 in Eq. (7.5b). The scheme is chosen since a subsequent extrapolation of the Chebyshev moments by linear prediction is usually performed in momentum space [BSW09]. Linear prediction (see Sec. 3.5.4) is used to enhance the resolution. A more detailed presentation of the linear prediction results is given in Appendix B.4. Due to the Fourier transform in 
Eq. (7.4) complex arithmetics are used. In order to assess the quality of the extrapolation at a given temperature, the input parameters for the linear prediction are varied, e.g., the number of computed Chebyshev moments and the fitting interval. These data sets produced by linear prediction were then fitted by the function in Eq. (7.10) below. The resulting fit parameters for the Lorentzian width $W_{L}$ and asymmetry $\alpha$ displayed a slight dependence on the fit interval at a fixed temperature. The error estimates shown in Fig. 7.5(g) and 7.5(h) represent the maximal deviation found. See Appendix B.4 for the analysis of the linear prediction results. At $T>0$, only the atom positions of a single screw chain consisting of $N_{\text {atom }}=40 \mathrm{Cu}^{2+}$ ions were used for the Fourier transform in Eq. (7.4) since the effect of a different winding orientation is very small.

\section{Experimental results and fitting analysis}

In order to study the effect of temperature, INS energy scans of high resolution are obtained for a wide range of temperatures of up to $200 \mathrm{~K}$. They are performed at the dispersion minima, $(6,0,1)$ and $(8,0,0)$, where some of the most pronounced deviations from the symmetric Lorentzian lineshape are expected [JEK08, $\left.\mathrm{TLJ}^{+} 12, \mathrm{QCLI}^{+} 12, \mathrm{JQCI}^{+} 14\right]$. The background-subtracted INS data is shown in Figs. 7.5(a)-(f). First of all, it is observed that the measured lineshape broadens with increasing temperature. Note that the vertical axes in Figs. 7.5(a)-(f) have a different range in each panel. The lineshapes look more and more asymmetric as well as weighted towards the center of the band with increasing temperature.

In a first step it can be shown that the lineshape at elevated temperatures, $175 \mathrm{~K}$ and $200 \mathrm{~K}$, cannot be described by a symmetric Lorentzian profile. The idea pursued here is to fit a symmetric Lorentzian to the data. However, one also needs to take into account the resolution function $R(E)$ of the experiment. To determine $R(E)$, one assumes that at the base temperature of $3.5 \mathrm{~K}$ the observed broadening is only attributed to resolution broadening. In other words, the effect of thermal broadening is neglected. To this end, a slightly asymmetric Gaussian function $G_{\gamma}(E)$ of the form

$$
G_{\gamma}(E)=\frac{1}{\sqrt{2 \pi}\left(\frac{W_{G}}{2 \sqrt{2 \ln (2)}}+\gamma\left(E-E_{\text {peak }}\right)\right)} \exp \left(\frac{-\left(E-E_{\text {peak }}\right)^{2}}{2\left(\frac{W_{G}}{2 \sqrt{2 \ln (2)}}+\gamma\left(E-E_{\text {peak }}\right)\right)^{2}}\right)^{2}
$$

is fitted to the experimental data at base temperature in Fig. 7.5(a). Here $E$ denotes the energy variable. The Gaussian asymmetry parameter $\gamma$ introduces an asymmetric profile with respect to the peak position $E_{\text {peak }}$ of the excitation. This represents a modification to the ordinary Gaussian full width at half maximum (FWHM) $W_{G}$. From the fit, it turns out that the resolution broadening of the experiment is well described by Eq. (7.6), i.e., $R(E)=G_{\gamma}(E)$. The determined values for $W_{G}=0.89 \pm 0.03 \mathrm{meV}$ and $\gamma=0.1 \pm 0.04$ are fixed for the analysis at higher temperatures. Setting $\gamma=0$ for the fit yields a Gaussian broadening of $W_{G}=0.863 \pm 0.010 \mathrm{meV}$. Therefore, the theoretical results in Fig. 7.5, including the DMRG data, are also presented at this resolution. 

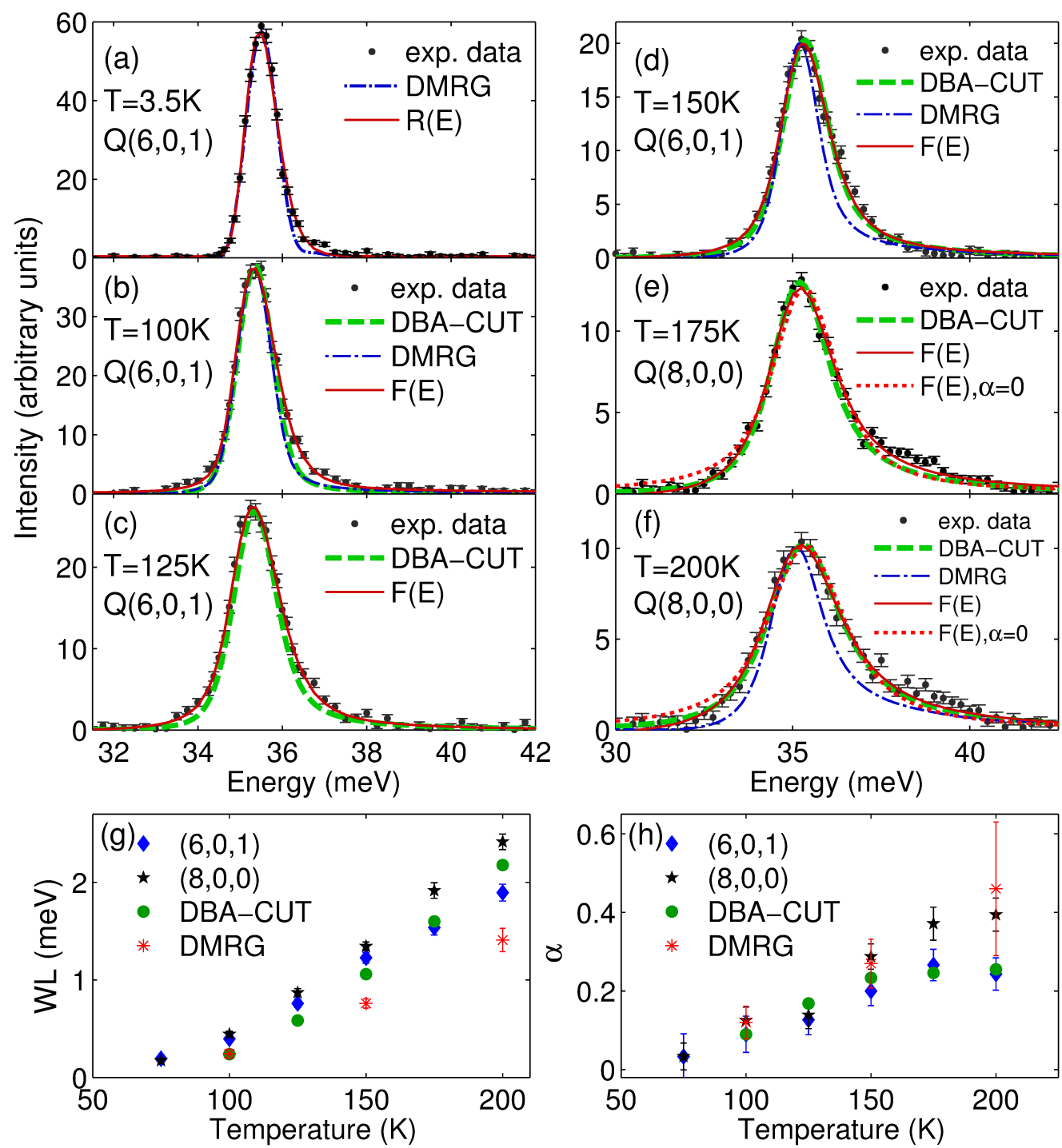

Figure 7.5: (Taken from Ref. $\left[\mathrm{KTF}^{+} 16\right]$.) The figure shows a comparison of the DMRG results computed in this thesis to experimental data and DBA-CUT calculations, both obtained by collaborators. The background-subtracted constant wave-vector INS scans at $(6,0,1)$ and $(8,0,0)$ were measured at (a) $T=3.5 \mathrm{~K}$, (b) $T=100 \mathrm{~K}$, (c) $T=125 \mathrm{~K}$, (d) $T=150 \mathrm{~K}$, (e) $T=175 \mathrm{~K}$, and (f) $T=200 \mathrm{~K}$. At $T=3.5 \mathrm{~K}$ in panel (a), the instrumental resolution function is determined by a fit (solid line) using the asymmetric Gaussian function $G_{\gamma}(E)$ in Eq. (7.6). In panels (b)-(f), the solid lines are the fits of the function $F_{W_{L}, \alpha}(E)$ in Eq. (7.10) to the experimental results in order to extract the Lorentzian width $W_{L}$ and the Lorentzian asymmetry parameter $\alpha$. The dotted lines in panels (e) and (f) represent fits with $\alpha=0$ showing that a symmetric Lorentzian lineshape is not compatible with the experiment. The dash-dotted lines in panels (b), (d), and (f) are the finite-temperature DMRG results for the AFM-FM model obtained in this thesis. Corresponding DBA-CUT results are given by the dashed lines. Panels (g) and (h) show the temperature dependence of the parameters for the Lorentzian width $W_{L}$ and the asymmetry $\alpha$ obtained by fits of Eq. (7.10) to both the experimental and theoretical results. 
In order to finally illustrate the departure from a symmetric Lorentzian lineshape at elevated temperatures, the experimental resolution function $R(E)$ is convolved with an ordinary Lorentzian function

$$
L(E)=\frac{1}{\pi} \frac{\frac{W_{L}}{2}}{\left(E-E_{\text {peak }}\right)^{2}+\left(\frac{W_{L}}{2}\right)^{2}}
$$

modeling only symmetric temperature broadening. Then the resulting fit function is of the form

$$
F(E)=A \cdot L(E) * R(E),
$$

where the prefactor $A$ denotes the peak height and $*$ signifies the convolution. Now $F(E)$ is fitted to the lineshapes at $175 \mathrm{~K}$ and $200 \mathrm{~K}$ in Figs. 7.5(e)-(f) (dotted red line). From this, it becomes clear that the lineshape at these elevated temperatures does not have a symmetric Lorentzian profile.

Next, one would like to parametrize the observed asymmetry in order to study its temperature dependence. A tractable route is to modify the ordinary Lorentzian width $W_{L}$ in Eq. (7.7) by the introduction of a Lorentzian asymmetry parameter $\alpha$ :

$$
L_{W_{L}, \alpha}(E)=\frac{1}{\pi} \frac{\frac{W_{L}+\alpha\left(E-E_{\text {peak }}\right)}{2}}{\left(E-E_{\text {peak }}\right)^{2}+\left(\frac{W_{L}+\alpha\left(E-E_{\text {peak }}\right)}{2}\right)^{2}} .
$$

The parameter $\alpha$ makes the lineshape asymmetric with respect to the peak position $E_{\text {peak }}$. The fitting function $F_{W_{L}, \alpha}(E)$ used for a parametrization of the asymmetric lineshape is thus given as the convolution of an asymmetric Lorentzian $L_{W_{L}, \alpha}(E)$ and the experimental resolution function $R(E)$ :

$$
F_{W_{L}, \alpha}(E)=A \cdot L_{W_{L}, \alpha}(E) * R(E) .
$$

The solid red lines in Figs.7.5(b)-(f) represent the best fits of $F_{W_{L}, \alpha}(E)$ to the experimental data at either $(6,0,1)$ or $(8,0,0)$. The fit results for the width $W_{L}$ and the asymmetry parameter $\alpha$ are depicted as a function of temperature in Figs. 7.5 $(\mathrm{g})-(\mathrm{h})$. The temperature dependence of $W_{L}$ shows that the FWHM of the excitations increases with temperature, which clearly reveals thermal broadening of the excitations. Furthermore, their lineshape is asymmetric above $100 \mathrm{~K}$ and becomes even more asymmetric with rising temperature as $\alpha$ increases in Fig. 7.5(h).

\section{DMRG results}

As motivated in the introduction of this chapter, one main objective of this thesis is to verify the experimentally observed asymmetric thermal lineshape broadening by reliable and accurate DMRG computations for the alternating AFM-FM screw-chain model. The newly developed finite-temperature approach working directly in the frequency domain in combination with linear prediction is employed for this purpose. The technical details that are relevant for the calculation of the dynamical spin structure factor at $T>0$ have been discussed above. The corresponding DMRG results are obtained at $100 \mathrm{~K}$, $150 \mathrm{~K}$, and $200 \mathrm{~K}$ for the alternating AFM-FM chain with dimensionless coupling constants $J_{\text {intra }}=1$ and $J_{\text {inter }}=-0.29$, see Tab. 7.1. The data are rescaled with the slightly 


\begin{tabular}{c|c}
\hline \hline Temperature $(\mathrm{K})$ & $J_{1}(T)(\mathrm{meV})$ \\
\hline 3.5 & $40.92 \pm 0.03$ \\
\hline 100 & $40.60 \pm 0.05$ \\
\hline 150 & $40.18 \pm 0.05$ \\
\hline 200 & $39.69 \pm 0.10$ \\
\hline \hline
\end{tabular}

Table 7.2: Temperature dependence of the interdimer coupling $J_{1}$. The corrections included in $J_{1}(T)$ are smaller than $5 \%$ compared to the base-temperature value of $J_{1}$.

temperature-depend intradimer coupling $J_{1}(T)$ specified in Tab. 7.2. The analysis yielding these values is based on a comparison between DMRG and experimental results and is shown in Appendix B.3. A slight temperature dependence of $J_{1}$ is plausible since for instance lattice distortion may occur in $\mathrm{BaCu}_{2} \mathrm{~V}_{2} \mathrm{O}_{8}$ due to the wide temperature range considered here. The DMRG results are shown as the dash-dotted blue curves in Figs. 7.5(b), 7.5(d), and 7.5(f). Over this broad temperature range, there is good quantitative agreement between the lineshapes calculated by DMRG and probed in the experiment. The resolution of the DMRG-based Chebyshev expansions is enhanced by linear prediction to exactly fit the experimental resolution broadening of $W_{G}=0.86 \mathrm{meV}$ determined above, whereas it does not take account of the slight asymmetry of the experimental resolution in Eq. (7.6). The asymmetric lineshape of the calculations is solely attributed to thermal broadening. Note that the Gaussian broadening $\eta$ introduced in the Chebyshev expansions (see Sec. 3.5.4) is related to $W_{G}$ by $\eta=W_{G} /(2 \sqrt{2 \ln (2)})$ for a vanishing Gaussian asymmetry $(\gamma=0)$ in Eq. (7.6).

In Figs. 7.5(b), 7.5(d), and 7.5(f), the DMRG computations confirm the asymmetric lineshape broadening weighted towards the center of the band. There is good quantitative agreement with the experimental results. The previously explained fit analysis based on Eq. (7.10) is also applied to these DMRG data. The extracted fit parameters for the Lorentzian width $W_{L}$ and the Lorentzian asymmetry $\alpha$ are also included in Figs. 7.5(g)(h). They show good agreement with the experimental values. The calculations thus confirm persistence of correlation effects at elevated temperatures.

\section{Comparison to the diagrammatic approach}

Now the finite-temperature results obtained by the diagrammatic Brückner approach (DBA-CUT) are compared to the DMRG calculations as well as the INS measurements. The DBA-CUT results obtained by Benedikt Fauseweh are displayed as the dashed lines in Fig. 7.5 and also support the asymmetric lineshape broadening. The fit analysis using Eq. (7.10) is also applied to the DBA-CUT data. The resulting fit parameters $W_{L}$ and $\alpha$ are shown in Figs. 7.5(g)-(h). In particular, the analysis reveals a slightly better agreement between the Lorentzian width $W_{L}$ extracted from the DBA-CUT spectra and the experiment than for the DMRG results. However, the peak positions of the DBA-CUT curves display an offset with respect to the maxima of both DMRG and the INS. Therefore, the DBA-CUT data have been shifted for better comparability in Fig. 7.5. While both theoretical approaches quantitatively predict the asymmetric profile observed in INS, DBA-CUT better resolves the lineshape whereas the peak positions are better described by DMRG. The DBA-CUT deviations for the peak position are discussed further below. 


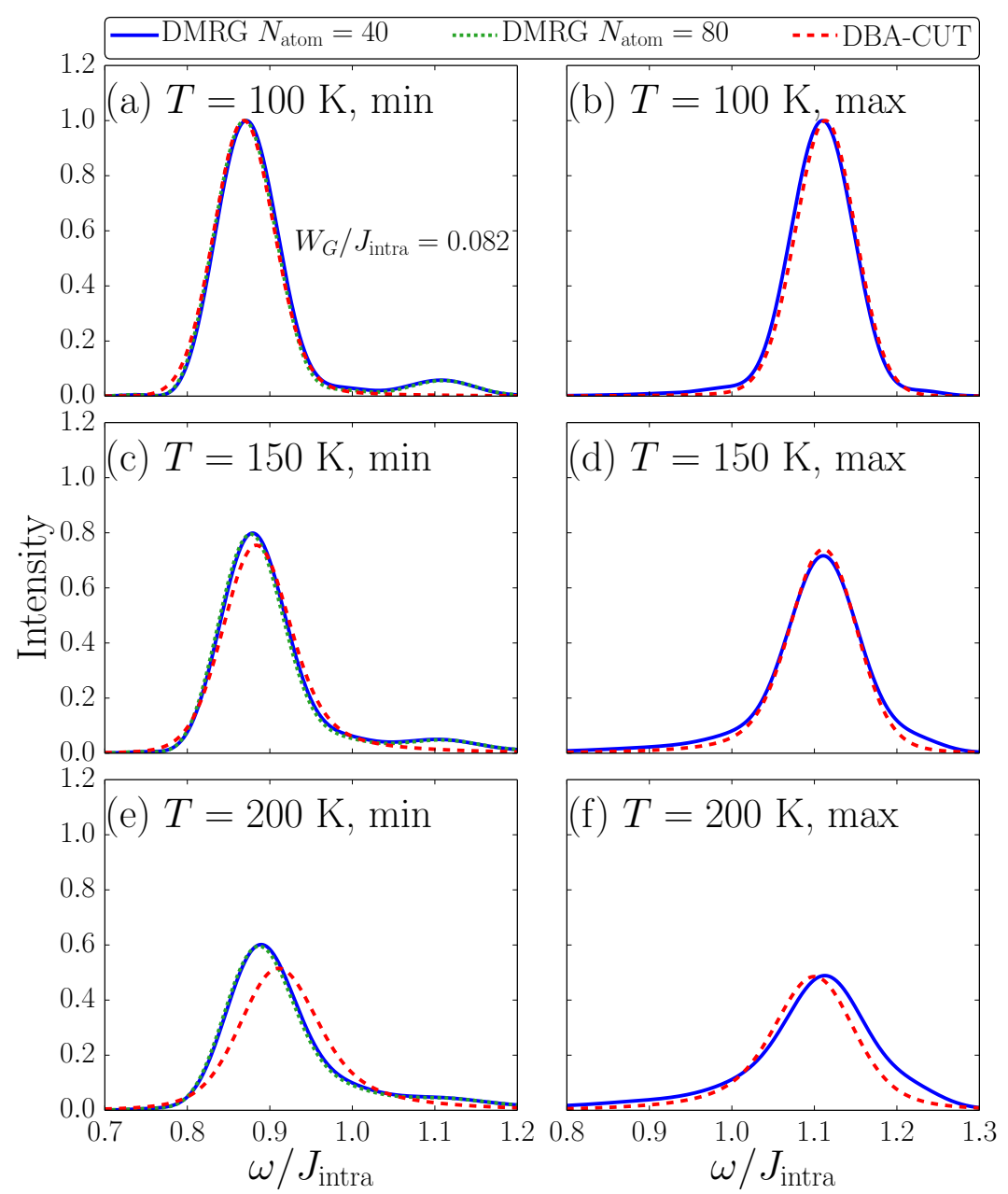

Figure 7.6: (Taken from the supplemental material of Ref. [KTF $\left.\left.{ }^{+} 16\right].\right)$ Comparison of finitetemperature results obtained by the diagrammatic Brückner approach (DBA-CUT) and DMRG (left column) at the minimum and (right column) at the maximum of the single-triplon dispersion for $J_{\text {intra }} / J_{\text {inter }}=-0.29$. Here $J_{\text {intra }}=40.92 \mathrm{meV}$ is used to determine the temperatures in all panels. The DBA-CUT results were calculated by Benedikt Fauseweh, while the DMRG results were obtained in this thesis.

Next, it is interesting to further compare the results of both theoretical approaches in Fig. 7.6. It shows the theoretical data at both the minimum and maximum of the single-triplon dispersion for a lower resolution $\left(W_{G} / J_{\text {intra }}=0.082\right)$ than in Fig. 7.5. At the resolution the results are compared to each other, the DMRG data do not have to be extrapolated by a linear prediction in the Chebyshev moments. At the lowest temperature, $T=100 \mathrm{~K}$, in Fig. 7.6(a)-(b), the peak heights are fixed to one. These scaling parameters are also kept fixed at higher temperatures allowing for a direct comparison of the theoretical approaches in each column of Fig. 7.6. Concerning the evolution of the lineshape with temperature, there is very good agreement of the two approaches up to $T=150 \mathrm{~K}$. At $T=200 \mathrm{~K}$, there is a deviation in the peak positions, which can be explained by the low-temperature approximation inherent to the diagrammatic Brückner approach. While effects already described by the leading order $\exp \left(-\Delta /\left(k_{\mathrm{B}} T\right)\right)$ are accounted for exactly, it could be shown that the shift is an effect $\sim \exp \left(-2 \Delta /\left(k_{\mathrm{B}} T\right)\right)$ [FSU14]. Therefore, deviations from the peak positions with increasing temperature are not unexpected. 
The left column of Fig. 7.6 also shows DMRG results for different system sizes $N_{\text {atom }}=40$ and 80. Since these two DMRG curves are very close to each other, finite-size effects seem to be negligibly small. Moreover, the lineshape of the dominant peak is not significantly altered by adopting a 1D Fourier transform (not shown), which does not take account of the real atom positions. Such a transform is used in the Brückner approach.

In summary, at the resolution shown in Fig. 7.6 both theories show an excellent agreement for the shape, width, and temperature-dependence of the height and good agreement on the position at low temperatures. Deviations between both approaches only occur for very high resolutions as required for the quantitative analysis of the experiment. This leads to the slightly different results for width and asymmetry displayed in Fig. 7.5(g)-(h).

\section{Comparison of the AFM-FM and AFM-AFM models}

Furthermore, a comparison of the thermal lineshape broadening for the AFM-FM and AFM-AFM models is interesting since the effect of the interdimer exchange coupling can be studied in this manner. Therefore, the spectral function at the dispersion minimum is computed by DMRG for both the AFM-FM and AFM-AFM model at $T=100 \mathrm{~K}$. Together with the experimental data for $\mathrm{BaCu}_{2} \mathrm{~V}_{2} \mathrm{O}_{8}$ these results are presented in Fig. 7.7. Both calculations yield an almost identical asymmetric lineshape revealing that there is only a marginal dependence on the sign of the weak interdimer coupling at $T=100 \mathrm{~K}$.

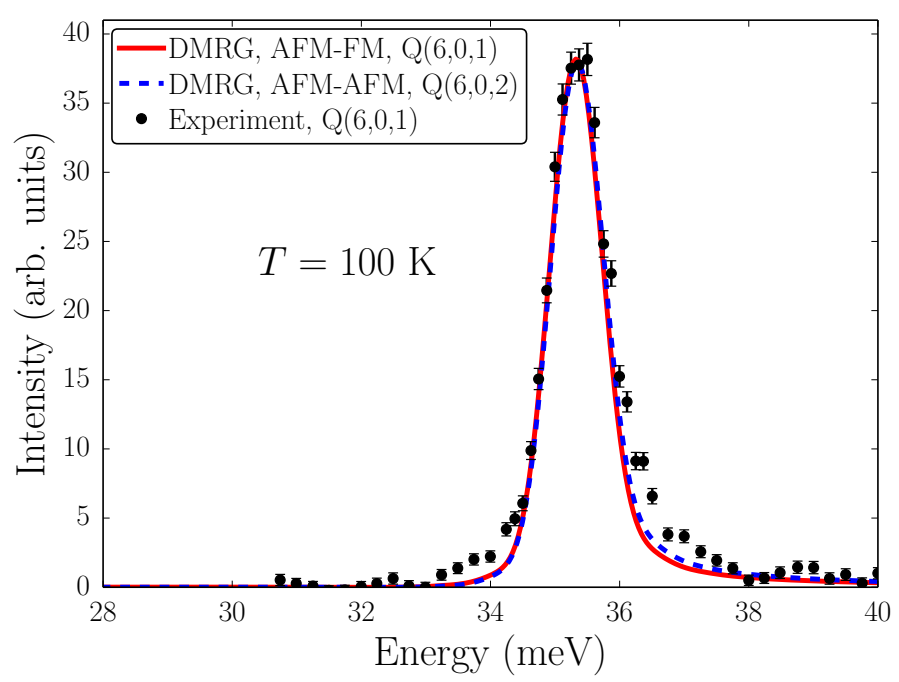

Figure 7.7: (Adapted from the supplemental material of Ref. $\left[\mathrm{KTF}^{+} 16\right]$.) DMRG calculations for the spectral function at the dispersion minimum for both the AFM-FM $[$ at $Q=(6,0,1)]$ and AFM-AFM [at $Q=(6,0,2)]$ model at $T=100 \mathrm{~K}$ compared to the background-subtracted INS data for $\mathrm{BaCu}_{2} \mathrm{~V}_{2} \mathrm{O}_{8}$ at $Q=(6,0,1)$. The asymmetric lineshape broadening does only weakly depend on the sign of the interdimer coupling at $T=100 \mathrm{~K}$. 


\section{Finite-temperature correlation functions}

Furthermore, it is also interesting to study the temperature dependence of spin-spin correlation functions which are readily accessible in the course of an imaginary-time evolution to obtain thermal states. Retaining an internal MPS bond dimension of $m=150$, the calculation was performed using the TDVP method for chains of $L=30$ and 50 sites. Results for both the correlation functions at the strongly-coupled intradimer bonds $\left\langle\mathbf{S}_{i, 1} \mathbf{S}_{i, 2}\right\rangle$ and the weakly-coupled interdimer bonds $\left\langle\mathbf{S}_{i, 2} \mathbf{S}_{i+1,1}\right\rangle$ are shown for $J_{2} / J_{1}=-0.29$ in Fig. 7.8. The intradimer correlations $\left\langle\mathbf{S}_{i, 1} \mathbf{S}_{i, 2}\right\rangle$ in Fig.7.8(a) only show minor deviations from the isolated dimer limit in which the correlations can be calculated exactly as (see Appendix B.2 for the derivation)

$$
\left\langle\mathbf{S}_{1} \mathbf{S}_{2}\right\rangle_{\text {isolated }}=-\frac{3}{4} \frac{1-\mathrm{e}^{-J_{\text {intra }} /\left(k_{\mathrm{B}} T\right)}}{1+3 e^{-J_{\text {intra }} /\left(k_{\mathrm{B}} T\right)}}
$$
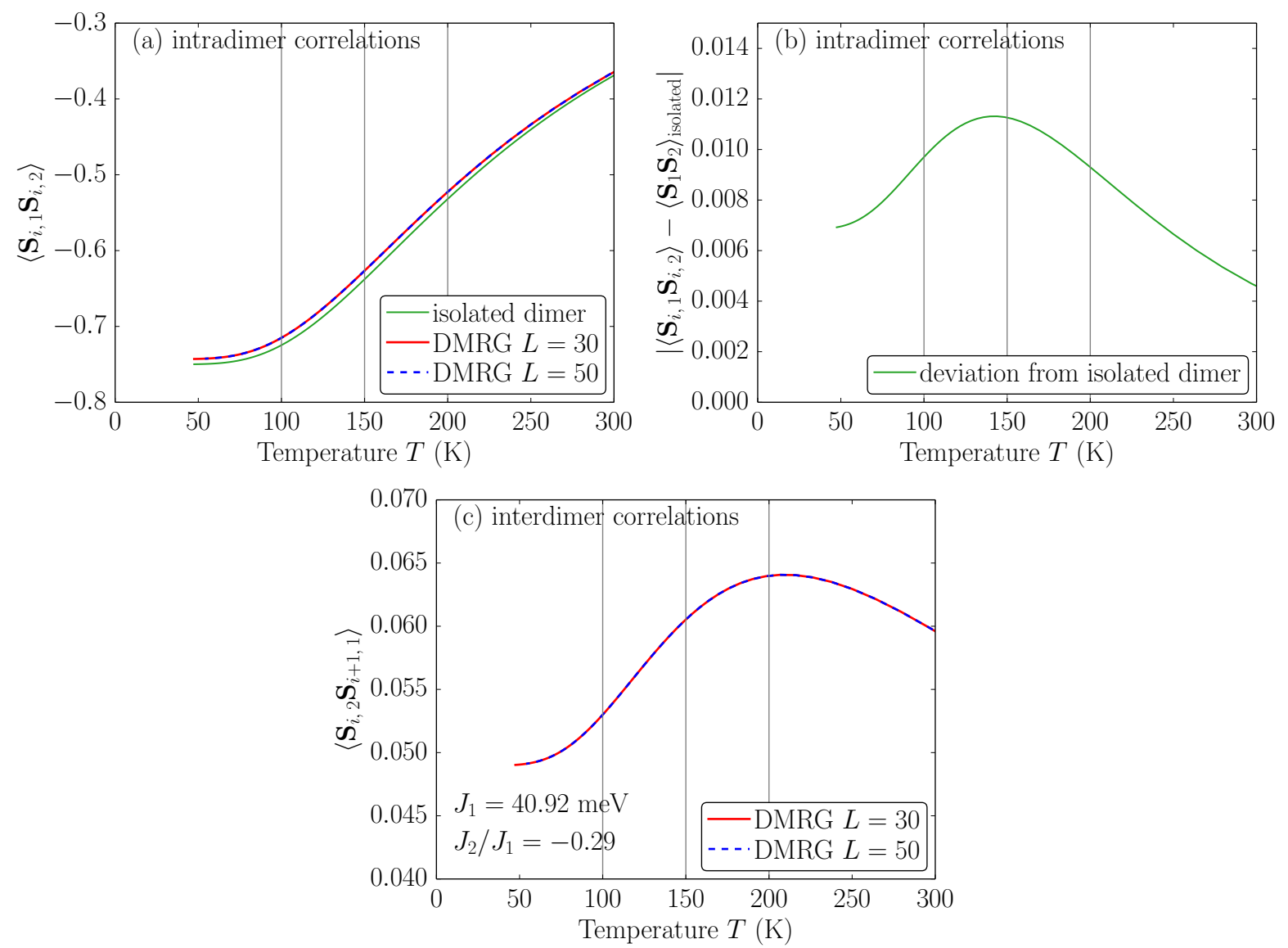

Figure 7.8: DMRG results for the temperature dependence of the spin-spin correlation functions in the AFM-FM model $\left(J_{2} / J_{1}=-0.29\right)$ : (a) Intradimer correlation function $\left\langle\mathbf{S}_{i, 1} \mathbf{S}_{i, 2}\right\rangle$ at the center of a chain with $L=50(L=30)$ sites compared to exact results for an isolated dimer in Eq. (7.11). (b) Deviation $\left|\left\langle\mathbf{S}_{i, 1} \mathbf{S}_{i, 2}\right\rangle-\left\langle\mathbf{S}_{1} \mathbf{S}_{2}\right\rangle_{\text {isolated }}\right|$ between the isolated dimer case and the DMRG calculations for the intradimer correlations $(L=30)$. (c) Interdimer correlation function $\left\langle\mathbf{S}_{i, 2} \mathbf{S}_{i+1,1}\right\rangle$. For a better comparison to the other results for $\mathrm{BaCu}_{2} \mathrm{~V}_{2} \mathrm{O}_{8}$ the temperature axis is rescaled using $J_{1}=40.92 \mathrm{meV}$ in all panels. The vertical lines mark $T=100 \mathrm{~K}, 150 \mathrm{~K}$, and $200 \mathrm{~K}$. 
At first sight, the qualitative behavior is very similar in Fig.7.8(a). To have a closer look, the temperature dependence of the deviation between the DMRG results for weakly coupled dimers $\left(J_{2} / J_{1}=-0.29\right)$ and a single dimer is plotted in Fig. 7.8(b). The difference shows a maximum slightly below $T=150 \mathrm{~K}$ before the isolated dimer curve is monotonically approached towards higher temperatures. In the limiting case of $T=\infty$ the correlations are zero. Interestingly, the maximum falls into the range of temperatures where the asymmetric lineshape is observed for $\mathrm{BaCu}_{2} \mathrm{~V}_{2} \mathrm{O}_{8}$.

The temperature dependence of the interdimer correlation function $\left\langle\mathbf{S}_{i, 2} \mathbf{S}_{i+1,1}\right\rangle$ is depicted in Fig. 7.8(c). It represents the deviation from the isolated dimer limit since the correlations between different dimers are zero in this case. Again, in the temperature range where the asymmetric lineshape is observed for $\mathrm{BaCu}_{2} \mathrm{~V}_{2} \mathrm{O}_{8}$, there is an increase of the correlations between two adjacent dimers which are assuming a maximum slightly above $T=200 \mathrm{~K}$.

Although the studied effects in both intradimer and interdimer correlation functions in Figs. 7.8(b)-(c) are very tiny, one may wonder whether this increase in quantum correlations at elevated temperatures is related to the increase of the Lorentzian asymmetry parameter $\alpha$ found in the same temperature range in Fig. 7.5(h). This question will be discussed as a perspective for future research in Sec. 7.5 below. 


\section{Intraband excitations $\mid 7.4 .4$}

INS data for the compound copper nitrate showed a continuum of temperature-induced excitations at small energies which could be attributed to scattering within the one-triplon band $\left[\mathrm{TLJ}^{+} 12\right]$. These excitations are in agreement with full diagonalization results for small systems of $L=16$ sites [ML06, Luc08] and an analytical approach based on a low-temperature expansion [JEK08].

Since $\mathrm{BaCu}_{2} \mathrm{~V}_{2} \mathrm{O}_{8}$ is described by an AFM-FM model instead of the AFM-AFM model for copper nitrate, the presence of such intraband transitions in $\mathrm{BaCu}_{2} \mathrm{~V}_{2} \mathrm{O}_{8}$ is an interesting question. However, INS measurements of the intraband continuum in $\mathrm{BaCu}_{2} \mathrm{~V}_{2} \mathrm{O}_{8}$ turned out to be challenging due to its low intensity [Kly16]. Therefore, only theoretical predictions for the temperature dependence of these excitations is presented here. To this end, the finite-temperature frequency-domain approach developed as a main part of this thesis (see Sec. 3.6) is used. In order to study the corresponding momentum dependence, MPS-based Chebyshev expansions with respect to the Liouville operator are obtained by the computation scheme in Eq. (7.5c). The calculations are thus performed in real arithmetics and without linear prediction, using an internal MPS bond dimension of $m=200$. The DMRG results for the dynamical spin structure factor $S_{z z}(Q, \omega)$ along the $(6,0, \mathrm{~L})$ direction are presented in Fig. 7.9. The finite-temperature data obtained for a chain of $L=30$ sites clearly shows the appearance of continua at small energies. With rising temperature, their intensities increase further. However, also note that Figure 7.9 shows the intensities on a logarithmic scale. It turns out that the intensity of the thermallyactivated intraband transitions is only a small fraction of the weight associated with the one-triplon excitations. This explains why INS measurements of these excitations are not straightforward.

There is an analogous observation of low-frequency intensity for AFM spin chains. This is oftentimes referred to as Villain mode which was first reported for Ising antiferromagnets [Vil75]. In contrast to the model for $\mathrm{BaCu}_{2} \mathrm{~V}_{2} \mathrm{O}_{8}$, here the excitations are domain walls in the AFM order and not triplons. 

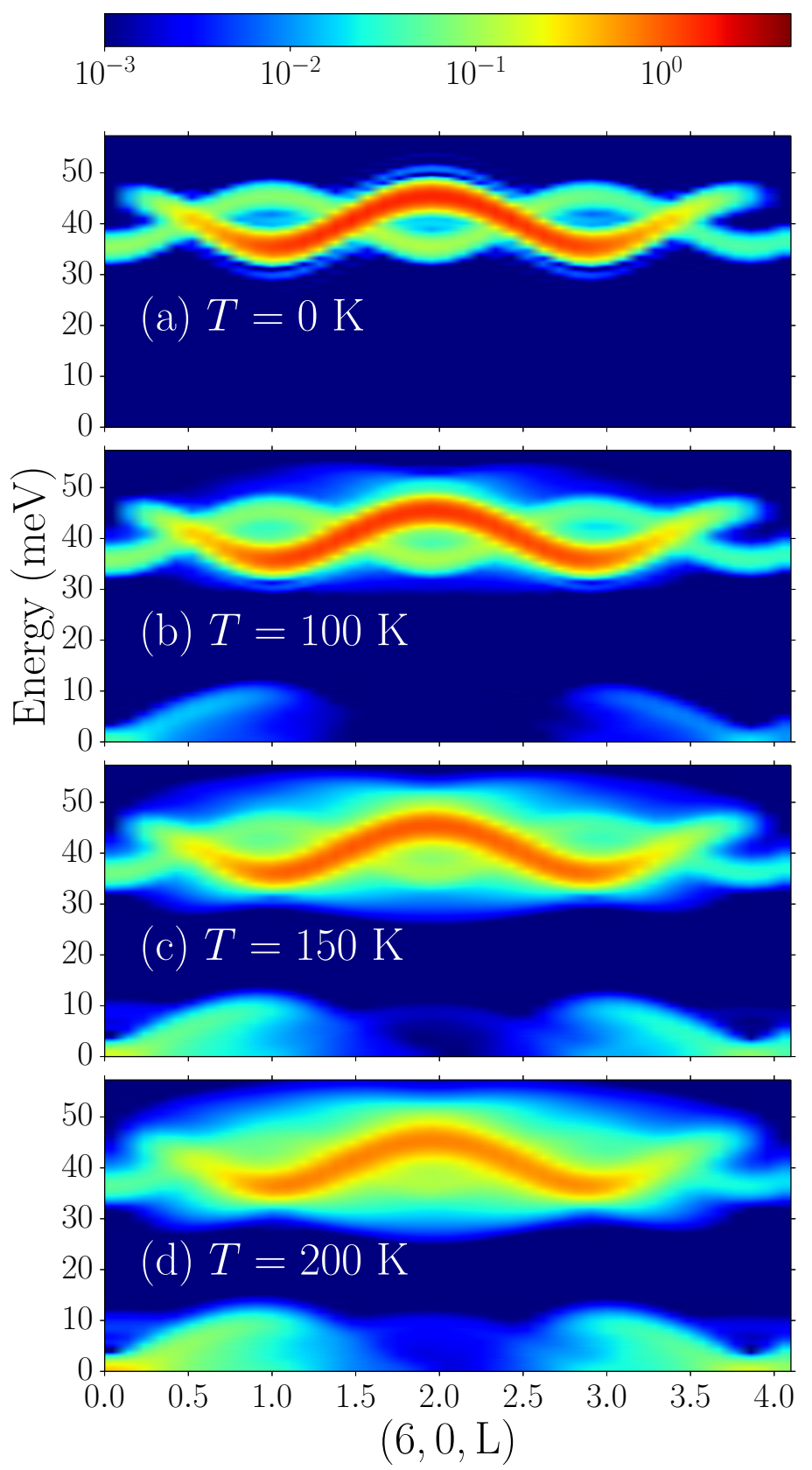

Figure 7.9: DMRG results for the dynamical spin structure factor $S_{z z}(Q, \omega)$ showing the appearance of intraband excitations at small energies and the thermal broadening of the onetriplon dispersion along the $(6,0, \mathrm{~L})$ direction at temperatures of (a) $T=0 \mathrm{~K}$, (b) $100 \mathrm{~K}$, (c) $150 \mathrm{~K}$, and (d) $200 \mathrm{~K}$. The finite-temperature results are obtained for $L=30$ whereas a system of $L=40$ sites is studied at $T=0$ in panel (a). The temperatures and the energy axis is scaled using a fixed value of $J_{1}=40.92 \mathrm{meV}$ for simplicity. The broadening is $\eta=0.035$ (corresponding to $W_{G} \approx 3.37 \mathrm{meV}$ ) and therefore higher than in the INS experiments. 


\section{\begin{tabular}{l|l} 
Conclusion & 7.5
\end{tabular}}

One result of this chapter is the identification of the Hamiltonian for the quasi-1D dimer compound $\mathrm{BaCu}_{2} \mathrm{~V}_{2} \mathrm{O}_{8}$. It is shown that $\mathrm{BaCu}_{2} \mathrm{~V}_{2} \mathrm{O}_{8}$ is described by a spin- $1 / 2$ alternating Heisenberg chain. The weaker interdimer coupling is FM whereas the stronger intradimer coupling is AFM. This corrects the long-held view from all previous experimental [HKI04, HTI06, GPB ${ }^{+}$55, LX05, SMD08] and theoretical [KW06, SMD08] works that the interdimer coupling $J_{2}$ is AFM or negligibly small. However, the exchange paths $J_{1}$ and $J_{2}$ predicted by electronic structure calculations [KW06, SMD08] are compatible with our results. Moreover, an FM interdimer exchange $J_{2}$ can also be reconciled with a previous prediction for the bridge angle of the corresponding superexchange path $(\mathrm{Cu}-\mathrm{O}-\mathrm{Cu})$. The predicted value is $94^{\circ}$ [KW06] and therefore very close to the crossover from AFM to FM according to the Goodenough-Kanamori-Anderson rules [And50, Kan59, Goo60].

Apart from the identification of the Hamiltonian, the DMRG calculations taking into account the atom positions in Fig. 7.3 also verified that the weaker dispersion in the INS spectra appears due to the screw-chain geometry of $\mathrm{BaCu}_{2} \mathrm{~V}_{2} \mathrm{O}_{8}$.

The main result of this chapter is that there is an extraordinary coherence of magnetic excitations at elevated temperatures in the gapped dimer compound $\mathrm{BaCu}_{2} \mathrm{~V}_{2} \mathrm{O}_{8}$. Most importantly, the temperature effects in $\mathrm{BaCu}_{2} \mathrm{~V}_{2} \mathrm{O}_{8}$ are thus accounted for by the AFMFM model. This is found by calculating the temperature dependence of the dynamical spin structure factor directly in the frequency domain by MPS-based Chebyshev expansions for this model and comparing them to high-resolution INS measurements. The computed lineshapes quantitatively describe the experimentally observed lineshapes in $\mathrm{BaCu}_{2} \mathrm{~V}_{2} \mathrm{O}_{8}$ over a broad temperature range. Fitting analyses of both the computed and experimentally probed lineshapes, reveal an asymmetric broadening at the minimum of the one-triplon dispersion which increases with temperature. The fit results for the experimental and theoretical asymmetry parameters are in good quantitative agreement.

In order to further support these results, the asymmetric lineshape broadening is also studied by the recently developed theoretical approach DBA-CUT [FSU14, FU15]. As shown in Fig. 7.5, there is good quantitative agreement between the experimentally observed and the theoretically predicted lineshapes obtained by both DBA-CUT and DMRG.

Furthermore, a comparison to the quasi-1D compound copper nitrate, which can be described by a AFM-AFM dimer model and also exhibits asymmetric lineshape broadening $\left[\mathrm{TLJ}^{+} 12\right]$, leads directly to the next conclusion. The persistence of strong correlations in dimerized quantum magnets is independent of the sign of the interdimer exchange coupling. In particular, DMRG calculations at $T=100 \mathrm{~K}$ even showed that the asymmetry in the lineshape is almost identical for the AFM-FM and AFM-AFM models.

In this context, it has to be pointed out that the theoretical results presented here represent an improvement over previous results obtained by full diagonalization [ML06, Luc08] and the field theoretical treatment [JEK08] of an AFM-AFM dimer model for copper nitrate $\left[\mathrm{TLJ}^{+} 12\right]$. Finite-temperature Chebyshev expansions formulated in Liouville space give accurate results for the dynamical spin structure factor for significantly larger sys- 
tems of up to $N_{\text {atom }}=80$ atoms, which is an important achievement of this thesis. These computations also include the details of the crystal structure.

Last but not least, DMRG results for the temperature dependence of interdimer and intradimer correlation functions are presented. In this study the deviations from the isolated-dimer limit are studied and turn out to be rather tiny. However, these slight deviations are most pronounced over the temperature range in which the asymmetric lineshape broadening increases for $\mathrm{BaCu}_{2} \mathrm{~V}_{2} \mathrm{O}_{8}$. A possible relation between the asymmetry and these quantum fluctuations needs further studies and would be an interesting question for future research. Here also a connection to the quantum mechanical entanglement of the system is of interest. However, the quantification of finite-temperature entanglement itself poses a challenging problem as no commonly accepted measure exists [AFOV08, ACL12]. 



\section{Summary and conclusion

One key result of this thesis is the development of an efficient and unbiased numerical approach for the calculation of finite-temperature spectral functions of 1D strongly correlated quantum systems. The approach is implemented in the framework of MPS and works directly in the frequency domain.

Another main achievement is that the developed method could be successfully applied to two different spin-1/2 models for the quasi-1D compounds copper pyrimidine dinitrate $(\mathrm{Cu}-\mathrm{PM})$ and $\mathrm{BaCu}_{2} \mathrm{~V}_{2} \mathrm{O}_{8}$, allowing for a direct comparison to high-resolution experimental results for dynamical quantities at $T>0$. A summary and concluding remarks concerning these projects are given below. First, methodical aspects as well as their implications for future research are discussed.

\section{MPS formulation of frequency-space dynamics at finite temperature}

A main component of the developed finite-temperature MPS approach is the established purification $\left|\Psi_{T}\right\rangle$ of the thermal density matrix which is employed since MPS can only represent pure quantum states. In order to arrive at such a pure-state wave function describing a generally mixed finite-temperature state, the system's degrees of freedom need to be doubled [UHT82]. As a consequence, one works with a tensor product space $\mathcal{H}_{P} \otimes \mathcal{H}_{Q}$ of the physical and an auxiliary state space. Choosing a Liouvillian formulation for the frequency-space dynamics of the purification $\left|\Psi_{T}\right\rangle$, it is possible to extend existing zerotemperature frequency-domain methods to the calculation of spectral functions at $T>0$. Proof-of-principle calculations for two such approaches, both MPS-based Chebyshev and continued fraction expansions (CFE) of dynamical correlation functions, are presented in Chapter 5. They reveal that the Liouvillian formulation of finite-temperature frequencyspace dynamics allows for an efficient as well as unbiased treatment of 1D quantum spin chains in the framework of MPS. This methodical insight is one main result of this thesis. Both methods give accurate results for the finite-temperature dynamical spin structure factor of the 1D $X X$ model, which is verified by a comparison to exact results. The temperature dependence of the same quantity is also calculated for an antiferromagnetic Heisenberg chain in a uniform and a staggered magnetic field, serving as a further nontrivial test case. From the calculations, it is concluded that the Chebyshev MPS method is more efficient than the CFE at $T>0$. It therefore better resolves the dynamical structure factor for these models at a much smaller internal MPS bond dimension.

It is useful to regard the method developed in this thesis in relation to other established methods for the calculation of finite-temperature spectral functions. For instance, quantum Monte Carlo (QMC) simulations based on the stochastic series expansion scheme [San10] are a state-of-the-art approach. Here spectral functions need to be obtained by a challenging analytic continuation procedure from imaginary-frequency data [JG96, 
Bea04], which can be hard to control in certain cases. On the contrary, the finitetemperature Chebyshev MPS approach provides an approximation which can be well controlled. Reliable error estimates help to detect numerical artifacts. One drawback of the iterative Chebyshev MPS approach is that it cannot be easily parallelized on a large scale such as the stochastic sampling in QMC simulations. Therefore, it is possible to obtain the QMC results in only a small fraction of the time elapsed in the course of the MPS calculations, but the total CPU time used for QMC can be significantly higher.

In this context, one should also comment on existing DMRG approaches for the calculation of spectral functions via real-time evolution of a purification [KBM12, Bar13] followed by a Fourier transform to frequency space. At zero-temperature, the MPS Chebyshev approach has been benchmarked against two different variants of MPS-based time-evolution methods only recently [WJMS15, BWvDG16]. The analysis in Ref. [BWvDG16] concludes that neither the time-domain nor the Chebyshev method shows clear advantages at $T=0$. However, note that comparisons between two numerical methods depend on the details of their implementation as well as the studied models, parameters, and quantities. At finite temperature, there exist no benchmark calculations of this kind. This question is left for future investigations as a meaningful analysis would be a comprehensive project.

Another aspect is that the dynamical response at very low frequency calculated by MPS time evolution using the purification trick can be limited as it depends on the long-time behavior of time-dependent correlation functions [KKM14, KKHM15]. For MPS-based Chebyshev expansions at $T>0$, it can be concluded that the resolution at low frequency is constant and not degrading even at strictly $\omega=0$. For instance, a continuum of intraband transitions could be observed as a low-frequency feature of very weak intensity in the model calculations for $\mathrm{BaCu}_{2} \mathrm{~V}_{2} \mathrm{O}_{8}$, see Fig. 7.9. This is expected to be a general characteristic of finite-temperature frequency-domain approaches formulated in Liouville space and should definitely be further explored. It is an interesting question to assess the full potential, e.g., in the study of transport properties [HMHB07, KKHM15].

\section{Dynamical properties of the sine-Gordon quantum magnet $\mathrm{Cu}-\mathrm{PM}$}

Modeling the Dzyaloshinskii-Moriya interactions in the quasi-1D sine-Gordon compound $\mathrm{Cu}-\mathrm{PM}$ by an effective staggered field, the spectral functions for this system were studied for a broad range of the uniform magnetic field $h_{z}$ applied in the longitudinal direction.

At zero temperature, the frequency-field dependence and the intensities of several elementary excitations such as the soliton and breather excitations were calculated by MPS-based Chebyshev expansions with unprecedented accuracy. The system could therefore be studied beyond the limitations of existing low-energy descriptions by sine-Gordon field theory and at high magnetic fields. The frequency-field dependence of the breathers calculated in this thesis is in good agreement with the field theory. Concerning the frequency-field dependence of the single-soliton resonance, the MPS calculations could resolve the discrepancy between ESR experiments and sine-Gordon field theory and are in excellent agreement with the experiments. The explanation is the band curvature of the singlesoliton dispersion found in the MPS results for the dynamical spin structure factor, see Fig. 6.8. The Lorentz-invariant dispersion assumed for the field theoretical predictions 
cannot account for such effects. Furthermore, the curvature is compatible with recent INS results for the sine-Gordon magnet $\mathrm{KCuGaF}_{6}\left[\mathrm{UTK}^{+} 15\right]$.

Moreover, the presence of boundary as well as composite boundary-bulk excitations was assessed for the effective Hamiltonian modeling $\mathrm{Cu}-\mathrm{PM}$. While such excitations have been predicted by a boundary sine-Gordon field theory at $T=0$ [FO12], their intensity has not been determined. One result is that only the boundary bound state (BBS) directly below the first breather is found in the calculations for finite systems with open boundary conditions. It was shown that its intensity scales to zero in the thermodynamic limit. The predicted composite boundary-bulk resonances have too small intensities to be visible in the calculated spectral functions.

Motivated by experimental hints of strong temperature dependencies of ESR line widths in $\mathrm{Cu}-\mathrm{PM}$ [Zvy12], the temperature dependence of the breather excitations at frequencies $M_{n}$ was studied. Besides the thermal broadening of the breathers, the focus at $T>0$ was on additional thermally activated transitions between the sine-Gordon excitations [FO12]. In particular, the presence of interbreather transitions at $M_{n}-M_{m}$ with small intensities is confirmed by DMRG and exact diagonalization calculations. The B1-B2 interbreather transition was probed in the accompanying ESR experiments and its frequency-field dependence is in excellent agreement with the one expected from both zero-temperature DMRG calculations and the sine-Gordon field theory. Unfortunately, the B1-B2 transition is found directly below the BBS and could therefore not be resolved in the finite-temperature spectral functions calculated by the newly developed MPS method. However, there is evidence for the $M_{3}-M_{2}$ transition in these MPS results. Since the BBS is absent in the ED calculations with periodic boundary conditions contributed by Andreas Honecker, another finding is that several interbreather excitations were observed up to temperatures of about $T / J=0.5$ at intermediate fields of $h_{z} / J=0.6$ and $h_{z} / J=1$.

In addition, a rapid redistribution of spectral intensity with increasing temperature, originally reported in Ref. [IE03], has been revisited in Fig. 6.13. In this thesis, the temperature effect could be identified as the crossover between the single-soliton resonance at low $T$ and the paramagnetic line emerging at $\omega=h_{z}$ towards higher temperatures.

As a perspective for future experimental research, impurity doping in sine-Gordon magnets such as the quasi-1D compound $\mathrm{Yb}_{4} \mathrm{As}_{3}\left[\mathrm{KIM}^{+} 01\right]$ offers a promising experimental realization for studying boundary resonances. This is interesting as the boundary bound state [FO12], which was found in the presented DMRG calculations for $\mathrm{Cu}-\mathrm{PM}$, is not observed in the accompanying ESR experiments.

\section{Coherent quantum behavior at elevated temperatures

It is shown that the magnetic excitations in the compound $\mathrm{BaCu}_{2} \mathrm{~V}_{2} \mathrm{O}_{8}$ can be well described by a spin- $1 / 2$ dimer chain with an antiferromagnetic intradimer coupling $J_{\text {intra }}$ and a weaker ferromagnetic interdimer coupling $J_{\text {inter. }}$. This corrects the long-held view from previous experimental [HKI04, HTI06, $\mathrm{GPB}^{+} 05$, LX05, SMD08] and theoretical [KW06, SMD08] studies that $J_{\text {inter }}$ is antiferromagnetic or negligible. 
A main result of this thesis is that the extraordinary coherence of the magnetic excitations in $\mathrm{BaCu}_{2} \mathrm{~V}_{2} \mathrm{O}_{8}$ at elevated temperatures is confirmed by DMRG calculations. In particular, the finite-temperature method developed in this thesis is used in combination with a linear prediction of Chebyshev moments to compute the dynamical spin structure factor. The calculations reveal that the asymmetric lineshape broadening due to the persistence of strong correlations is an effect which is inherent to the model Hamiltonian.

An increasingly asymmetric profile of the lineshape with rising temperature has been previously observed for dimerized systems, e.g., in a combined experimental and theoretical study $\left[\mathrm{TLJ}^{+} 12\right]$ for the quasi-1D dimer-chain compound copper nitrate, in which both $J_{\text {intra }}$ and $J_{\text {inter }}$ are antiferromagnetic. However, the system sizes accessible before via full diagonalization were rather limited $(L=16)$ and the field theoretical treatment was based on a low-temperature expansion. Therefore, the investigation of a similar system such as $\mathrm{BaCu}_{2} \mathrm{~V}_{2} \mathrm{O}_{8}$ with a method allowing for the treatment of larger systems $(L=40)$ and controlled approximations represents an important improvement. It also led to the conclusion that the persistence of strong correlations in dimerized quantum magnets is independent of the sign of the interdimer exchange coupling.

Most importantly, the DMRG calculations obtained for $\mathrm{BaCu}_{2} \mathrm{~V}_{2} \mathrm{O}_{8}$ are directly compared to the magnetic excitation spectra probed by inelastic neutron scattering at different temperatures, i.e., $T=100 \mathrm{~K}, 150 \mathrm{~K}$, and $200 \mathrm{~K}$. There is good quantitative agreement for the increasingly asymmetric lineshape of the single-triplon excitation. This demonstrates that the quantitative prediction of strongly correlated behavior over a broad temperature range is possible for $\mathrm{BaCu}_{2} \mathrm{~V}_{2} \mathrm{O}_{8}$.

Quantitative predictions of this type can simplify the identification of compounds, in which coherences at elevated temperatures are possible. This is a fundamental issue which is relevant for potential applications in quantum devices [NC00].

The newly developed MPS method could be applied to calculate finite-temperature spectral functions for gapped 1D antiferromagnets with higher spin. For instance, the spin $S=1$ Heisenberg chain also hosts a gapped excitation at zero temperature. Therefore, one objective of future research could be to quantify the asymmetric thermal lineshape broadening in such systems. Furthermore, the method developed in this thesis is well suited for the study of boundary states as revealed in the numerical study of Cu-PM. Thus, it could be applied to investigate signatures of topological boundary states [HK10] in the spin $S=1$ Heisenberg chain.

Moreover, the temperature dependence of the static interdimer and intradimer correlation functions is studied. There are small deviations from the isolated dimer limit. These are however most pronounced in the temperature range in which the asymmetric lineshape broadening is observed for $\mathrm{BaCu}_{2} \mathrm{~V}_{2} \mathrm{O}_{8}$. This raises the question of a possible connection between the asymmetry and such small corrections to the isolated dimer limit. In this respect, the quantum mechanical entanglement in the system may lead to further insights. However, the characterization of entanglement at finite temperature is a very extensive question itself as no commonly accepted measure exists so far [AFOV08, ACL12]. Such issues are interesting for future investigations. 


\section{\begin{tabular}{l|l} 
Further perspectives & 8.4
\end{tabular}}

Having already mentioned several questions for future investigations along the lines of the concluding remarks above, the author would like to conclude with some further comments and ideas:

Here one central aspect which opens a broad range of possibilities for future method development is the flexibility of the Liouvillian formulation. Therefore, it is conceptually possible to use other tensor network states [VCM08] as MPS in combination with the Liouvillespace formulation. An example are projected entangled pair states (PEPS) which are a generalization of MPS to two dimensions. The development of efficient algorithms for the manipulation of such states is a subject of current research $\left[\mathrm{PBT}^{+} 15\right.$, Cor16].

Apart from MPS-based moment expansions of spectral functions such as the Chebyshev expansions employed in this thesis, it remains an open question whether further zero-temperature frequency-domain methods can be implemented using the Liouvillian formulation. MPS-based correction-vector approaches [Jec02, $\left.\mathrm{WVS}^{+} 09\right]$ are interesting candidates as they allow for high-accuracy calculations of spectral functions, with the drawback that each frequency needs to be addressed separately. However, this might not matter too much if one is only interested in low-frequency transport properties at $T>0$.

In this thesis, the MPS code has been developed from scratch and does not exploit symmetries of the Hamiltonian since in Chapters 5 and 6 the Hamiltonian mainly studied in this thesis does for instance not conserve the $z$ component of the total spin. This is the case since the Dzyaloshinskii-Moriya interactions, which are modeled by a small staggered field, break the spin rotational invariance of the isotropic Heisenberg model. However, DMRG or MPS methods [Sch05a, Sch11, Wei12] generally allow for the exploitation of quantum numbers associated with symmetries of a Hamiltonian. As an example, the use of an Abelian U(1) symmetry such as the conservation of the $z$ component of the total spin can lead to a speedup of the calculations by a factor between 10 and 100 . The study of the dimerized spin-1/2 Heisenberg chain in Chapter 7 reveals that it is possible to obtain meaningful results without the exploitation of such a symmetry. However, for future investigations the implementation of $U(1)$ symmetries would be beneficial since a treatment of longer-ranged interactions, ladder systems, or the fermionic Hubbard model involves even more degrees of freedom than in spin- $1 / 2$ systems.

For future method development, it may also be interesting to combine the MPS-based Chebyshev expansions at $T>0$ with other approaches. One candidate is cluster perturbation theory (CPT) [SPPL00, SPP02] which can also be applied to spin systems [OBS10]. This method works with small clusters which are tiled together to an infinite 1D or 2D system by treating the intercluster interactions with perturbation theory. For the calculation of the spectral functions for the full system, the dynamical correlation functions are only calculated on small clusters. This can be achieved by Chebyshev expansions at $T>0$ as developed in this thesis. One crucial modification is that both the real and imaginary parts of the dynamical correlation function are needed [BS14]. 

Appendices 



\section{Further results for $\mathrm{Cu}-\mathrm{PM}$}

The content of the following two sections with additional results for the material $\mathrm{Cu}-\mathrm{PM}$ has been published in the appendix of Ref. $\left[\mathrm{THP}^{+} 16\right]$.

\section{Frequency-field plots $\mid$ A.1}

In this appendix, additional frequency-field plots of the ESR absorption intensity computed by DMRG-based Chebyshev expansions at $T=0$ are provided in Fig. A.1. Note the two different expansion orders $N$ and that consequently the Gaussian broadening included here depends on both $\omega$ and the spectral width $W\left(h_{z}\right)$.

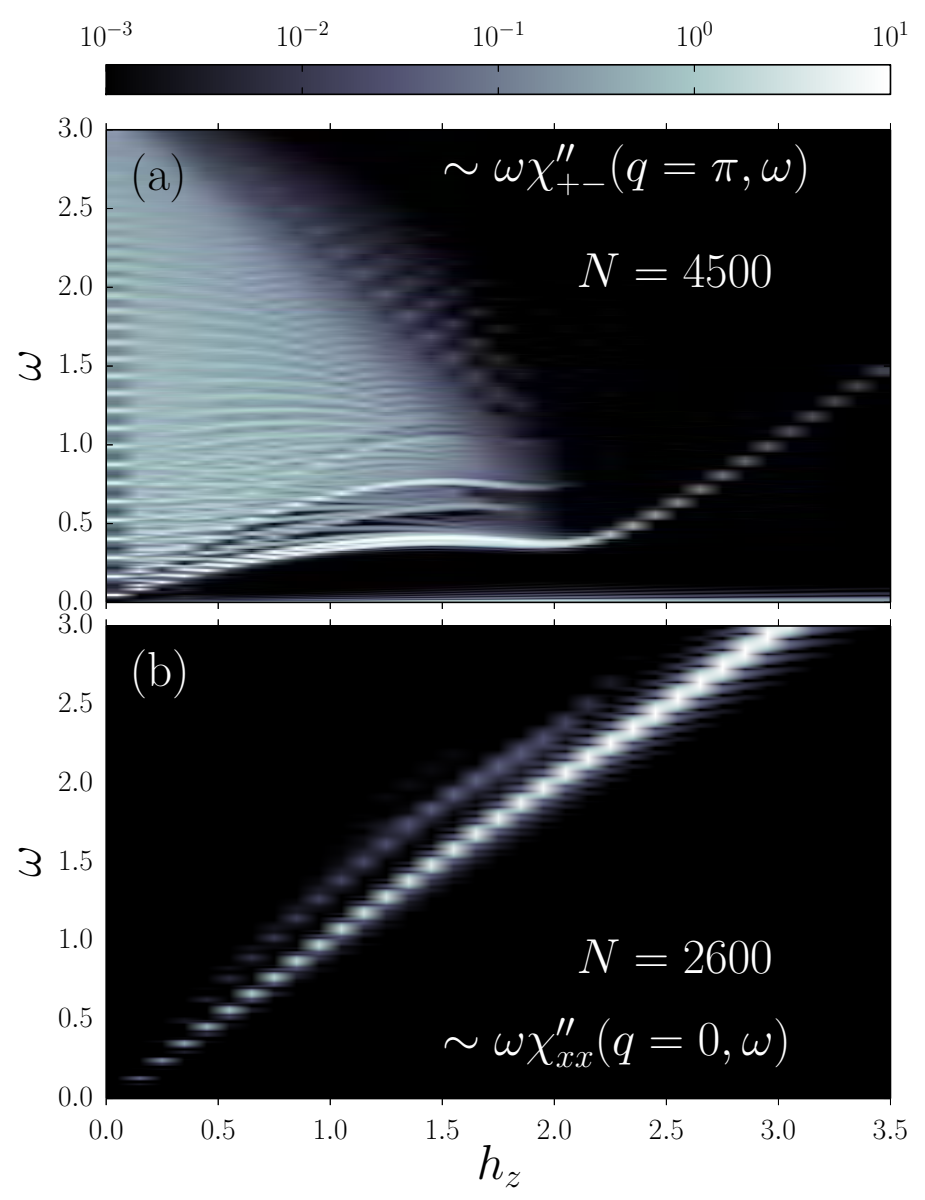

Figure A.1: (Published in Ref. [THP $\left.{ }^{+} 16\right]$.) Frequency-field plots of the $T=0$ absorption intensities $\sim \omega \chi_{+-}^{\prime \prime}(q=\pi, \omega)$ (a) and $\sim \omega \chi_{x x}^{\prime \prime}(q=0, \omega)$ (b) for $L=80$ and $c=0.083$. The results were obtained by DMRG-based Chebyshev expansions for fixed fields $h_{z} \in[0,3.4]$ for a step increment of $\Delta h_{z}=0.1$. 


\section{Finite-size analysis of ED results}

Here a finite-size analysis for the ED data at $h_{z}=1$ shown in Fig. 6.13(b) is presented. To this end, the ESR absorption intensity $I_{x x}(q=0, \omega)$ is plotted for different system sizes at various temperatures in Fig. A.2. Note that at $T / J=0$ the lineshape resembles the thermodynamic limit for all studied system sizes, while at $T / J=0.125$ and $T / J=0.5$ the results for the largest system should be very close to those of an infinite system. Only at $T / J=0.25$, one still observes finite-size effects for $L=20$.
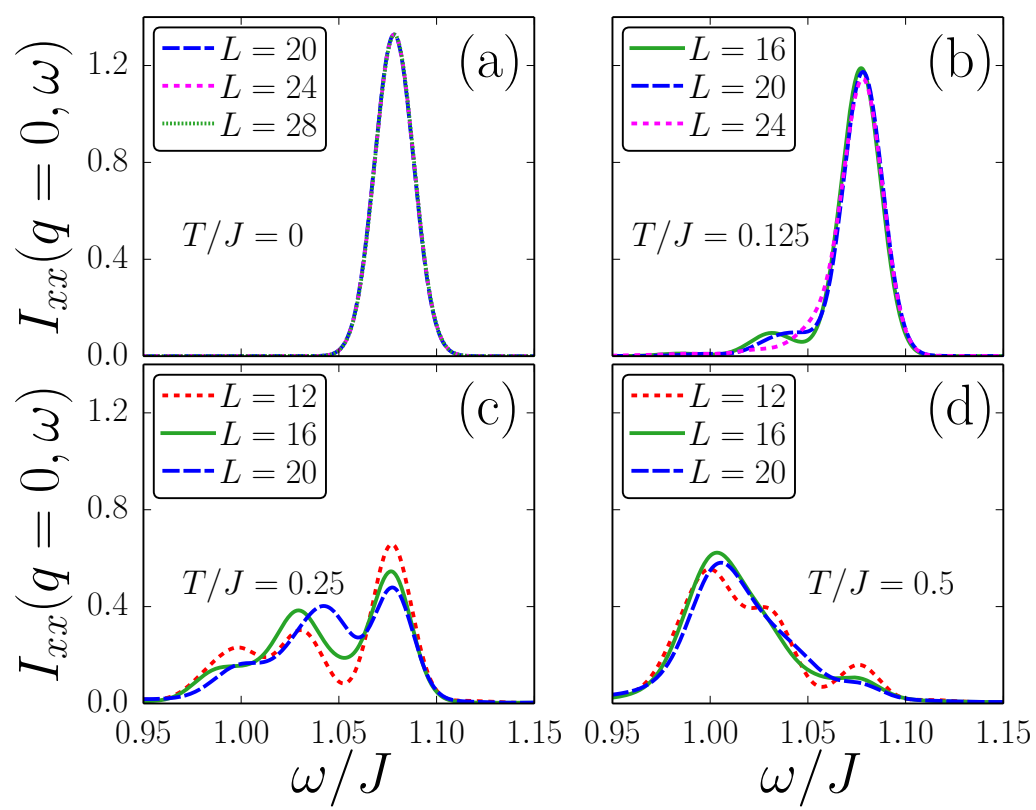

Figure A.2: (Published in Ref. [THP $\left.{ }^{+} 16\right]$.) Finite-size analysis of the ED results with PBCs at $h_{z}=1$ contained in Fig. 6.13(b). 


\section{Further results for $\mathrm{BaCu}_{2} \mathrm{~V}_{2} \mathrm{O}_{8}$}

\section{Dimer susceptibility $\mid$ B.1}

In this appendix the static magnetic susceptibility of weakly coupled dimers in one dimension is derived using a molecular field approximation. To this end, an isolated dimer is considered first since its static magnetic susceptibility can be calculated exactly. A slightly different derivation to the one given here can be found in Ref. [Joh97].

First of all, the Hamiltonian of a single dimer is given by

$$
H=J_{\text {intra }} \mathbf{S}_{1} \cdot \mathbf{S}_{2}=\frac{J_{\text {intra }}}{2}\left(S_{1}^{+} S_{2}^{-}+S_{1}^{-} S_{2}^{+}\right)+J_{\text {intra }} S_{1}^{z} S_{2}^{z}
$$

The Hamiltonian matrix is diagonal in the eigenbasis

$$
\begin{aligned}
& \left|E_{0}\right\rangle=\frac{1}{\sqrt{2}}(|\uparrow \downarrow\rangle-|\downarrow \uparrow\rangle), \quad\left|E_{1}\right\rangle=\frac{1}{\sqrt{2}}(|\uparrow \downarrow\rangle+|\downarrow \uparrow\rangle), \\
& \left|E_{2}\right\rangle=|\uparrow \uparrow\rangle, \quad\left|E_{3}\right\rangle=|\downarrow \downarrow\rangle
\end{aligned}
$$

with the corresponding eigenenergies

$$
E_{0}=-\frac{3}{4} J_{\text {intra }} \quad \text { and } \quad E_{1}=E_{2}=E_{3}=\frac{1}{4} J_{\text {intra }}
$$

The singlet ground state $\left|E_{0}\right\rangle$ of the dimer is separated by an energy gap of $J_{\text {intra }}$ from the degenerate triplet excitations $\left|E_{n}\right\rangle$ for $n=1,2,3$. The static magnetic susceptibility is defined as [Nol14]

$$
\chi=\lim _{h \rightarrow 0} \frac{\partial M(h)}{\partial h}=-\lim _{h \rightarrow 0} \frac{\partial^{2} F}{\partial^{2} h}=\lim _{h \rightarrow 0} \beta\left[\left\langle\left(S^{z}\right)^{2}\right\rangle_{h}-\left\langle S_{z}\right\rangle_{h}^{2}\right] g^{2} \mu_{\mathrm{B}}^{2}
$$

in the limit of zero magnetic field $h \rightarrow 0$. Here $F$ denotes the free energy, $Z$ the partition function:

$$
F=-\frac{1}{\beta} \ln Z, \quad Z=\operatorname{Tr} \mathrm{e}^{-\beta\left(H-g \mu_{\mathrm{B}} h S_{z}\right)} .
$$

The constant $\mu_{\mathrm{B}}$ is the Bohr magneton. The inverse temperature is given by $\beta=1 /\left(k_{\mathrm{B}} T\right)$ and $S^{z}=S_{1}^{z}+S_{2}^{z}$ represents the total spin operator of the dimer. Using the expression for a finite-temperature expectation value in the canonical ensemble in Eq. (2.10) and the eigenbasis in Eqs. (B.2a) and (B.2b), the expectation value $\left\langle\left(S^{z}\right)^{2}\right\rangle$ in Eq. (B.4) above can be stated as

$$
\left\langle\left(S^{z}\right)^{2}\right\rangle_{h=0}=\frac{1}{Z} \operatorname{Tr}\left[\left(S^{z}\right)^{2} \mathrm{e}^{-\beta H}\right]=\frac{1}{Z} \sum_{n=0}^{3} \mathrm{e}^{-\beta E_{n}}\left\langle E_{n}\left|\left(S^{z}\right)^{2}\right| E_{n}\right\rangle,
$$


where after the last equality one may write

$$
Z=\left(\sum_{n=0}^{3} \mathrm{e}^{-\beta E_{n}}\right)=\mathrm{e}^{\frac{3}{4} J_{\text {intra }} \beta}+3 \mathrm{e}^{-\frac{1}{4} J_{\text {intra }} \beta} .
$$

Due to

$$
\left\langle E_{0}\left|\left(S^{z}\right)^{2}\right| E_{0}\right\rangle=\left\langle E_{1}\left|\left(S^{z}\right)^{2}\right| E_{1}\right\rangle=0 \quad \text { and } \quad\left\langle E_{2}\left|\left(S^{z}\right)^{2}\right| E_{2}\right\rangle=\left\langle E_{3}\left|\left(S^{z}\right)^{2}\right| E_{3}\right\rangle=1
$$

this yields

$$
\left\langle\left(S^{z}\right)^{2}\right\rangle_{h=0}=\frac{2}{\mathrm{e}^{J_{\text {intra }} \beta}+3} .
$$

Similarly, one finds $\left\langle S^{z}\right\rangle_{h=0}=0$ and thus the static susceptibility of a single dimer is given by

$$
\chi_{\text {single dimer }}=g^{2} \mu_{\mathrm{B}}^{2} \beta \frac{2}{\mathrm{e}^{J_{\text {intra }} \beta}+3} .
$$

If one considers a completely dimerized system of $L$ magnetic atoms, there are $L / 2$ noninteracting dimers. Their static susceptibility is

$$
\chi_{\text {dimer }}(T)=\frac{g^{2} \mu_{\mathrm{B}}^{2}}{k_{\mathrm{B}} T} \frac{L}{2} \frac{2}{\mathrm{e}^{J_{\text {intra }} /\left(k_{\mathrm{B}} T\right)}+3}=\frac{3 C}{T} \frac{1}{\mathrm{e}^{J_{\text {intra }} /\left(k_{\mathrm{B}} T\right)}+3},
$$

where in the last step the Curie constant $C$ was introduced [Joh97]. By means of a molecular field approximation, weak interdimer couplings can be included as effective fields. Following Ref. [SJ07] the susceptibility of the weakly interacting dimers reads

$$
\chi_{\text {int. dimer }}(T)=\frac{\chi_{\text {dimer }}(T)}{1+\gamma \chi_{\text {dimer }}(T)}=\frac{3 C / T}{3+\mathrm{e}^{J_{\text {intra }} /\left(k_{\mathrm{B}} T\right)}+\gamma 3 C / T} .
$$

Inserting the molecular field constant $\gamma=J_{\text {inter }} /(3 C)$ yields

$$
\chi_{\text {int. dimer }}(T)=\frac{3 C / T}{3+\mathrm{e}^{J_{\text {intra }} /\left(k_{\mathrm{B}} T\right)}+J_{\text {inter }} / T} .
$$

This result is used for the fitting analysis of the experimental static magnetic susceptibility data in Sec. 7.3.1.

\section{\begin{tabular}{l|l} 
Intradimer correlation function & B.2
\end{tabular}}

Partly based on the results of the previous section, the exact evaluation of the intradimer correlation function $\left\langle\mathbf{S}_{1} \cdot \mathbf{S}_{2}\right\rangle$ of an isolated dimer as a function of temperature is briefly sketched here. As $\mathbf{S}_{1} \cdot \mathbf{S}_{2}$ is closely related to the Hamiltonian in Eq. (B.1), one finds the matrix elements

$$
\left\langle E_{0}\left|\mathbf{S}_{1} \cdot \mathbf{S}_{2}\right| E_{0}\right\rangle=-\frac{3}{4} \quad \text { and } \quad\left\langle E_{i}\left|\mathbf{S}_{1} \cdot \mathbf{S}_{2}\right| E_{n}\right\rangle=\frac{1}{4} \quad \text { for } \quad n=1,2,3 .
$$

With these intermediate results, the correlation function can be calculated as

$$
\begin{array}{r}
\left\langle\mathbf{S}_{1} \cdot \mathbf{S}_{2}\right\rangle_{\text {isolated }}(T)=\sum_{n=0}^{3} \mathrm{e}^{-\beta E_{n}}\left\langle E_{n}\left|\mathbf{S}_{1} \cdot \mathbf{S}_{2}\right| E_{n}\right\rangle /\left(\sum_{n=0}^{3} \mathrm{e}^{-\beta E_{n}}\right) \\
=\frac{3}{4} \frac{1-\mathrm{e}^{J_{\text {intra }} /\left(k_{\mathrm{B}} T\right)}}{3+\mathrm{e}^{J_{\text {intra }} /\left(k_{\mathrm{B}} T\right)}}=-\frac{3}{4} \frac{1-\mathrm{e}^{-J_{\text {intra }} /\left(k_{\mathrm{B}} T\right)}}{1+3 e^{-J_{\text {intra }} /\left(k_{\mathrm{B}} T\right)}} .
\end{array}
$$

The result in Eq. (B.16) is used in Sec. 7.4.3. 


\section{Temperature dependence of $J_{1}$ in $\mathrm{BaCu}_{2} \mathrm{~V}_{2} \mathrm{O}_{8}$}

The present section summarizes results from the supplemental material of Ref. [KTF $\left.{ }^{+} 16\right]$.

Since the INS measurements are performed over a broad temperature range, the presence of lattice distortion may for instance lead to slight changes of the values $J_{1}$ and $J_{2}$ for the exchange interactions. Such small temperature dependencies of the couplings are observed as a shift of peak positions at finite temperature. In general, an observed shift occurs as a combination of two factors. First, thermal effects intrinsic to the magnetic system play a role, which are included in the DMRG computations. Secondly, the aforementioned temperature dependence of $J_{1}$ and/or $J_{2}$ causes an energy shift of the excitations. The latter effect is not taken into account by the DMRG calculations since the intradimer coupling $J_{\text {intra }}$ is set to unity and $J_{\text {inter }}=J_{2} / J_{1}$ is assumed to be constant - irrespective of temperature.

In a first step, the temperature dependence of $J_{1}$ and $J_{2}$ is neglected. Consequently, the DMRG results are scaled with a fixed value $J_{1}=40.92 \mathrm{meV}$ at all temperatures and compared to the INS data in Fig. B.1(a). With increasing temperature the DMRG peak positions are consistently found at higher energy than the experimentally observed excitations. These offsets grow only moderately with temperature and are roughly of the same magnitude at both the minima and maxima. Therefore it is justified to assume that only $J_{1}$ is temperature dependent as its value shifts the center of the band (CB). By analyzing the CB for both DMRG and experimental results, the dependence $J_{1}(T)$ stated
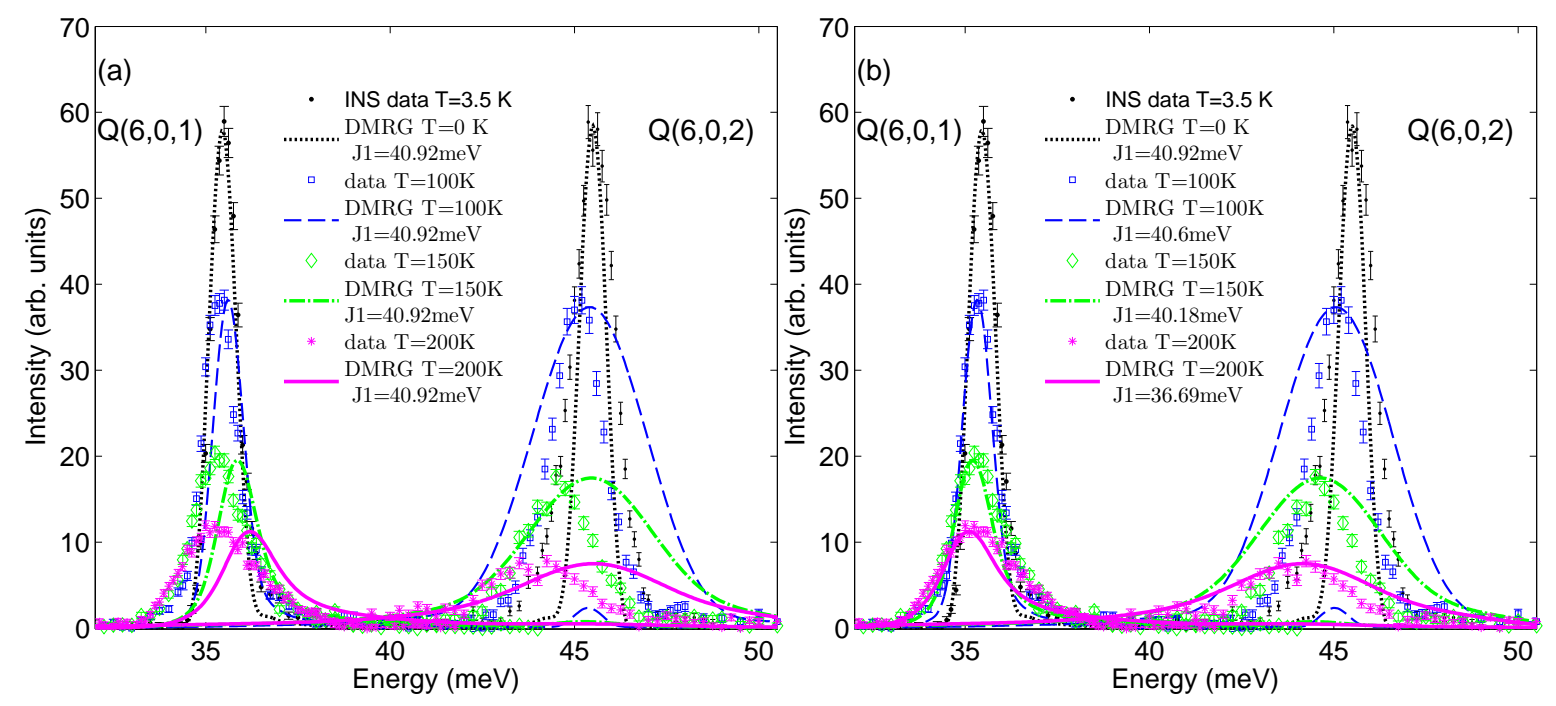

Figure B.1: (Taken from the supplemental material of Ref. $\left[\mathrm{KTF}^{+} 16\right]$.) Comparison of DMRG results obtained in this thesis and experimental data at the dispersion minimum $(6,0,1)$ and maximum $(6,0,2)$ at temperatures of $T=3.5 \mathrm{~K}, 100 \mathrm{~K}, 150 \mathrm{~K}$, and $200 \mathrm{~K}$ in order to assess the temperature dependence of the exchange coupling $J_{1}$. Note that the Gaussian resolution $\eta=0.035$ (corresponding to $W_{G} \approx 3.37 \mathrm{meV}$ ) of the DMRG data at $(6,0,2)$ is lower than the experimental one but sufficiently high to reliably determine the peak position. (a) The DMRG calculations are scaled by the fixed value of $J_{1}=40.92 \mathrm{meV}$ at all temperatures. (b) The DMRG computations are scaled by the temperature dependent values of $J_{1}=40.92 \mathrm{meV}, 40.60 \mathrm{meV}$, $40.18 \mathrm{meV}$, and $39.69 \mathrm{meV}$ at $T=0 \mathrm{~K}, 100 \mathrm{~K}, 150 \mathrm{~K}$, and $200 \mathrm{~K}$. 


\begin{tabular}{c|c|c|c}
\hline \hline $\begin{array}{c}\text { Temperature } \\
(\mathrm{K})\end{array}$ & $\begin{array}{c}\text { CB experiment } \\
(\mathrm{meV})\end{array}$ & $\begin{array}{c}\text { CB DMRG } \\
\left(E / J_{1}\right)\end{array}$ & $\begin{array}{c}J_{1}(T) \\
(\mathrm{meV})\end{array}$ \\
\hline 3.5 & $40.495 \pm 0.03$ & 0.9896 & $40.92 \pm 0.03$ \\
\hline 100 & $40.19 \pm 0.05$ & 0.9900 & $40.60 \pm 0.05$ \\
\hline 150 & $39.92 \pm 0.05$ & 0.9935 & $40.18 \pm 0.05$ \\
\hline 200 & $39.65 \pm 0.1$ & 0.9990 & $39.69 \pm 0.10$ \\
\hline \hline
\end{tabular}

Table B.1: Temperature dependence of the center of the band (CB) determined both from the experiment and DMRG in order to determine $J_{1}(T)$.

in Tab. B.1 is obtained. Thus, the DMRG data is scaled by these values in Fig. B.1(b) which shows excellent agreement for the peak positions. The corrections included in $J_{1}(T)$ are smaller than $5 \%$ compared to the base-temperature value of $J_{1}$.

\section{\begin{tabular}{l|l} 
Linear prediction & B.4
\end{tabular}}

As explained in Sec. 7.4.2, linear prediction has been used to enhance the resolution of the MPS-based Chebyshev expansions at finite temperature in Fig. 7.5 in order to compare them to the high-resolution data obtained in inelastic neutron scattering experiments. The scope of this appendix is to assess the accuracy of the linear prediction.

In a first step, the accuracy of the input for the linear prediction is studied. The first 1000 Chebyshev moments for the dynamical spin structure factor $S_{z z}(Q, \omega)$ at $Q=(6,0,1)$ are calculated for different numbers of the maximal internal bond dimension of the MPS: $m=150$ and $m=250$. A comparison of these data at different temperatures is presented in the top row of Fig. B.2. The bottom row of this figure shows that the absolute deviation between the results obtained for $m=150$ and $m=250$ which grows with the number of iterations and is still sufficiently small compared to the value of the Chebyshev moments. Therefore the first 1000 moments can be calculated accurately for $m=250$ and are used as an input for the linear prediction.

Now linear prediction (see Sec. 3.5.4) is performed in order to extrapolate the Chebyshev moments $\mu_{n}$ to $n>1000$. To this end, the $m=250$ data from Fig. B. 2 and the training interval $I_{\text {fit }}=[500,1000]$ are used. Figure B.3 shows the extrapolated moments in comparison to Chebyshev moments calculated without linear prediction using $m=150$. In Fig. B.3(b), also the Chebyshev moments calculated for $m=250$ are shown at $T=150 \mathrm{~K}$. The predicted moments display small but visible deviations from the results obtained without linear prediction. The discrepancies are most pronounced at the local extrema around $n \sim 1800$ and are about $20 \%$ for $m=150$ and roughly $10 \%$ for $m=250$. However, linear prediction extrapolates the moments using very accurate input data. Hence, one may assume that the prediction is also of high accuracy. Therefore it is not surprising that the continued calculation for a fixed value of $m=250$ collects further errors mainly as a consequence of the iterative compression of the Chebyshev vectors. Increasing deviations between the predicted moments and the calculated $m=150$ data with the number of iterations, as also seen in Figs. B.3(a) and B.3(c), can thus be expected. However, the crucial question is how large the effect of potential deviations in the predicted Chebyshev moments will be for the spectral lineshape. This is considered in the following. 


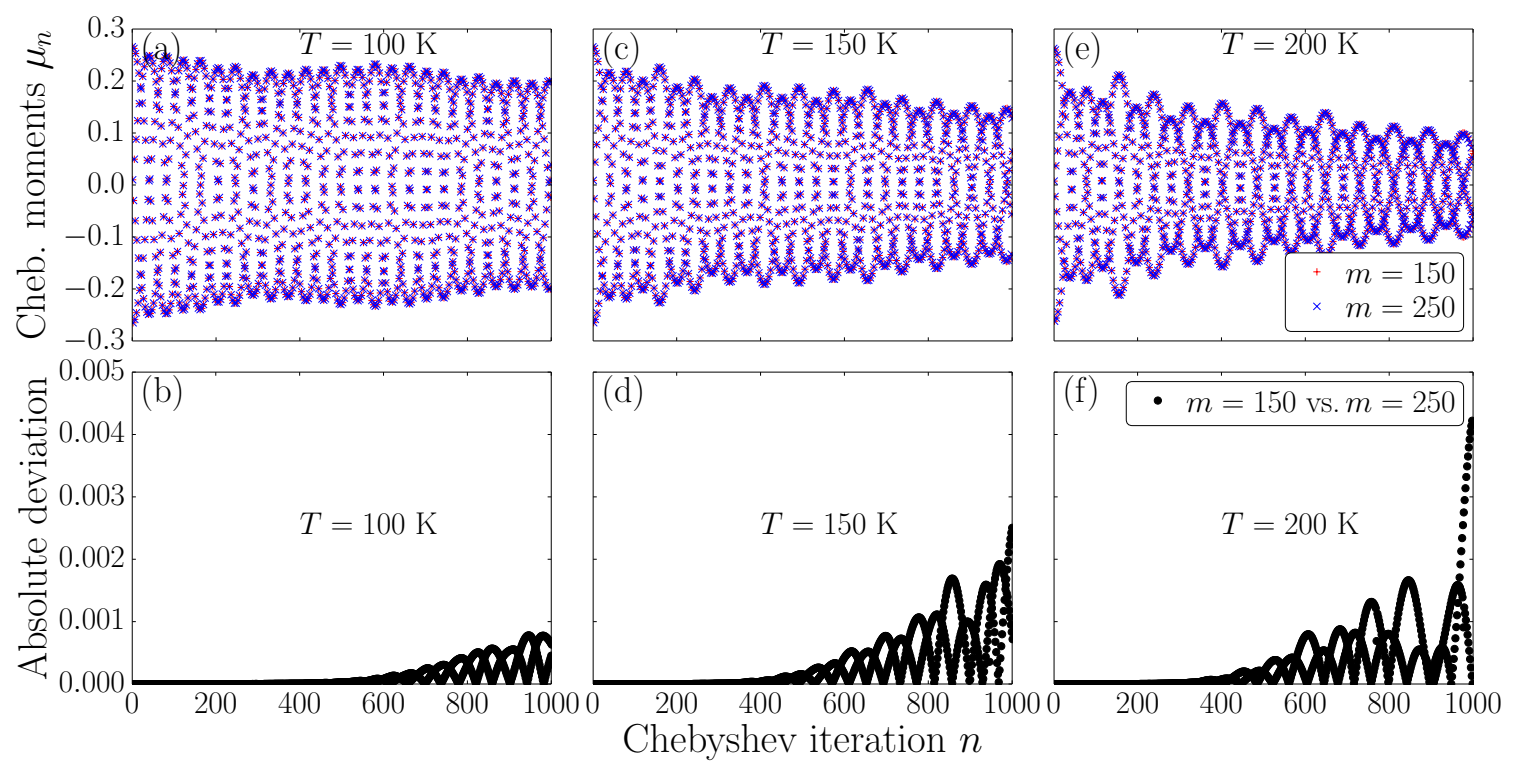

Figure B.2: (Top row) Chebyshev moments for the dynamical spin structure factor $S_{z z}(Q, \omega)$ at $Q=(6,0,1)$ for $J_{\text {inter }} / J_{\text {intra }}=-0.29$ in dependence of the iteration. In order to check the accuracy, the results are calculated both with a maximal internal MPS bond dimension of $m=150$ and $m=250$ at different temperatures: (a) $T=100 \mathrm{~K}$, (c) $150 \mathrm{~K}$, and (e) $200 \mathrm{~K}$. (Bottom row) The absolute deviation between the computed Chebyshev moments calculated for an internal MPS bond dimension of $m=150$ and $m=250$ is depicted versus the iteration number for the same temperatures as in the top row: (b) $T=100 \mathrm{~K}$, (d) $150 \mathrm{~K}$, and (f) $200 \mathrm{~K}$. The small deviations in the Chebyshev moments confirm a very high accuracy of the MPS calculations.

Due to the slow decay of the Chebyshev moments for the dynamical spin structure factor $S_{z z}(Q, \omega)$ at $Q=(6,0,1)$, it is not reasonable to discard the damping factors $g_{n}$ in the Chebyshev expansion as proposed in recent developments concerning the calculation of spectral functions at $T=0$, see Sec. 3.5.4. Instead, the $g_{n}$ from Eq. (3.79) introducing a Gaussian broadening of the spectral function are kept. The experimental resolution is reproduced for an expansion order of $N \approx 8390$. In order to get an estimate of the errors obtained in the lineshape, the spectral function is obtained by linear prediction using the two different fitting intervals $I_{\text {fit }}=[500,1000]$ in combination with $m=250$ and $I_{\text {fit }}=[1000,2000]$ (mostly $m=150$ ). The corresponding results for the dynamical spin structure factor $S_{z z}(Q, \omega)$ at $Q=(6,0,1)$ are shown in Fig. B.4. It turns out that there is only a slight dependence of the lineshape on $I_{\text {fit }}$ and $m$ since the deviations are small. Therefore the data using $I_{\text {fit }}=[500,1000]$ are shown in Fig. 7.5. The discrepancies are most pronounced at $T=200 \mathrm{~K}$ in Fig. B.4(c).

In order to obtain the Lorentzian width $W_{L}$ and asymmetry parameter $\alpha$ needed for a quantitative comparison to the experiment, the fitting function in Eq. (7.10) is not only fitted to a single data set at each temperature but rather to the spectral functions obtained for both $I_{\text {fit }}=[500,1000]$ and $I_{\text {fit }}=[1000,2000]$ in Fig. B.4. In addition, there is also a slight dependence on the frequency interval chosen for the fit. This effect is also considered to obtain the error estimates for the Lorentzian width $W_{L}$ and asymmetry parameter $\alpha$ depicted in Figs. 7.5(g)-(h). These error estimates reflect the maximal deviation found for the two training intervals $I_{\text {fit }}$ and different frequency windows for fitting the function in Eq. (7.10) to the MPS data. 

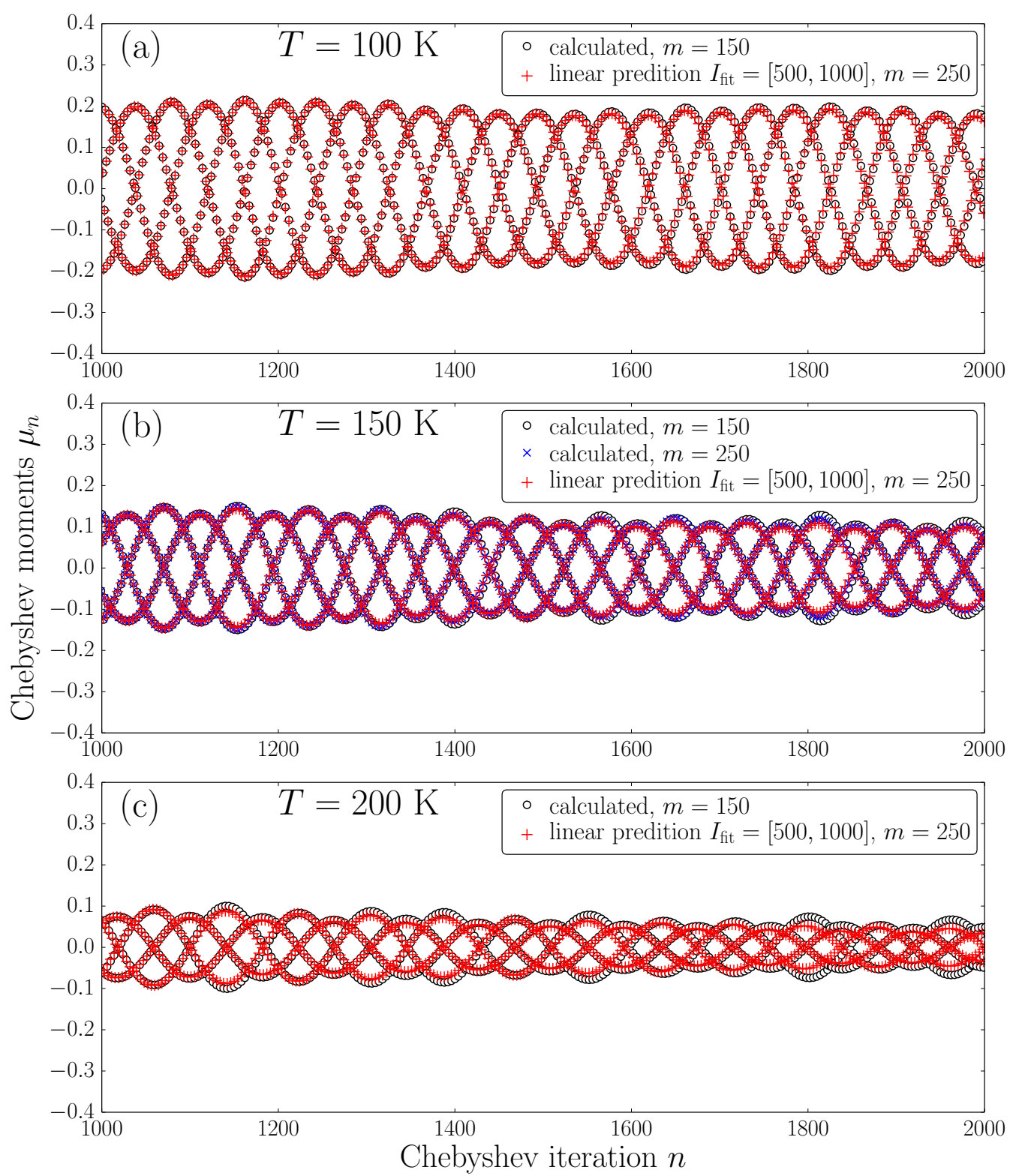

Figure B.3: Comparison between the calculated Chebyshev moments for the dynamical spin structure factor $S_{z z}(Q, \omega)$ at $Q=(6,0,1)\left(J_{\text {inter }} / J_{\text {intra }}=-0.29\right)$ and the Chebyshev moments extrapolated by linear prediction using the fitting interval $I_{\text {fit }}=[500,1000]$. Data computed with a maximal internal MPS bond dimension of $m=250$ is used for the linear prediction. The results are presented at different temperatures: (a) $T=100 \mathrm{~K}$, (b) $150 \mathrm{~K}$, and (c) $200 \mathrm{~K}$. The Chebyshev moments calculated without linear prediction are shown for $m=150$ and also for $m=250$ in panel (b). Note that the horizontal axis starts at $n=1000$ in all panels since the data is not predicted for smaller iteration numbers. 

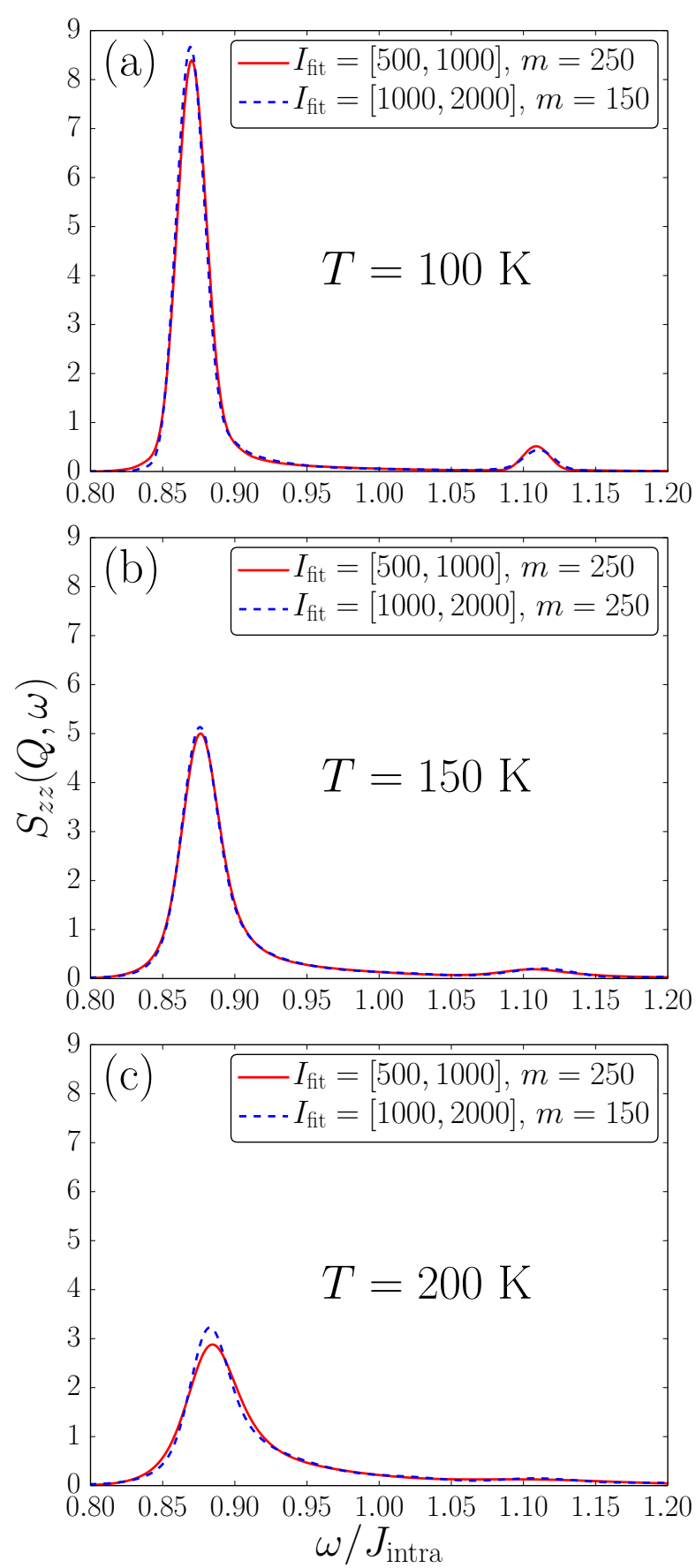

Figure B.4: Dynamical spin structure factor $S_{z z}(Q, \omega)$ at $Q=(6,0,1)$ obtained by linear prediction for the Chebyshev moments at (a) $T=100 \mathrm{~K}$, (b) $150 \mathrm{~K}$, and (c) $200 \mathrm{~K}$ using $J_{\text {inter }} / J_{\text {intra }}=-0.29$. The Chebyshev-expansions are shown at a Gaussian broadening of $\eta=$ $W_{G} /(2 \ln 2)=0.0086$ corresponding to an expansion order of $N \approx 8390$. In each panel results for two different fitting intervals $I_{\text {fit }}$ are shown in order to assess their effect on the spectral lineshape. 

List of references 



\section{In alphabetical order of labels}

[ACL12] R. Augusiak, M. F. Cucchietti, and M. Lewenstein. Modern Theories of Many-Particle Systems in Condensed Matter Physics, chapter Many-Body Physics from a Quantum Information Perspective, pages 245-294. Springer, Berlin, Heidelberg (2012).

URL http://dx.doi.org/10.1007/978-3-642-10449-7_6

[ADEvdL03] M. Aichhorn, M. Daghofer, H. G. Evertz, and W. von der Linden. Low-temperature Lanczos method for strongly correlated systems. Physical Review B 67, 161103(R) (2003). URL http://dx.doi.org/10.1103/PhysRevB.67.161103

[Aff89] I. Affleck. Quantum spin chains and the Haldane gap. Journal of Physics: Condensed Matter 1(19), 3047 (1989).

URL http://dx.doi.org/10.1088/0953-8984/1/19/001

[AFOV08] L. Amico, R. Fazio, A. Osterloh, and V. Vedral. Entanglement in many-body systems. Reviews of Modern Physics 80, 517 (2008).

URL http://dx.doi.org/10.1103/RevModPhys.80.517

[Alv13] G. Alvarez. Production of minimally entangled typical thermal states with the Krylov-space approach. Physical Review B 87, 245130 (2013).

URL http://dx.doi.org/10.1103/PhysRevB.87.245130

[And50] P. W. Anderson. Antiferromagnetism. Theory of Superexchange Interaction. Physical Review 79, 350 (1950).

URL http://dx.doi.org/10.1103/PhysRev.79.350

[And63] P. W. Anderson. Theory of Magnetic Exchange Interactions: Exchange in Insulators and Semiconductors. volume 14 of Solid State Physics, pages 99 - 214. Academic Press (1963). URL http://dx.doi.org/10.1016/S0081-1947(08)60260-X

$\left[\mathrm{ANI}^{+} 00\right] \quad$ T. Asano, H. Nojiri, Y. Inagaki, J. P. Boucher, T. Sakon, Y. Ajiro, and M. Motokawa. ESR Investigation on the Breather Mode and the Spinon-Breather Dynamical Crossover in Cu Benzoate. Physical Review Letters 84, 5880 (2000).

URL http://dx.doi.org/10.1103/PhysRevLett.84.5880

$\left[\mathrm{ANI}^{+} 03\right]$ T. Asano, H. Nojiri, Y. Inagaki, T. Sakon, J.-P. Boucher, Y. Ajiro, and M. Motokawa. ESR study of sine-Gordon excitations in $S=1 / 2$ antiferromagnetic chain: copper benzoate. Physica B: Condensed Matter 329-333, 1213 (2003).

URL http://dx.doi.org/10.1016/S0921-4526(02)02137-3

[AO99] I. Affleck and M. Oshikawa. Field-induced gap in Cu benzoate and other $S=\frac{1}{2}$ antiferromagnetic chains. Physical Review B 60, 1038 (1999).

URL http://dx.doi.org/10.1103/PhysRevB.60.1038

[AU85] T. Arimitsu and H. Umezawa. A General Formulation of Nonequilibrium Thermo Field Dynamics. Progress of Theoretical Physics 74(2), 429 (1985).

URL http://dx.doi.org/10.1143/PTP.74.429

[AU87] T. Arimitsu and H. Umezawa. Non-Equilibrium Thermo Field Dynamics. Progress of Theoretical Physics 77(1), 32 (1987).

URL http://dx.doi.org/10.1143/PTP.77.32

[Aue94] A. Auerbach. Interacting Electrons and Quantum Magnetism. Springer, New York (1994). URL http://dx.doi.org/10.1007/978-1-4612-0869-3

[Bar13] T. Barthel. Precise evaluation of thermal response functions by optimized density matrix renormalization group schemes. New Journal of Physics 15(7), 073010 (2013).

URL http://dx.doi.org/10.1088/1367-2630/15/7/073010 
[BB15] M. Binder and T. Barthel. Minimally entangled typical thermal states versus matrix product purifications for the simulation of equilibrium states and time evolution. Physical Review B 92, 125119 (2015).

URL http://dx.doi.org/10.1103/PhysRevB.92.125119

[BD87] S. M. Barnett and B. J. Dalton. Liouville space description of thermofields and their generalisations. Journal of Physics A: Mathematical and General 20(2), 411 (1987). URL http://dx.doi.org/10.1088/0305-4470/20/2/026

[BD05] D. Baeriswyl and L. Degiorgi, editors. Strong Interactions in Low Dimensions. Kluwer Academic Publishers, Dordrecht (2005).

[Bea04] K. S. D. Beach. Identifying the maximum entropy method as a special limit of stochastic analytic continuation. arXiv:cond-mat/0403055 [cond-mat.str-el] (2004).

[Bet31] H. Bethe. Zur Theorie der Metalle. Zeitschrift für Physik 71(3), 205 (1931). URL http://dx.doi.org/10.1007/BF01341708

[BF04] H. Bruus and K. Flensberg. Many-Body Quantum Theory in Condensed Matter Physics. Oxford University Press, Oxford, 1st edition (2004).

[BGJ04] H. Benthien, F. Gebhard, and E. Jeckelmann. Spectral Function of the One-Dimensional Hubbard Model away from Half Filling. Physical Review Letters 92, 256401 (2004). URL http://dx.doi.org/10.1103/PhysRevLett.92.256401

[BH54] M. Born and K. Huang. Dynamical Theory of Crystal Lattices. The Clarendon Press, Oxford (1954).

$\left[\mathrm{BHM}^{+} 12\right] \quad$ L. Bonnes, K. R. A. Hazzard, S. R. Manmana, A. M. Rey, and S. Wessel. Adiabatic Loading of One-Dimensional SU(N) Alkaline-Earth-Atom Fermions in Optical Lattices. Physical Review Letters 109, 205305 (2012).

URL http://dx.doi.org/10.1103/PhysRevLett.109.205305

[BHV06] S. Bravyi, M. B. Hastings, and F. Verstraete. Lieb-Robinson Bounds and the Generation of Correlations and Topological Quantum Order. Physical Review Letters 97, 050401 (2006). URL http://dx.doi.org/10.1103/PhysRevLett.97.050401

[BKHK06] S. P. Bayrakci, T. Keller, K. Habicht, and B. Keimer. Spin-Wave Lifetimes Throughout the Brillouin Zone. Science 312, 1926 (2006).

URL http://dx.doi.org/10.1126/science.1127756

[Blu01] S. Blundell. Magnetism in Condensed Matter. Oxford, Oxford (2001).

[Boy89] J. P. Boyd. Chebyshev and Fourier Spectral Methods. Springer, Berlin (1989).

[BRT99] T. Barnes, J. Riera, and D. A. Tennant. $S=1 / 2$ alternating chain using multiprecision methods. Physical Review B 59, 11384 (1999).

URL http://dx.doi.org/10.1103/PhysRevB.59.11384

[BS14] A. Braun and P. Schmitteckert. Numerical evaluation of Green's functions based on the Chebyshev expansion. Physical Review B 90, 165112 (2014).

URL http://dx.doi.org/10.1103/PhysRevB.90.165112

[BSW09] T. Barthel, U. Schollwöck, and S. R. White. Spectral functions in one-dimensional quantum systems at finite temperature using the density matrix renormalization group. Physical Review B 79, 245101 (2009).

URL http://dx.doi.org/10.1103/PhysRevB.79.245101

[BT79] H. Bergknoff and H. B. Thacker. Structure and solution of the massive Thirring model. Physical Review D 19, 3666 (1979).

URL http://dx.doi.org/10.1103/PhysRevD.19.3666

$\left[\mathrm{BTL}^{+} 13\right] \quad$ S. P. Bayrakci, D. A. Tennant, P. Leininger, T. Keller, M. C. R. Gibson, S. D. Wilson, R. J. Birgeneau, and B. Keimer. Lifetimes of Antiferromagnetic Magnons in Two and Three Dimensions: Experiment, Theory, and Numerics. Physical Review Letters 111, 017204 (2013).

URL http://dx.doi.org/10.1103/PhysRevLett.111.017204 
[BvDW15] B. Bruognolo, J. von Delft, and A. Weichselbaum. Symmetric minimally entangled typical thermal states. Physical Review B 92, 115105 (2015).

URL http://dx.doi.org/10.1103/PhysRevB.92.115105

[BWvDG16] B. Bruognolo, A. Weichselbaum, J. von Delft, and M. Garst. Dynamic structure factor of the spin-1/2 XXZ chain in a transverse field. arXiv:1606.03294 [cond-mat.str-el] (2016).

[BXG96] R. J. Bursill, T. Xiang, and G. A. Gehring. The density matrix renormalization group for a quantum spin chain at non-zero temperature. Journal of Physics: Condensed Matter 8(40), L583 (1996).

URL http://dx.doi.org/10.1088/0953-8984/8/40/003

[Bü03] A. Bühler. High temperature series expansions for spin and spin phonon systems. Ph.D. thesis, Universität Köln (2003).

URL http://d-nb.info/969818203

[CC04] P. Calabrese and J. Cardy. Entanglement entropy and quantum field theory. Journal of Statistical Mechanics: Theory and Experiment 2004(6), P06002 (2004).

URL http://dx.doi.org/10.1088/1742-5468/2004/06/P06002

[CH06] J.-S. Caux and R. Hagemans. The four-spinon dynamical structure factor of the Heisenberg chain. Journal of Statistical Mechanics: Theory and Experiment 2006(12), P12013 (2006). URL http://dx.doi.org/10.1088/1742-5468/2006/12/P12013

[CL00] P. M. Chaikin and T. C. Lubensky. Principles of condensed matter physics. Cambridge University Press, Cambridge (2000).

[Cor16] P. Corboz. Improved energy extrapolation with infinite projected entangled-pair states applied to the two-dimensional Hubbard model. Physical Review B 93, 045116 (2016). URL http://dx.doi.org/10.1103/PhysRevB.93.045116

[CW02] J. K. Cullum and R. A. Willoughby. Lanczos Algorithms for Large Symmetric Eigenvalue Computations. Society for Industrial Mathematics (2002).

[Dag94] E. Dagotto. Correlated electrons in high-temperature superconductors. Reviews of Modern Physics 66, 763 (1994).

URL http://dx.doi.org/10.1103/RevModPhys.66.763

[Dal82] B. J. Dalton. Liouville space theory of sequential quantum processes. I. General theory. Journal of Physics A: Mathematical and General 15(7), 2157 (1982).

URL http://dx.doi.org/10.1088/0305-4470/15/7/026

[Dar12] P. E. Dargel. Spectral functions of low-dimensional quantum systems. Ph.D. thesis, GeorgAugust-Universität Göttingen (2012).

URL http://hdl .handle.net/11858/00-1735-0000-000D-F1A3-6

[dCP62] J. des Cloizeaux and J. J. Pearson. Spin-Wave Spectrum of the Antiferromagnetic Linear Chain. Physical Review 128, 2131 (1962).

URL http://dx.doi.org/10.1103/PhysRev.128.2131

[DDacR ${ }^{+}$96] D. C. Dender, D. Davidović, D. H. Reich, C. Broholm, K. Lefmann, and G. Aeppli. Magnetic properties of a quasi-one-dimensional $S=1 / 2$ antiferromagnet: Copper benzoate. Physical Review B 53, 2583 (1996).

URL http://dx.doi.org/10.1103/PhysRevB.53.2583

$\left[\mathrm{DHP}^{+} 11\right] \quad$ P. E. Dargel, A. Honecker, R. Peters, R. M. Noack, and T. Pruschke. Adaptive Lanczosvector method for dynamic properties within the density matrix renormalization group. Physical Review B 83, 161104(R) (2011).

URL http://dx.doi.org/10.1103/PhysRevB.83.161104

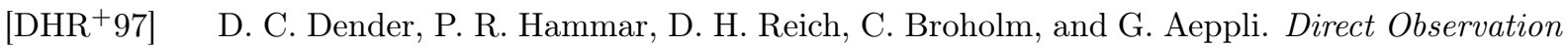
of Field-Induced Incommensurate Fluctuations in a One-Dimensional $S=1 / 2$ Antiferromagnet. Physical Review Letters 79, 1750 (1997).

URL http://dx.doi.org/10.1103/PhysRevLett.79.1750 
[DK98] O. Derzhko and T. Krokhmalskii. Numerical Approach for the Study of the Spin-1/2 XY Chains Dynamic Properties. Physica Status Solidi B 208(1), 221 (1998).

URL http://dx.doi.org/10.1002/(SICI) 1521-3951(199807) 208:1<221: : AID-PSSB221>3.0.CO;2-E

[DKS00] O. Derzhko, T. Krokhmalskii, and J. Stolze. Dynamics of the spin-1/2 isotropic XY chain in a transverse field. Journal of Physics A: Mathematical and General 33(16), 3063 (2000). URL http://dx.doi.org/10.1088/0305-4470/33/16/301

[DKSV04] A. J. Daley, C. Kollath, U. Schollwöck, and G. Vidal. Time-dependent density-matrix renormalization-group using adaptive effective Hilbert spaces. Journal of Statistical Mechanics: Theory and Experiment 2004(04), P04005 (2004).

URL http://dx.doi.org/10.1088/1742-5468/2004/04/P04005

[DS98] K. Damle and S. Sachdev. Spin dynamics and transport in gapped one-dimensional Heisenberg antiferromagnets at nonzero temperatures. Physical Review B 57, 8307 (1998).

URL http://dx.doi.org/10.1103/PhysRevB.57.8307

[DS05] K. Damle and S. Sachdev. Universal Relaxational Dynamics of Gapped One-Dimensional Models in the Quantum Sine-Gordon Universality Class. Physical Review Letters 95, $187201(2005)$.

URL http://dx.doi.org/10.1103/PhysRevLett.95.187201

[DV06] O. Derzhko and T. Verkholyak. Dynamic Structure Factors of the Spin-1/2 XX Chain with Dzyaloshinskii-Moriya Interaction. Journal of the Physical Society of Japan 75(10), 104711 (2006).

URL http://dx.doi.org/10.1143/JPSJ.75.104711

[DVKB06] O. Derzhko, T. Verkholyak, T. Krokhmalskii, and H. Büttner. Dynamic probes of quantum spin chains with the Dzyaloshinskii-Moriya interaction. Physical Review B 73, 214407 (2006).

URL http://dx.doi.org/10.1103/PhysRevB.73.214407

$\left[\mathrm{DWH}^{+} 12\right] \quad$ P. E. Dargel, A. Wöllert, A. Honecker, I. P. McCulloch, U. Schollwöck, and T. Pruschke. Lanczos algorithm with matrix product states for dynamical correlation functions. Physical Review B 85, 205119 (2012).

URL http://dx.doi.org/10.1103/PhysRevB.85.205119

[DWK07] K. Doll, A. U. B. Wolter, and H.-H. Klauss. Electronic structure of the molecule-based magnet $\mathrm{CuPM}\left(\mathrm{NO}_{3}\right)_{2}\left(\mathrm{H}_{2} \mathrm{O}\right)_{2}$. Physical Review B 75, 184433 (2007).

URL http://dx.doi.org/10.1103/PhysRevB.75.184433

[DYMT70] M. Date, H. Yamazaki, M. Motokawa, and S. Tazawa. Linear Chain Antiferromagnetism in Copper Benzoate. Progress of Theoretical Physics Supplement 46, 194 (1970).

URL http://dx.doi.org/10.1143/PTPS.46.194

[Dzy58] I. Dzyaloshinsky. A thermodynamic theory of "weak" ferromagnetism of antiferromagnetics. Journal of Physics and Chemistry of Solids 4(4), 241 (1958).

URL http://dx.doi.org/10.1016/0022-3697(58)90076-3

[EAT94] S. Eggert, I. Affleck, and M. Takahashi. Susceptibility of the spin 1/2 Heisenberg antiferromagnetic chain. Phys. Rev. Lett. 73, 332 (1994).

URL http://dx.doi.org/10.1103/PhysRevLett.73.332

[Eco06] E. N. Economou. Green's Functions in Quantum Physics, volume 7 of Springer Series in Solid-State Sciences. Springer, Berlin, Heidelberg, 3rd edition (2006).

URL http://dx.doi.org/10.1007/3-540-28841-4

[ECP10] J. Eisert, M. Cramer, and M. B. Plenio. Colloquium: Area laws for the entanglement entropy. Reviews of Modern Physics 82, 277 (2010).

URL http://dx.doi.org/10.1103/RevModPhys.82.277

$\left[\mathrm{EFG}^{+} 05\right] \quad$ F. H. L. Essler, H. Frahm, F. Göhmann, A. Klümper, and V. E. Korepin. The OneDimensional Hubbard Model. Cambridge University Press (2005).

URL http://dx.doi.org/10.1017/CB09780511534843 
[EFH03] F. H. L. Essler, A. Furusaki, and T. Hikihara. Dynamical structure factor in copper benzoate and other spin-1/2 antiferromagnetic chains. Physical Review B 68, 064410 (2003).

URL http://dx.doi.org/10.1103/PhysRevB.68.064410

[EK08] F. H. L. Essler and R. M. Konik. Finite-temperature lineshapes in gapped quantum spin chains. Physical Review B 78, 100403(R) (2008).

URL http://dx.doi.org/10.1103/PhysRevB.78.100403

[EK09] F. H. L. Essler and R. M. Konik. Finite-temperature dynamical correlations in massive integrable quantum field theories. Journal of Statistical Mechanics: Theory and Experiment 2009(09), P09018 (2009).

URL http://dx.doi.org/10.1088/1742-5468/2009/09/P09018

[EO06] J. Eisert and T. J. Osborne. General Entanglement Scaling Laws from Time Evolution. Physical Review Letters 97, 150404 (2006).

URL http://dx.doi.org/10.1103/PhysRevLett.97.150404

[Ess99] F. H. L. Essler. Sine-Gordon low-energy effective theory for copper benzoate. Physical Review B 59, 14376 (1999).

URL http://dx.doi.org/10.1103/PhysRevB.59.14376

[ET98] F. H. L. Essler and A. M. Tsvelik. Dynamical magnetic susceptibilities in copper benzoate. Physical Review B 57, 10592 (1998).

URL http://dx.doi.org/10.1103/PhysRevB.57.10592

[EV11] G. Evenbly and G. Vidal. Tensor Network States and Geometry. Journal of Statistical Physics 145(4), 891 (2011).

URL http://dx.doi.org/10.1007/s10955-011-0237-4

$\left[\mathrm{FAG}^{+} 00\right] \quad$ R. Feyerherm, S. Abens, D. Günther, T. Ishida, M. Meißner, M. Meschke, T. Nogami, and M. Steiner. Magnetic-field induced gap and staggered susceptibility in the $S=1 / 2$ chain $\left[\mathrm{PMCu}\left(\mathrm{NO}_{3}\right)_{2} \cdot\left(\mathrm{H}_{2} \mathrm{O}\right)_{2}\right]_{n}(\mathrm{PM}=$ pyrimidine $)$. Journal of Physics: Condensed Matter 12(39), 8495 (2000).

URL http://dx.doi.org/10.1088/0953-8984/12/39/312

[Fan57] U. Fano. Description of States in Quantum Mechanics by Density Matrix and Operator Techniques. Reviews of Modern Physics 29, 74 (1957).

URL http://dx.doi.org/10.1103/RevModPhys.29.74

[Faz99] P. Fazekas. Lecture Notes on Electron Correlation and Magnetism, volume 5 of Series in Modern Condensed Matter Physics. World Scientific, Singapore, New Jersey, London, Hong Kong (1999).

[FF10] A. E. Feiguin and G. A. Fiete. Spectral properties of a spin-incoherent Luttinger liquid. Physical Review B 81, 075108 (2010).

URL http://dx.doi.org/10.1103/PhysRevB.81.075108

[FO12] S. C. Furuya and M. Oshikawa. Boundary Resonances in $S=1 / 2$ Antiferromagnetic Chains Under a Staggered Field. Physical Review Letters 109, 247603 (2012).

URL http://dx.doi.org/10.1103/PhysRevLett.109.247603

[Fra13] E. Fradkin. Field Theories of Condensed Matter Physics. Cambridge University Press, Cambridge, UK, 2nd edition (2013).

[FSU14] B. Fauseweh, J. Stolze, and G. S. Uhrig. Finite-temperature line shapes of hard-core bosons in quantum magnets: A diagrammatic approach tested in one dimension. Physical Review B 90, 024428 (2014).

URL http://dx.doi.org/10.1103/PhysRevB.90.024428

[FSW08] H. Fehske, R. Schneider, and A. Weiße, editors. Computational Many-Particle Physics, volume 739 of Lecture Notes in Physics. Springer, Berlin, Heidelberg (2008).

URL http://dx.doi.org/10.1007/978-3-540-74686-7

[FT81] L. D. Faddeev and L. A. Takhtajan. What is the spin of a spin wave? Physics Letters A 85(6), 375 (1981).

URL http://dx.doi.org/10.1016/0375-9601(81) 90335-2 
[FU15] B. Fauseweh and G. S. Uhrig. Low-temperature thermodynamics of multiflavored hardcore bosons by the Brückner approach. Physical Review B 92, 214417 (2015).

URL http://dx.doi.org/10.1103/PhysRevB.92.214417

[Fu195] P. Fulde. Electron Correlations in Molecules and Solids, volume 100 of Springer Series in Solid-State Sciences. Springer, Berlin, Heidelberg (1995). URL http://dx.doi.org/10.1007/978-3-642-57809-0

[FW71] A. L. Fetter and J. D. Walecka. Quantum theory of many-particle systems. McGraw-Hill, San Francisco (1971).

[FW05] A. E. Feiguin and S. R. White. Finite-temperature density matrix renormalization using an enlarged Hilbert space. Physical Review B 72, 220401(R) (2005).

URL http://dx.doi.org/10.1103/PhysRevB.72.220401

[GB87] E. R. Gagliano and C. A. Balseiro. Dynamical Properties of Quantum Many-Body Systems at Zero Temperature. Physical Review Letters 59, 2999 (1987).

URL http://dx.doi.org/10.1103/PhysRevLett.59.2999

[GB09] S. Grossjohann and W. Brenig. Spin dynamics of the antiferromagnetic spin $-\frac{1}{2}$ chain at finite magnetic fields and intermediate temperatures. Physical Review B 79, 094409 (2009). URL http://dx.doi.org/10.1103/PhysRevB.79.094409

[Gia04] T. Giamarchi. Quantum Physics in One Dimension. Oxford University Press, Oxford (2004).

[GK99] F. Göhmann and V. E. Korepin. The Hubbard chain: Lieb-Wu equations and norm of the eigenfunctions. Physics Letters A 263, 293 (1999). URL http://dx.doi.org/10.1016/S0375-9601(99)00774-4

[GKE10] W. D. Goetze, U. Karahasanovic, and F. H. L. Essler. Low-temperature dynamical structure factor of the two-leg spin- $\frac{1}{2}$ Heisenberg ladder. Physical Review B 82, 104417 (2010). URL http://dx.doi.org/10.1103/PhysRevB.82.104417

[GL96] G. H. Golub and C. F. V. Loan. Matrix Computations. Johns Hopkins University Press, 3rd edition (1996).

$\left[\mathrm{GML}^{+} 11\right] \quad$ E. Gull, A. J. Millis, A. I. Lichtenstein, A. N. Rubtsov, M. Troyer, and P. Werner. Continuous-time Monte Carlo methods for quantum impurity models. Reviews of Modern Physics 83, 349 (2011).

URL http://dx.doi.org/10.1103/RevModPhys.83.349

[Goo55] J. B. Goodenough. Theory of the Role of Covalence in the Perovskite-Type Manganites [La, $M(\mathrm{II})] \mathrm{MnO}_{3}$. Physical Review 100, 564 (1955).

URL http://dx.doi.org/10.1103/PhysRev.100.564

[Goo58] J. B. Goodenough. An interpretation of the magnetic properties of the perovskite-type mixed crystals $\mathrm{La}_{1-x} \mathrm{Sr}_{x} \mathrm{CoO}_{3-\lambda}$. Journal of Physics and Chemistry of Solids 6, 287 (1958). URL http://dx.doi.org/10.1016/0022-3697(58)90107-0

[Goo60] J. B. Goodenough. Direct Cation-Cation Interactions in Several Oxides. Physical Review 117, $1442(1960)$.

URL http://dx.doi.org/10.1103/PhysRev.117.1442

[Goo08] J. B. Goodenough. Goodenough-Kanamori rule. Scholarpedia 3(10), 7382 (2008). URL http://dx.doi.org/10.4249/scholarpedia.7382

$\left[\mathrm{GPB}^{+} 05\right] \quad$ K. Ghoshray, B. Pahari, B. Bandyopadhyay, R. Sarkar, and A. Ghoshray. ${ }^{51} \mathrm{~V}$ NMR study of the quasi-one-dimensional alternating chain compound $\mathrm{BaCu}_{2} \mathrm{~V}_{2} \mathrm{O}_{8}$. Physical Review $\mathrm{B}$ 71, 214401 (2005).

URL http://dx.doi.org/10.1103/PhysRevB.71.214401

[GR06] J. J. García-Ripoll. Time evolution of Matrix Product States. New Journal of Physics 8(12), 305 (2006).

URL http://dx.doi.org/10.1088/1367-2630/8/12/305

[GTV $\left.{ }^{+} 14\right] \quad$ M. Ganahl, P. Thunström, F. Verstraete, K. Held, and H. G. Evertz. Chebyshev expansion for impurity models using matrix product states. Physical Review B 90, 045144 (2014).

URL http://dx.doi.org/10.1103/PhysRevB.90.045144 
[Gut63] M. C. Gutzwiller. Effect of Correlation on the Ferromagnetism of Transition Metals. Physical Review Letters 10, 159 (1963).

URL http://dx.doi.org/10.1103/PhysRevLett.10.159

[Hal95] K. A. Hallberg. Density-matrix algorithm for the calculation of dynamical properties of low-dimensional systems. Physical Review B 52, 9827(R) (1995).

URL http://dx.doi.org/10.1103/PhysRevB.52.R9827

[Har73] A. B. Harris. Alternating Linear Heisenberg Antiferromagnet: The Exciton Limit. Physical Review B 7, 3166 (1973).

URL http://dx.doi.org/10.1103/PhysRevB.7.3166

[Has07a] M. B. Hastings. An area law for one-dimensional quantum systems. Journal of Statistical Mechanics: Theory and Experiment 2007(08), P08024 (2007).

URL http://dx.doi.org/10.1088/1742-5468/2007/08/P08024

[Has07b] M. B. Hastings. Entropy and entanglement in quantum ground states. Physical Review B 76, 035114 (2007).

URL http://dx.doi.org/10.1103/PhysRevB.76.035114

[Hay80] R. Haydock. The recursive solution of the Schrödinger equation. Computer Physics Communications 20(1), 11 (1980).

URL http://dx.doi.org/10.1016/0010-4655(80)90101-0

$\left[\mathrm{HCO}^{+} 11\right] \quad$ J. Haegeman, J. I. Cirac, T. J. Osborne, I. Pižorn, H. Verschelde, and F. Verstraete. Time-Dependent Variational Principle for Quantum Lattices. Physical Review Letters 107, 070601 (2011).

URL http://dx.doi.org/10.1103/PhysRevLett.107.070601

[Hei28] W. Heisenberg. Zur Theorie des Ferromagnetismus. Zeitschrift für Physik 49(9), 619 (1928).

URL http://dx.doi.org/10.1007/BF01328601

[HHK72] R. Haydock, V. Heine, and M. J. Kelly. Electronic structure based on the local atomic environment for tight-binding bands. Journal of Physics C: Solid State Physics 5(20), 2845 (1972).

URL http://dx.doi.org/10.1088/0022-3719/5/20/004

[HK10] M. Z. Hasan and C. L. Kane. Colloquium: Topological insulators. Reviews of Modern Physics 82, 3045 (2010).

URL http://dx.doi.org/10.1103/RevModPhys.82.3045

[HKHH71] A. B. Harris, D. Kumar, B. I. Halperin, and P. C. Hohenberg. Dynamics of an Antiferromagnet at Low Temperatures: Spin-Wave Damping and Hydrodynamics. Physical Review B 3, 961 (1971).

URL http://dx.doi.org/10.1103/PhysRevB.3.961

[HKI04] Z. He, T. Kyômen, and M. Itoh. $\mathrm{BaCu}_{2} \mathrm{~V}_{2} \mathrm{O}_{8}$ : Quasi-one-dimensional alternating chain compound with a large spin gap. Physical Review B 69, 220407 (2004).

URL http://dx.doi.org/10.1143/JPSJS.71S.190

[HKM15] J. C. Halimeh, F. Kolley, and I. P. McCulloch. Chebyshev matrix product state approach for time evolution. Physical Review B 92, 115130 (2015).

URL http://dx.doi.org/10.1103/PhysRevB.92.115130

[HL97] M. Hochbruck and C. Lubich. On Krylov Subspace Approximations to the Matrix Exponential Operator. SIAM Journal on Numerical Analysis 34(5), 1911 (1997).

URL http://dx.doi.org/10.1137/S0036142995280572

[HL99] M. Hochbruck and C. Lubich. Exponential Integrators for Quantum-Classical Molecular Dynamics. BIT Numerical Mathematics 39(4), 620 (1999).

URL http://dx.doi.org/10.1023/A\%3A1022335122807

$\left[\mathrm{HLO}^{+}{ }^{14}\right]$ J. Haegeman, C. Lubich, I. Oseledets, B. Vandereycken, and F. Verstraete. Unifying time evolution and optimization with matrix product states. arXiv:1408.5056 [quant-ph] (2014). URL http://arxiv.org/abs/1408.5056 
[HMHB07] F. Heidrich-Meisner, A. Honecker, and W. Brenig. Transport in quasi one-dimensional spin-1/2 systems. The European Physical Journal Special Topics 151, 135- (2007). URL http://dx.doi.org/10.1140/epjst/e2007-00369-2

[HMSW15] C. Hubig, I. P. McCulloch, U. Schollwöck, and F. A. Wolf. Strictly single-site DMRG algorithm with subspace expansion. Physical Review B 91, 155115 (2015). URL http://dx.doi.org/10.1103/PhysRevB.91.155115

[Hol12] A. M. Holzner. DMRG studies of Chebyshev-expanded spectral functions and quantum impurity models. Ph.D. thesis, Ludwig-Maximilians-Universität München (2012). URL http://nbn-resolving.de/urn:nbn:de:bvb:19-139333

$\left[\mathrm{HTC}^{+} 08\right]$ T. Huberman, D. A. Tennant, R. A. Cowley, R. Coldea, and C. D. Frost. A study of the quantum classical crossover in the spin dynamics of the $2 D S=5 / 2$ antiferromagnet $\mathrm{Rb}_{2} \mathrm{MnF}_{4}$ : neutron scattering, computer simulations and analytic theories. Journal of Statistical Mechanics: Theory and Experiment 2008(05), P05017 (2008). URL http://dx.doi.org/10.1088/1742-5468/2008/05/P05017

[HTI06] Z. He, T. Taniyama, and M. Itoh. Magnetic behavior and structural feature of quasi-onedimensional $\mathrm{BaCu}_{2} \mathrm{~V}_{2} \mathrm{O}_{8}$ crystal. Journal of Magnetism and Magnetic Materials 306(2), 277 (2006).

URL http://dx.doi.org/10.1016/j.jmmm.2006.03.041

[Hub63] J. Hubbard. Electron Correlations in Narrow Energy Bands. Proceedings of the Royal Society London A 276, 238 (1963).

URL http://dx.doi.org/10.1098/rspa.1963.0204

[HW09] A. Honecker and S. Wessel. Magnetocaloric effect in quantum spin-s chains. Condensed Matter Physics 12(3), 399 (2009).

URL http://dx.doi.org/10.5488/CMP.12.3.399

[HWM+11] A. Holzner, A. Weichselbaum, I. P. McCulloch, U. Schollwöck, and J. von Delft. Chebyshev matrix product state approach for spectral functions. Physical Review B 83, 195115 (2011). URL http://dx.doi.org/10.1103/PhysRevB.83.195115

[IE03] T. Iitaka and T. Ebisuzaki. Algorithm for Linear Response Functions at Finite Temperatures: Application to ESR Spectrum of $S=1 / 2$ Antiferromagnet Cu Benzoate. Physical Review Letters 90, 047203 (2003).

URL http://dx.doi.org/10.1103/PhysRevLett.90.047203

[INN ${ }^{+}$97] T. Ishida, K. Nakayama, M. Nakagawa, W. Sato, Y. Ishikawa, M. Yasui, F. Iwasaki, and T. Nogami. Magnetic properties of pyrimidine-bridged copper(II) complexes. Synth. Metals 85(1-3), 1655 (1997).

URL http://dx.doi.org/10.1016/S0379-6779(97)80384-0

[Jac11] D. Jackson. Über die Genauigkeit der Annäherung stetiger Funktionen durch ganze rationale Funktionen gegebenen Grades und trigonometrische Summen gegebener Ordnung. Ph.D. thesis, Georg-August-Universität Göttingen (1911).

URL http://eudml .org/doc/204248

[Jac12] D. Jackson. On approximation by trigonometric sums and polynomials. Transanctions of the American Mathematical Society 13, 491 (1912). URL http://dx.doi.org/10.2307/1988583

[JcvPcv94] J. Jaklič and P. Prelovšek. Lanczos method for the calculation of finite-temperature quantities in correlated systems. Physical Review B 49, 5065(R) (1994).

URL http://dx.doi.org/10.1103/PhysRevB.49.5065

[Jec02] E. Jeckelmann. Dynamical density-matrix renormalization-group method. Physical Review B 66, 045114 (2002).

URL http://dx.doi.org/10.1103/PhysRevB.66.045114

[Jec03] E. Jeckelmann. Optical excitations in a one-dimensional Mott insulator. Physical Review B 67, 075106 (2003).

URL http://dx.doi.org/10.1103/PhysRevB.67.075106 
[JEK08] A. J. A. James, F. H. L. Essler, and R. M. Konik. Finite-temperature dynamical structure factor of alternating Heisenberg chains. Physical Review B 78, 094411 (2008). URL http://dx.doi.org/10.1103/PhysRevB.78.094411

[JG96] M. Jarrell and J. E. Gubernatis. Bayesian inference and the analytic continuation of imaginary-time quantum Monte Carlo data. Physics Reports 269(3), 133 (1996). URL http://dx.doi.org/10.1016/0370-1573(95)00074-7

[JGE00] E. Jeckelmann, F. Gebhard, and F. H. L. Essler. Optical Conductivity of the Half-Filled Hubbard Chain. Physical Review Letters 85, 3910 (2000). URL http://dx.doi.org/10.1103/PhysRevLett.85.3910

[Joh97] D. C. Johnston. Handbook of Magnetic Materials, volume 10. Elsevier Science, Netherlands (1997). Revise.

[JOK $\left.{ }^{+} 11\right] \quad$ H. Jeschke, I. Opahle, H. Kandpal, R. Valentí, H. Das, T. Saha-Dasgupta, O. Janson, H. Rosner, A. Brühl, B. Wolf, M. Lang, J. Richter, S. Hu, X. Wang, R. Peters, T. Pruschke, and A. Honecker. Multistep Approach to Microscopic Models for Frustrated Quantum Magnets: The Case of the Natural Mineral Azurite. Physical Review Letters 106, 217201 (2011).

URL http://dx.doi.org/10.1103/PhysRevLett.106.217201

[JP00] J. Jaklič and P. Prelovšek. Finite-temperature properties of doped antiferromagnets. Advances in Physics 49(1), 1 (2000).

URL http://dx.doi.org/10.1080/000187300243381

$\left[\mathrm{JQCI}^{+} 14\right] \quad$ J. Jensen, D. L. Quintero-Castro, A. T. M. N. Islam, K. C. Rule, M. Månsson, and B. Lake. Lineshape of the singlet-triplet excitations in the dimer system $\mathrm{Sr}_{3} \mathrm{Cr}_{2} \mathrm{O}_{8}$ to first order in the high-density 1/z expansion. Physical Review B 89, 134407 (2014).

URL http://dx.doi.org/10.1103/PhysRevB.89.134407

[Kan59] J. Kanamori. Superexchange interaction and symmetry properties of electron orbitals. Journal of Physics and Chemistry of Solids 10(2-3), 87 (1959).

URL http://dx.doi.org/10.1016/0022-3697(59)90061-7

[Kan63] J. Kanamori. Electron Correlation and Ferromagnetism of Transition Metals. Progress of Theoretical Physics 30(3), 275 (1963).

URL http://dx.doi.org/10.1143/PTP.30.275

$\left[\mathrm{KBC}^{+} 05\right] \quad$ M. Kenzelmann, C. D. Batista, Y. Chen, C. Broholm, D. H. Reich, S. Park, and Y. Qiu. $S=\frac{1}{2}$ chain in a staggered field: High-energy bound-spinon state and the effects of a discrete lattice. Physical Review B 71, 094411 (2005).

URL http://dx.doi.org/10.1103/PhysRevB.71.094411

[KBM12] C. Karrasch, J. H. Bardarson, and J. E. Moore. Finite-Temperature Dynamical Density Matrix Renormalization Group and the Drude Weight of Spin-1/2 Chains. Physical Review Letters 108, 227206 (2012).

URL http://dx.doi.org/10.1103/PhysRevLett.108.227206

[KBM13] C. Karrasch, J. H. Bardarson, and J. E. Moore. Reducing the numerical effort of finitetemperature density matrix renormalization group calculations. New Journal of Physics 15(8), 083031 (2013).

URL http://dx.doi.org/10.1088/1367-2630/15/8/083031

$\left[\mathrm{KCB}^{+} 04\right] \quad$ M. Kenzelmann, Y. Chen, C. Broholm, D. H. Reich, and Y. Qiu. Bound Spinons in an Antiferromagnetic $S=1 / 2$ Chain with a Staggered Field. Physical Review Letters 93, 017204 (2004).

URL http://dx.doi.org/10.1103/PhysRevLett.93.017204

[KE09] I. Kuzmenko and F. H. L. Essler. Dynamical correlations of the spin- $\frac{1}{2}$ Heisenberg XXZ chain in a staggered field. Physical Review B 79, 024402 (2009).

URL http://dx.doi.org/10.1103/PhysRevB.79.024402

[KHM98] M. Karbach, K. Hu, and G. Müller. Introduction to the Bethe Ansatz II. Computers in Physics 12(6), 565 (1998).

URL http://dx.doi.org/10.1063/1.168740 
$\left[\mathrm{KIM}^{+} 01\right] \quad$ M. Kohgi, K. Iwasa, J.-M. Mignot, B. Fåk, P. Gegenwart, M. Lang, A. Ochiai, H. Aoki, and T. Suzuki. Staggered Field Effect on the One-Dimensional $S=\frac{1}{2}$ Antiferromagnet $\mathrm{Yb}_{4} \mathrm{As}_{3}$. Physical Review Letters 86, 2439 (2001).

URL http://dx.doi.org/10.1103/PhysRevLett.86.2439

[KJ00] A. Klümper and D. C. Johnston. Thermodynamics of the Spin- 1/2 Antiferromagnetic Uniform Heisenberg Chain. Physical Review Letters 84, 4701 (2000).

URL http://dx.doi.org/10.1103/PhysRevLett.84.4701

[KKHM15] C. Karrasch, D. M. Kennes, and F. Heidrich-Meisner. Spin and thermal conductivity of quantum spin chains and ladders. Physical Review B 91, 115130 (2015).

URL http://dx.doi.org/10.1103/PhysRevB.91.115130

[KKM14] C. Karrasch, D. M. Kennes, and J. E. Moore. Transport properties of the one-dimensional Hubbard model at finite temperature. Physical Review B 90, 155104 (2014).

URL http://dx.doi.org/10.1103/PhysRevB.90.155104

[Klü98] A. Klümper. The spin-1/2 Heisenberg chain: thermodynamics, quantum criticality and spin-Peierls exponents. The European Physical Journal B 5(3), 677 (1998).

URL http://dx.doi.org/10.1007/s100510050491

[Kly16] E. S. Klyushina. Unconventional magnetic properties of the low dimensional quantum magnets $\mathrm{BaNi}_{2} \mathrm{~V}_{2} \mathrm{O}_{8}$ and $\mathrm{BaCu}_{2} \mathrm{~V}_{2} \mathrm{O}_{8}$ (in preparation). Ph.D. thesis, Technische Universität Berlin (2016).

$\left[\mathrm{KMB}^{+} 97\right] \quad$ M. Karbach, G. Müller, A. H. Bougourzi, A. Fledderjohann, and K.-H. Mütter. Twospinon dynamic structure factor of the one-dimensional $s=\frac{1}{2}$ Heisenberg antiferromagnet. Physical Review B 55, 12510 (1997).

URL http://dx.doi.org/10.1103/PhysRevB.55.12510

[KMGT97] M. Karbach, G. Müller, H. Gould, and J. Tobochnik. Introduction to the Bethe Ansatz I. Computers in Physics 11(1), 36 (1997).

URL http://dx.doi.org/10.1063/1.4822511

[Kop90] P. Kopietz. Magnon damping in the two-dimensional quantum Heisenberg antiferromagnet at short wavelengths. Physical Review B 41, 9228 (1990).

URL http://dx.doi.org/10.1103/PhysRevB.41.9228

[Kor79] V. Korepin. Direct calculation of the $S$ matrix in the massive Thirring model. Theoretical and Mathematical Physics 41(2), 953 (1979).

URL http://dx.doi.org/10.1007/BF01028501

[KPcv09] J. Kokalj and P. Prelovšek. Finite-temperature dynamics with the density-matrix renormalization group method. Physical Review B 80, 205117 (2009).

URL http://dx.doi.org/10.1103/PhysRevB.80.205117

[Kra34] H. Kramers. L'interaction Entre les Atomes Magnétogènes dans un Cristal Paramagnétique. Physica 1(1), $182(1934)$.

URL http://dx.doi.org/10.1016/S0031-8914(34)90023-9

$\left[\mathrm{KTF}^{+} 16\right] \quad$ E. S. Klyushina, A. C. Tiegel, B. Fauseweh, A. T. M. N. Islam, J. T. Park, B. Klemke, A. Honecker, G. S. Uhrig, S. R. Manmana, and B. Lake. Magnetic excitations in the $S=\frac{1}{2}$ antiferromagnetic-ferromagnetic chain compound $\mathrm{BaCu}_{2} \mathrm{~V}_{2} \mathrm{O}_{8}$ at zero and finite temperature. Physical Review B 93, 241109(R) (2016).

URL http://dx.doi.org/10.1103/PhysRevB.93.241109

[KU00] C. Knetter and G. Uhrig. Perturbation theory by flow equations: dimerized and frustrated $S=1 / 2$ chain. The European Physical Journal B 13(2), 209 (2000).

URL http://dx.doi.org/10.1007/s100510050026

[Kub57] R. Kubo. Statistical-Mechanical Theory of Irreversible Processes. I. General Theory and Simple Applications to Magnetic and Conduction Problems. Journal of the Physical Society of Japan 12(6), 570 (1957).

URL http://dx.doi.org/10.1143/JPSJ.12.570

[KW99] T. D. Kühner and S. R. White. Dynamical correlation functions using the density matrix renormalization group. Physical Review B 60, 335 (1999).

URL http://dx.doi.org/10.1103/PhysRevB.60.335 
[KW06] H.-J. Koo and M.-H. Whangbo. Importance of the $O-M-O$ Bridges $\left(M=V^{5+}\right.$, $\mathrm{Mo}^{6+}$ ) for the Spin-Exchange Interactions in the Magnetic Oxides of $\mathrm{Cu}^{2+}$ Ions Bridged by $\mathrm{MO}_{4}$ Tetrahedra: Spin-Lattice Models of $\mathrm{Rb}_{2} \mathrm{Cu}_{2}\left(\mathrm{MoO}_{4}\right)_{3}, \mathrm{BaCu}_{2} \mathrm{~V}_{2} \mathrm{O}_{8}$, and $\mathrm{KBa}_{3} \mathrm{Ca}_{4} \mathrm{Cu}_{3} \mathrm{~V}_{7} \mathrm{O}_{2} 8$. Inorganic Chemistry 45(11), 4440 (2006).

URL http://dx.doi.org/10.1021/ic060392w

[Lan50] C. Lanczos. An iteration method for the solution of the eigenvalue problem of linear differential and integral operators. Journal of Research of the National Bureau of Standards 45(4), 225 (1950).

URL http://dx.doi.org/10.6028/jres.045.026

[LCW06] J. Lou, C. Chen, and X. Wang. Field-induced midgap edge excitations in quantum spin chains. Physical Review B 73, 092407 (2006).

URL http://dx.doi.org/10.1103/PhysRevB.73.092407

$\left[\mathrm{LCZ}^{+} 05\right] \quad$ J. Lou, C. Chen, J. Zhao, X. Wang, T. Xiang, Z. Su, and L. Yu. Midgap States in Antiferromagnetic Heisenberg Chains with a Staggered Field. Physical Review Letters 94, 217207 (2005).

URL http://dx.doi.org/10.1103/PhysRevLett.94.217207

[LEK72] P. W. Langhoff, S. T. Epstein, and M. Karplus. Aspects of Time-Dependent Perturbation Theory. Reviews of Modern Physics 44, 602 (1972).

URL http://dx.doi.org/10.1103/RevModPhys.44.602

[LGG03] P. Lemmens, G. Güntherodt, and C. Gros. Magnetic light scattering in low-dimensional quantum spin systems. Physics Reports 375(1), 1 (2003).

URL http://dx.doi.org/10.1016/S0370-1573(02)00321-6

[LOV15] C. Lubich, I. V. Oseledets, and B. Vandereycken. Time Integration of Tensor Trains. SIAM Journal on Numerical Analysis 53(2), 917 (2015).

URL http://dx.doi.org/10.1137/140976546

[LRSV13] C. Lubich, T. Rohwedder, R. Schneider, and B. Vandereycken. Dynamical Approximation by Hierarchical Tucker and Tensor-Train Tensors. SIAM Journal on Matrix Analysis and Applications 34(2), 470 (2013).

URL http://dx.doi.org/10.1137/120885723

[LSM61] E. Lieb, T. Schultz, and D. Mattis. Two soluble models of an antiferromagnetic chain. Annals of Physics 16(3), 407 (1961).

URL http://dx.doi.org/10.1016/0003-4916(61)90115-4

[LTFN05] B. Lake, D. A. Tennant, C. D. Frost, and S. E. Nagler. Quantum criticality and universal scaling of a quantum antiferromagnet. Nature Materials 4, 329 (2005).

URL http://dx.doi.org/10.1038/nmat1327

[Luc08] C. Luckmann. Finite Temperature Dynamics of One-Dimensional Quantum Magnets. Ph.D. thesis, Leibniz Universität Hannover (2008).

URL http://d-nb.info/989831485

[Lut76] A. Luther. Eigenvalue spectrum of interacting massive fermions in one dimension. Physical Review B 14, 2153 (1976).

URL http://dx.doi.org/10.1103/PhysRevB.14.2153

[LW68] E. H. Lieb and F. Y. Wu. Absence of Mott Transition in an Exact Solution of the ShortRange, One-Band Model in One Dimension. Physical Review Letters 20, 1445 (1968).

URL http://dx.doi.org/10.1103/PhysRevLett.20.1445

[LX05] C. S. Lue and B. X. Xie. NMR investigation of $\mathrm{BaCu}_{2} \mathrm{~V}_{2} \mathrm{O}_{8}$ in alternating-chain and dimer-chain models. Physical Review B 72, 052409 (2005).

URL http://dx.doi.org/10.1103/PhysRevB.72.052409

[Mah00] G. D. Mahan. Many-Particle Physics. Kluwer Academic/Plenum Publishers, New York, 3rd edition (2000).

[Mak75] J. Makhoul. Linear Prediction: A Tutorial Review. Proceedings of the IEEE 63, 561 (1975).

URL http://dx.doi.org/10.1109/PROC.1975.9792 
[Mat77] M. Matsuura. Susceptibilities of $s=1 / 2$ Crystallographic Two Sublattice System. Journal of the Physical Society of Japan 43(5), 1805 (1977).

URL http://dx.doi.org/10.1143/JPSJ.43.1805

$\left[\mathrm{MBC}^{+} 08\right] \quad$ C. F. Macrae, I. J. Bruno, J. A. Chisholm, P. R. Edgington, P. McCabe, E. Pidcock, L. Rodriguez-Monge, R. Taylor, J. van de Streek, and P. A. Wood. Mercury CSD 2.0 - new features for the visualization and investigation of crystal structures. J. App. Crystallogr. 41(2), 466 (2008).

URL http://dx.doi.org/10.1107/S0021889807067908

[McC07] I. P. McCulloch. From density-matrix renormalization group to matrix product states. Journal of Statistical Mechanics: Theory and Experiment 2007(10), P10014 (2007).

URL http://dx.doi.org/10.1088/1742-5468/2007/10/P10014

[MK04] H.-J. Mikeska and A. K. Kolezhuk. Quantum Magnetism, volume 645 of Lecture Notes in Physics, chapter One-dimensional magnetism, pages 1-83. Springer, Berlin, Heidelberg (2004).

URL http://dx.doi.org/10.1007/BFb0119591

[ML71] W. Marshall and S. W. Lovesey. Theory of Thermal Neutron Scattering. Oxford University Press, Oxford (1971).

[ML06] H. J. Mikeska and C. Luckmann. Finite-temperature dynamics of the spin- $\frac{1}{2}$ bond alternating Heisenberg antiferromagnetic chain. Physical Review B 73, 184426 (2006).

URL http://dx.doi.org/10.1103/PhysRevB.73.184426

[MMN05] S. R. Manmana, A. Muramatsu, and R. M. Noack. Time evolution of one-dimensional Quantum Many Body Systems. AIP Conference Proceedings 789, 269 (2005).

URL http://dx.doi.org/10.1063/1.2080353

[Mor60a] T. Moriya. Anisotropic Superexchange Interaction and Weak Ferromagnetism. Physical Review 120, 91 (1960).

URL http://dx.doi.org/10.1103/PhysRev.120.91

[Mor60b] T. Moriya. New Mechanism of Anisotropic Superexchange Interaction. Physical Review Letters 4, 228 (1960).

URL http://dx.doi.org/10.1103/PhysRevLett.4.228

[Mor70] B. Morosin. The crystal structure of $\mathrm{Cu}\left(\mathrm{NO}_{3}\right)_{2} \cdot 2.5 \mathrm{H}_{2} \mathrm{O}$. Acta Crystallographica Section B 26(9), 1203 (1970).

URL http://dx.doi.org/10.1107/S0567740870003898

[MTBB81] G. Müller, H. Thomas, H. Beck, and J. C. Bonner. Quantum spin dynamics of the antiferromagnetic linear chain in zero and nonzero magnetic field. Physical Review B 24, 1429 (1981).

URL http://dx.doi.org/10.1103/PhysRevB.24.1429

[Muk95] S. Mukamel. Principles of Nonlinear Optical Spectroscopy. Oxford University Press, Oxford (1995).

[MVL03] C. Moler and C. Van Loan. Nineteen Dubious Ways to Compute the Exponential of a Matrix, Twenty-Five Years Later. SIAM Review 45(1), 3 (2003).

URL http://dx.doi.org/10.1137/S00361445024180

[NA16] A. Nocera and G. Alvarez. Symmetry-conserving purification of quantum states within the density matrix renormalization group. Physical Review B 93, 045137 (2016).

URL http://dx.doi.org/10.1103/PhysRevB.93.045137

[NC00] M. A. Nielsen and I. L. Chuang. Quantum Computation and Quantum Information. Cambridge University Press, Cambridge, UK (2000).

[Nis95] T. Nishino. Density Matrix Renormalization Group Method for 2D Classical Models. Journal of the Physical Society of Japan 64(10), 3598 (1995).

URL http://dx.doi.org/10.1143/JPSJ.64.3598 
[NM05] R. M. Noack and S. R. Manmana. Diagonalization- and Numerical Renormalization-GroupBased Methods for Interacting Quantum Systems. AIP Conference Proceedings 789, 93 (2005).

URL http://dx.doi.org/10.1063/1.2080349

[Nol14] W. Nolting. Grundkurs Theoretische Physik 6: Statistische Physik. Springer, Berlin, Heidelberg, 7th edition (2014).

URL http://dx.doi.org/10.1007/978-3-642-25393-5

[NWZvdL99] F. Naef, X. Wang, X. Zotos, and W. von der Linden. Autocorrelations from the transfermatrix density-matrix renormalization-group method. Physical Review B 60, 359 (1999). URL http://dx.doi.org/10.1103/PhysRevB.60.359

[OA97] M. Oshikawa and I. Affleck. Field-Induced Gap in $S=1 / 2$ Antiferromagnetic Chains. Physical Review Letters 79, 2883 (1997).

URL http://dx.doi.org/10.1103/PhysRevLett.79.2883

[OA02] M. Oshikawa and I. Affleck. Electron spin resonance in $S=\frac{1}{2}$ antiferromagnetic chains. Physical Review B 65, 134410 (2002).

URL http://dx.doi.org/10.1103/PhysRevB.65.134410

[OBS10] A. S. Ovchinnikov, I. G. Bostrem, and V. E. Sinitsyn. Cluster perturbation theory for spin Hamiltonians. Theoretical and Mathematical Physics 162(2), 179 (2010).

URL http://dx.doi.org/10.1007/s11232-010-0013-7

[OR95] S. Östlund and S. Rommer. Thermodynamic Limit of Density Matrix Renormalization. Physical Review Letters 75, 3537 (1995).

URL http://dx.doi.org/10.1103/PhysRevLett.75.3537

[PB13] P. Prelovšek and J. Bonča. Strongly Correlated Systems: Numerical Methods, chapter Ground State and Finite Temperature Lanczos Methods, pages 1-30. Springer, Berlin, Heidelberg (2013).

URL http://dx.doi.org/10.1007/978-3-642-35106-8_1

$\left[\mathrm{PBT}^{+} 15\right] \quad$ H. N. Phien, J. A. Bengua, H. D. Tuan, P. Corboz, and R. Orús. Infinite projected entangled pair states algorithm improved: Fast full update and gauge fixing. Physical Review B 92, 035142 (2015).

URL http://dx.doi.org/10.1103/PhysRevB.92.035142

[PC76] J. H. H. Perk and H. W. Capel. Antisymmetric exchange, canting and spiral structure. Physics Letters A 58(2), 115 (1976).

URL http://dx.doi.org/10.1016/0375-9601(76)90515-6

[PEAT14] I. Pižorn, V. Eisler, S. Andergassen, and M. Troyer. Real time evolution at finite temperatures with operator space matrix product states. New Journal of Physics 16(7), 073007 (2014).

URL http://dx.doi.org/10.1088/1367-2630/16/7/073007

[PEDC05] M. B. Plenio, J. Eisert, J. Dreißig, and M. Cramer. Entropy, Entanglement, and Area: Analytical Results for Harmonic Lattice Systems. Physical Review Letters 94, 060503 (2005).

URL http://dx.doi.org/10.1103/PhysRevLett.94.060503

[Pru14] T. Pruschke. Advanced Solid State Theory. Morgan \& Claypool Publishers, San Rafael (2014).

URL http://dx.doi.org/10.1088/978-1-627-05328-0

[PWKH99] I. Peschel, X. Wang, M. Kaulke, and K. Hallberg, editors. Density Matrix Renormalization A New Numerical Method in Physics. Springer, Berlin (1999).

[QCLI $\left.{ }^{+} 12\right] \quad$ D. L. Quintero-Castro, B. Lake, A. T. M. N. Islam, E. M. Wheeler, C. Balz, M. Månsson, K. C. Rule, S. Gvasaliya, and A. Zheludev. Asymmetric Thermal Line Shape Broadening in a Gapped 3D Antiferromagnet: Evidence for Strong Correlations at Finite Temperature. Physical Review Letters 109, 127206 (2012).

URL http://dx.doi.org/10.1103/PhysRevLett.109.127206

[Riv90] T. J. Rivlin. Chebyshev Polynomials: From Approximation Theory to Algebra and Number Theory. Wiley-Interscience, New York, 2nd edition (1990). 
$\left[\mathrm{RMC}^{+} 01\right] \quad$ H. M. Rønnow, D. F. McMorrow, R. Coldea, A. Harrison, I. D. Youngson, T. G. Perring, G. Aeppli, O. Syljuåsen, K. Lefmann, and C. Rischel. Spin Dynamics of the 2D Spin $\frac{1}{2}$ Quantum Antiferromagnet Copper Deuteroformate Tetradeuterate (CFTD). Physical Review Letters 87, 037202 (2001).

URL http://dx.doi.org/10.1103/PhysRevLett.87.037202

$\left[\right.$ RPK $\left.^{+} 96\right] \quad$ S. Ramasesha, S. K. Pati, H. R. Krishnamurthy, Z. Shuai, and J. L. Brédas. Symmetrized density-matrix renormalization-group method for excited states of Hubbard models. Physical Review B 54, 7598 (1996).

URL http://dx.doi.org/10.1103/PhysRevB.54.7598

[RZ06] A. Rapp and G. Zaránd. Dynamical correlations and quantum phase transition in the quantum Potts model. Physical Review B 74, 014433 (2006). URL http://dx.doi.org/10.1103/PhysRevB.74.014433

[Saa03] Y. Saad. Iterative Methods for Sparse Linear Systems. Society for Industrial and Applied Mathematics, 2nd edition (2003).

[Sac01] S. Sachdev. Quantum Phase Transitions. Cambridge University Press, Cambridge, UK (2001).

[San10] A. W. Sandvik. Computational Studies of Quantum Spin Systems. AIP Conference Proceedings 1297(1), 135 (2010).

URL http://dx.doi.org/10.1063/1.3518900

[Sch04] P. Schmitteckert. Nonequilibrium electron transport using the density matrix renormalization group method. Physical Review B 70, 121302(R) (2004).

URL http://dx.doi.org/10.1103/PhysRevB.70.121302

[Sch05a] U. Schollwöck. The density-matrix renormalization group. Reviews of Modern Physics 77, 259 (2005).

URL http://dx.doi.org/10.1103/RevModPhys.77.259

[Sch05b] U. Schollwöck. Time-dependent Density-Matrix Renormalization-Group Methods. Journal of the Physical Society of Japan 74(Suppl), 246 (2005).

URL http://dx.doi.org/10.1143/JPSJS.74S.246

[Sch11] U. Schollwöck. The density-matrix renormalization group in the age of matrix product states. Annals of Physics 326(1), 96 (2011).

URL http://dx.doi.org/10.1016/j.aop.2010.09.012

[SdV96] G. L. G. Sleijpen and H. A. V. der Vorst. A Jacobi-Davidson Iteration Method for Linear Eigenvalue Problems. SIAM Journal on Matrix Analysis and Applications 17(2), 401 (1996).

URL http://dx.doi.org/10.1137/S0895479894270427

[SJ07] Y. Singh and D. C. Johnston. Singlet ground state in the spin- $\frac{1}{2}$ dimer compound $\mathrm{Sr}_{3} \mathrm{Cr}_{2} \mathrm{O}_{8}$. Physical Review B 76, 012407 (2007).

URL http://dx.doi.org/10.1103/PhysRevB.76.012407

[SJ09] T. Shirakawa and E. Jeckelmann. Charge and spin Drude weight of the one-dimensional extended Hubbard model at quarter filling. Physical Review B 79, 195121 (2009).

URL http://dx.doi.org/10.1103/PhysRevB.79.195121

[SK02] J. Sirker and A. Klümper. Temperature-driven crossover phenomena in the correlation lengths of the one-dimensional $t$-J model. Europhysics Letters 60(2), 262 (2002).

URL http://dx.doi.org/10.1209/epl/i2002-00345-2

[SK05] J. Sirker and A. Klümper. Real-time dynamics at finite temperature by the density-matrix renormalization group: A path-integral approach. Physical Review B 71, 241101(R) (2005). URL http://dx.doi.org/10.1103/PhysRevB.71.241101

[SMD08] S. S. Salunke, A. V. Mahajan, and I. Dasgupta. Magnetic properties and electronic structure of $\mathrm{S}=1 / 2$ spin gap compound $\mathrm{BaCu}_{2} \mathrm{~V}_{2} \mathrm{O}_{8}$. Physical Review B 77, 012410 (2008). URL http://dx.doi.org/10.1103/PhysRevB.77.012410

[SN11] J. J. Sakurai and J. Napolitano. Modern quantum mechanics. Addison-Wesley, Pearson, Boston, 2nd edition (2011). 
[SPP02] D. Sénéchal, D. Perez, and D. Plouffe. Cluster perturbation theory for Hubbard models. Physical Review B 66, 075129 (2002).

URL http://dx.doi.org/10.1103/PhysRevB.66.075129

[SPPL00] D. Sénéchal, D. Perez, and M. Pioro-Ladrière. Spectral Weight of the Hubbard Model through Cluster Perturbation Theory. Physical Review Letters 84, 522 (2000).

URL http://dx.doi.org/10.1103/PhysRevLett.84.522

[SPV11] S. Singh, R. N. C. Pfeifer, and G. Vidal. Tensor network states and algorithms in the presence of a global U(1) symmetry. Physical Review B 83, 115125 (2011).

URL http://dx.doi.org/10.1103/PhysRevB.83.115125

$\left[\mathrm{SRB}^{+} 03\right] \quad$ M. B. Stone, D. H. Reich, C. Broholm, K. Lefmann, C. Rischel, C. P. Landee, and M. M. Turnbull. Extended Quantum Critical Phase in a Magnetized Spin- $\frac{1}{2}$ Antiferromagnetic Chain. Physical Review Letters 91, 037205 (2003).

URL http://dx.doi.org/10.1103/PhysRevLett.91.037205

[Sre93] M. Srednicki. Entropy and area. Physical Review Letters 71, 666 (1993).

URL http://dx.doi.org/10.1103/PhysRevLett.71.666

[SU03] K. P. Schmidt and G. S. Uhrig. Excitations in One-Dimensional $S=\frac{1}{2}$ Quantum Antiferromagnets. Physical Review Letters 90, 227204 (2003).

URL http://dx.doi.org/10.1103/PhysRevLett.90.227204

[Suz76] M. Suzuki. Generalized Trotter's formula and systematic approximants of exponential operators and inner derivations with applications to many-body problems. Communications in Mathematical Physics 51(2), 183 (1976).

URL http://dx.doi.org/10.1007/BF01609348

[Suz85] M. Suzuki. Thermo Field Dynamics in Equilibrium and Non-Equilibrium Interacting Quantum Systems. Journal of the Physical Society of Japan 54(12), 4483 (1985).

URL http://dx.doi.org/10.1143/JPSJ.54.4483

[SW10] E. M. Stoudenmire and S. R. White. Minimally entangled typical thermal state algorithms. New Journal of Physics 12(5), 055026 (2010).

URL http://dx.doi.org/10.1088/1367-2630/12/5/055026

[SY97] S. Sachdev and A. P. Young. Low Temperature Relaxational Dynamics of the Ising Chain in a Transverse Field. Physical Review Letters 78, 2220 (1997).

URL http://dx.doi.org/10.1103/PhysRevLett.78.2220

[SYK02] H. Sakurai, K. Yoshimura, and K. Kosuge. Isolated Dimers in an Alternating Chain of $\mathrm{BaCu}_{2} \mathrm{~V}_{2} \mathrm{O}_{8}$. Journal of the Physical Society of Japan 71(Suppl), 190 (2002).

URL http://dx.doi.org/10.1143/JPSJS.71S.190

$\left[\mathrm{TBR}^{+} 03\right] \quad$ D. A. Tennant, C. Broholm, D. H. Reich, S. E. Nagler, G. E. Granroth, T. Barnes, K. Damle, G. Xu, Y. Chen, and B. C. Sales. Neutron scattering study of two-magnon states in the quantum magnet copper nitrate. Physical Review B 67, 054414 (2003).

URL http://dx.doi.org/10.1103/PhysRevB.67.054414

[TCNT95] D. A. Tennant, R. A. Cowley, S. E. Nagler, and A. M. Tsvelik. Measurement of the spinexcitation continuum in one-dimensional $\mathrm{KCuF}_{3}$ using neutron scattering. Physical Review B 52, 13368 (1995).

URL http://dx.doi.org/10.1103/PhysRevB.52.13368

$\left[\mathrm{TDH}^{+} 13\right] \quad$ A. C. Tiegel, P. E. Dargel, K. A. Hallberg, H. Frahm, and T. Pruschke. Spin-spin correlations between two Kondo impurities coupled to an open Hubbard chain. Physical Review B 87, 075122 (2013).

URL http://dx.doi.org/10.1103/PhysRevB.87.075122

[TH90] S. Tyč and B. I. Halperin. Damping of spin waves in a two-dimensional Heisenberg antiferromagnet at low temperatures. Physical Review B 42, 2096 (1990).

URL http://dx.doi.org/10.1103/PhysRevB.42.2096

$\left[\mathrm{THP}^{+} 16\right] \quad$ A. C. Tiegel, A. Honecker, T. Pruschke, A. Ponomaryov, S. A. Zvyagin, R. Feyerherm, and S. R. Manmana. Dynamical properties of the sine-Gordon quantum spin magnet Cu-PM at zero and finite temperature. Physical Review B 93, 104411 (2016).

URL http://dx.doi.org/10.1103/PhysRevB.93.104411 
$\left[\right.$ TLJ $\left.^{+} 12\right] \quad$ D. A. Tennant, B. Lake, A. J. A. James, F. H. L. Essler, S. Notbohm, H.-J. Mikeska, J. Fielden, P. Kögerler, P. C. Canfield, and M. T. F. Telling. Anomalous dynamical line shapes in a quantum magnet at finite temperature. Physical Review B 85, 014402 (2012). URL http://dx.doi.org/10.1103/PhysRevB.85.014402

[TMPH14] A. C. Tiegel, S. R. Manmana, T. Pruschke, and A. Honecker. Matrix product state formulation of frequency-space dynamics at finite temperatures. Physical Review B 90, 060406(R) (2014).

URL http://dx.doi.org/10.1103/PhysRevB.90.060406

[TPCN93] D. A. Tennant, T. G. Perring, R. A. Cowley, and S. E. Nagler. Unbound spinons in the S $=1 / 2$ antiferromagnetic chain $\mathrm{KCuF}_{3}$. Physical Review Letters 70, 4003 (1993).

URL http://dx.doi.org/10.1103/PhysRevLett.70.4003

[Tro59] H. F. Trotter. On the Product of Semi-Groups of Operators. Proceedings of the American Mathematical Society 10(4), 545 (1959). URL http://dx.doi.org/10.2307/2033649

[Tsv07] A. M. Tsvelik. Quantum Field Theory in Condensed Matter Physics. Cambridge University Press, Cambridge, UK, 2nd edition (2007).

$\left[\mathrm{TVD}^{+} 16\right] \quad$ A. C. Tiegel, T. Veness, P. E. Dargel, A. Honecker, T. Pruschke, I. P. McCulloch, and F. H. L. Essler. Optical conductivity of the Hubbard chain away from half filling. Physical Review B 93, 125108 (2016).

URL http://dx.doi.org/10.1103/PhysRevB.93.125108

[UHT82] H. Umezawa, H.Matsumoto, and M. Tachiki. Thermo field dynamics and condensed states. North Holland, Amsterdam (1982).

[UTK $\left.{ }^{+} 15\right] \quad$ I. Umegaki, H. Tanaka, N. Kurita, T. Ono, M. Laver, C. Niedermayer, C. Rüegg, S. OhiraKawamura, K. Nakajima, and K. Kakurai. Spinon, soliton, and breather in the spin- $\frac{1}{2}$ antiferromagnetic chain compound $\mathrm{KCuGaF}_{6}$. Physical Review B 92, 174412 (2015). URL http://dx.doi.org/10.1103/PhysRevB.92.174412

$\left[\mathrm{UTO}^{+} 09\right] \quad$ I. Umegaki, H. Tanaka, T. Ono, H. Uekusa, and H. Nojiri. Elementary excitations of the $S=\frac{1}{2}$ one-dimensional antiferromagnet $\mathrm{KCuGaF}_{6}$ in a magnetic field and quantum sineGordon model. Physical Review B 79, 184401 (2009).

URL http://dx.doi.org/10.1103/PhysRevB.79.184401

$\left[\mathrm{UTO}^{+}{ }^{12}\right] \quad$ I. Umegaki, H. Tanaka, T. Ono, M. Oshikawa, and K. Sakai. Thermodynamic properties of

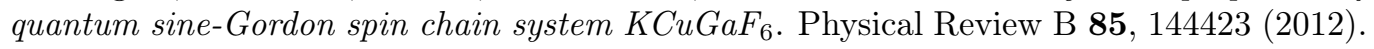
URL http://dx.doi.org/10.1103/PhysRevB.85.144423

[VC06] F. Verstraete and J. I. Cirac. Matrix product states represent ground states faithfully. Physical Review B 73, 094423 (2006).

URL http://dx.doi.org/10.1103/PhysRevB.73.094423

[VCM08] F. Verstraete, J. Cirac, and V. Murg. Matrix product states, projected entangled pair states, and variational renormalization group methods for quantum spin systems. Advances in Physics 57, 143 (2008).

URL http://dx.doi.org/10.1080/14789940801912366

[VGRC04] F. Verstraete, J. J. Garcia-Ripoll, and J. I. Cirac. Matrix Product Density Operators: Simulation of Finite-Temperature and Dissipative Systems. Physical Review Letters 93, 207204 (2004).

URL http://dx.doi.org/10.1103/PhysRevLett.93.207204

[Vid03] G. Vidal. Efficient Classical Simulation of Slightly Entangled Quantum Computations. Physical Review Letters 91, 147902 (2003).

URL http://dx.doi.org/10.1103/PhysRevLett.91.147902

[Vid04] G. Vidal. Efficient Simulation of One-Dimensional Quantum Many-Body Systems. Physical Review Letters 93, 040502 (2004). URL http://dx.doi.org/10.1103/PhysRevLett.93.040502

[Vil75] J. Villain. Propagative spin relaxation in the Ising-like antiferromagnetic linear chain. Physica B + C 79(1), 1 (1975).

URL http://dx.doi.org/10.1016/0378-4363(75)90101-1 
[VLRK03] G. Vidal, J. I. Latorre, E. Rico, and A. Kitaev. Entanglement in Quantum Critical Phenomena. Physical Review Letters 90, 227902 (2003).

URL http://dx.doi.org/10.1103/PhysRevLett.90.227902

[VMB90] R. Vogt and H. Müller-Buschbaum. BaCu $\mathrm{V}_{2} \mathrm{O}_{8}$ : Eine Variante des $\mathrm{SrNi}_{2} \mathrm{~V}_{2} \mathrm{O}_{8}$-Typs, mit $\mathrm{Cu}^{2+}$ in $4+1+1-K o o r d i n a t i o n . ~ Z e i t s c h r i f t$ für anorganische und allgemeine Chemie 591(1), 167 (1990).

URL http://dx.doi.org/10.1002/zaac.19905910119

[WA08] S. R. White and I. Affleck. Spectral function for the $S=1$ Heisenberg antiferromagetic chain. Physical Review B 77, 134437 (2008).

URL http://dx.doi.org/10.1103/PhysRevB.77.134437

[Wei12] A. Weichselbaum. Non-abelian symmetries in tensor networks: A quantum symmetry space approach. Annals of Physics 327(12), 2972 (2012).

URL http://dx.doi.org/10.1016/j.aop.2012.07.009

[WF04] S. R. White and A. E. Feiguin. Real-Time Evolution Using the Density Matrix Renormalization Group. Physical Review Letters 93, 076401 (2004).

URL http://dx.doi.org/10.1103/PhysRevLett.93.076401

[Whi92] S. R. White. Density matrix formulation for quantum renormalization groups. Physical Review Letters 69, 2863 (1992).

URL http://dx.doi.org/10.1103/PhysRevLett.69.2863

[Whi93] S. R. White. Density-matrix algorithms for quantum renormalization groups. Physical Review B 48, 10345 (1993).

URL http://dx.doi.org/10.1103/PhysRevB.48.10345

[Whi05] S. R. White. Density matrix renormalization group algorithms with a single center site. Physical Review B 72, 180403(R) (2005).

URL http://dx.doi.org/10.1103/PhysRevB.72.180403

[Whi09] S. R. White. Minimally Entangled Typical Quantum States at Finite Temperature. Physical Review Letters 102, 190601 (2009).

URL http://dx.doi.org/10.1103/PhysRevLett.102.190601

[WJMS15] F. A. Wolf, J. A. Justiniano, I. P. McCulloch, and U. Schollwöck. Spectral functions and time evolution from the Chebyshev recursion. Physical Review B 91, 115144 (2015).

URL http://dx.doi.org/10.1103/PhysRevB.91.115144

[WKD03] M.-H. Whangbo, H.-J. Koo, and D. Dai. Spin exchange interactions and magnetic structures of extended magnetic solids with localized spins: theoretical descriptions on formal, quantitative and qualitative levels. Journal of Solid State Chemistry 176(2), 417 (2003). URL http://dx.doi.org/10.1016/S0022-4596(03)00273-1

[WMPS14] F. A. Wolf, I. P. McCulloch, O. Parcollet, and U. Schollwöck. Chebyshev matrix product state impurity solver for dynamical mean-field theory. Physical Review B 90, 115124 (2014). URL http://dx.doi.org/10.1103/PhysRevB.90.115124

$\left[\mathrm{WRC}^{+} 03\right] \quad$ A. U. B. Wolter, H. Rakoto, M. Costes, A. Honecker, W. Brenig, A. Klümper, H.-H. Klauss, F. J. Litterst, R. Feyerherm, D. Jérome, and S. Süllow. High-field magnetization study of the $S=\frac{1}{2}$ antiferromagnetic Heisenberg chain $\left[\mathrm{PMCu}\left(\mathrm{NO}_{3}\right)_{2}\left(\mathrm{H}_{2} \mathrm{O}\right)_{2}\right]_{n}$ with a field-induced gap. Physical Review B 68, 220406(R) (2003).

URL http://dx.doi.org/10.1103/PhysRevB.68.220406

$\left[\mathrm{WVS}^{+}\right.$09] A. Weichselbaum, F. Verstraete, U. Schollwöck, J. I. Cirac, and J. von Delft. Variational matrix-product-state approach to quantum impurity models. Physical Review B 80, 165117 (2009).

URL http://dx.doi.org/10.1103/PhysRevB.80.165117

[WWAF06] A. Weiße, G. Wellein, A. Alvermann, and H. Fehske. The kernel polynomial method. Reviews of Modern Physics 78, 275 (2006).

URL http://dx.doi.org/10.1103/RevModPhys.78.275 
[WWS $\left.{ }^{+} 05\right] \quad$ A. U. B. Wolter, P. Wzietek, S. Süllow, F. J. Litterst, A. Honecker, W. Brenig, R. Feyerherm, and H.-H. Klauss. Giant Spin Canting in the $S=1 / 2$ Antiferromagnetic Chain $\left[\mathrm{CuPM}\left(\mathrm{NO}_{3}\right)_{2}\left(\mathrm{H}_{2} \mathrm{O}\right)_{2}\right]_{n}$ Observed by ${ }^{13} \mathrm{C}-N M R$. Physical Review Letters 94, 057204 (2005).

URL http://dx.doi.org/10.1103/PhysRevLett.94.057204

[WX97] X. Wang and T. Xiang. Transfer-matrix density-matrix renormalization-group theory for thermodynamics of one-dimensional quantum systems. Physical Review B 56, 5061 (1997). URL http://dx.doi.org/10.1103/PhysRevB.56.5061

$\left[\mathrm{YIA}^{+} 01\right] \quad$ M. Yasui, Y. Ishikawa, N. Akiyama, T. Ishida, T. Nogami, and F. Iwasaki. Dipyrimidinecopper(II) dinitrate complexes showing magnetic interactions. Acta Crystallographica Section B 57(3), 288 (2001).

URL http://dx.doi.org/10.1107/S0108768101002737

[Zam95] A. B. Zamolodchikov. Mass scale in the sine-Gordon model and its reductions. International Journal of Modern Physics A 10, 1125 (1995).

URL http://dx.doi.org/10.1142/S0217751X9500053X

$\left[Z C^{+} 11\right] \quad$ S. A. Zvyagin, E. Cižmár, M. Ozerov, J. Wosnitza, R. Feyerherm, S. R. Manmana, and F. Mila. Field-induced gap in a quantum spin- $\frac{1}{2}$ chain in a strong magnetic field. Physical Review B 83, 060409(R) (2011).

URL http://dx.doi.org/10.1103/PhysRevB.83.060409

[ZKKF04] S. A. Zvyagin, A. K. Kolezhuk, J. Krzystek, and R. Feyerherm. Excitation Hierarchy of the Quantum Sine-Gordon Spin Chain in a Strong Magnetic Field. Physical Review Letters 93, 027201 (2004).

URL http://dx.doi.org/10.1103/PhysRevLett.93.027201

[ZKKF05] S. A. Zvyagin, A. K. Kolezhuk, J. Krzystek, and R. Feyerherm. Electron Spin Resonance in Sine-Gordon Spin Chains in the Perturbative Spinon Regime. Physical Review Letters 95, 017207 (2005).

URL http://dx.doi.org/10.1103/PhysRevLett.95.017207

$\left[\mathrm{ZKvL}^{+}\right.$04] S. A. Zvyagin, J. Krzystek, P. H. M. van Loosdrecht, G. Dhalenne, and A. Revcolevschi. High-field ESR study of the dimerized-incommensurate phase transition in the spin-Peierls compound $\mathrm{CuGeO}_{3}$. Physica B: Condensed Matter 346-347, 1 (2004).

URL http://dx.doi.org/10.1016/j.physb.2004.01.009

[ZL05] I. A. Zaliznyak and S. H. Lee. Modern Techniques for Characterizing Magnetic Materials. Springer, Heidelberg (2005).

[ZMK $\left.{ }^{+} 15\right] \quad$ M. P. Zaletel, R. S. K. Mong, C. Karrasch, J. E. Moore, and F. Pollmann. Time-evolving a matrix product state with long-ranged interactions. Physical Review B 91, 165112 (2015). URL http://dx.doi.org/10.1103/PhysRevB.91.165112

[ZP73] H. J. Zeiger and G. W. Pratt. Magnetic Interactions in Solids. Clarendon Press, Oxford (1973).

[ZT15] I. A. Zaliznyak and J. M. Tranquada. Strongly Correlated Systems: Experimental Techniques, volume 180 of Springer Series in Solid-State Sciences, chapter Neutron Scattering and Its Application to Strongly Correlated Systems, pages 205-235. Springer, Berlin, Heidelberg (2015).

URL http://dx.doi.org/10.1007/978-3-662-44133-6_7

[ZV04] M. Zwolak and G. Vidal. Mixed-State Dynamics in One-Dimensional Quantum Lattice Systems: A Time-Dependent Superoperator Renormalization Algorithm. Physical Review Letters 93, 207205 (2004).

URL http://dx.doi.org/10.1103/PhysRevLett.93.207205

[Zvy12] S. A. Zvyagin. Spin dynamics of $S=1 / 2$ Heisenberg chains with a staggered transverse field: electron spin resonance studies (Review Article). Low Temperature Physics 38(9), 819 (2012).

URL http://dx.doi.org/10.1063/1.4752094 
$\left[Z_{W}{ }^{+} 06\right] \quad$ S. A. Zvyagin, J. Wosnitza, J. Krzystek, R. Stern, M. Jaime, Y. Sasago, and K. Uchinokura. Spin-triplet excitons in the $S=\frac{1}{2}$ gapped antiferromagnet $\mathrm{BaCuSi}_{2} \mathrm{O}_{6}$ : Electron paramagnetic resonance studies. Physical Review B 73, 094446 (2006).

URL http://dx.doi.org/10.1103/PhysRevB.73.094446

[ZWX $\left.{ }^{+} 03\right] \quad$ J. Z. Zhao, X. Q. Wang, T. Xiang, Z. B. Su, and L. Yu. Effects of the DzyaloshinskiiMoriya Interaction on Low-Energy Magnetic Excitations in Copper Benzoate. Physical Review Letters 90, 207204 (2003).

URL http://dx.doi.org/10.1103/PhysRevLett.90.207204

[ZZ79] A. B. Zamolodchikov and A. B. Zamolodchikov. Factorized S-matrices in two dimensions as the exact solutions of certain relativistic quantum field theory models. Annals of Physics 120(2), 253 (1979).

URL http://dx.doi.org/10.1016/0003-4916(79)90391-9 